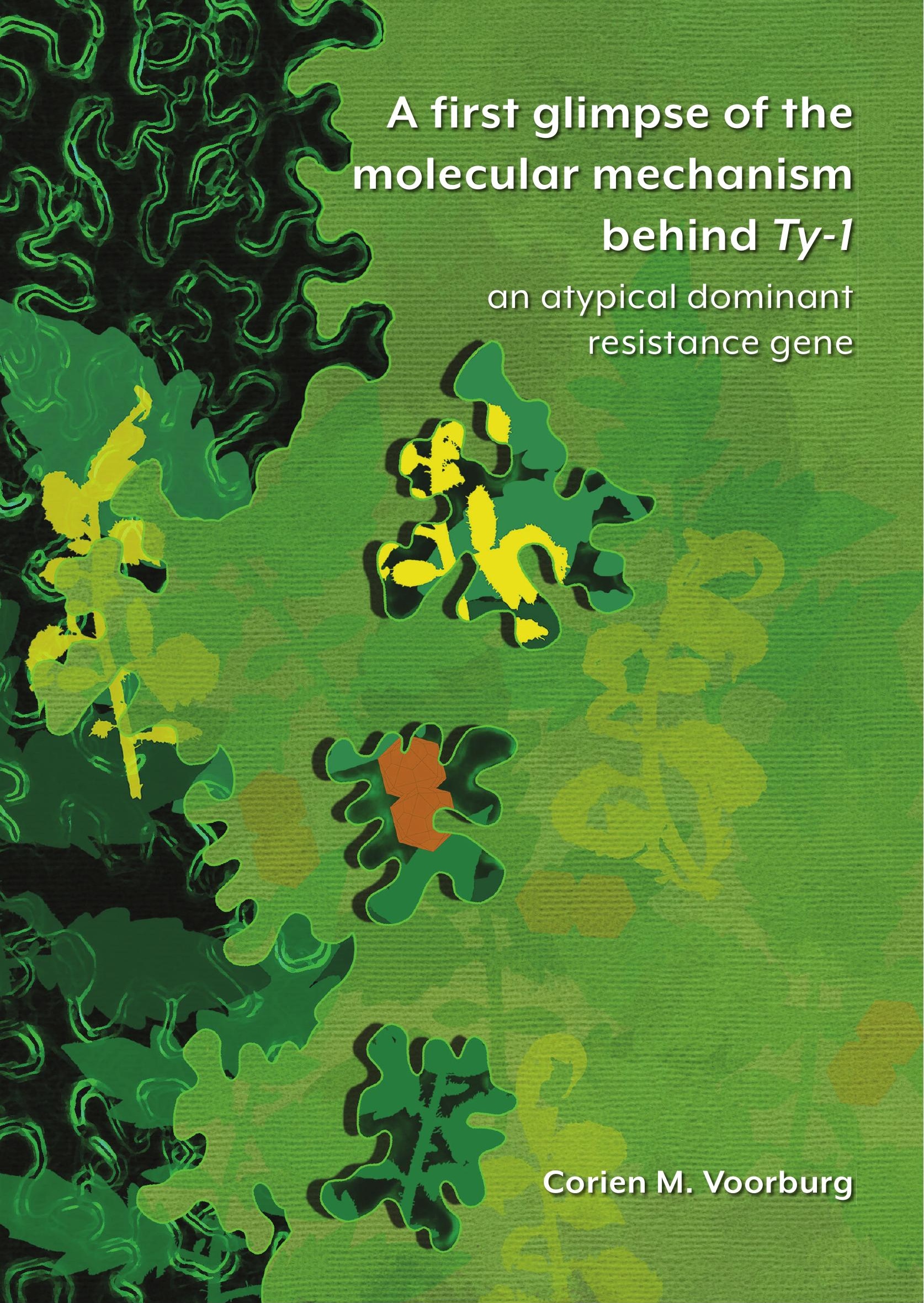




\section{Propositions}

1. The resistance gene Ty-1 confers resistance to all members of the Geminiviridae via enhancing the antiviral RNAi response. (this thesis)

2. Nuclear Cajal bodies are essential for the RNAi response against geminiviruses.

(this thesis)

3. The pressure to publish leads authors to ignore controls.

4. Team diversity in sense of gender, culture and field of expertise is the lifeblood of good research.

5. For nature preservation and biodiversity, the SARS-CoV-2 pandemic is a blessing in disguise.

6. If employers would provide people working from a home-office with a green-leaved plant, productivity, happiness and air quality will be boosted.

7. Dancing improves the quality of life.

Propositions belonging to the thesis entitled:

'A first glimpse of the molecular mechanism behind Ty-1, an atypical dominant resistance gene'

Corien M. Voorburg

Wageningen, 18 June 2021 


\section{A first glimpse of the molecular mechanism behind $T y-1$, an atypical dominant resistance gene}

Corien M. Voorburg 


\section{Thesis committee}

\section{Promotors}

Prof. Dr M.M. van Oers

Professor of Virology

Wageningen University \& Research

Dr R.J.M. Kormelink

Associate Professor, Laboratory of Virology

Wageningen University \& Research

Prof. Dr Y. Bai

Personal chair, Plant Breeding

Wageningen University \& Research

\section{Other members}

Prof. Dr G. Smant, Wageningen University \& Research

Dr P.M. Bleeker, University of Amsterdam

Dr K.M. Mäkinen, University of Helsinki, Finland

Prof. Dr E.R. Bejarano, University of Malaga, Spain

This research was conducted under the auspices of the Graduate School Experimental Plant Sciences 


\title{
A first glimpse of the molecular mechanism behind Ty-1, an atypical dominant resistance gene
}

\author{
Corien M. Voorburg
}

Thesis

submitted in fulfilment of the requirements for the degree of doctor at Wageningen University by the authority of the Rector Magnificus, Prof. Dr A.P.J. Mol, in the presence of the

Thesis Committee appointed by the Academic Board to be defended in public on Friday 18 June 2021 at 11 a.m. in the Aula. 
Corien M. Voorburg

A first glimpse of the molecular mechanism behind Ty-1, an atypical dominant resistance gene

220 pages.

PhD thesis, Wageningen University, Wageningen, the Netherlands (2021)

With references, with summary in English and Dutch

ISBN: 978-94-6395-808-0

DOI: https://doi.org/10.18174/546586 




\section{Table of Contents}

Chapter 1 General introduction 9

Chapter $2 \quad T y-1$, a universal resistance gene against geminiviruses that is compromised by co-replication of a betasatellite

Chapter 3 Small RNA profiling of susceptible and resistant Ty-1 encoding tomato plants upon TYLCV infection

Chapter 4 Analysis of cytoplasmic RNA Processing bodies in anti-viral 109 RNA interference of Tomato Yellow Leaf Curl geminivirus

Chapter 5 Ty-1 encoded RNA-dependent RNA polymerase from the gamma class, conferring resistance to geminiviruses, localizes to nuclear bodies involved in RNAi response and sumoylation

Chapter 6 General discussion

Appendices References 182

$\begin{array}{ll}\text { English summary } & 201\end{array}$

Nederlandse samenvatting $\quad 204$

Acknowledgements/Dankwoord 207

$\begin{array}{ll}\text { About the author } & 214\end{array}$

List of publications $\quad 215$

$\begin{array}{ll}\text { Education statement } & 216\end{array}$ 


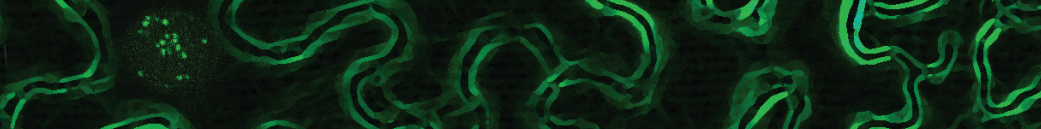

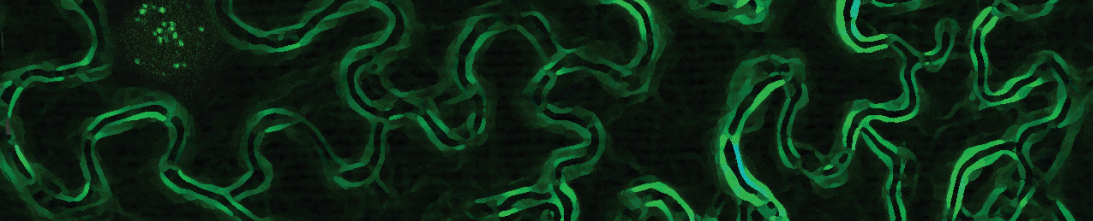
$\left\{\begin{array}{l}25 \\ 2\end{array}\right.$ Rुs

Af $\ln _{5}\left\{3 e^{2}\right.$

$\Leftrightarrow$

S.

)

) 5 .

रे (A)

5

St?

(i)

chn

(3)

$2 \pi$

री 2 है

(5) 05

$\frac{1}{2}$

Cov

?

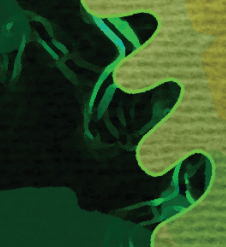

5) (untion 


\section{Chapter 1}

\section{General introduction}

C.M. Voorburg 


\section{TYLCV: a threat for tomato production}

Tomato Yellow Leaf Curl Disease (TYLCD) is a major threat for tomato production, especially in tropical and sub-tropical regions of the world. This disease was first observed in Israel in 1930, but it took more than 30 years before the etiology of the causing agent was identified and reported by Cohen et al.(Cohen and Harpaz, 1964). TYLCD is caused by several species of Tomato Yellow Leaf Curl Virus (TYLCV) belonging to the Begomovirus genus. The virus has spread over the world since 1930, now occurring in the Middle-east, Mediterranean area, North and Central America, Caribbean, East Asia, China and Australia (Mabvakure et al., 2016). One of the major factors that contributed to this spread is the appearance of the " $\mathrm{B}$ " biotype (also classified as the Middle East-Asia Minor 1 (MEAM1) species) of its vector the whitefly Bemisia tabaci (B. tabaci), with a broad host range, and the increase in vector populations (Cohen and Harpaz, 1964; Varma and Malathi, 2003). TYLCV causes crop losses in tomato production in both open field and greenhouses in many countries including Cyprus, Israel, Italy, Lebanon, Saudi Arabia and Spain. Yield losses can be up to $100 \%$. In the Dominican republic TYLCV was introduced in 1989 and there losses between 1989-1995 were estimated at US\$50 million (Czosnek, 2007; Morales and Anderson, 2001). The first symptoms of TYLCD show up in the apical leaves as slight yellowing of leaflet margins two to three weeks post infection. During later stages, the yellowing becomes more severe and leaves additionally start to curl and cup (Fig. 1.1A). Infected plants are often severely stunted with abscission of flowers and fruits (Friedmann et al., 1998; Lapidot, 2007; Lapidot and Friedmann, 2002).

In many countries with more temperate climate conditions B. tabaci "B" biotype and TYLCV are still absent and listed as quarantine agents. Preventing the introduction of the insect vector so far has been the most effective way to prevent the introduction of TYLCV. When the virus is already present, measurements to control TYLCV mainly focus on controlling the vector. Insecticides are used to decrease the whitefly populations while physical barriers, like nets, may prevent whiteflies from entering and feeding on the crops. However, the use of insecticides is not environmental friendly and whiteflies meanwhile have become resistant to many insecticides (Lapidot, 2007; Palumbo et al., 2001; Polston and Anderson, 1997). As a good 
A.

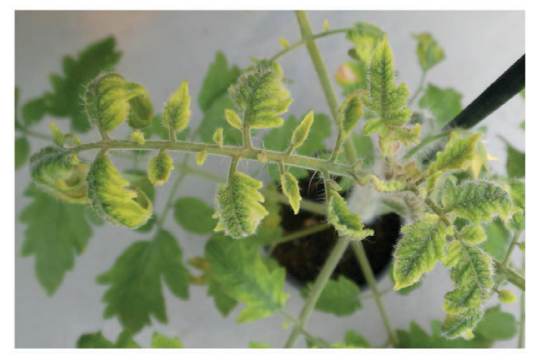

B.

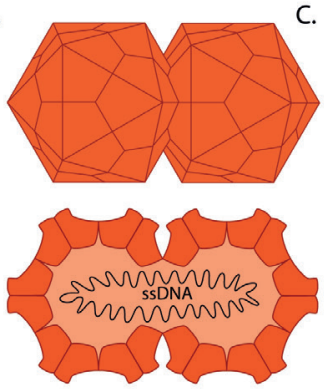

c.

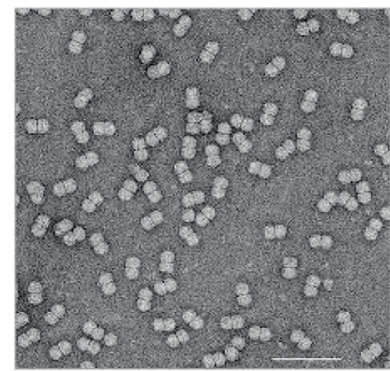

Figure 1.1: Symptoms caused by Tomato Yellow Leaf Curl Virus (TYLCV) infection and TYLCV particles.

A. Typical symptoms of TYLCV infection on susceptible tomato Moneymaker. Symptoms like yellowing and curling of the leaves are visible in the top of the tomato plant (own picture). B. Schematic overview of a geminivirus particle, consisting a single stranded circular DNA genome and the capsid protein. C. An Electron microscope image of purified begomovirus particles. Bar is $100 \mathrm{~nm}$ (Glick et al., 2009).

sustainable alternative to manage TYLCD, a lot of emphasis is focussed on breeding for TYLCV-resistance (Lapidot and Friedmann, 2002; Picó et al., 1996).

\section{Molecular biology and cytopathology of TYLCV}

TYLCV belongs to the Geminiviridae, a family whose members contain typical geminate (twin)-shaped particles with a circular single stranded (ss) DNA genome consisting of one or two elements (Fig. 1.1B,C). Members of this family are divided into nine genera named Becurtovirus, Begomovirus, Capulavirus, Curtovirus, Eragrovirus, Grablovirus, Mastrevirus, Topocuvirus and Turncurtovirus (Zerbini et al., 2017). The genera designation depends on host range, insect vector and genome organization (Table 1.1, Fig. 1.2). While most geminiviruses infect dicots, Mastreviruses infect monocots, are transmitted by leafhoppers and present the second largest group containing more than 30 species (Zerbini et al., 2017). The Begomovirus genus presents the largest group and contains more than 300 species, which are all transmitted by the whitefly $B$. tabaci, infect dicot plants and have a mono- or bipartite genome. Of those with a bipartite genome, the genetic elements are named DNA-A and DNA-B and generally 2.5-2.6 kilobases $(\mathrm{kb})$ in size. TYLCV is a monopartite begomovirus, and contains a circular ssDNA genome segment of 2.7-2.8 kb that encodes six genes (Fig. 1.2) (Zerbini et al., 2017). 
Table 1.1: The 9 genera within the geminivirus family and their characteristics.

\begin{tabular}{lllll} 
Genus & Vector & Host & Genome & No.Species \\
Begomovirus & white fly & dicot & $\begin{array}{l}\text { mono } \\
\text { and bi }\end{array}$ & $>350$ \\
\hline Topocuvirus & treehopper & dicot & mono & 1 \\
\hline Turncurtovirus & leafhopper & dicot & mono & 2 \\
\hline Capulavirus & $\begin{array}{l}\text { one species by aphid } \\
\text { other unknown }\end{array}$ & dicot & mono & 4 \\
Grablovirus & treehopper & dicot & mono & 1 \\
\hline Eragrovirus & unknown & monocot & mono & 1 \\
Curtovirus & leafhopper & dicot & mono & 3 \\
\hline Becurtovirus & leafhopper & dicot & mono & 2 \\
Mastrevirus & leafhopper & mono- and dicot & mono & $>30$
\end{tabular}

Monopartite begomoviruses often occur in nature together with one of two type of ssDNA satellite molecules of around $1.3 \mathrm{~kb}$, alphasatellites and betasatellites (Fig. 1.2). Alphasatellites encode a replication-initiator protein and therefore can self-replicate in plants. However, for movement and transmission by insects, alphasatellites depend on the associated begomovirus. The function of alphasatellites during begomovirus infections is not clear (Zhou, 2013). In contrary to alphasatellites, betasatellites are unable to self-replicate. They use the proteins of the associated begomovirus to replicate, but also to enable systemic spread throughout the plant and for insect transmission. Betasatellites are a pathogenicity determinant: upon co-infection of a begomovirus and betasatellite, symptoms turn more severe (Zhou, 2013). Betasatellites encode one protein named $\beta C 1$ and this protein has been shown to suppress the anti-viral RNA interference (RNAi) defence pathways in plants (see further below) (Li, Zhao, et al., 2017; Yang, Xie, et al., 2011; Zhou, 2013).

TYLCV, like most begomoviruses, is phloem limited and transmitted by $B$. tabaci in a persistent manner. Although the virus is generally believed to be transmitted in a circulative manner, there is still some debate on a possible replication within the whitefly host (Czosnek et al., 2017; Pakkianathan et al., 2015; Sánchez-Campos et al., 2016). 


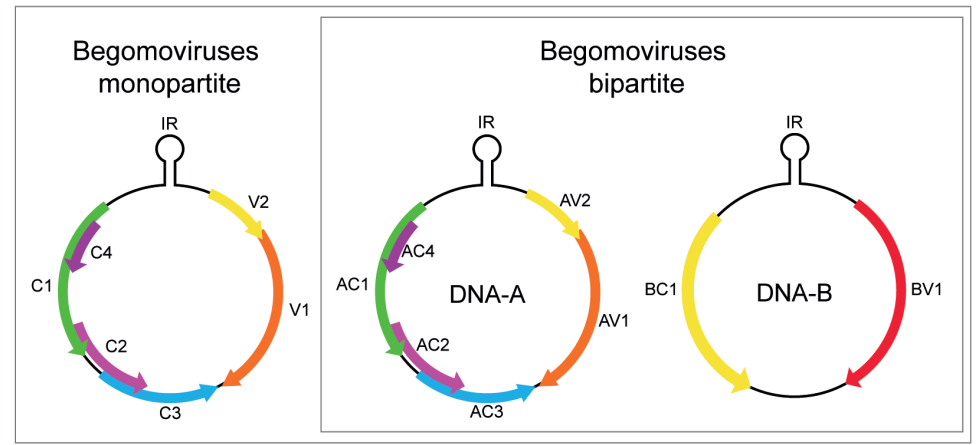

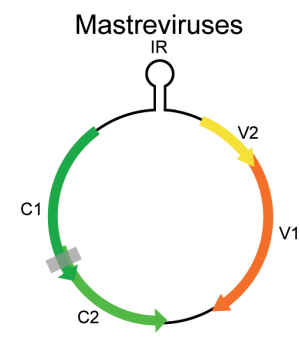

Alphasatellite

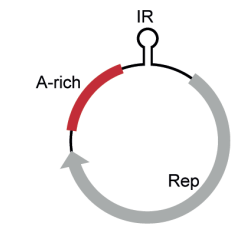

\begin{tabular}{|c|c|}
\hline rep & MP \\
\hline repA & ren
\end{tabular}

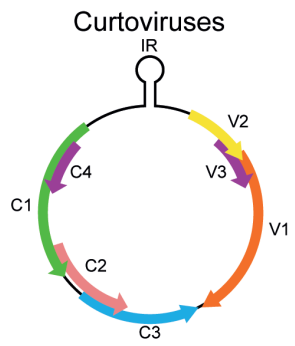

Betasatellite
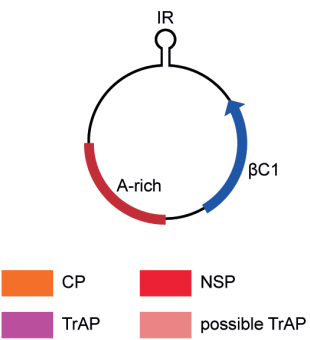

Figure 1.2: The genome structure of several geminiviruses.

The genome organisation of a monopartite and bipartite begomovirus, a mastrevirus and a curtovirus is shown. Also the genomes of apha- and betasatellites are depicted. The open reading frames on the virion sense (V) and complementary sense (C) are indicated with arrows, the adenine-rich region is depicted in red. An intron present in mastreviruses genes is indicated with a grey box. IR, Intergenic region (IR); Rep, replication-associated protein; $\mathrm{MP}$, movement protein; ren, replication enhancer protein; $\mathrm{CP}$, coat protein; TrAP, Transcriptional activator protein; NSP, nuclear shuttle protein.

After the virus enters the cell, genomic DNA is released and converted into double stranded (ds)DNA by plant DNA polymerases in the nucleus (Fig. 1.3) (Saunders et al., 1992). Next, these ds replicative intermediates bind to histone proteins and form mini chromosomes that act as templates for both transcription and 


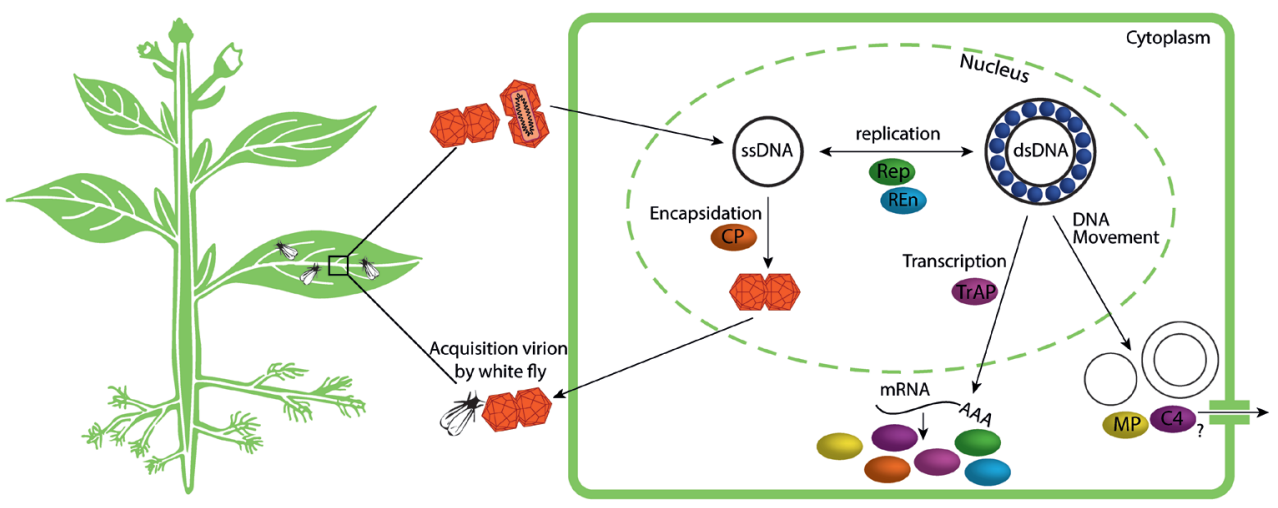

Figure 1.3: The life cycle of TYLCV.

After transmission of a virion by whitefies to the plant during feeding, the virion will enter the plant cell. Inside the cell ssDNA is released and converted into dsRNA which will form minichromosomes with host nucleosomes. After transcription by host RNA polymerase, Rep will initiate viral replication via Rolling Circle Replication or a recombination dependent mechanism. Replication is enhanced by REn. TrAP activates transcription and the produced messenger RNAs are translated by the host machinery. C4 and MP are involved in movement of the virus. The CP will encapsidate new ssDNA molecules, which can be acquired by feeding white flies.

replication (Pilartz and Jeske, 1992). The TYLCV genome is transcribed by host RNA polymerases and encodes six viral genes of which two are in the virion (V) sense strand, clockwise oriented, and named V1 and V2, while the other four genes are in the complementary (C) sense strand, anticlockwise, and named C1, C2, C3 and C4 (Fig. 1.2). The $V$ and $C$ sense genes are separated by an intergenic region (IR) at the genomic position around 12 o'clock. The IR sequence is variable, except for a highly conserved nanonucleotide sequence present within a hairpin structure (Padidam et al., 1995; Yang et al., 2014). The IR contains the bidirectional promoter of V1, V2, C1 and C4 and is essential for replication of the viral genome (Shivaprasad et al., 2005).

After transcription and translation of $\mathrm{C} 1$, also called Replication initiator protein (Rep), C1/Rep binds to the IR and forms a nick in the conserved nanonucleotide sequence, hereby initiating the Rolling Circle Replication (RCR) (Laufs et al., 1995; Stanley, 1995; Stenger et al., 1991). Next to RCR, geminiviruses also replicate via a recombination-dependent mechanism (Jeske et al., 2001). Newly formed ssDNA copies will be used either for replication or for virion formation. 
To favour the conditions for viral replication, cells are re-programmed by the virus. To this end $\mathrm{C} 1 /$ Rep binds to retinoblastoma-related protein ( $\mathrm{Rb})$, involved in cell cycle regulation, and alters the host cell to produce proteins involved in DNA replication (Arguello-Astorga et al., 2004; Gutierrez, 2000). The replication enhancer protein C3 (REn) is important in enhancing the replication. To this end, the protein interacts with $\mathrm{C} 1 /$ Rep and the host proteins proliferating-cell-nuclear-antigen (PCNA) and Rb, involved in DNA replication and cell cycle respectively (Castillo et al., 2003; Settlage et al., 2005; Sunter et al., 1990).

Next to C1/Rep and C3/REn, the capsid protein (V1/CP), a movement protein (V2/ $\mathrm{MP})$, a transcriptional activator protein (C2/TrAP) and a symptom determinant (C4) are expressed. C4 and V2/MP are suggested to be involved in cell-to-cell movement of viral DNA through plasmodesmata. Both genes are essential in tomato plants, since mutation of either C4 or V2 hampers systemic infection (Jupin et al., 1994; Priyadarshini et al., 2011; Rojas et al., 2001; Wartig et al., 1997). C2/TrAP activates the transcription of V1/CP (Sunter and Bisaro, 1991; Sunter and Bisaro, 1997). V1/CP shuttles viral DNA molecules from the nucleus to the cytoplasm and encapsidates ssDNA into virions, which can be acquired by whiteflies again (Rojas et al., 2001). $\mathrm{V} 1 / \mathrm{CP}$ is also necessary for systemic spread via sieve tubes (Noris et al., 1998) (Fig. 1.3).

\section{Mechanisms of defence against plant viruses}

Plants have developed various mechanisms to defend against plant pathogens. The most important ones are non-host resistance (NHR), expression of resistance $(R)$ genes, (alterations in) susceptibility $(S)$ genes and RNAi.

\section{Non-host resistance}

NHR applies if all genotypes of a plant species cannot be infected by a certain pathogen. The working mechanism of NHR has been studied more for bacterial and fungal infections, and less for viruses, but in general is still poorly understood (Gill 
et al., 2015; Lee et al., 2016). NHR comprises of multiple defence mechanisms and prevents pathogens to colonise the host plant. A first layer immediately encountered consists of physical barriers preventing pathogen invasion like trichomes, cell walls and/or secondary metabolite production. Since viruses overcome the cell wall barrier via mechanical inoculation or vector transmission, plant characteristics like high trichome density hampering insect feeding will help to reduce virus infection. A second layer of NHR is being induced by pattern recognition receptors (PRRs). PRRs recognize conserved structures associated with a group of pathogens, like flagellin for bacteria, and generally called pathogen associated molecular patterns (PAMPs). To date, all PRRs described in plants are membrane associated proteins that recognize extracellular PAMPs (Saijo et al., 2018). Recognition via PRRs induces a so-called PAMP-triggered immunity (PTI) response and results in necrosis at the site of entry (Gill et al., 2015). Mammals, besides the extracellular PRRs, also encode intracellular PRRs and some of these are able to sense viruses via ds RNA resulting from replicative intermediates. Although such PRRs have not been identified in plants yet, recent reports have shown that plant viruses do induce PTI signalling pathways most likely extracellularly and encode suppressors of this same pathway (Kørner et al., 2013; Nicaise and Candresse, 2017; Niehl et al., 2016). This suggests a role of PTI in viral infections in plants as well.

\section{Resistance- and Susceptibility-gene based resistance}

Another important layer of defence relies on $R$-genes. $R$ genes code for proteins that act as intracellular sensors of innate immunity. Several types of dominant $R$-genes are identified. Most $R$-genes completely abolish an infection, but some reduce replication (de Ronde et al., 2014). The class of $R$-genes that so far is most studied and used for resistance breeding against a diverse class of pathogens comprises the single dominant $R$-genes. Most of those belong to the NB-LRR type of $R$-genes i.e., they encode a protein that contains a central nucleotide binding (NB) domain and a c-terminal Leucine Rich Repeat (LRR) domain. The N-terminal end consists either of a coiled-coil (CC) or a Toll and Interleukin-1 receptor (TIR) domain (Moffett, 2009; de Ronde et al., 2014). For this reason NB-LRR proteins are also distinguished into CNL (CC-NB-LLR structure) or TNL (TIR-NB-LRR structure). Plant $R$-genes share structural 
homology with 2 classes of PRRs in animals, namely Nod-like receptors (NLR) and Toll-like receptors (TLR). Whereas NLRs share a NB and a LRR domain, TLRs share an intracellular TIR like domain and extracellular LRR domain (de Ronde et al., 2014). NB-LRR proteins generally sense a specific pathogen by (in)direct recognition of one of its proteins, called avirulence (Avr) determinant or effector. Upon recognition the conformation of the R-protein changes from an "off-state" into an "on-state" and consecutively triggers a signalling cascade that leads to a defence response. The molecular pathways of these defence responses still remain elusive (Kapos et al., 2019). $R$-gene mediated defence responses are mostly accompanied with a hypersensitive response (HR), caused by a programmed cell death, and visual by the formation of necrotic lesions on the leaf at the site of pathogen entry. An HR response is generally fast and occurs within 3 to 4 days post infection. Typical for this kind of $R$-gene response is that it mostly leads to an absolute resistance of the host i.e., infection and spread of the pathogen from the site of entry is prevented (de Ronde et al., 2014). The downside of using $R$-genes is that it also puts a selection pressure on pathogens. For many dominant NB-LRR $R$-genes resistance breaking strains have been found, and their Avr/effector protein shown to contain mutations that prevent (in)direct recognition by the R-protein (de Ronde et al., 2013). NB-LRR $R$-genes and the corresponding avr proteins are identified for fungi, nematodes, bacteria and viruses in many crops, as recently reviewed by Kapos et al. (2019).

Next to dominant resistance, recessive resistance is found against pathogens. Pathogens require host factors, so-called $S$-factors, to establish an infection. When such host factor is lost or altered (an allelic variant/isoform), pathogen replication is halted and this leads to $S$-gene based resistance. However, to obtain this resistance all gene copies generally need to be of this "altered" form and therefore this resistance is recessive (van Schie and Takken, 2014). A well-known S-gene is the mildew resistance locus O (MLO) gene. MLO is required for powdery mildew penetration of epidermal cells and loss of susceptibility is observed in mlo mutants. One of the best studied cases of recessive resistance against viruses involves elF4E and elF4G, two proteins that need to interact with the potyviral Viral protein genome-linked (VPg) to support viral replication. Isoforms of elF4E and elF4G exist that are incompatible with VPg. Plants containing these isoforms are not affected in their development due to some redundancy, but have lost their susceptibility 
to the virus (Combe et al., 2005; Gallois et al., 2010; Nicaise et al., 2007; Robaglia and Caranta, 2006; van Schie and Takken, 2014). Since S-genes may have a function in different processes, mutations can have pleiotropic effects (Pavan et al., 2010). Recessive resistance is thought to be more durable than breeding for dominant resistance genes.

\section{Antiviral RNAi and viral counter defence}

One of the main defence pathways viruses encounter is RNAi, also called RNA silencing. The first step in the RNAi pathway is the formation of dsRNA molecules. Double stranded RNA molecules are recognised by Dicer-like (DCL) proteins which have ribonuclease enzymatic activity and process the dsRNA into small interfering RNA (siRNA) molecules of 21-22 nucleotide (nt) in size. Next, the siRNA duplex molecule is unwound and one strand is incorporated into a RNA-induced silencing complex (RISC), from which Argonaute (AGO) is the core-component (Hammond, 2005). The RISC complex now has become activated and surveils for RNA molecules with sequence complementarity to the loaded siRNA strand, and targets these for either degradation or translational arrest (Fig. 1.4A) (Mallory and Vaucheret, 2010). This branch of the RNAi pathway acts in the cytoplasm and is named Post Transcriptional Gene Silencing (PTGS). Next to the PTGS response, a second RNAi pathway is important in plants. This pathway is also based on siRNAs, but of $24 \mathrm{nt}$ in size. RISC complexes loaded with a $24 \mathrm{nt}$ siRNA target cytosine methyltransferases to complementary DNA molecules, resulting in methylation of cytosines and in this way inhibiting the transcription (Fig. 1.4A,B) (Mallory and Vaucheret, 2010). This RNAi pathway is named Transcriptional Gene Silencing (TGS) and takes place in the nucleus.

In plants, but not insect and animals, the RNAi response is amplified by the action of host-encoded RNA-dependent RNA polymerases (RDRs) (Donaire et al., 2008; Wang et al., 2010). During this process, RDRs recognize aberrant RNA molecules, e.g. resulting from primary siRNA-mediated cleavage, and converts these into dsRNA with the help of cofactor SUPPRESSOR OF GENE SILENCING 3 (SGS3). This dsRNA is subsequently cleaved by DCLs into a population of secondary siRNAs 
A.

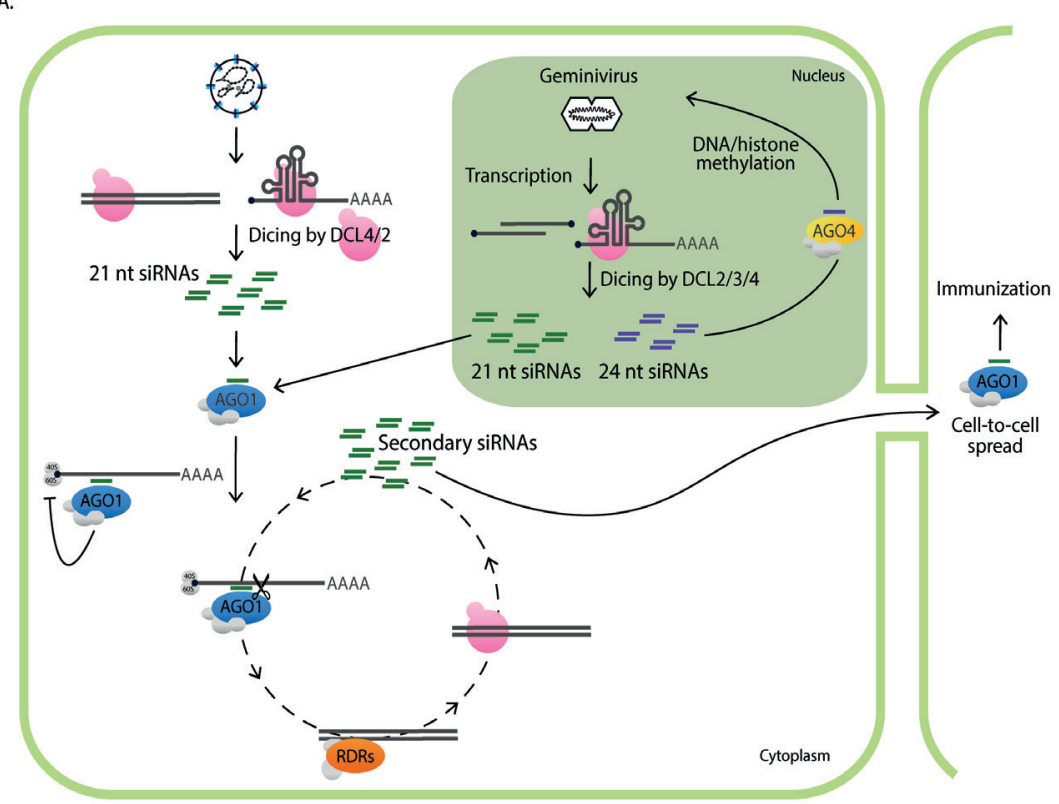

B.

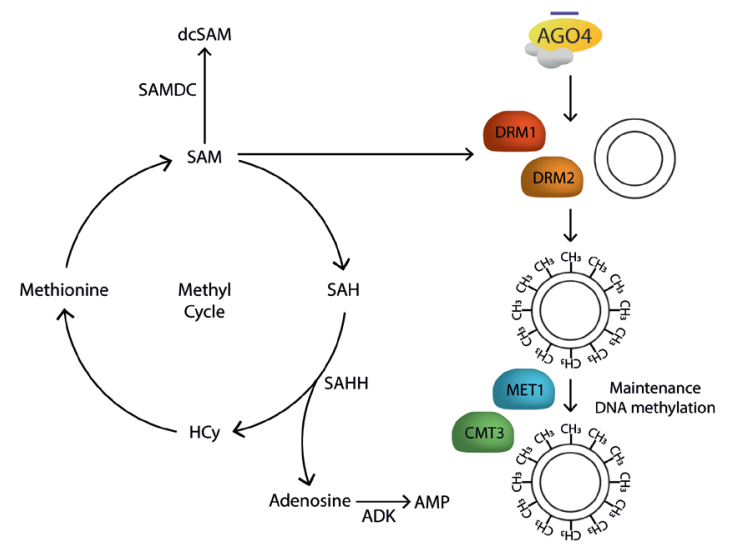

Figure 1.4: The antiviral RNAi response in plants.

A. The scheme depicts both the cytoplasmic PTGS and nuclear TGS pathway. When a RNA virus enters the cell, dsRNA (from replication intermediates or folding structures) is recognized and cleaved by DCL 4 or DCL2 in 21 nt siRNAs. The siRNA is unwound and uploaded in a AGO1 containing RISC complex which will target complementary RNA molecules for degradation or translational arrest. RDR6 will convert the aberrant RNA molecule into dsRNA, which will be cleaved by DCL into secondary siRNAs. When a geminivirus infects a plant cell, dsRNA will occur from folding structures or overlapping bidirectional transcripts. The dsRNA is cleaved by DCL3 into 24 nt siRNAs,

which are uploaded in a AGO4 containing RISC complex. The RISC complex will target complementary DNA sequences for methylation. B. The last step of the TGS response is depicted in more detail. When AGO4 targets the complementary viral DNA, it will recruit methyltransferases like DRM1, DRM2 and MET1. These transfer a methyl group from SAM to the cytosines in the viral DNA. SAM is produced via a methyl cycle in which several enzymes like SAHH, ADK and SAMDC play important roles and which are targeted by geminiviruses RSS proteins. 
(Fig. 1.4A). This amplification not only contributes to a strong (antiviral) RNAi response in plants, but also leads to the production of siRNAs outside the original target sequence, a process called transitive silencing (Mlotshwa et al., 2008). Production of secondary siRNAs is also essential for systemic silencing (Mermigka et al., 2016). Other differences in the RNAi pathway between mammals, insects and plants concern the number of proteins involved in the process: Plants encode four DCLs, where in mammals only one Dicer protein is found and in Drosophila, as model organism for insects, two Dicers (Rossi, 2005). Also the number of AGO proteins differ: four in mammals, two in drosophila and ten in arabidopsis (Höck and Meister, 2008; Wang et al., 2012). In plants, six RDRs are present while none is found in insects and animals (Willmann et al., 2011).

RNAi is a highly conserved mechanism in all eukaryotes with a major role in the regulation of gene expression, chromosome dynamics and control of transposable elements (TEs), but also is involved in the silencing of transgenes and repression of virus replication (Ding and Voinnet, 2007). In plants three major endogenous RNAi pathways are recognized that differ in function, in source of dsRNA and in biogenesis of small RNAs but share a similarity in action: 1 ) the microRNA (miRNA) pathway for regulation of gene expression, 2) the repeat-associated siRNA (rasiRNA) pathway for control of TEs, and 3) trans-acting siRNA (tasiRNA) precursor and aberrant RNA pathway (Pumplin and Voinnet, 2013). Each of them involves the action of different DCLs, RDRs and AGO proteins. MicroRNAs show resemblance to siRNAs in their size and structure but differ from siRNAs in that they arise from transcription of host (miRNA) genes encoding imperfectly matched hairpins. MiRNA precursors are processed in the nucleus by DCL1 and the co-factor protein HYPONASTIC LEACES 1 (HYL1) mostly into $21 \mathrm{nt}$-, and less into $22 \mathrm{nt}$-duplex molecules, of which one strand becomes loaded into an AGO1-containing RISC complex to mediate PTGS of host genes (Han et al., 2004; Zeng et al., 2003). The rasiRNA pathway is a nuclear pathway in which long dsRNA is produced by RDR2 from RNA polymerase IV transcripts of DNA repeats or transposon loci. Their processing by DCL3 generates $24 \mathrm{nt}$ rasiRNAs that load into an AGO4-containing RISC complex (sometimes containing AGO6 or AGO9) to mediate RNA-dependent DNA Methylation (RdDM) at rasiRNA loci (e.g. TEs) (Duan et al., 2015; Law and Jacobsen, 2010; Zheng et al., 2007). To this end, the activated RISC docks on nascent RNA polymerase $V$ transcripts of 
the rasiRNA loci and recruits methyl-transferases like DOMAINS REARRANGED METHYLTRANSFERASE (DRM)1 and DRM2, to transfer a methyl group from S-adenosyl methionine (SAM) to cytosine residues within the rasiRNA encoding genome sequence (Matzke et al., 2009; Matzke and Mosher, 2014). The substrate SAM is produced by the so-called methylation cycle where multiple enzymes play a role (Fig. 1.4B)(Mahfouz, 2010; Ulrey et al., 2005). tasiRNA precursors are singlestranded (non-coding) RNA molecules that become cleaved by a miRNA loaded AGO, after which RDR6 is recruited to the cleaved, aberrant RNA and converts it into dsRNA. Its subsequent processing by DCL4 generates $21 \mathrm{nt}$ tasiRNAs, to support PTGS of target transcripts that often belong to multi-gene families (Cuperus et al., 2010; Pumplin and Voinnet, 2013).

RNAi can also turn antiviral. During an infection with RNA viruses dsRNA arises from replicative intermediates or folding structures in genomic or messenger RNAs (mRNAs) (Ding and Voinnet, 2007). Upon cleavage into siRNAs and activation of RISC, viral (m)RNA molecules are targeted for degradation and in this way the production of viral proteins and genome replication is being inhibited. In order to successfully infect plants, viruses have evolved several strategies to evade or suppress the RNAi mechanism (Csorba et al., 2015). Evasion can take place via e.g. viral replication in membrane structures to prevent recognition by the cytoplasmic RNAi machinery (Schwartz et al., 2002). Another evasion strategy is to produce RNA from noncoding regions that function as decoy-RNA. By sequestering DCLs, viral coding transcripts are less prone to antiviral RNAi (Blevins et al., 2011; Schnettler et al., 2012). Next to evasion, suppression is the most commonly used strategy of plant viruses to overcome the RNAi machinery. Many plant viruses code for a RNA Silencing Suppressor (RSS) protein. RSS proteins are very diverse in structure and function and almost every main step in the RNAi pathway is targeted by a RSS protein (Pumplin and Voinnet, 2013). Many RSS proteins bind to long and/or short dsRNA. For example, the RSS NSs of Tomato Spotted Wilt Virus (TSWV) binds dsRNA size-independently and thereby interferes with the accessibility and processing of dsRNA by DCL into siRNAs, and, by binding to siRNAs, inhibits RISC activation and systemic spread (Hedil et al., 2015; Hedil et al., 2017; Schnettler et al., 2010). On the other hand, the RSS P19 of Carnation Italian Ringspot Virus (CIRV) binds selectively to siRNAs only (Vargason et al., 2003). Other RSS proteins have been 
shown to prevent RISC formation by binding to AGO proteins (e.g. $2 \mathrm{~b}$ of Cucumber Mosaic Virus (CMV)) or by targeting AGO for degradation (e.g. PO of Beet Western Yellows Virus (BWYV)) (Baumberger et al., 2007; Csorba et al., 2010; González et al., 2010; Hamera et al., 2012). The potexvirus triple-gene-block protein 1 (TGBp1) interacts with and aggregates SGS3 and RDR6, thereby interfering with the PTGS amplification cycle (Okano et al., 2014). Pns10 of Rice Dwarf Phytoreovirus (RDV) downregulates the expression of RDR6 (Csorba et al., 2015; Hedil and Kormelink, 2016; Ren et al., 2010). Plants compromised in the RNAi pathway, e.g. deficient in RDR1/RDR6, generally exhibit hyper-susceptibility to virus infection (Garcia-Ruiz et al., 2010; Jiang et al., 2012; Wang et al., 2010; Xie et al., 2001; Yang et al., 2004). Conversely, plant viruses from which the RSS has been made dysfunctional, show an attenuated disease phenotype (Azevedo et al., 2010; Garcia-Ruiz et al., 2010; Pumplin and Voinnet, 2013; Raja et al., 2008; Wang et al., 2011).

\section{RNAi and counter-defence by geminiviruses}

TYLCV, containing a ssDNA genome, is prone to both branches of the antiviral RNAi pathway i.e., PTGS and TGS. It is generally assumed that during an infection, secondary folding structures in mRNAs and/or overlapping transcripts resulting from bidirectional genome transcription are the source of dsRNA (Fig. 1.2, overlap c3/v1) (Ramesh et al., 2017). During a geminivirus infection 21, 22 and 24 nt antiviral siRNAs are produced and this indicates the activation of both the PTGS and TGS pathways (Aregger et al., 2012; Kuria et al., 2017; Piedra-Aguilera et al., 2019; Yang, Wang, et al., 2011). It is generally thought that the TGS pathway is playing a more important role in the anti-geminiviral defence than PTGS, since Arabidopsis thaliana knock out plants deficient in different steps of the methylation pathway are hypersusceptible to geminiviruses, while a RDR6 knock out does not show increased susceptibility (Jackel et al., 2016; Raja et al., 2008). Arabidopsis knock outs of polymerase IV, $\mathrm{V}, \mathrm{DCL3}, \mathrm{AGO4}$ and cytosine methyltransferases are hypersusceptible to the begomovirus Cabbage Leaf Curl Virus (CaLCuV) and/or curtovirus Beet curly top virus (BCTV) (Jackel et al., 2016; Raja et al., 2008). In contrast, a knock out of RDR2 Arabidopsis does not increase susceptibility to geminiviruses and suggests that RDR2, required for TGS against TEs, is not involved in the antiviral RNAi response 
against geminiviruses (Raja et al., 2008). Interestingly, antiviral siRNAs produced against the bipartite CaLCuV do not drastically change in RDR1,2,6 triple knockout plants compared to wild type plants (Aregger et al., 2012). Altogether, these studies indicate that the amplification of geminiviral siRNAs does not seem to depend on the involvement of RDR1,2 and 6 but instead might require other RDRs.

Whereas many (plant) viruses have been found to encode only one RSS protein, geminiviruses have evolved several RSS proteins (Table 1.2, at end of this chapter). Some of those suppress PTGS in various ways. The C2/TrAP protein stimulates transcription of host genes that inhibit PTGS responses (Trinks et al., 2005). V2/ MP of TYLCV interacts with SGS3, the important co-factor of RDR6, to inhibit the amplification of PTGS, while V2 of Cotton leaf curl Multan virus (CLCUMV) binds dsRNAs size-independently. As a result it interferes in the processing of long dsRNA by DCL into siRNAs, and their subsequent uploading into RISC and systemic spread (Amin et al., 2011; Glick et al., 2008; Rishishwar and Dasgupta, 2019; Zhang et al., 2012). C1/Rep of mastreviruses and C4 from cassava-infecting geminiviruses bind siRNAs (Chellappan et al., 2005; Y., Wang et al., 2014). The bipartite Mungbean yellow mosaic Indian virus encoded AC2 inhibits RDR6 and AGO1 (Kumar et al., 2015). $\beta C 1$ upregulates the expression of a Calmodulin like protein (CaM), which in turn represses the expression of RDR6 and targets SGS3, the co-factor of RDR6, for degradation via the autophagy pathway (Li et al., 2014). Geminiviral proteins have also been reported to suppress TGS, mostly by targeting different steps of the methylation pathway. C2/TrAP inhibits an adenosine kinase (ADK), an important enzyme in the methyl cycle, hindering in this way the methylation of cytosines (Buchmann et al., 2009; Wang et al., 2003) (Fig. 1.4B). C2/TrAP of Beet severe curly top virus (BSCTV) inhibits the degradation of S-adenosyl-methionine decarboxylase 1 (SAMDC) (Zhang et al., 2011), an inhibitor of the methyl cycle, while the $\beta C 1$ protein inhibits S-adenosyl homocysteine hydrolase (SAHH), another enzyme involved in the methyl cycle (Yang, Xie, et al., 2011) (Fig. 1.4B). C1/Rep together with C4 decreases the expression of two different methyltransferases (RodríguezNegrete et al., 2013). Methylation of cytosines often is associated with methylation of histones and results in transcriptional inactive chromatin, a process that is also suppressed by geminiviruses: C2/TrAP inhibits the histone methyltransferase 
Su(var)3-9 homolog 4/ Kryptonite (SUVH4/KYP), thereby inhibiting the formation of silenced chromatin (Castillo-González et al., 2015).

\section{Resistance against TYLCV}

To combat TYLCV, resistance breeding and resistance engineering are the most effective strategies that either partially or completely blocks virus replication. Examples for resistance engineering involve (1) expression of truncated $\mathrm{c} 1 /$ rep viral proteins in plants to interfere with the replication of the virus (Brunetti et al., 1997), (2) expression of non-plant proteins, like recombinant antibodies or the $B$. tabaci derived protein GroEL, to "trap" the virus and inhibit infection (Akad et al., 2007; Safarnejad et al., 2009), (3) clustered-regularly-interspaced-short-palindromicrepeats (CRISPRs)/CRISPR associated 9 (Cas9)-based cleavage of viral DNA sequences to inactivate genomic elements for replication (Ali et al., 2015), and (4) expression of viral sequences in an inverted-repeat format to induce RNAi-mediated resistance (Ammara et al., 2015; Leibman et al., 2015; Zrachya, Kumar, et al., 2007). Although these strategies have been shown to successfully reduce viral infection they receive societal reluctance, and deployment of resistance genes therefore still is the most commonly used approach.

While cultivated tomato, Solanum (S.) Lycopersicum, is susceptible to TYLCV infection, six resistance genes are currently available for introgression breeding, named $T y-1$ to -6 (Table 1.3). Most genes have been identified in wild tomato species S. chilense and S. habrochaites, while ty-5 (assumingly derived from S. peruvianum) is found in a domesticated tomato. Plants containing one of these genes do not show an HR upon infection and viral replication can still be detected in these plants, although the level of replication is much lower compared to the level in susceptible cultivars (Hanson et al., 2006; Hutton and Scott, 2014; Ji et al., 2007; Ji et al., 2009; Yan et al., 2018; Zamir et al., 1994). From the six resistance genes only Ty-4 and ty-6, both from $S$. chilense, have not been cloned yet and their mode of action remains unknown (Hutton and Scott, 2014; Ji et al., 2009). Ty-2, from S. habrochaites, has been cloned and encodes a NB-LRR protein (Yamaguchi et al., 2018). Resistance breaking strains of TYLCV have been found containing changes in the overlapping 
region of C1 and C4 (Ohnishi et al., 2016; Tomás et al., 2011). Recently, C1 was proven to be the Avr/effector of Ty-2 resistance (Shen et al., 2020). The ty-5 gene, encodes a tomato homolog of the protein Pelota (Lapidot et al., 2015). Pelota is a mRNA surveillance factor and involved in recycling of ribosomes during protein synthesis. It is postulated that ty-5, encoding a Pelota protein that is functionally compromised, hampers viral protein synthesis and as a consequence lowers viral replication. As an alternative, Pelota may interact with proteins involved in viral replication leading to resistance (Lapidot et al., 2015). It was first believed that Ty-1 and Ty-3, both derived from S. chilense, are two different genes (Ji et al., 2007; Zamir et al., 1994). Recent fine mapping and cloning of these genes have shown that the genes are allelic and that $T y-1 / T y-3$ codes for a RDR (Verlaan et al., 2013).

\section{Ty-1 and RNAi}

RDRs occur in plants, fungi, RNA viruses, protists, and some lower animals, but are not present in drosophila, mice and humans (Willmann et al., 2011). In Arabidopsis thaliana six different RDRs are identified and named RDR1 to -6 . They are divided in two classes, designated RDR $\alpha$ and RDR . RDR1, 2 and 6 belong to the RDR $\alpha$ class while RDR3, RDR4 and RDR5 belong to the RDRy class. Both classes can be

Table 1.3: Mapped Tomato genes for TYLCV resistance.

\begin{tabular}{|c|c|c|c|c|c|}
\hline Gene & Genetic source & Cloned & $\begin{array}{l}\text { Chromo- } \\
\text { some } \\
\text { location }\end{array}$ & Protein & Ref \\
\hline Тy-1/Тy-3 & S. chilense & Cloned & 6 & RDR & $\begin{array}{l}\text { (Verlaan et } \\
\text { al., 2013) }\end{array}$ \\
\hline Ty-2 & S. habrochaites & Cloned & 11 & NB-LRR & $\begin{array}{l}\text { (Yamaguchi } \\
\text { et al., 2018) }\end{array}$ \\
\hline Ty-4 & S. chilense & Not cloned & 3 & & $\begin{array}{l}\text { (Ji et al., } \\
2009)\end{array}$ \\
\hline ty-5 & $\begin{array}{l}\text { tomato breeding line TY172, } \\
\text { derived from four different } \\
\text { S.peruvianum accessions }\end{array}$ & Cloned & 4 & Pelota & $\begin{array}{l}\text { (Lapidot et } \\
\text { al., 2015) }\end{array}$ \\
\hline Ty-6 & S. chilense & Not cloned & 10 & & $\begin{array}{l}\text { (Hutton and } \\
\text { Scott, 2014) }\end{array}$ \\
\hline
\end{tabular}


distinguished based on the presence of a (distinct) catalytic domain. A phylogenetic analysis shows that $T y-1$ clusters with RDR3,4,5 and analysis of the amino acid sequence shows that $T y-1$ contains the atypical DFDGD motif in the catalytic domain characteristic for the RDRy class (Verlaan et al., 2013). RDR1, RDR2 and RDR6 have been well studied and assigned a role in the amplification of the RNAi pathway. Until 2013, when Ty-1 was cloned and demonstrated to code for an RDR of the gamma class, nothing was known about and no function assigned to any of the members of the RDR $y$ class. In light of the role of RDR $\alpha$ members in the amplification of RNAi, Butterbach et al. (Butterbach et al., 2014) postulated a similar role for Ty-1 in the amplification of RNAi, in specific related to a TGS response against geminiviruses and leading to reduced levels of TYLCV genome transcription and replication (Verlaan et al., 2013; Willmann et al., 2011). Their study revealed that Ty-1 supports the production of higher levels of TYLCV-specific siRNAs compared to those in the susceptible tomato cultivar Moneymaker (MM) and relative to virus titers. In addition, changes were observed in the genome distribution of the viral siRNAs i.e., in Ty-1 bearing plants the ratio of V1-derived siRNAs relative to C3-derived siRNAs increased. Furthermore, viral DNA collected from $T y-1$ bearing plants revealed a higher level of methylation of cytosine-residues at the start of the coding region of V1/CP compared to viral DNA collected from MM. These data altogether supported the idea that $T y-1$ confers resistance by enhancing TGS (Butterbach et al., 2014). With knowledge on the antiviral RNAi pathway and viral counter defence strategies, the authors also demonstrated that a mixed infection of CMV and TYLCV in Ty-1 bearing tomato plants compromised the resistance by $T y-1$ (Butterbach et al., 2014). This was likely due to the CMV $2 \mathrm{~b}$ RSS protein, earlier shown to be able to abrogate AGO4 during TGS (Duan et al., 2015; González et al., 2010; Hamera et al., 2012).

\section{Scope of the thesis}

At the onset of this research, the $T y-1$ gene was cloned and shown to encode a RDRY. Although initial studies showed that $T y-1$ confers resistance against TYLCV by enhancing the antiviral RNAi response, many details of its mode and site of action still remained unknown. The aim of this thesis was to investigate the mode of action of $T y-1$ in more detail. 
Initial studies already showed that besides TYLCV, Ty-1 also confers resistance against Tomato severe rugose virus (ToSRV), a bipartite New World begomovirus (Butterbach et al., 2014). Considering that RNAi provides a generic antiviral defence, it was anticipated that the resistance spectrum of $T y-1$ would extend to members from the other genera within the Geminiviridae family as well. To test this hypothesis, tomato lines bearing Ty-1 were challenged with TYLCV and BCTV, the type species of the curtovirus genus and transmitted by leafhoppers. In addition, the same viruses were tested on transgenic Nicotiana benthamiana ( $N$. benthamiana) and S. Lycopersicum plants expressing $T y-1$ to rule out the involvement of other introgressions (Chapter 2).

Previous studies also had shown that a mixed infection of TYLCV and CMV, a cytoplasmic replicating RNA virus, in Ty-1 bearing tomato plants compromised the resistance (Butterbach et al., 2014), likely due to the CMV 2b RSS protein (Duan et al., 2015; González et al., 2010; Hamera et al., 2012). Considering that monopartite Old World begomoviruses are often associated with betasatellites (Fig. 1.2), encoding a $\beta C 1$ TGS suppressor, it was anticipated that the presence of these satellites during a TYLCV infection would likely compromise $T y-1$ as well. To analyse this, $T y-1$ bearing plants were challenged with TYLCV in the absence and presence of a co-replicating betasatellite or trans complemented with $\beta C 1$ expressed from a Potato virus $X$ (PVX) replicon, and viral titers were determined. In addition, attempts were made to investigate whether the effectiveness of $T y-1$ was inversely proportional to the strength of the (viral-encoded) RNAi suppressor(s) encountered (Chapter 2).

Although Ty-1 was shown to enhance the antiviral RNAi response, nothing was known yet on the exact profile and genomic distribution of viral siRNAs produced and amplified. To provide a more detailed view on this, a library of small RNAs was made and sequence analysed from TYLCV challenged susceptible tomato MM and Ty-1 bearing tomato (Chapter $\mathbf{3}$ ).

Processing (P)-bodies are responsible for RNA decay and compete for aberrant RNA molecules (resulting from RISC activity) with the neighbouring siRNA bodies, where the amplification of the RNAi response takes place by RDR6. Impairing P-bodies has earlier been shown to induce a stronger antiviral PTGS response due to an increased 
availability of aberrant RNA substrate for RDR6 (De Alba et al., 2015; Gazzani et al., 2004; Gy et al., 2007; Hayashi et al., 2012; Thran et al., 2012; Vogel et al., 2011; Yu et al., 2015; Zhang et al., 2015). Whether impairing P-bodies would also contribute to a stronger antiviral RNAi response towards geminiviruses, which would indicate the potential presence of RDR in close proximity to P-bodies, was not known. To test this hypothesis $N$. benthamiana, in which P-bodies were silenced, were challenged with TYLCV and virus titers determined (Chapter 4).

To further extend on the experiments described in Chapter 4, Ty-1 was in situ localized in plant cells. To this end, Ty-1 was fused to GFP and using agroinfiltration transiently expressed in $N$. benthamiana. The spatial distribution of Ty-1 in the nucleus was further analysed relative to various nuclear body elements to indicate their relevance towards the antiviral defence response mediated by Ty-1. Using bimolecular fluorescence complementation, the interaction between Ty-1 and co-localizing host proteins was analysed (Chapter 5).

Finally, in Chapter 6 all the obtained results are summarised and discussed in light of current literature, and in respect to the relevance towards breeders and growers. Based on all results obtained from this study, a model is presented to summarize and explain the antiviral mode of action of Ty-1. 


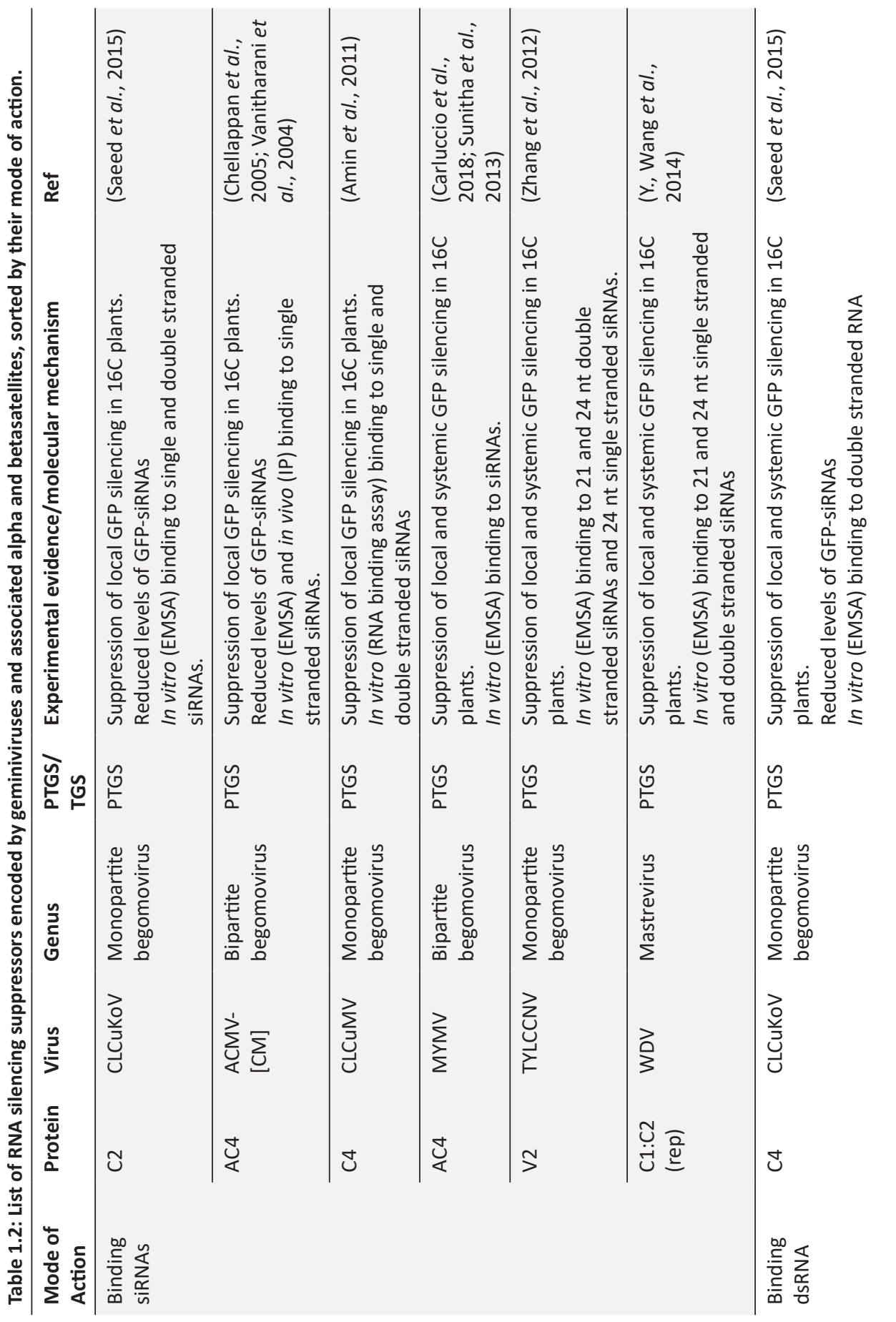




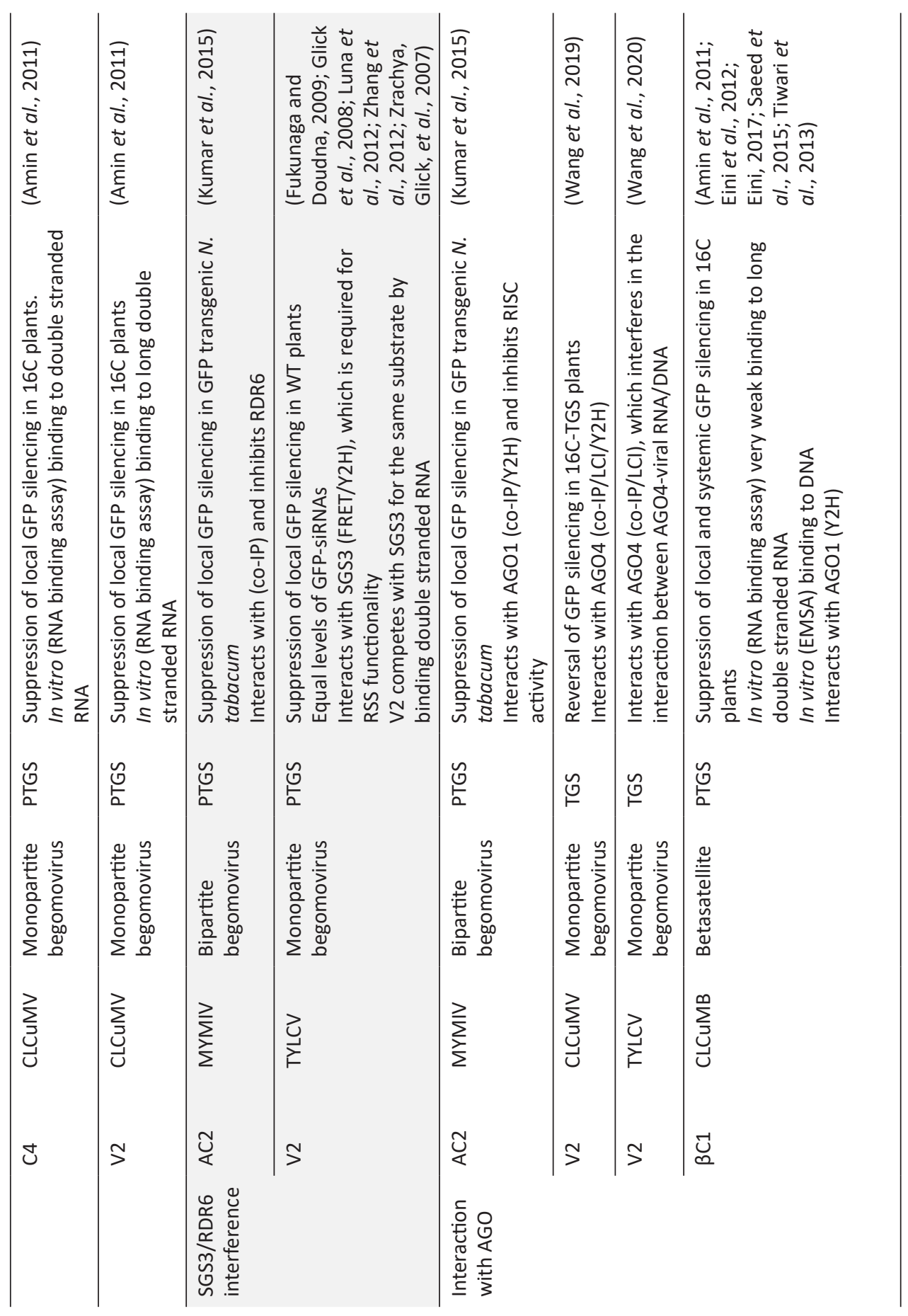




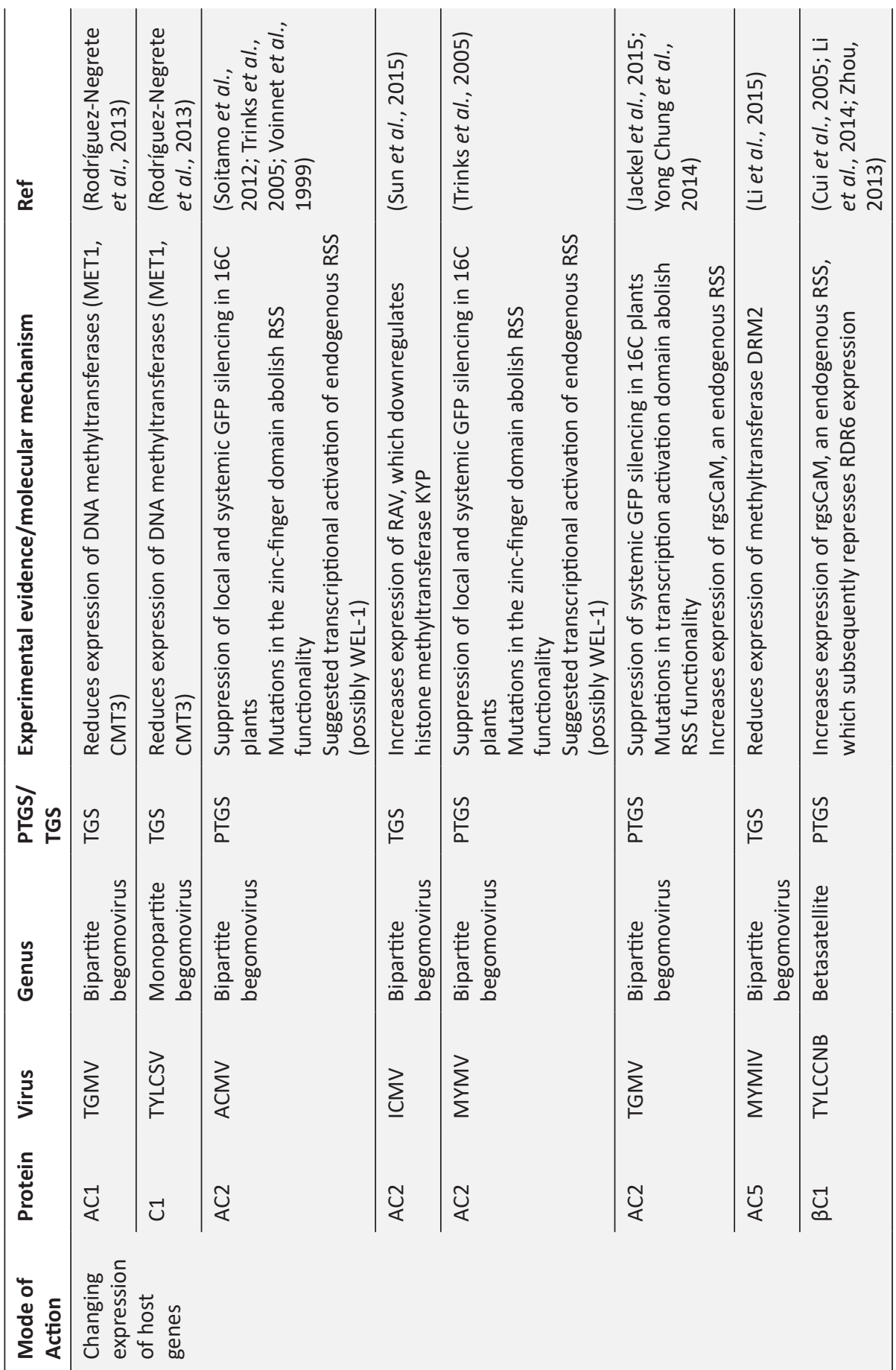




\begin{tabular}{|c|c|c|c|c|c|c|c|c|}
\hline 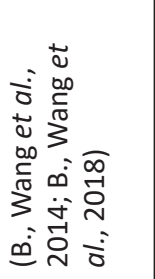 & 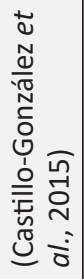 & 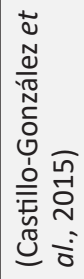 & 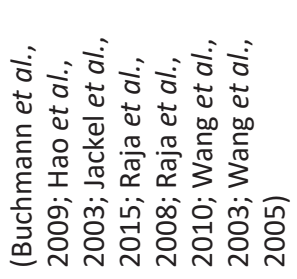 & 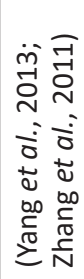 & 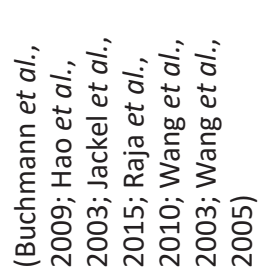 & 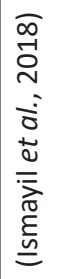 & 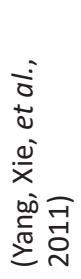 & 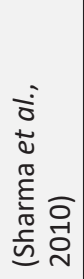 \\
\hline 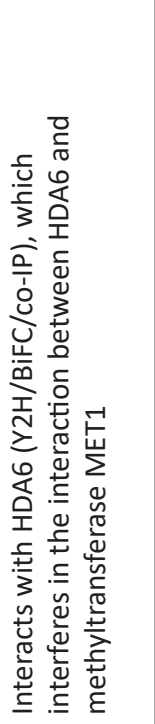 & 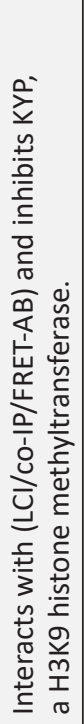 & 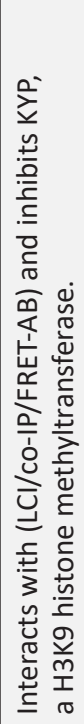 & 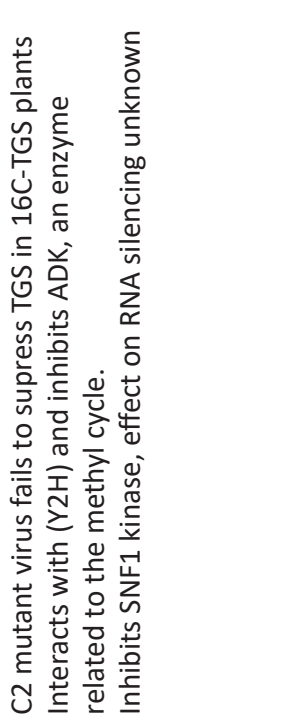 & 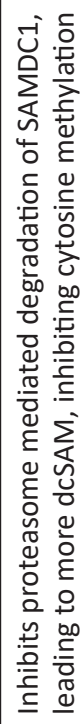 & 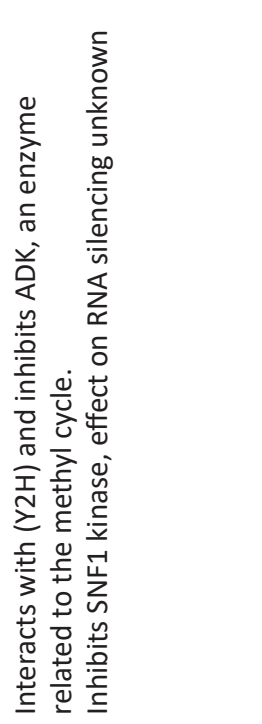 & 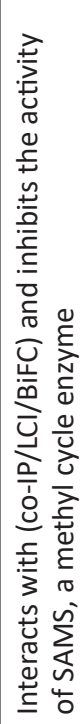 & 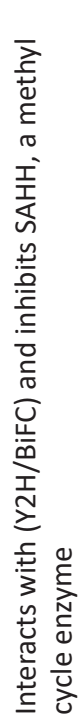 & 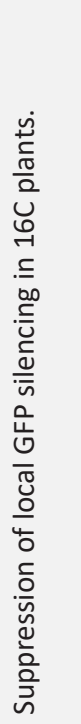 \\
\hline ળ్ & & ভ઼ & 心 & $\underset{ }{\mathscr{S}}$ & 心్ & U్ & 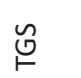 & 忍 \\
\hline 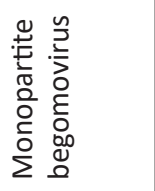 & 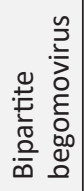 & 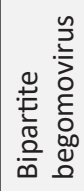 & 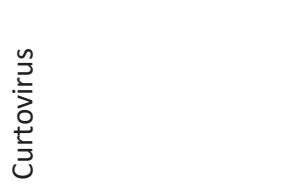 & $\begin{array}{l}\frac{n}{2} \\
\frac{2}{3} \\
\frac{5}{3} \\
3\end{array}$ & 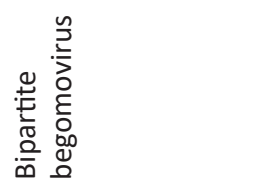 & 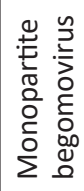 & 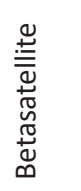 & 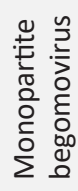 \\
\hline$\underset{己}{己}$ & 売 & 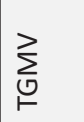 & 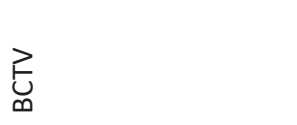 & 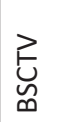 & 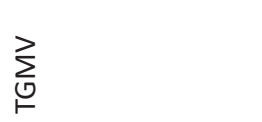 & 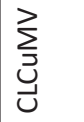 & 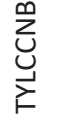 & $\sum_{\&}$ \\
\hline$\stackrel{\Upsilon}{>}$ & Uัષ & రัષ & $\mathcal{U}$ & U & ঠัष & J & 亡્ન & U \\
\hline 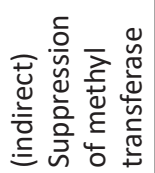 & 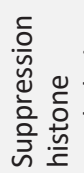 & & 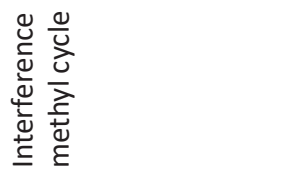 & & & & & $\begin{array}{l}\frac{5}{3} \\
0 \\
\frac{5}{5} \\
\frac{5}{5}\end{array}$ \\
\hline
\end{tabular}




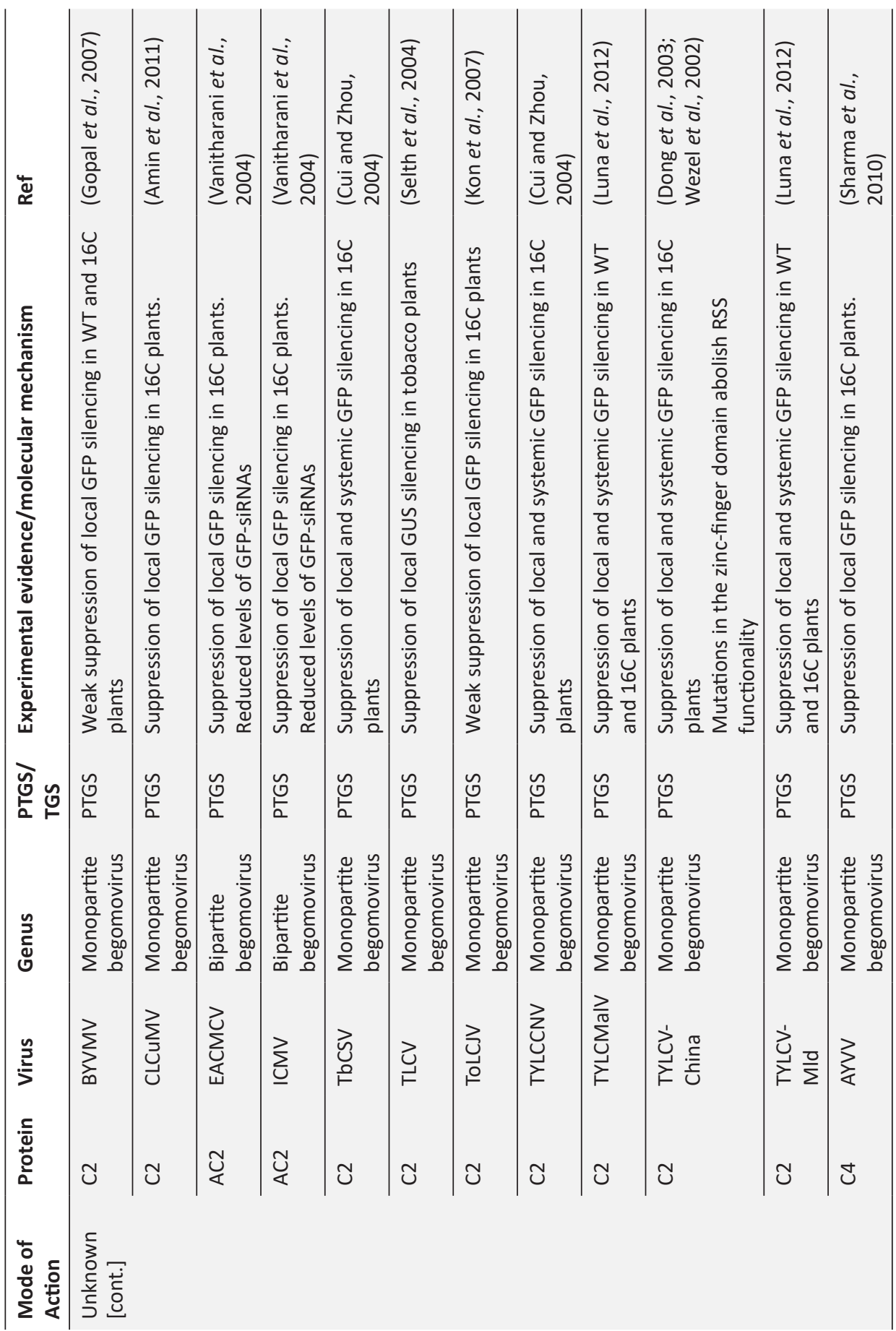




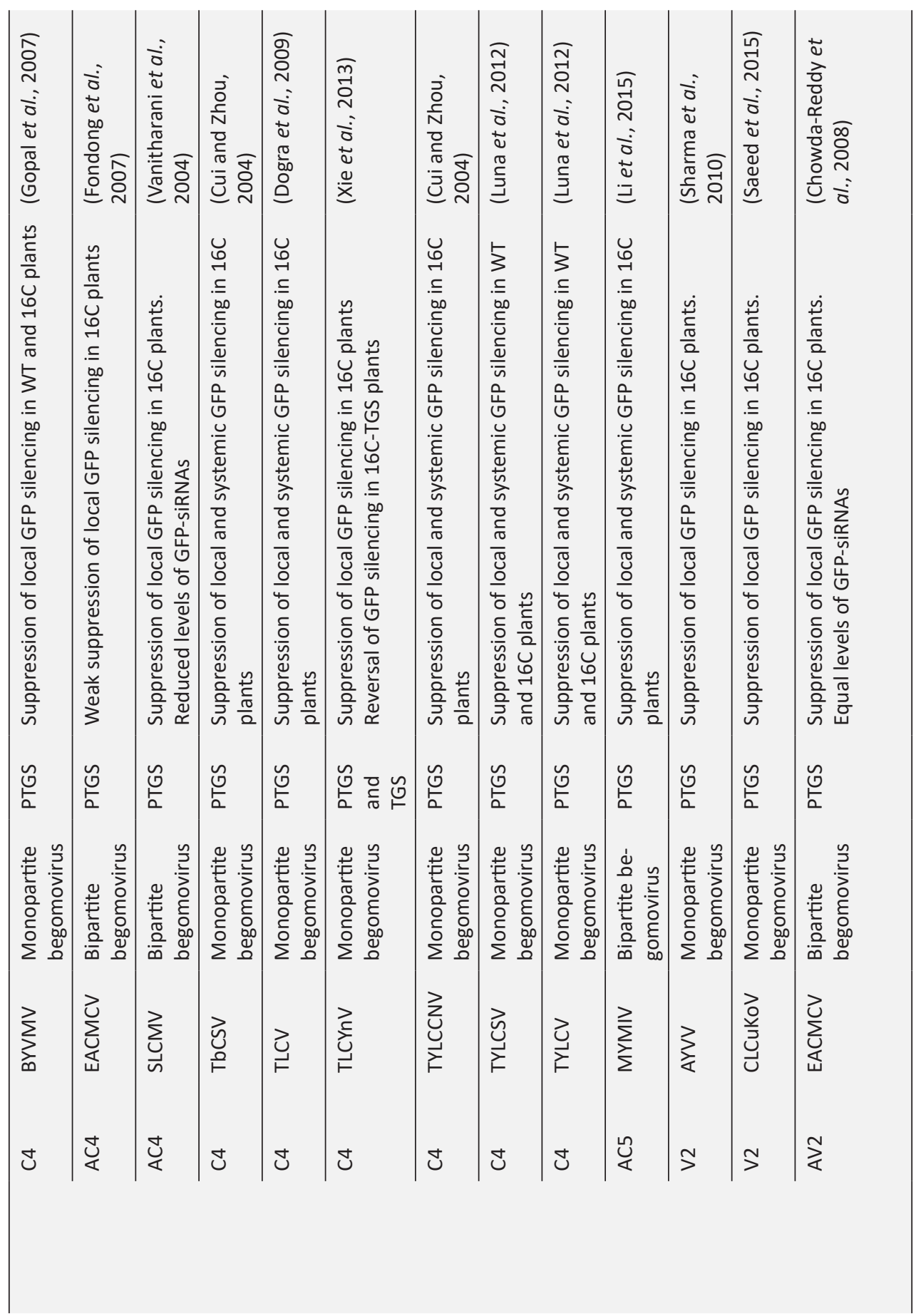




\begin{tabular}{|c|c|c|c|c|c|c|c|c|c|c|}
\hline$\overleftarrow{\mathscr{J}}$ & 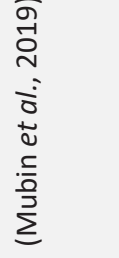 & 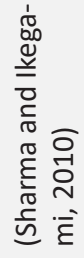 & 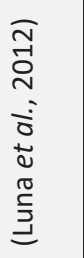 & 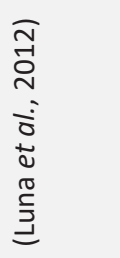 & 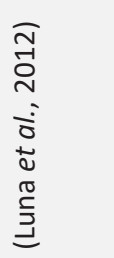 & 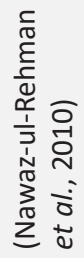 & 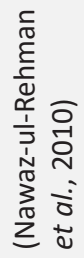 & 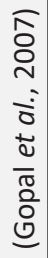 & 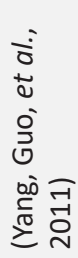 & 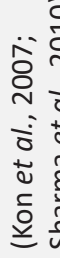 \\
\hline 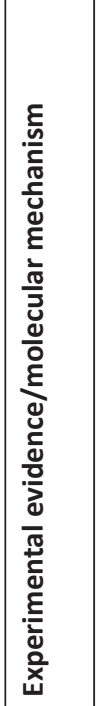 & 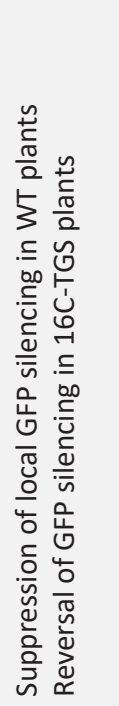 & 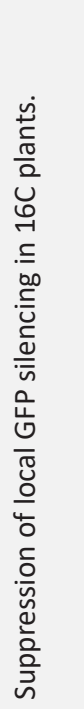 & 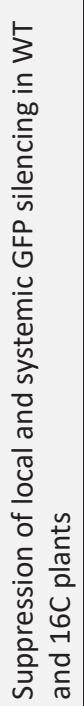 & 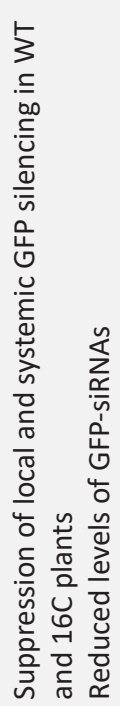 & 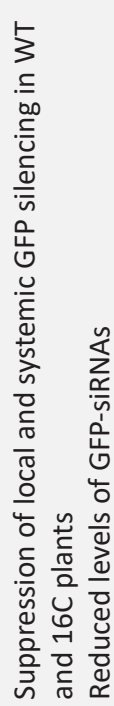 & 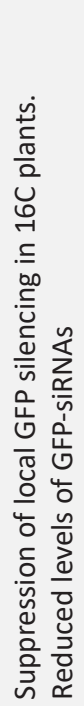 & 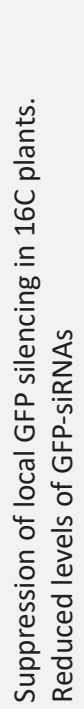 & 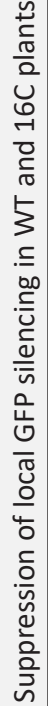 & 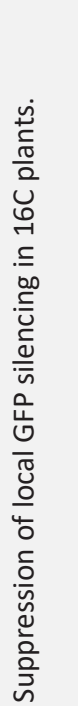 & 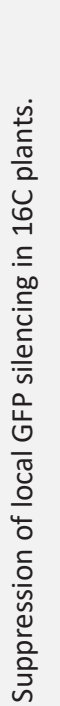 \\
\hline 忧 & 令 & 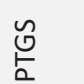 & 昏 & U్ & 代 & ़ֻ & ণิ & ల్ & ֻొ & 心్ \\
\hline S & 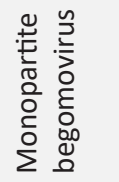 & 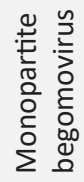 & 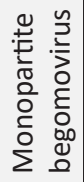 & 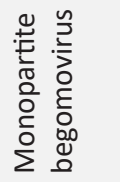 & 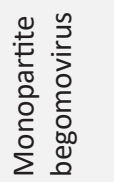 & 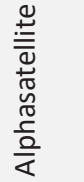 & 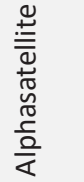 & 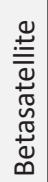 & 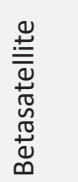 & 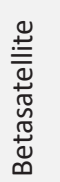 \\
\hline$\stackrel{n}{\underline{n}}$ & $\frac{3}{\widetilde{J}}$ & 궁 & 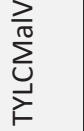 & 골 & $\stackrel{\sum_{i}}{\sum_{j}^{D}}$ & 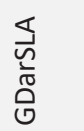 & 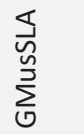 & $\sum_{i \infty}^{\infty}$ & $\begin{array}{l}\sum_{\mathcal{U}} \\
\text { 음 }\end{array}$ & $\begin{array}{l}\stackrel{9}{3} \\
\stackrel{0}{\circ}\end{array}$ \\
\hline के & $\stackrel{\sim}{>}$ & $\stackrel{\sim}{>}$ & 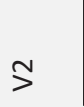 & $\stackrel{N}{>}$ & S & $\begin{array}{l}\frac{0}{0} \\
\frac{0}{\gamma}\end{array}$ & $\begin{array}{l}\frac{0}{0} \\
\frac{x}{\gamma}\end{array}$ & స్ & స్ & స్ \\
\hline 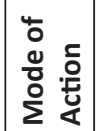 & 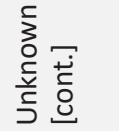 & & & & & & & & & \\
\hline
\end{tabular}




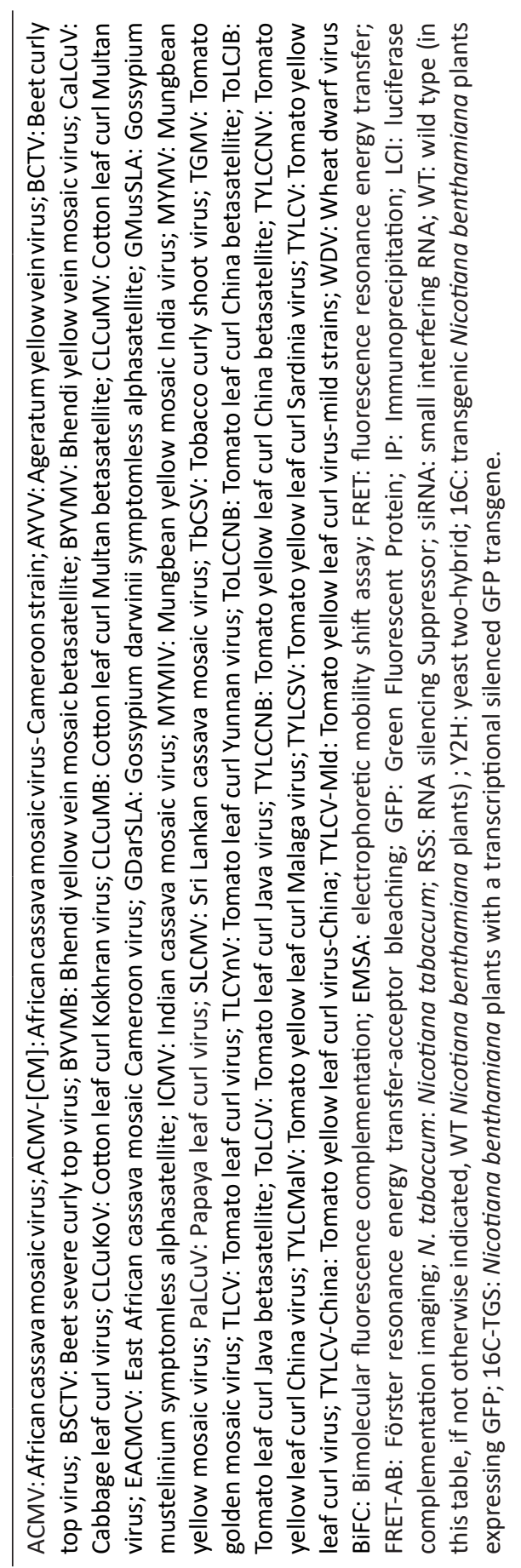





\section{Chapter 2}

\section{Ty-1, a universal resistance gene against geminiviruses that is compromised by co-replication of a betasatellite}

C.M. Voorburg*, Z. Yan*, M. Bergua-Vidal, A.M.A. Wolters, Y. Bai, R. Kormelink

* These authors contributed equally to this work

This chapter has been published in a slightly modified version as:

Voorburg, C.M., Yan, Z., Bergua-Vidal, M., Wolters, A.A., Bai, Y. and Kormelink, R. (2020) Ty-1, a universal resistance gene against geminiviruses that is compromised by co-replication of a betasatellite. Mol. Plant Pathol. 21, 160-172 


\section{Summary}

Tomato yellow leaf curl virus (TYLCV), a begomovirus, causes large yield losses and breeding for resistance is an effective way to combat this viral disease. The resistance gene Ty-1 codes for an RNA-dependent RNA polymerase (RDR) and is recently shown to enhance transcriptional gene silencing (TGS) of TYLCV. Whereas Ty-1 was earlier shown to also confer resistance to a bipartite begomovirus, here it is shown that $T y-1$ is likely generic to all geminiviruses. A tomato $T y-1$ introgression line, but also stable transformants of susceptible tomato cv Moneymaker (MM) and Nicotiana benthamiana ( $N$. benthamiana) expressing the $T y$-1 gene, exhibited resistance to begomoviruses as well as to the distinct, leafhopper transmitted beet curly top virus (BCTV), a curtovirus. Stable Ty-1 transformants of $N$. benthamiana and tomato showed less symptoms and reduced viral titers upon infection compared to wildtype plants. TYLCV infections in wildtype $N$. benthamiana plants in the additional presence of a betasatellite led to increased symptom severity and a consistent, slightly lowered virus titer relative to the high averaged levels seen in the absence of the betasatellite. On the other hand, in Ty-1 transformed $N$. benthamiana viral titers increased in the presence of the betasatellite. The same was observed when these Ty-1 encoding plants were challenged with TYLCV and a potato virus X (PVX) construct expressing the RNA interference suppressor protein $\beta C 1$ encoded by the betasatellite. The resistance spectrum of $T y-1$ and the durability of the resistance will be discussed in light of antiviral RNA interference and viral counter defence strategies. 


\section{Introduction}

Tomato yellow leaf curl virus (TYLCV) is the representative of the Old World monopartite begomoviruses within the family Geminiviridae. The virus belongs to the most devastating plant viruses worldwide and causes major losses in economically important crops like tomato. The virus mainly occurs in the (sub) tropical and Mediterranean regions worldwide due to the distribution of its vector the whitefly Bemisia tabaci (B. tabaci) (Moriones and Navas-Castillo, 2000). TYLCV, and all monopartite begomoviruses, have a single, circular single stranded (ss) DNA genome of $\sim 2.7 \mathrm{~kb}$ in size. In the field, monopartite begomoviruses are frequently observed with co-replicating alpha- or betasatellites (Nawaz-ul-Rehman and Fauquet, 2009). Both satellites are approximately half the size of begomovirus genomes. While less is known on alphasatellites and their role in pathogenesis of begomoviruses, co-replication of betasatellites often leads to more severe disease symptoms (Zhou, 2013). Therefore, betasatellites are regarded as pathogenicity determinants. Betasatellites code for one single protein called $\beta C 1$ that is known to suppress the antiviral defence mechanism transcriptional gene silencing (TGS) (Yang, Xie, et al., 2011; Zhou, 2013).

Because the vector $B$. tabaci is difficult to control, the most effective way to combat TYLCV infections is to breed for resistance. So far six resistance genes are known namely $T y-1$ to $T y-6$ and some of these are widely used for introgression breeding. Five of these genes are derived from wild tomato species: $T y-1, T y-3, T y-4$ and $T y-6$ are from Solanum chilense (S. chilense), Ty-2 from $S$. habrochaites, while ty-5 was identified in an old commercial tomato cultivar Tyking (Hanson et al., 2006; Hutton et al., 2012; Hutton and Scott, 2014; Ji et al., 2007; Ji et al., 2009; Lapidot et al., 2015; Zamir et al., 1994). Upon viral challenge plants containing any of those Ty genes do not seem to completely abolish infection as reduced virus titers are still observed in comparison to susceptible cultivars, nor is a hypersensitive response seen (Castro et al., 2005; Maruthi et al., 2003; Picó et al., 1999; Picó et al., 2000). In the past years, several of those genes have been cloned and characterized, only $T y-4$ and $T y-6$ are not. $T y-2$ encodes a NB-LRR protein, while the $t y-5$ gene is encoding a mRNA surveillance factor and contains a mutation that hampers viral protein translation (Lapidot et al., 2015; Yamaguchi et al., 2018). Ty-1 and Ty-3 are 
allelic, and code for a RNA-dependent RNA polymerase (RDR) from the $\boldsymbol{\gamma}$-class and distinct from the well-established RDRs from the $\alpha$-class with recognized functions in the amplification of the antiviral RNA interference (RNAi) response (Verlaan et al., 2013). Until recently, no function was assigned to any of the RDRs from the $y$-class, but with the unravelling of $T y-1$ a new class of resistance genes was unveiled.

Besides a major role in gene regulation and chromosome dynamics, RNAi (also named RNA silencing) presents a major antiviral defence mechanism in plants (Ding and Voinnet, 2007). The mechanism is induced by double stranded (ds) RNA molecules, which arise from viral replicative intermediates or secondary RNA folding structures in genomic or messenger RNA molecules. After their processing by Dicerlike proteins (DCL) into small interfering (si) RNA molecules of 21-24 nucleotide (nt), one strand of the siRNA is uploaded into a RNA-Induced Silencing Complex (RISC), leading to its activation (Hammond, 2005). When this complex is loaded with a 21 nt siRNA strand and contains an Argonaute 1 (AGO1) core component, it is able to target RNA molecules with sequence complementarity to the siRNA strand, leading to their degradation or translational arrest (Mallory and Vaucheret, 2010). This process is generally referred to as post-transcriptional gene silencing (PTGS). On the other hand, AGO4 containing RISC is loaded with a 24 nt siRNA strand. This complex targets cytosine methyltransferases to complementary DNA molecules, causing cytosine methylation within the target sequence. This methylation leads to TGS of the DNA sequence (Mallory and Vaucheret, 2010).

The RNAi response in plants, in contrast to insects and animals, is being amplified by RDRs (Donaire et al., 2008; Wang et al., 2010). During this process aberrant RNA molecules, resulting from the first cleavage by the slicer activity of AGO1, are recognized by RDRs and converted into more dsRNA molecules of the target sequence. Their processing by DCLs produces a second generation of siRNAs. Due to this amplification a strong (antiviral) RNAi response is mounted. In the Arabidopsis thaliana genome, six RDR genes have been annotated, encoding RDR 1 to -6 . From those, RDR1, -2 and -6 represent the $\alpha$-class, whereas RDR3, -4 and -5 fall in the $\gamma$-class (Willmann et al., 2011). Silencing of RDR1 and RDR6 from the $\alpha$-class reduces the antiviral PTGS response and makes plants highly susceptible to RNA viruses, but the RNAi response against geminiviruses does not seem to be affected much by 
silencing of these RDRs (Aregger et al., 2012; Raja et al., 2008; Yu et al., 2003). This finding not only pointed to the involvement of other RDRs in the RNAi response against geminiviruses, but also supported the idea that the TGS pathway is the most important RNAi pathway against geminiviruses. In agreement with this hypothesis are findings showing that plants knocked out for proteins from the TGS pathway are hypersusceptible to geminivirus infection (Jackel et al., 2016; Raja et al., 2008). After the identification of $T y-1$ as an RDR $y$-class member, its involvement in the RNAi amplification was demonstrated by an increase of siRNA production and an elevated rate of cytosine methylation in viral DNA collected from TYLCV-challenged Ty-1 tomato plants (Butterbach et al., 2014).

While the Ty-1 resistance gene is generally deployed to combat TYLCV, it has been shown to confer resistance to begomoviruses other than TYLCV (Barbieri et al., 2010; Butterbach et al., 2014; Pietersen and Smith, 2002; Prasanna et al., 2015; Shahid et al., 2013). However, whether the resistance is also effective against members of other genera, e.g. the Curtovirus genus, is still unknown. Furthermore, all previous studies on $T y$-1 so far have been conducted using introgression lines and therefore it still remained to be questioned whether the resistance is solely due to the expression of $T y-1$ or could involve other chromosomal introgression(s). Lastly, the earlier study by Butterbach et al. (2014) pointed towards an "Achilles' heel" of Ty-1 resistance, namely suppression of TGS by viral RNAi suppressor proteins. In that study the Ty-1 resistance against TYLCV was compromised by a co-infection with cucumber mosaic virus (CMV) (Butterbach et al., 2014). This effect was attributed to the CMV encoded RNAi suppressor $2 b$ that was shown to inhibit AGO4 activity during TGS (González et al., 2010; Hamera et al., 2012). Since betasatellites encode suppressors of TGS, the compromising nature of betasatellites, by their encoded $\beta C 1$ protein, on $T y-1$ resistance could be anticipated.

Here it is shown that $T y-1$ from a tomato introgression line, but also after stable transformation into susceptible tomato Moneymaker (MM) and Nicotiana benthamiana ( $N$. benthamiana) confers resistance to a completely distinct leafhopper transmitted curtovirus, in casu beet curly top virus (BCTV). Furthermore, TYLCV resistance in transgenic Ty-1 plants is compromised not only by a co-replicating 
betasatellite, but also by transient co-expression of its encoded TGS suppressor protein only.

\section{Results}

\section{Broad spectrum resistance against geminiviruses in $T y-1$ introgression line}

Earlier, $T y$-1 has been shown to confer resistance not only to the monopartite TYLCV, but also to the bipartite begomovirus tomato severe rugose virus (ToSRV) (Butterbach et al., 2014). In light of its role in the amplification of RNAi, a mechanism being antiviral and generic to all viruses, it was anticipated that $T y-1$ resistance would not be restricted to species from the TYLCV cluster. To test this hypothesis and determine the resistance spectrum of $T y-1$, a $T y-1$ breeding line was challenged with various TYLCV-like viral species, i.e. a TYLCV isolate from Spain and China (both belonging to the TYLCV Israel species), tomato yellow leaf curl Sardinia virus (TYLCSV), as well as a completely distinct geminivirus, namely the leafhopper transmitted BCTV, which is a representative of the Curtovirus genus. Plants were monitored for seven weeks by scoring disease symptoms and viral titers were determined. Whereas the susceptible control S. lycopersicum cv. MM showed severe disease symptoms after a challenge with all (curto- and begomo-) viruses, the Ty-1 breeding line remained (almost completely) symptomless upon a challenge with the TYLCV Almeria isolate, TYLCV-[CN:SH2], TYLCSV and BCTV (Fig. 2.1A). Subsequently, the virus titers were determined via qPCR and the fold difference in titer between the susceptible MM plants and the $T y-1$ introgression line calculated using the DeltaDeltaCt method (Livak and Schmittgen, 2001). While in MM plants titers were high for all four viruses (Ct values between 14-16), their amounts were significantly reduced in Ty-1 introgression lines (Fig. 2.1B). Ty-1 resistance seemed most effective to TYLCSV and TYLCV-[CN:SH2], for which (hardly) no virus was detected in Ty-1 plants (Ct values between 32-34). TYLCV and BCTV were still clearly detected in Ty-1 plants (Ct values between 18-20), but TYLCV titers dropped $\sim 18$ fold and BCTV titers $\sim 3$ fold in Ty-1 plants compared to MM plants (Fig. 2.1B). 
A.
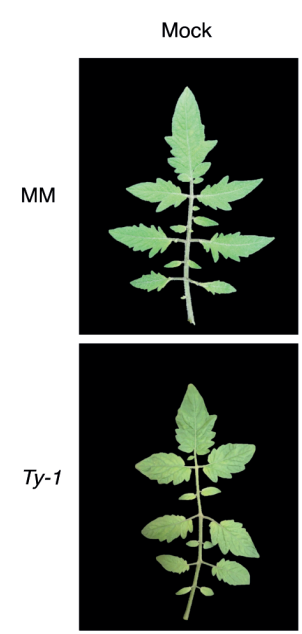

B.

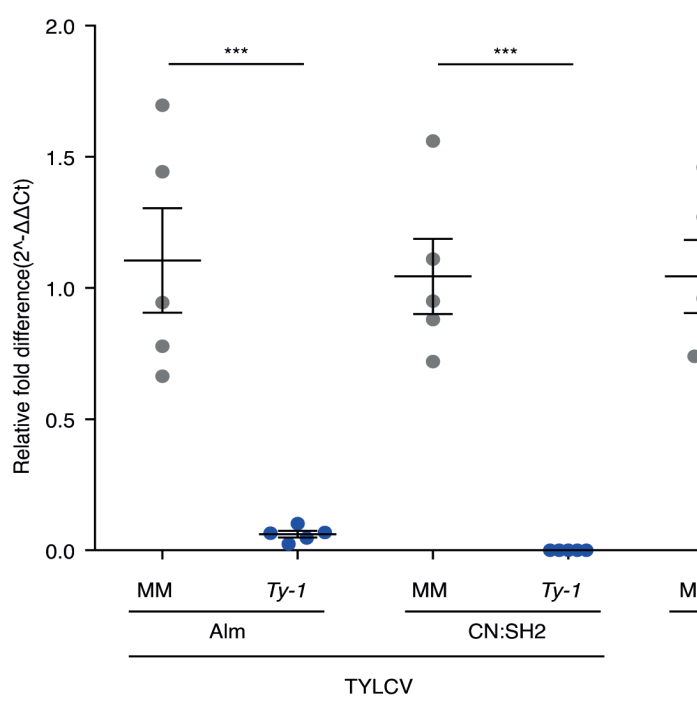

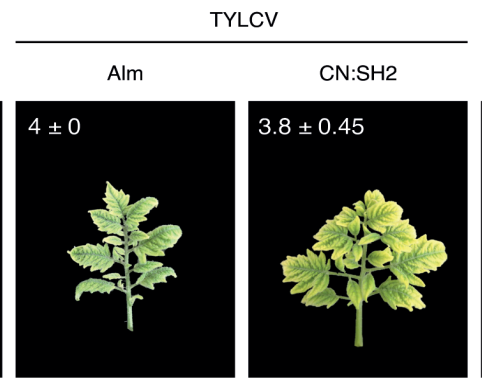
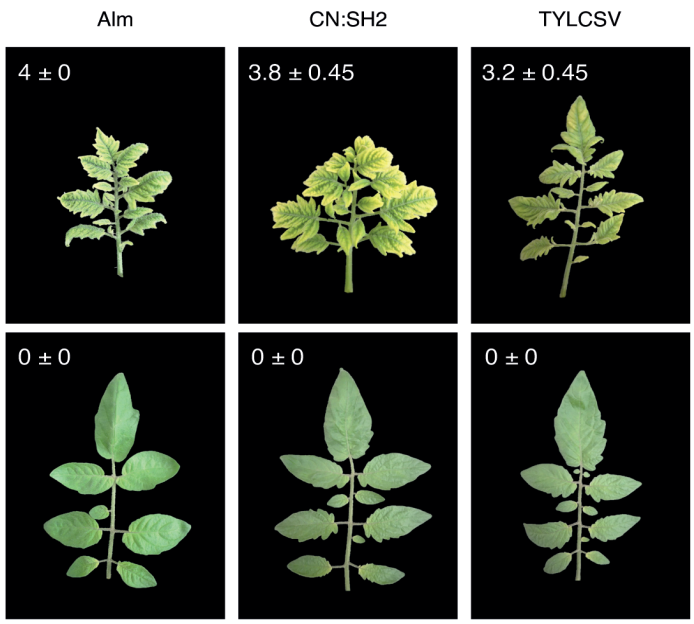

Viral titer
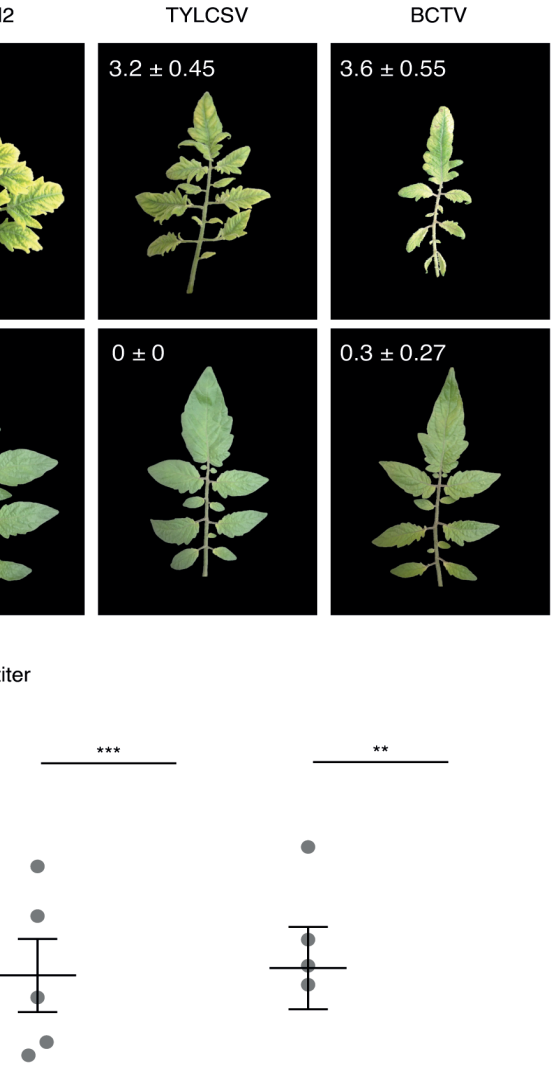

-

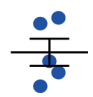

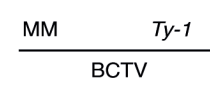

Figure 2.1: The Ty-1 introgression line is resistant against a broad range of geminiviruses.

A. Symptoms upon infection with either mock, TYLCV Almeria isolate (AIm), TYLCV-[CN:SH2], TYLCSV, and BCTV in susceptible control (S. lycopersicum cv. MM) and Ty-1 introgression plants at $50 \mathrm{dpi}$. In the left upper corners, the symptom scores are depicted as mean of five biological replicates \pm the standard deviation. B. Virus titer quantification of TYLCV-AIm, TYLCV-[CN:SH2], TYLCSV, and BCTV in MM and the Ty- 1 introgression line. Values were normalized relative to tomato EF1 $\alpha$ and calibrated to the levels in MM plants (set to 1 ). Dots represent the relative virus titer of individual plants. Lines represent means and standard error of the mean of biological replicates. Asterisks indicate significant differences between $T y-1$ introgression line and $\mathrm{MM}$ according to one-way analysis of variance $\left({ }^{*}=p<0.05,{ }^{* *}=p<0.01, * * *=p<0.001\right)$. 


\section{Transgenic expression of $\mathrm{Ty}-1$ in tomato confers broad spectrum resistance to geminiviruses}

To rule out that any other chromosomal introgression(s) would be involved, and to ensure that the broad spectrum resistance was solely due to the expression of the Ty-1 gene, susceptible tomato MM was transformed with a copy of the Ty-1 gene for a functional complementation study. Agrobacterium-mediated transformation of MM plants with a 35S promoter driven $T y-1$ construct resulted in nine primary transformants $\left(T_{1}\right)$. After selfing of the $T_{1}$ plants a $T_{2}$ progeny was obtained that was challenged with TYLCV and monitored for phenotypic responses. In all the subsequent experiments, the Almeria isolate of TYLCV was used as representative of the Begomovirus genus, as was also done in previous $T y-1$ research (Butterbach et al., 2014; Verlaan et al., 2013). Several $T_{2}$ families showed a clear segregation for TYLCV resistance (mild symptoms) correlated to the presence of the Ty-1 transgene, as scored by a positive PCR amplification of the NPTII gene cassette and $T y-1$ insertion (data not shown). $T_{2}$ plants showing resistance to TYLCV were used for selfing to produce a $\mathrm{T}_{3}$ generation. After another round of TYLCV challenges two $T_{3}$ families were selected from which all seedlings exhibited TYLCV resistance. These two families had originated from two different primary transformants. A reverse transcription (RT)-qPCR analysis on samples collected from these two families revealed a significant increase in the expression level of $T y-1$, which may vary between lines depending on integration site or number (Fig. 2.2A). The relative fold difference was calculated via the $2^{\wedge}$-DeltaDeltaCt method, showing a 20 - to 30 fold increase in $T y-1$ expression levels in $T y-1$ transformed lines relative to the $t y-1$ allele (detected by the same primer set) from susceptible MM plants. Furthermore, a $\sim 10$ fold reduction was observed in TYLCV titers in the transgenic lines relative to the untransformed, susceptible MM plants (Fig. 2.2B). $\mathrm{T}_{3}$ individuals from these two lines that exhibited significantly higher $T y$-1 expression levels and reduced virus accumulation were selected and selfed to produce two homozygous $T_{4}$ lines for further analysis ( $T_{4}$ line 1 and 2 ).

To complement and support the findings on the resistance spectrum of $T y-1$, batches of 10 seedlings from the stable $T y$ - 1 transformants ( $T_{4}$ line 1 and 2 ) were challenged with TYLCV and BCTV and monitored for their phenotypic responses. 


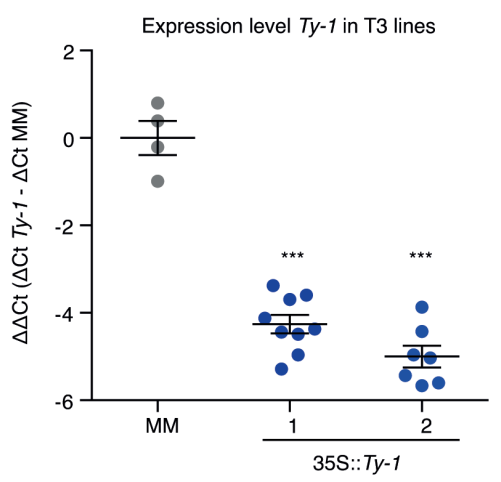

B.

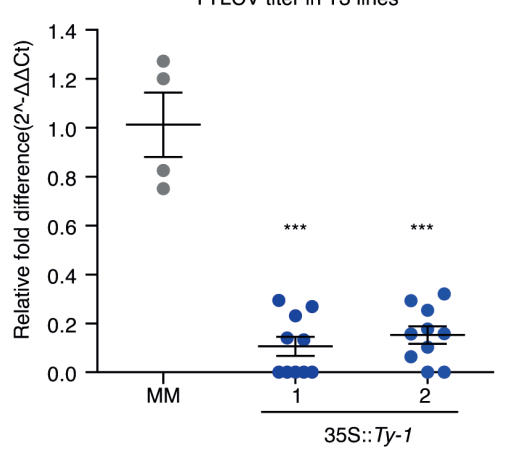

C.
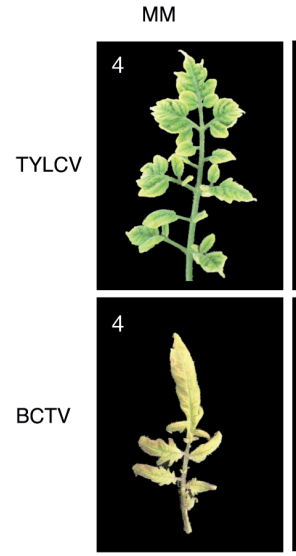

D.

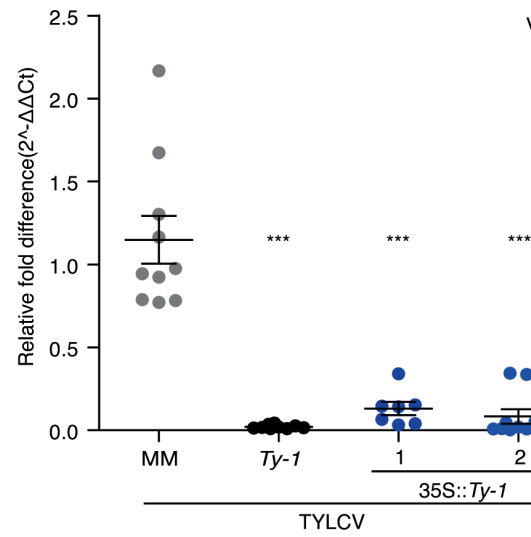

Ty-1 T4 line 1
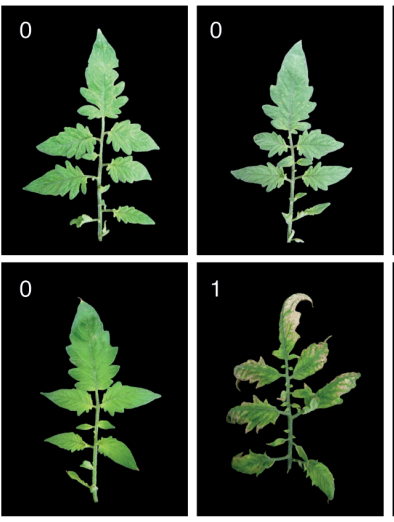

Viral titer in T4 lines
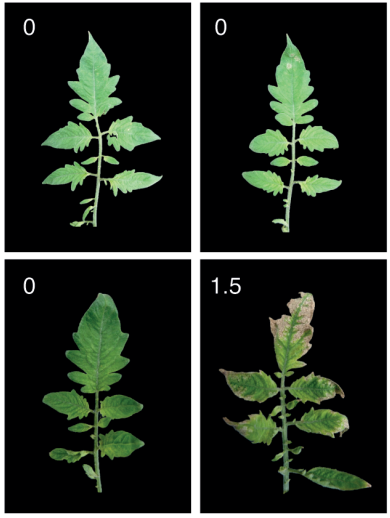

Figure 2.2: Transgenic tomato plants expressing Ty-1 are resistant against both BCTV and TYLCV.

For resistant control (Ty-1 introgression line) and susceptible control (S. lycopersicum cv. MM), five plants were included; ten plants of each transgenic line were tested. In all graphs, lines represent means and standard error of the mean of biological replicates, dots represent individual plants. A. Transcript levels of $T y-1$ in $T_{3}$ families. Values were normalized relative to EF1 $\alpha$. The DeltaDeltaCt values (DeltaCt $T y-1$ (Ct Ty-1 Ct EF1 $\alpha$ ) - DeltaCt MM (Ct Ty-1-Ct EF1 $\alpha)$ ) are depicted, which represent Ty-1 expression relative to the level 
of the susceptible ty-1 allele in untransformed MM plants. Asterisks indicate significant differences between $\mathrm{T}_{3}$ and untransformed MM plants according to one-way analysis of variance $(* * *=p<0.001)$. B. Virus titer quantification in $\mathrm{T}_{3}$ progeny of $T y-1$ transformants. Values were normalized relative to EF1 $\alpha$ and calibrated to the levels in untransformed MM plants, which were set to 1 . Asterisks indicate significant differences between $\mathrm{T}_{3}$ and untransformed MM plants according to one-way analysis of variance $\left({ }^{* * *}=\mathrm{p}<0.001\right)$. C. Symptoms upon infection with either mock, TYLCV and BCTV in MM and in different individuals of two $\mathrm{T}_{4}$ families of $T y-1$ transformed tomato plants at $50 \mathrm{dpi}$. In the upper left corners, the disease symptom scores are depicted. D. Virus titer quantification in plants from $2 \mathrm{~T}_{4}$ families (35S::Ty-1), a Ty-1 introgression line and untransformed MM. Values are calibrated to tomato EF1 $\alpha$ and the fold difference was calculated compared to (susceptible) MM plants inoculated with TYLCV or BCTV (set to 1). Asterisks represent significant differences to the TYLCV or BCTV challenged MM samples according to one-way analysis of variance $\left.{ }^{* * *}=p<0.001\right)$.

All seedlings from both lines showed resistance against TYLCV and displayed no disease symptoms (Fig. 2.2C). However, upon a challenge with BCTV, one out of ten plants from $\mathrm{T}_{4}$ line 1 , and four out of ten from $\mathrm{T}_{4}$ line 2 ended up showing mild BCTV symptoms (Fig. 2.2C). Systemic leaves were collected $50 \mathrm{dpi}$ and viral titers of both TYLCV and BCTV were determined by $\mathrm{PPCR}$ in both lines and compared to the titers in $\mathrm{MM}$ and the $T y-1$ tomato introgression line. The results showed comparable levels of viral DNA accumulation for TYLCV and BCTV in both the Ty-1 introgression line and $T y-1$ transgenic lines, and clearly reduced relative to the titers obtained from susceptible MM. However, the reduction in BCTV accumulation in both $\mathrm{T}_{4}$ lines was less profound than that of TYLCV (Fig. 2.2D). Plants from the two $\mathrm{T}_{4}$ lines that exhibited mild BCTV symptoms showed relatively higher BCTV titers compared to plants that remained symptomless.

\section{Transgenic expression of $T y-1$ in $\mathbf{N}$. benthamiana confers resistance to TYLCV and BCTV}

To further substantiate the findings on broad spectrum resistance conferred by $T y-1$, and to test whether the $T y-1$ resistance gene also functions in other Solanaceae species, stable transformants of $N$. benthamiana plants were generated expressing Ty-1 driven by a $35 \mathrm{~S}$ promoter. In analogy to the transformation of tomato MM (previous section), stable transformants were selected based on a TYLCV resistant phenotype and selfed until $\mathrm{T}_{4}$. Expression levels of $T y$-1 were determined by RT-qPCR in four transgenic lines selected from two independent transformations (Fig. 2.3A). The four transgenic lines showed a comparable high level of expression 
of $T y-1$. Whereas values for $\mathrm{Ct}$ were in the range of $\sim 25$ for all four transgenic lines, no signal was obtained from wildtype (WT) $N$. benthamiana samples, due to the absence of a ty-1 homolog in WT $N$. benthamiana that could be amplified by the primers used. The $\mathrm{Ct}$ values were normalised to the expression of the housekeeping gene Elongation Factor $1 \alpha(\mathrm{EF} 1 \alpha)$, and the DeltaDeltaCt values were calculated to compare Ty-1 transgenic plants with WT N. benthamiana, showing clear and high expression levels in Ty-1 plants. Upon infection with TYLCV no clear symptoms were observed in all four lines (Fig. 2.3B), and when virus titers were determined a 100 -fold reduction was observed in the transgenic lines compared to WT $N$. benthamiana plants (Fig. 2.3C).

When the stable $T y-1 \mathrm{~N}$. benthamiana transformants were challenged with BCTV only a slight curling and chlorosis (average symptom score 1.5) was observed at $18 \mathrm{dpi}$, whereas WT $N$. benthamiana revealed a strong curling and major chlorosis of systemic leaves (average symptom score 3.8) (Fig. 2.3B). BCTV titers showed a $\sim 15$-fold reduction relative to the titers obtained from susceptible $N$. benthamiana, which was smaller than the difference in TYLCV titers (between 70 and 180-fold) (Fig. 2.3C). This smaller effect on BCTV compared to TYLCV was consistently observed during repeated experiments and resembled the observations in the $T y-1$ tomato introgression line and transgenic tomato.

\section{Co-replication of a betasatellite compromises resistance by $T y-1$}

Earlier, a co-infection of CMV was shown to compromise Ty-1 resistance (Butterbach et al., 2014), likely due to the abrogation of AGO4 activity by the CMV $2 b$ RNAi suppressor protein. Under natural field conditions TYLCV is often observed with coreplicating betasatellites, elements that encode suppressors of TGS (Yang, Xie, et al., 2011). To test whether betasatellites also compromise $T y-1$ resistance, susceptible $\mathrm{MM}$ and $T y-1$ introgression tomato plants were co-infected with TYLCV and an ageratum yellow vein virus associated betasatellite (AYVB) via agroinfiltration. In parallel, WT $N$. benthamiana plants were infected with either TYLCV alone, or in combination with the betasatellite. At $21 \mathrm{dpi}$, no differences in viral symptoms were observed between TYLCV singly-infected and TYLCV plus betasatellite co-infected 
A.

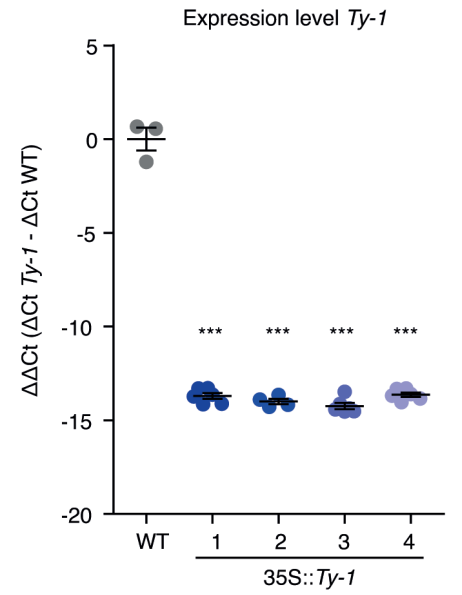

B.

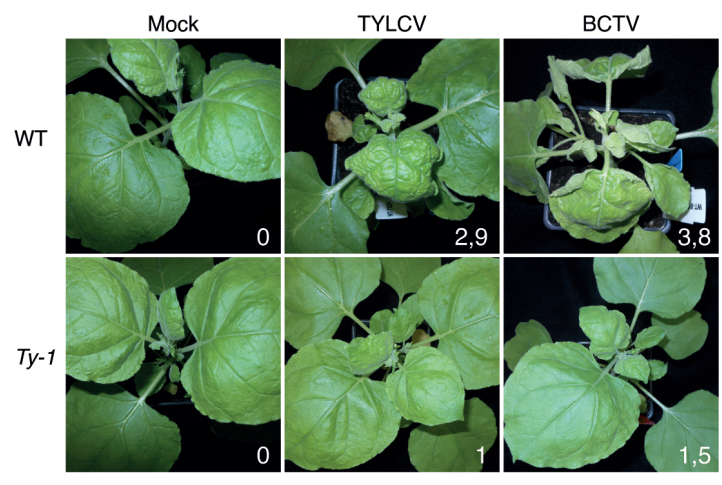

C.

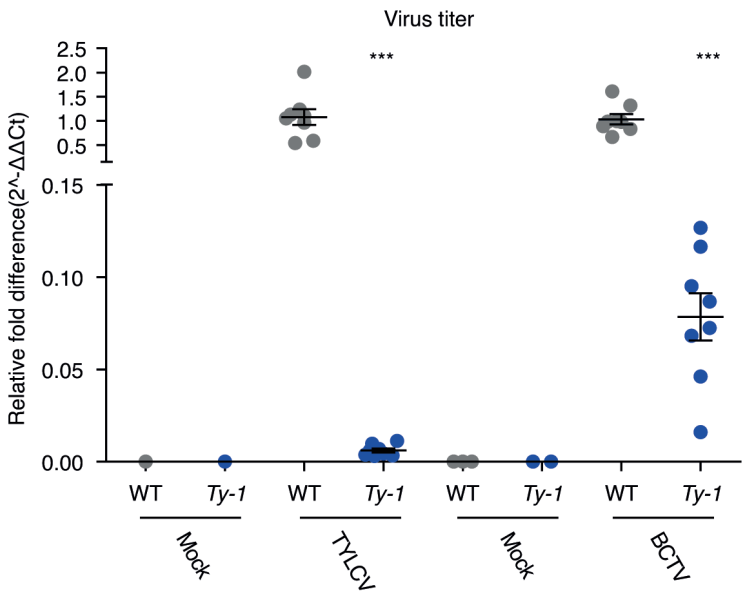

Figure 2.3: $\mathbf{T y}-1$ transgenic $\boldsymbol{N}$. benthamiana plants are resistant to TYLCV and BCTV.

In all graphs, every point represents one plant and lines represent means and standard error of the mean of biological replicates. A. Four independent transformant lines were generated and the expression level of Ty-1 was measured. Values were normalized relative to EF1 $\alpha$. The DeltaDeltaCt values (DeltaCt Ty-1 (Ct Ty-1 - Ct EF1 $\alpha$ ) - DeltaCt WT (Ct Ty-1 - Ct EF1 $\alpha)$ ) are depicted, which represent Ty-1 expression relative to WT plants. Asterisks indicate significant differences between WT plants and Ty-1 transformations according to one-way analysis of variance $(* * *=p<0.001)$. B. Symptoms upon infection with either mock, TYLCV or BCTV in WT and Ty-1 transgenic $N$. benthamiana plants. Pictures were taken at 18 dpi and the symptom scores are depicted in the lower right corners. C. Virus titer quantification in WT and Ty-1 transgenic $N$. benthamiana plants upon challenge with BCTV or TYLCV. 18 days post BCTV/TYLCV infection, systemic leaves were harvested. Total DNA was isolated and viral titers were measured compared to the presence of genomic DNA with primers amplifying the gene for 25S rRNA. The relative fold difference is depicted, which is calculated via the $2^{\wedge}$-DeltaDeltaCt method (WT infected plants set to 1 ). Please note the $y$-axis is split to show the low titers. Asterisks indicate significant differences between WT plants and Ty-1 transformants according to one-way analysis of variance $(* * *=p<0.001)$. 
tomato plants (results not shown). However, PCR analysis of systemically infected tomato leaves for the presence of the betasatellite turned out negative (data not shown), indicating that the betasatellite did not co-replicate with TYLCV in tomato. In contrast to tomato, at 19 dpi curling and yellowing of systemic leaves was more severe in $N$. benthamiana plants co-infected with TYLCV and the betasatellite compared to plants only infected with TYLCV (Fig. 2.4A). The presence of the betasatellite in systemic leaves was confirmed by PCR (data not shown). For this reason, the compromising nature of betasatellites, and their encoded TGS suppressor protein, on $T y-1$ resistance was further analysed in stably transformed N. benthamiana Ty-1 plants. To this end, Ty-1 stable transformants, next to WT N. benthamiana plants, were challenged with TYLCV singly, or in a mixed setting with the betasatellite. At 19 dpi WT plants again showed yellowing and curling of the top leaves in the presence of TYLCV, whereas the transgenic Ty-1 N. benthamiana plants only exhibited very mild symptoms. In the additional presence of the betasatellite both WT and the transgenic plants exhibited more severe yellowing and curling although the symptoms in the transgenic plants infected with TYLCV and the betasatellite were less pronounced than in WT plants infected with TYLCV only (average symptom score WT+TYLCV of 2.7, Ty-1+TYLCV+betasatellite of 2, see Fig. 2.4A). When virus titers were determined by qPCR, a co-infection with the betasatellite led to a 2-fold increase of TYLCV titers in systemic leaves from $T y-1$ transgenic plants, while a reduction in virus titers was observed in WT (susceptible) plants (Fig. 2.4B). This difference was observed in all 4 transgenic lines and during two independent repetitions.

\section{Co-expression of $\beta C 1$ is sufficient to compromise $T y-1$ resistance}

To test whether the compromising effect of the betasatellite on $T y$ - 1 resistance against TYLCV was due to co-replication of the betasatellite with TYLCV or to suppression of TGS by the betasatellite encoded protein $\beta C 1$, experiments were repeated, but this time $T y$ - 1 stable transformants were challenged with TYLCV in the presence of a potato virus $X(P V X)$ vector expressing the $\beta C 1$ protein. Considering that so far only for tomato yellow leaf curl China virus associated betasatellite (TYLCCNB) $\beta C 1$ protein TGS suppression activity has been reported (Yang, Xie, et al., 

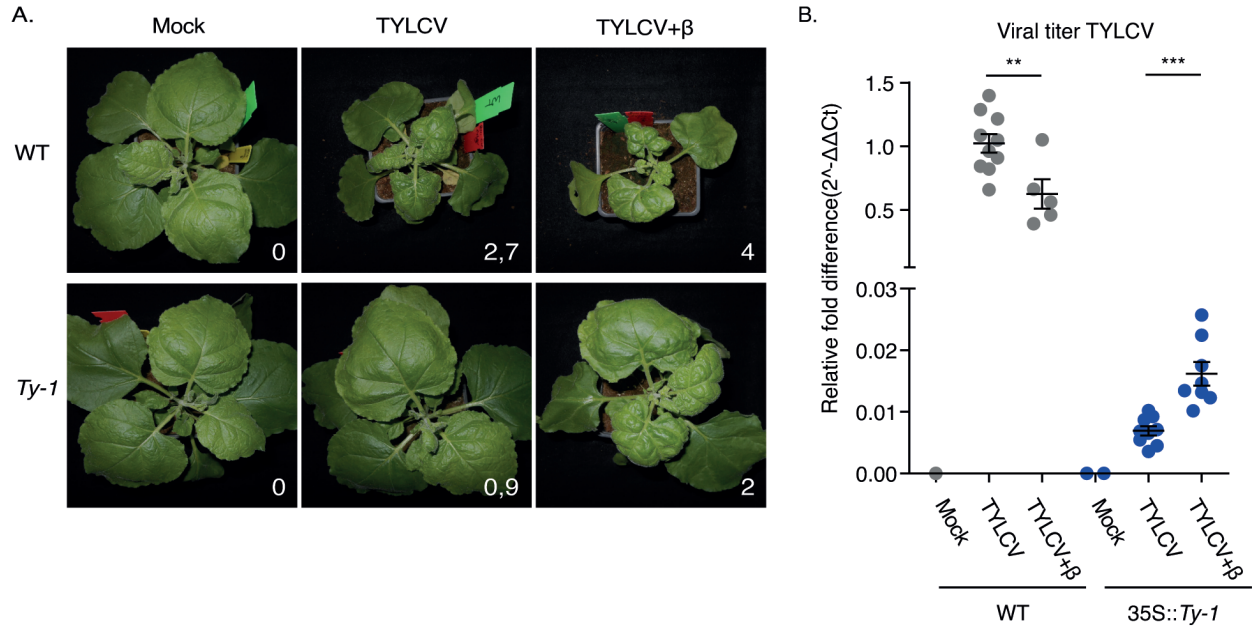

Figure 2.4: Breaking of resistance by co-replication of AYVB in Ty-1 transgenic N.benthamiana.

A. Pictures taken $19 \mathrm{dpi}$ from WT and 35S::Ty-1 plants infected with TYLCV only or co-infected with AYVB (denoted as $\beta$ in the figure). The symptom score is indicated at the bottom right corner. B. Systemic leaves of WT and 35S::Ty-1 N. benthamiana plants were harvested $19 \mathrm{dpi}$ and DNA was isolated. The viral titer was determined via qPCR relative to the presence of genomic DNA (25S rRNA) and the relative fold difference was calculated via the $2^{\wedge}$-DeltaDeltaCt method and normalized to the group WT infected with TYLCV (set to 1). Every point represents one plant. Lines represent means and standard error of the mean of biological replicates. Please note the $y$-axis is split to show the low titers. Asterisks indicate significant differences between plants infected with TYLCV only or co-infected with AYVB according to T-test analysis $\left({ }^{* *}=p<0.01\right.$; $* * *=p<0.001)$.

2011) and in the experiments here AYVB was used, TYLCCNB- $\beta C 1$ and a functionally deficient mutant (Cui et al., 2005; Yang, Xie, et al., 2011) were included as positive and negative control, respectively. Twenty-five days old WT and Ty-1 transgenic $N$. benthamiana plants were infected with TYLCV via agroinfiltration and at $10 \mathrm{dpi}$ were challenged with PVX from which either no protein, the $\beta C 1$ protein of AYVB, the $\beta C 1$ protein of TYLCCNB or the mutated TYLCCNB- $\beta C 1$ protein was expressed. The challenge with PVX was optimized at 10 days post TYLCV agroinfection, due to PVX causing systemic infections much faster than TYLCV. Eight days post PVX infection, plants were analysed for development of disease symptoms caused by TYLCV and/or PVX. Since PVX (empty vector) infections also caused chlorosis of the top leaves, these plants were more difficult to score for TYLCV symptoms. Whereas WT $N$. benthamiana plants infected with TYLCV showed symptoms as earlier observed (Fig. 2.5A), a mixed infection with PVX-TYLCCNB- $\beta C 1$ or PVX-AYVB- $\beta C 1$ 
A.
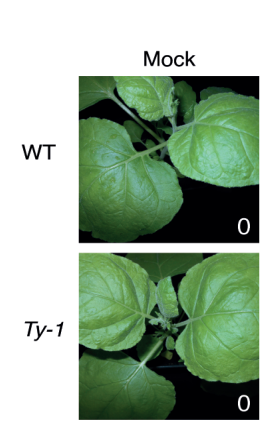

B.

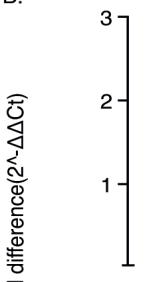

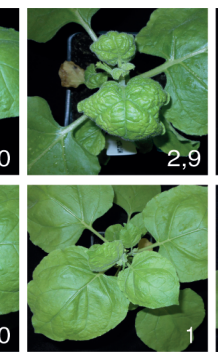

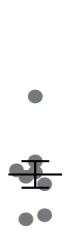

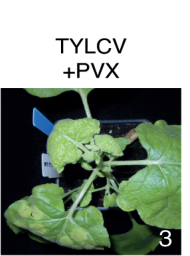

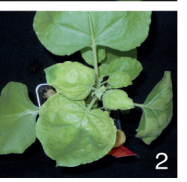

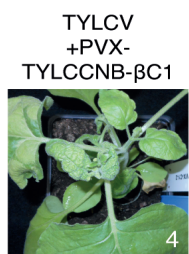

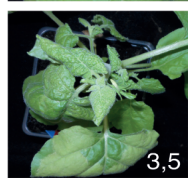

Viral titer TYLCV
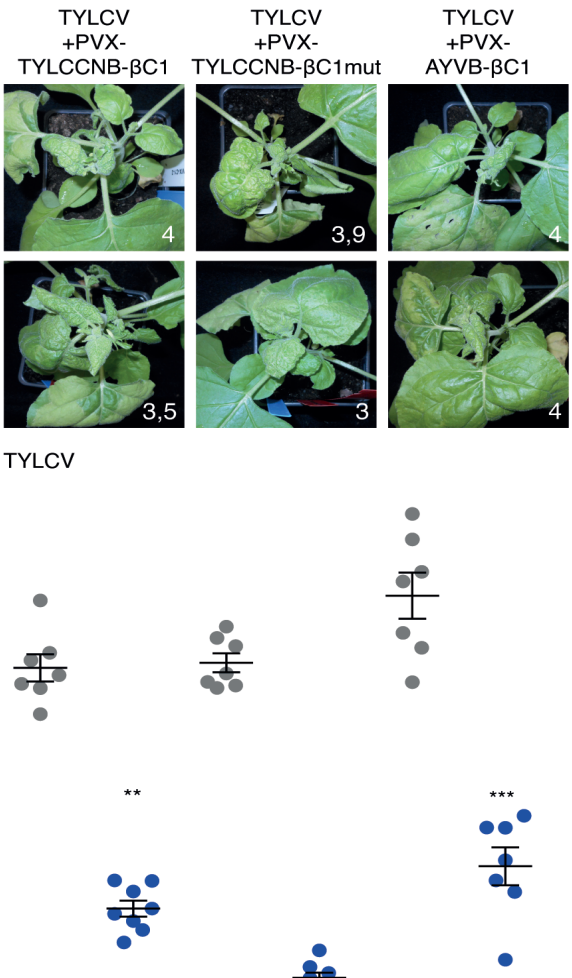

-

0.000

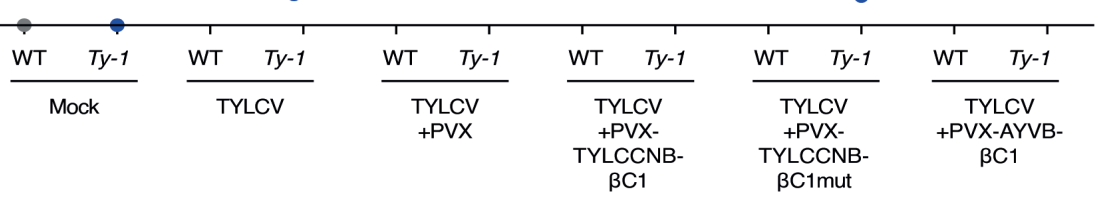

Figure 2.5: $\beta C 1$ protein expressed via a PVX vector compromises resistance against TYLCV in Ty-1 transgenic N. benthamiana.

A. WT and Ty-1 transgenic $N$. benthamiana plants were infected with TYLCV and at 10 dpi were challenged with PVX expressing either TYLCCNB- $\beta C 1$, TYLCCNB- $\beta C 1$ mutant, AYVB- $\beta C 1$ or empty PVX vector. Eight days post PVX infection, pictures were taken and plants were scored for disease symptoms, indicated in the bottom right corner. B. From the plants described under 2.5A, samples of systemic leaves were collected, DNA was isolated and the titer of TYLCV was determined via qPCR. The viral titer was normalized to the presence of genomic DNA (25S rRNA) and the fold difference was calculated according to the $2^{\wedge}$-DeltaDeltaCt method relative to WT plant infected with TYLCV only (set to 1). Every point represents one plant. Lines represent means and standard error of the mean of biological replicates. Please note the $y$-axis is split to show the low titers. Asterisks indicate significant differences according to one-way analysis of variance. In the analysis WT TYLCV only infected plants were compared with the different WT co-infected groups and the same was done for the Ty-1 transformants $(* *=p<0.01 ; * * *=p<0.001)$. 
intensified the curling and chlorosis (Fig. 2.5A) similarly as during a co-infection with the betasatellite (Fig. 2.4). During a co-infection of TYLCV and the PVX-TYLCCNB$\beta C 1$ mutant (encoding a non-functional, mutant $\beta C 1$ TGS suppressor) only some necrosis and increased chlorosis was observed compared to TYLCV only. In the stably transformed Ty-1 N. benthamiana plants, TYLCV only caused some slight chlorosis, but the additional presence of PVX-AYVB- $\beta C 1$ or PVX-TYLCCNB- $\beta C 1$, and not of PVX-TYLCCNB- $\beta C 1$ mutant, caused severe curling and chlorosis. To determine whether the increase of symptoms in the susceptible WT and Ty-1 transgenic $N$. benthamiana plants also correlated with a higher TYLCV titer, samples were collected from systemically infected leaves and DNA purified for qPCR analysis. In WT $N$. benthamiana no significant change in virus titers was observed with TYLCV only versus a mixed infection with TYLCV and one of the PVX constructs (Fig. 2.5B). In the case of a mixed infection with PVX-AYVB- $\beta C 1$ a trend of increased TYLCV titers was observed, which contrasted the reduced titers seen in the presence of AYVB. In the Ty-1 transgenic plants challenged with TYLCV, virus titers were again drastically reduced compared to WT plants, and these titers did not change by the addition of PVX-empty or PVX-TYLCCNB- $\beta C 1$ mutant. However, in the additional presence of PVXAYVB- $\beta C 1$ or PVX-TYLCCNB- $\beta C 1$ TYLCV titers increased 2.7 and 2 -fold, respectively (Fig. 2.5B). These data indicated that the presence of a betasatellite compromised $T y-1$ resistance by means of its encoded TGS suppressor, the $\beta C 1$ protein.

\section{Discussion}

Earlier we have shown that $T y$-1 confers resistance against the monopartite TYLCV (Israel strain, Almeria isolate) and the bipartite begomovirus ToSRV. We have also shown that $T y-1$ resistance involves an amplification of the antiviral RNAi response, leading to increased levels of viral siRNA production and concomitant cytosine methylation within the viral DNA genome (Butterbach et al., 2014). Here it is shown that, besides TYLCV, Ty-1 also confers resistance to TYLCSV, another species from the TYLCV cluster of begomoviruses, and to the completely distinct leafhoppertransmitted curtovirus BCTV. Furthermore, stable transformants of susceptible tomato $\mathrm{MM}$ and $\mathrm{N}$. benthamiana expressing a $T y-1$ transgene exhibited the same resistance spectrum as tomato $T y-1$ introgression lines, excluding the involvement 
of other resistance loci in this broad-spectrum resistance. In the presence of a betasatellite, but also upon trans complementation of the $\beta C 1$ protein expressed from a PVX virus vector, Ty-1 resistance against TYLCV was being compromised. Altogether, these results demonstrate that $T y-1$ provides resistance to a wide range of geminiviruses, which is not restricted to begomoviruses only, and is compromised by suppressors of RNAi that interfere at TGS.

Whereas this study shows for the first time that $T y-1$ also hampers the replication of a curtovirus, the findings on the effectiveness of $T y-1$ against a group of TYLCV-like viruses are supported and in agreement with the outcome of other studies. Others have reported on the efficacy of Ty-1 against the monopartite TYLCV-MId (Shahid et al., 2013), TYLCSV (Barbieri et al., 2010), tomato curly stunt virus (ToCSV), tomato leaf curl Bangalore virus (ToLCBV), honeysuckle yellow vein mosaic virus (HYVMV) and tobacco leaf curl Japan virus (TbLCJV) (Pietersen and Smith, 2002; Prasanna et al., 2015; Shahid et al., 2013) and to multiple bipartite begomoviruses including tomato mottle virus (ToMoV), tomato leaf curl New Delhi virus (ToLCNDV) and tomato leaf curl Palampur virus (ToLCPalV) (Prasanna et al., 2015). In our earlier study, Ty-1 was shown to confer resistance to the bipartite begomovirus ToSRV as well (Butterbach et al., 2014).

Ty-1 does not confer resistance to RNA viruses, as recently shown for tomato spotted wilt virus (TSWV) and CMV (Butterbach et al., 2014). Also, in the study presented here, the symptoms caused by a PVX infection were not different between WT and stably transformed Ty-1 N. benthamiana. However, RNA viruses may compromise $T y-1$ resistance against geminiviruses, as earlier shown during a co-infection of TYLCV and CMV. The observed increase in TYLCV titers in the latter case was explained to be due to inhibition of the TGS response by the CMV $2 \mathrm{~b}$ RNAi suppressor protein (Butterbach et al., 2014; González et al., 2010; Hamera et al., 2012). This idea is strengthened by the current study, showing a compromising effect on $T y-1$ resistance by AYVB, and also solely by the AYVB $\beta C 1$ and TYLCCNB $\beta C 1$ proteins expressed from a PVX virus vector. TYLCCNB $\beta C 1$ protein has earlier been demonstrated to suppress TGS via inhibition of the enzyme S-adenosyl homocysteine hydrolase (SAHH), an enzyme that is required for production of the methyl donor used in the TGS pathway (Yang, Xie, et al., 2011). CMV 2b in contrast has been 
shown to hamper TGS via inhibition of AGO4 (González et al., 2010; Hamera et al., 2012). Despite the fact that these viral suppressors act differently, the ability to inhibit TGS at (any) different step(s) of the pathway apparently is sufficient to compromise Ty-1-mediated resistance.

Recently, Conflon et al. (2018) (Conflon et al., 2018) also reported on the compromising effect of a co-replicating betasatellite on Ty-1 resistance. Ty-1 tomato lines co-infected with different TYLCV strains and a cotton leaf curl Gezira betasatellite revealed an increase of disease symptoms, but interestingly titers only increased in the mixed infection case with TYLCV-II, while those of TYLCV-MId were decreased upon addition of the betasatellite. How to explain these effects caused by one and the same betasatellite remains to be further investigated, although it is likely that this has to do with the virus strains and not the betasatellite. Considering that the efficacy of $T y-1$ is compromised by trans complementing TGS suppressors, also the suppressors encoded by the geminiviral DNA genome of TYLCV-II and TYLCV-Mld will affect Ty-1 resistance. If their suppressors of RNAi differ in strength, $T y-1$ mediated TGS will be suppressed to varying degrees, leading to different levels of reduction in virus titers. Although speculative, whether this also explains the observed difference in efficacy of Ty-1 resistance towards TYLCV and BCTV remains to be analysed. To this end, initial attempts were performed to detect TGS suppression in 16C-TGS transgenic plants (as shown by Buchmann et al., 2009). Therefore, 16C-TGS plants were challenged with, among others, BCTV and TYLCV. PVX-TYLCCNB- $\beta C 1$ was shown to reverse the transcriptionally silenced status of the GFP transgene and therefore was included as positive control (Yang, Xie, et al., 2011). RNA was isolated from systemically infected leaves to quantify the GFP mRNA expression via RT-qPCR. No difference in GFP expression was measured between mock infected plants and any of the infected plants (Sup. Fig. 2.1), indicating that, unexpectedly no suppression of TGS had occurred. During repeated attempts no GFP expression was recovered from the 16C-TGS lines, even with the positive control. So, the experiment failed and remain to be repeated (Buchmann et al., 2009).

Recently several articles have been published on $T y-1$ resistance breaking strains of TYLCV in cultivations of Ty-1 bearing tomato. Samples of $T y-1$ plants showing 
TYLCV-like symptoms collected in Morocco, Italy and Spain revealed the presence of viruses derived from a recombination event between TYLCV and TYLCSV, in which a noncoding region between the origin of replication and the start of the coding region of V2 were exchanged (Belabess et al., 2015; Granier et al., 2019; Panno et al., 2018; Torre et al., 2019). In Morocco, this recombinant replaced both parental strains, but also under lab conditions this recombinant was positively selected in $T y-1$ bearing plants (Belabess et al., 2015; Belabess et al., 2016). So far, the mechanism behind the improved fitness of this resistance breaking strain is unknown, but the region of recombination covers the promoter region of the coat protein. The resistance breaking, therefore, might be due to (a combination of) changes in replication efficiency or viral gene expression, or the region of recombination might be less prone to TGS.

In conclusion, $T y-1$ is a unique resistance gene that confers resistance to a broad spectrum of geminiviruses, as demonstrated for begomoviruses and a curtovirus. Although $T y-1$ is likely to confer resistance to all geminiviruses, suppression of RNAi by co-replicating betasatellites, or other RNA viruses, but also by suppressors encoded from the geminiviral DNA genome itself, seems to present the Achilles' heel of the resistance mechanism. Whether $T y-1$ resistance can also be compromised by an infection involving a bacterial or fungal/oomycete pathogen, some of which are shown to either cause hypomethylation or suppress (the amplification of) RNAi (Hou et al., 2019; Navarro et al., 2008; Pavet et al., 2006), remains an interesting question. The effect of co-infections on $T y-1$ resistance is important knowledge in light of disease management strategies, and simultaneously underlines the importance for monitoring the presence of other pathogens that encode suppressors of RNAi and interfere at the level of TGS, during cultivation of $T y-1$ bearing tomato plants. 


\section{Experimental Procedures}

\section{Plant material and virus sources}

Throughout this study S. lycopersicum and $N$. benthamiana plants were maintained under greenhouse conditions at $23{ }^{\circ} \mathrm{C}$ during the day and at $21^{\circ} \mathrm{C}$ at night $(16 \mathrm{~h}$ light $/ 8 \mathrm{~h}$ dark regime) and a relative humidity of $60 \%$. S. lycopersicum cv. MM was used as susceptible control and a Ty-1 introgression line was derived from S. chilense LA1969 (Verlaan et al., 2013). 16C-TGS N.benthamiana plants were kindly provided by dr. D. Bisaro (The Ohio State University, Columbus, Ohio ) (Buchmann et al., 2009). Plants were infected with geminiviruses by means of agroinoculation of infectious clones. Agroinfectious clones used in this study were from two isolates of TYLCV, namely the Israel strain isolated from Almeria, Spain, as described by Morilla et al. (2005) (GenBank AJ489258.1), and the Israel strain isolated from Shanghai, China, named TYLCV-[CN:SH2] (GenBank AM282874, (Zhang et al., 2009)), an isolate of TYLCSV (GenBank X61153.1 (Noris et al., 1998)) and an isolate of BCTV California Logan (GenBank M24597.2 (Stanley et al., 1986)). Co-infections were performed with an agroinfectious clone of AYVB (GenBank AJ252072.1, (Saunders et al., 2000)).

\section{Transformation of tomato and $\mathbf{N}$. benthamiana plants with Ty-1}

To generate a construct for transformation purposes, the $T y$ - 1 full length coding sequence was amplified from complementary DNA (cDNA) of Ty-1 introgression lines with forward primer Ty-1-CDS-F and reverse primer Ty-1-CDS-R (for primer sequences see Sup. Table 2.1) (Verlaan et al., 2013). PCR products were cloned into the pENTR/D-TOPO vector (Invitrogen) and verified by sequence analysis, prior to recombination into pK7WG2 via an LR reaction (Invitrogen). The resulting plasmid was transferred to Agrobacterium tumefaciens (A. tumefaciens) strain AGL1 by electroporation. Transformation of cotyledons from S. lycopersicum MM and N. benthamiana was carried out according to the method described by Appiano et al. (2015). Plants regenerated from kanamycin-resistant primary transformants $\left(T_{1}\right)$ were selected and self-pollinated to produce $T_{2}$ families. From each segregating $T_{2}$ family transgenic lines were selected by PCR amplification of the NPTII gene 
cassette (using primers NPT3 and NPT4 (Heilersig et al., 2006), amplifying a 722-bp fragment) and a partial $T y-1$ gene sequence (using primers CaMV 355 promoter primer 35S-F and gene insert-specific primer Ty-1-R, amplifying a 822-bp fragment).

\section{PVX constructs}

PVX infectious clones encoding the betasatellite C1 protein gene were constructed as follows: The open reading frame of AYVB C1 was amplified from the infectious clone (GenBank AJ252072.1) using primers AYVB-C1-F and AYVB-C1-R (Sup. Table 2.1). Sequences for $C 1$ and a $C 1$ mutant (Cui et al., 2005) of the TYLCCNB were ordered as a gene block from Integrated DNA Technologies (IDT, Belgium) and were based on GenBank accession AJ421621.1. The gene blocks were cloned into pJet1.2 according to the manufacturers' protocol (Thermofisher), and their sequences verified. Next, the TYLCCNB C1 gene was amplified with primers TYLCCNB-C1-F and TYLCCNB-C1-R containing a 5'- Clal or Sall restriction site. Amplicons were purified and subsequently digested with Sall and Clal, prior to cloning into the Sall and Claldigested PVX vector pGR107 (Jones et al., 1999). Positive clones were selected and verified by sequence analysis. The agrobacterium strain GV3101, containing the helper plasmid pSoup, was transformed with the constructs PVX-AYVB- $\beta C 1$, PVX-TYLCCNB- $\beta C 1$, PVX-TYLCCNB- $\beta C 1$ mut or with the empty pGR107 plasmid, for subsequent agroinfiltration of plants.

\section{Agrobacterium Transient Transformation assay}

Agrobacterium transient transformation assays were performed following a slightly modified protocol of Bucher et al. (2003). In brief, A. tumefaciens was grown overnight at $28{ }^{\circ} \mathrm{C}$ in $3 \mathrm{ml}$ LB3 (10 gL-1 trypton, 5 gL-1 yeast, 4 gL-1 NaCl, $1 \mathrm{gL}-1 \mathrm{KCl}, 3 \mathrm{gL}-1 \mathrm{MgSO} 4.2 \mathrm{H} 2 \mathrm{O})$ medium containing proper antibiotic selection pressure. From this culture, $600 \mu \mathrm{l}$ was transferred and incubated overnight in 3 $\mathrm{ml}$ induction medium (10.5 gL-1 $\mathrm{K}_{2} \mathrm{HPO}_{4}, 4.5 \mathrm{gL}-1 \mathrm{KH}_{2} \mathrm{PO}_{4}, 1 \mathrm{gL}-1\left(\mathrm{NH}_{4}\right) 2 \mathrm{SO}_{4}, 0.5 \mathrm{gL}-1$ sodium citrate. $2 \mathrm{H}_{2} \mathrm{O}, 1 \mathrm{mM} \mathrm{MgSO} \mathrm{m}_{4} .7 \mathrm{H}_{2} \mathrm{O}, 0,2 \%(\mathrm{w} / \mathrm{v})$ glucose, 0,5\% (v/v) glycerol, $50 \mu \mathrm{M}$ acetosyringone, $10 \mathrm{mM}$ 2-(N-Morpholino)ethanesulfonic acid ((MES), pH 
5.6)). The next day bacteria were pelleted by centrifugation ( $15 \mathrm{~min} .2670 \mathrm{~g}$ ) and resuspended in MS MES buffer (Murashige and Skoog medium (Duchefa biochemie) supplemented with $150 \mu \mathrm{M}$ acetosyringone, $10 \mathrm{mM}$ MES and $87 \mathrm{mM}$ sucrose) at an OD600 of 0.5 per construct. One hour after plants were watered in excess, the first two true leaves were fully infiltrated with this mixture by pressure inoculation with a needle-less syringe on the abaxial side of the leaf.

\section{Disease Assessment}

Plant responses were scored several times during the whole disease assay on viral symptom development. The time span of the disease assay varied per experiment and lasted up to 50 days. Depending on the lines and experiments, five to ten plants were challenged. Each plant was rated using a 0 to 4 disease severity index (DSI) described by Friedmann et al. (1998), where 0 indicates no viral disease symptoms, and 4 means severe symptoms. Intermediate scores, $0.5,1.5,2.5$, and 3.5 were incorporated for more precise scoring.

\section{Nucleic acid purification}

Systemically infected leaves were harvested and snap frozen in liquid nitrogen. The samples were grinded with either mortar and pestle or by using precellys (Bertin Instruments, France) for $10 \mathrm{sec}$ at $5000 \mathrm{rpm}$. To determine geminivirus titers, DNA was isolated following the cetyltrimethyl ammonium bromide (CTAB) method of Doyle and Doyle (1987) with slight modifications as described by Fulton et al. (1995). In brief, grinded plant material was incubated for 1 hour at $65^{\circ} \mathrm{C}$ with CTAB buffer (0.1 M TRIS, 0.7 M NaCl, 0.01 M EDTA, 2\% CTAB). After chloroform isoamyl alcohol extraction and spinning of the samples, the upper aqueous phase was transferred to a new tube and the DNA precipitated from the suspension by adding isopropanol in a 1:1 ratio. DNA was pelleted by centrifugation for $10 \mathrm{~min}$ at $10 \mathrm{krpm}$, dried and dissolved in milliQ. For Ty-1 expression analysis, RNA from $N$. benthamiana plants was extracted using TRIZOL according to the manufacturers' protocol (Invitrogen) and RNA from tomato plants by using the RNeasy Plant Mini Kit (Qiagen) following 
the manufacturer's protocol. DNA and RNA concentrations were measured with a Nanodrop ND-1000 device.

\section{Detection of the betasatellite}

DNA of AYVB was amplified using AYVB specific primers AYVB-F1, AYVB-F2 and AYVB-R (Sup. Table 2.1). The combination of AYVB-F1 and AYVB-R resulted in a product of $823 \mathrm{bp}$, whereas AYVB-F2 and AYVB-R resulted in a product of $457 \mathrm{bp}$. The PCR was performed with GoTaq polymerase according to the manufacturers' protocol (Promega), using the following cycling conditions: 5 min $95^{\circ} \mathrm{C}$; 40 cycles of $30 \sec 95^{\circ} \mathrm{C}, 30 \mathrm{sec} 55^{\circ} \mathrm{C}, 60 \mathrm{sec} 72{ }^{\circ} \mathrm{C} ; 7 \mathrm{~min} 72{ }^{\circ} \mathrm{C}$. Amplified PCR products were visualized in a $1 \%$ agarose gel using ethidium bromide.

\section{Virus titration}

Relative virus titers of the TYLCV Almeria isolate, TYLCSV, TYLCV-[CN:SH2] and BCTV were determined by qPCR using 25S rRNA ( $N$. benthamiana) or EF1 $\alpha$ (S. lycopersicum, Solyc06g005060) as internal control. To measure samples of $N$. benthamiana, the reaction mixture contained 1x SybrSelect (Applied Biosystems), $375 \mathrm{nM}$ forward primer, $375 \mathrm{nM}$ reverse primer and $10 \mathrm{ng}$ genomic DNA, while for S. lycopersicum samples the iQ SYBR Green supermix (Bio-Rad) was used. For PCR amplification the following primers were used (Sup. Table 2.1): for TYLCV Almeria isolate primers TYLCV-Alm-qPCR-F and TYLCV-Alm-qPCR-R (Powell et al., 2012), for TYLCSV primers TYLCSV-qPCR-F and TYLCSV-qPCR-R, for TYLCV-[CN:SH2] primers TYLCV-CN-qPCR-F and TYLCV-CN-qPCR-R, for BCTV either primers BCTV-qPCR-F1 and BCTV-qPCR-R1 or BCTV-qPCR-F2 and BCTV-qPCR-R2. During $q P C R$ 25S rRNA was amplified using primers 25S-qPCR-F and 25S-qPCR-R, and EF1 $\alpha$, using primers SIEF1a-qPCR-F and SIEF1a-qPCR-R.

The qPCR was performed in a Bio-Rad CFX384, using the following cycling conditions: 2 min $95^{\circ} \mathrm{C}, 40$ cycles of $15 \sec 95^{\circ} \mathrm{C}$ and 1 min $60^{\circ} \mathrm{C}$, followed by a melting curve with $0.5^{\circ} \mathrm{C}$ steps from $60{ }^{\circ} \mathrm{C}$ to $95^{\circ} \mathrm{C}$ to determine PCR specificity. Relative viral 
titers were calculated using the DeltaDeltaCt method (Livak and Schmittgen, 2001). Values were normalized relative to the internal control 25S rRNA or EF1 $\alpha$, and calibrated to levels of the control plants, which were set as 1 .

\section{Ty-1 gene expression analysis}

Prior to gene expression analysis $1 \mu \mathrm{g}$ of purified total RNA of $N$. benthamiana samples was treated with TURBO DNAse (Invitrogen) following the manufacturers' instructions. First strand cDNA was synthesized using random hexamers (Roche) and M-MLV reverse transcriptase according to the manufacturers' protocol (Promega). The RT-qPCR was performed in a total volume of $10 \mu \mathrm{l}$, containing $1 \mu \mathrm{l}$ of $5 \mathrm{x}$ diluted cDNA, 1x SybrSelect (Applied biosystems), $375 \mathrm{nM}$ forward primer and $375 \mathrm{nM}$ reverse primer. Total RNA of S. Iycopersicum was treated with DNase I, Amplification Grade (Invitrogen) following the manufacturers' instructions. CDNA was synthesized using the iScript cDNA Synthesis Kit (Bio-Rad) and the RT-qPCR performed with the iQ SYBR Green supermix (Bio-Rad). To determine Ty-1 expression levels, either Ty-1qPCR-F1 and Ty-1-qPCR-R1 or Ty-1-qPCR-F2 and Ty-1-qPCR-R2 primers were used. The gene expression was measured relatively to the housekeeping gene EF1 $\alpha$ by using the primers nbEF1 $\alpha-q P C R-F$ and nbEF1 $\alpha-q P C R-R$ in case of $N$. benthamiana samples or SIEF1a-qPCR-F and SIEF1a-qPCR-R in case of S. lycopersicum samples (Sup. Table 2.1). The RT-qPCR was performed in a Bio-Rad CFX384, using the same conditions as for the virus titration. Relative gene expression was calculated using the formula $2^{\wedge}$-(DeltaCt Ty-1 (Ct Ty-1 - Ct EF1 $\alpha$ ) - DeltaCt MM (Ct Ty-1 - Ct EF1 $\alpha$ )) (Livak and Schmittgen, 2001).

\section{TGS suppression essay}

16C-TGS plants were seeded in an insect free greenhouse and after 17 days seedlings were screened for green fluorescent protein (GFP) expression using a handheld UV light. Plants negative for GFP expression were selected and transplanted to pots with a diameter of $16.5 \mathrm{~cm}$. Three weeks post seeding they were challenged by agroinoculation with an infectious clone. Systemic leaves where monitored for the 
appearance of GFP expression 17 days post infection (dpi) using a handheld UV light and samples of systemic leaves were taken for RNA extraction. RNA was extracted and GFP mRNA levels were measured relatively to the housekeeping gene EF1 $\alpha$ via qPCR as described previously. For detection of GFP, primers GFP-qPCR-F and GFPqPCR-R were used.

\section{Acknowledgements}

The authors would like to thank Prof. Eduardo Rodríguez Bejarano (Universidad de Málaga, Malaga, Spain) for providing the infectious TYLCV (Almeria isolate) clone, dr. Emanuela Noris (Institute of Plant virology (IVV), Torino, Italy) for providing the infectious clone of TYLCSV and dr. Keith Saunders (John Innes Centre, Norwich, UK) for providing the infectious clone of BCTV and AYVB. The authors would like to thank Annelies E. H. M. Loonen for making the primary tomato and N. benthamiana transformants. Monique van Oers (Wageningen University) is acknowledged for critically reading the chapter. This research was financially supported by NWO-CNPq within the Joint Research Project Biobased Economy (729.004.011), Plant Breeding of Wageningen University \& Research and a vegetable breeding company. The authors declare no conflicts of interest. 


\section{Supplemental data}

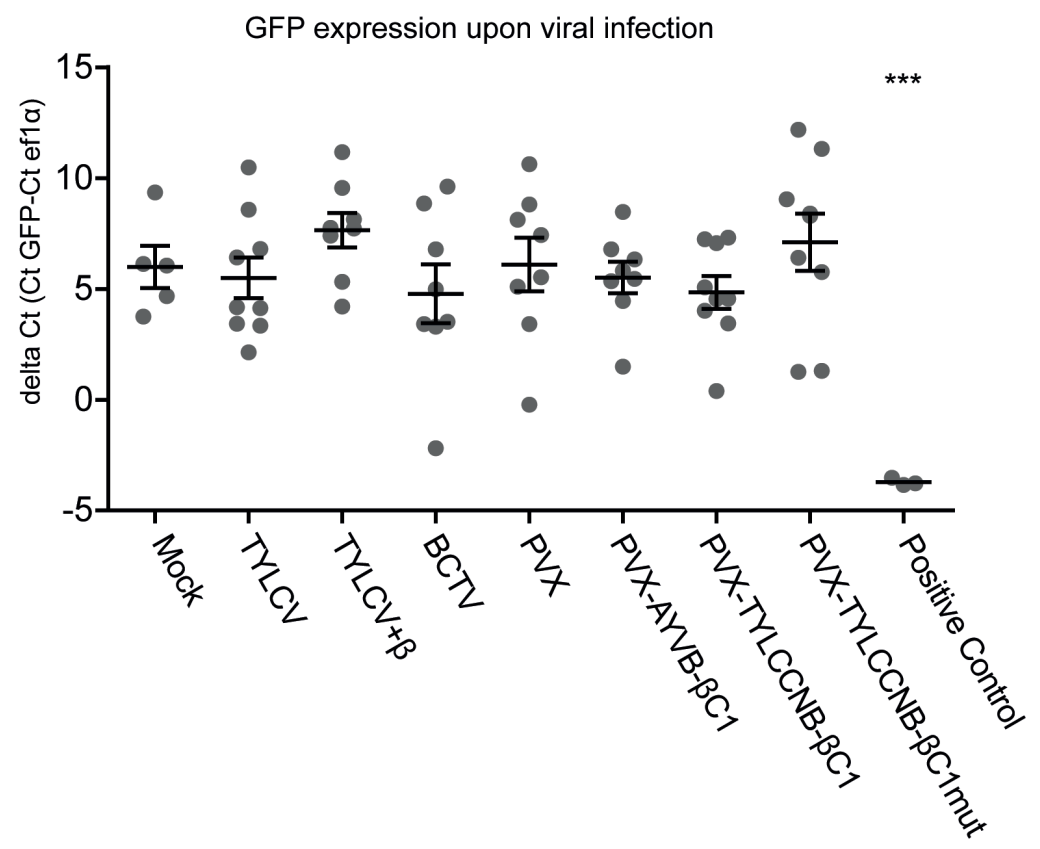

Supplemental Figure 2.1: TGS suppression activity of geminiviruses TYLCV and BCTV and $\beta C 1$ protein.

16C-TGS plants were infected with either TYLCV only, TYLCV and AYVB, BCTV, empty PVX or PVX encoding for TYLCCNB- $\beta C 1$, TYLCCNB- $\beta C 1$ mutant or AYVB- $\beta C 1$ respectively. Positive control are plants which showed spontaneous expression of GFP after germination, screened with an UV light. $17 \mathrm{dpi}$ systemic leaves were collected for RNA purification and the expression level of GFP was determined by RT-qPCR relative to the housekeeping gene EF1 $\alpha$. The DeltaCt is depicted. Every point represents one plant, lines represent means and standard error of the mean of biological replicates. Asterisks indicate significant differences between treated plants and plants receiving a mock treatment according to one-way analysis of variance $\left({ }^{* * *}=p<0.001\right)$. 
Supplemental Table 2.1: List of sequences of primers used in this research.

\begin{tabular}{|c|c|}
\hline Name & Sequence $5^{\prime}-3^{\prime}$ \\
\hline $25 s-q P C R-F$ & ATAACCGCATCAGGTCTCCA \\
\hline $25 s-q P C R-R$ & CCGAAGTTACGGATCCATTT \\
\hline $35 S-F$ & GCTCCTACAAATGCCATCA \\
\hline AYVB-C1-F* & CCATCGATATGACTATATCATATACCAACG \\
\hline AYVB-C1-R* & GCGTCGACTTATACGGTTACATTCTTGTATAC \\
\hline AYVB-F1 & AGTGCTGGTGACCTTGTTGAA \\
\hline AYVB-F2 & TTCAGAAAAAATGGGAGCGCAGCG \\
\hline AYVB-R & CGTGTCGCGAATTGATGCCG \\
\hline BCTV-qPCR-F1 & GGATGGAAATGTGCTGACCT \\
\hline BCTV-qPCR-F2 & GTTGGGTGCTGGTGGTATAG \\
\hline BCTV-qPCR-R1 & CTACACGAAGATGGGCAACC \\
\hline BCTV-qPCR-R2 & CGTTCTTCGCTCTCTGACTATC \\
\hline GFP-qPCR-F & CCTGTCCTTTTAACCAGACAACCA \\
\hline GFP-qPCR-R & CCCAGCAGCTGTTACAAACTCA \\
\hline nbEF1 $\alpha-q P C R-F$ & AGCTTTACCTCCCAAGTCATC \\
\hline nbEF1 $\alpha-q P C R-R$ & AGAACGCCTGTCAATCTTGG \\
\hline NPT3 & TCGGCTATGACTGGGCACAACAGA \\
\hline NPT4 & AAGAAGGCGATAGAAGGCGATGCG \\
\hline SIEF1a-qPCR-F & ATTGGAAACGGATATGCCCCT \\
\hline SIEF1a-qPCR-R & TCCTTACCTGAACGCCTGTCA \\
\hline Ty-1-CDS-F & CACCTTCAAGTATATACAGGAAAAATGGGTGATCCG \\
\hline Ty-1-CDS-R & CTAGAGTATTTCCTGCAAAACCGATG \\
\hline Ty-1-qPCR-F1 & CTGGGCGTGTTTTGGTCTAC \\
\hline Ty-1-qPCR-F2 & GGCAAAATATGCAGCCAGGCTTTCC \\
\hline Ty-1-qPCR-R1 & CTCAGTAGCAGCTGACCTCG \\
\hline Ty-1-qPCR-R2 & TCAGTATGTATACGAGGTTCGCCGT \\
\hline Ty-1-R & CTGAGGGCTTGCACAGGCCAAT \\
\hline TYLCCNB-C1-F* & CCATCGATATGTATCATCCACAACAAATAAACATG \\
\hline TYLCCNB-C1-R* & GCGTCGACTCATACATCTGAATTTGTAAATACATCATAC \\
\hline TYLCSV-qPCR-F & ATGCTACGGTTGTTGGAGGT \\
\hline TYLCSV-qPCR-R & TCGCCTGCTCTTGATGATTA \\
\hline TYLCV-Alm-qPCR-F & TTCGTCTAGATATTCCCTATATGAGGAGGTA \\
\hline TYLCV-Alm-qPCR-R & GGGAAGCCCATTCAAATTAAAGG \\
\hline TYLCV-CN-qPCR-F & GCTCGTAGAGGGTGACGAAG \\
\hline TYLCV-CN-qPCR-R & TCTGCAATCCAGGACCTACC \\
\hline
\end{tabular}




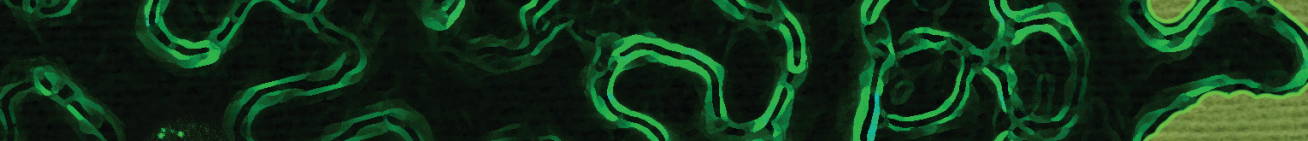

$5 \cdot C 254$

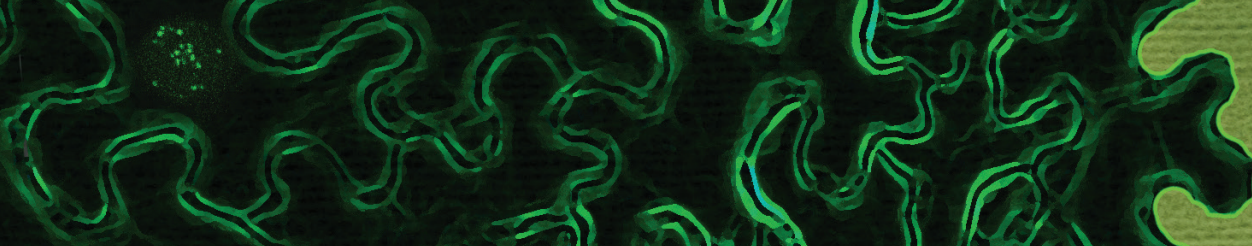

$\left.n^{2} s^{3}\right\}$ 3र $2,53 \sqrt{2}$

रुज (5)

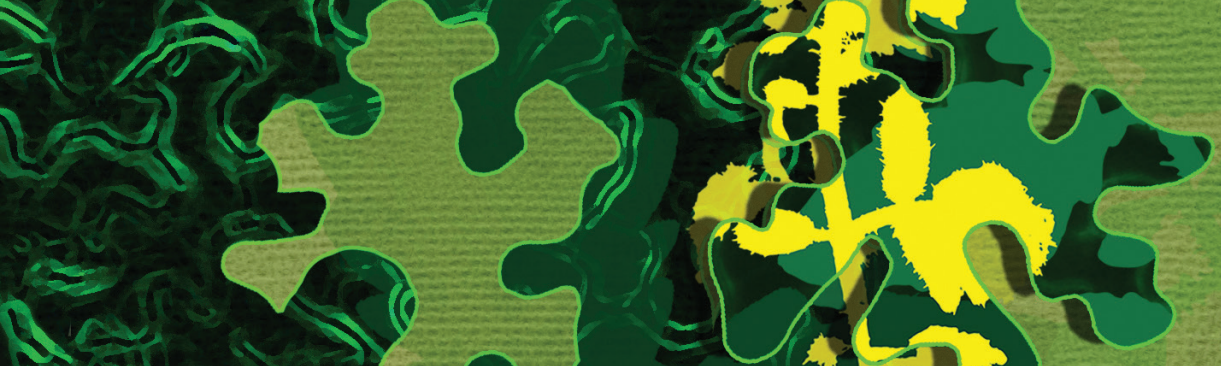

की

ว 2 .

(2)

$\lim _{2}\left(\sin _{n}\right)$

$80^{m}$

$(2 \sqrt{2}$

S)

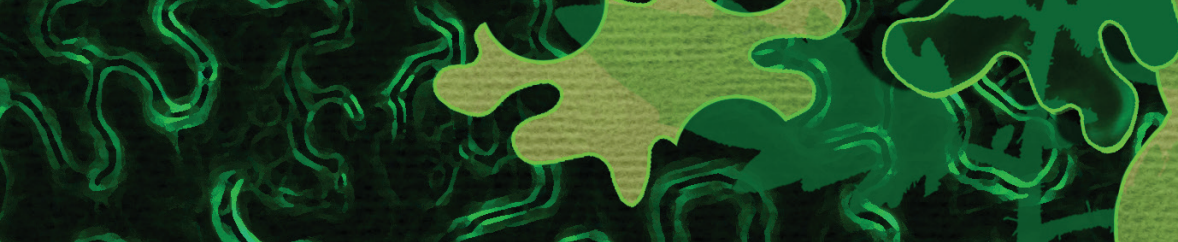




\section{Chapter 3}

Small RNA profiling of susceptible and resistant $T y-1$ encoding tomato plants upon TYLCV infection

C.M. Voorburg, Y. Bai, R. Kormelink 


\section{Summary}

Ty-1 presents an atypical dominant resistance gene that codes for an RNA-dependent RNA polymerase (RDR) of the gamma class and confers resistance to Tomato yellow leaf curl virus (TYLCV) and other geminiviruses. Tomato lines bearing $T y-1$ not only produce relatively higher amounts of viral small interfering (vsi)RNAs but viral DNA also exhibits a higher amount of cytosine methylation. Whether Ty-1 specifically enhances post transcriptional gene silencing (PTGS), leading to a degradation of RNA target molecules and primarily relying on 21-22 nucleotides (nts) siRNAs, and/or transcriptional gene silencing (TGS), leading to methylation of cytosines within DNA target sequences and relying on 24-nt siRNAs, was unknown. In this study, small RNAs were isolated from systemically TYLCV-infected leaves of Ty-1 encoding tomato plants and susceptible tomato Moneymaker (MM) and sequence analysed. While in susceptible tomato plants vsiRNAs of the 21-nt size class were pre-dominant, their amount was drastically reduced in tomato containing Ty-1. The latter, instead, revealed elevated levels of vsiRNAs of the 22- and 24-nt size classes. In addition, the genomic distribution profiles of the vsiRNAs were changed in Ty-1 plants compared to those from susceptible MM. In MM three clear hotspots were seen, but these were less pronounced in Ty-1 plants, likely due to enhanced transitive silencing to neighbouring viral genomic sequences. The largest increase in the amount of vsiRNAs was observed in the Intergenic Region and the V1 viral gene. The results suggest that Ty-1 enhances an antiviral TGS response. Whether the elevated levels of 22-nt vsiRNAs contribute to an enhanced PTGS response or an additional TGS response involving a non-canonical pathway of RNA dependent DNA methylation will be discussed. 


\section{Introduction}

RNA interference (RNAi) is a highly conserved mechanism in eukaryotes involved in several processes like gene regulation, development and silencing of transposable elements (Ding and Voinnet, 2007; Erdmann and Picard, 2020; Han et al., 2004). It also acts as an important defence mechanism against viruses in plants. The trigger for RNAi is double stranded RNA (dsRNA), which during a viral infection can arise as e.g. replication intermediates, overlapping transcripts, or folded mRNA structures (Ding and Voinnet, 2007). The dsRNA is recognized by DICER-LIKE proteins (DCL), RNAse III-type enzymes, that cleave the dsRNA into small interfering RNA (siRNAs) of different size classes, ranging from 21-24 nucleotide (nt) in length (Hammond, 2005). From a 21-nt siRNA, one strand is loaded into a RNA-induced silencing complex (RISC) containing an Argonaute (AGO)1 core protein, which subsequently guides the search for complementary target RNA molecules. The latter either become cleaved by the slicer activity of AGO1 or become translational arrested (Mallory and Vaucheret, 2010), a pathway that is generally referred to as Post Transcriptional Gene silencing (PTGS). In contrast, one strand of a 24-nt siRNA loads into a RISC complex containing AGO4 as core component, which assists in the search for complementary target DNA sequences via a RNA scaffold. This leads to methylation of cytosine residues within the target DNA sequence via recruitment of DNA methyltransferases (Duan et al., 2015; Law and Jacobsen, 2010; Mallory and Vaucheret, 2010), and this pathway is generally named Transcriptional Gene silencing (TGS). RNA viruses are targeted by the PTGS pathway only, while DNA viruses are prone to both PTGS and TGS.

The model plant Arabidopsis thaliana encodes four DCL and ten AGO proteins (Bologna and Voinnet, 2014). The proteins act in different pathways and have specialized functions but are also found to function redundant. DCL1 is involved in the production of microRNAs (miRNAs) from (host-encoded) miRNA precursors, which regulate the expression of genes (Han et al., 2004; Zeng et al., 2003). DCL2 and DCL4 produce siRNAs of 22-nt and 21-nt in size respectively, which both function in the PTGS pathway, while DCL3 produces 24-nt siRNAs that are involved in TGS (Bologna and Voinnet, 2014). The canonical pathway leading to RNA dependent DNA methylation ( $R d D M)$ is mostly studied in the silencing of transposable 
elements, but less in the context of antiviral defence against DNA viruses (Erdmann and Picard, 2020; Kenchanmane Raju et al., 2019; Zhang et al., 2018). Recently, alternative, non-canonical, pathways leading to RdDM are found which make use of elements of the PTGS machinery, and one pathway which is DCL independent and produces siRNAs via the action of 3' to 5' exonuclease (Cuerda-Gil and Slotkin, 2016; Erdmann and Picard, 2020; Kenchanmane Raju et al., 2019; Marí-Ordóñez et al., 2013; McCue et al., 2015; Ye et al., 2016).

In plants, the RNAi signal is amplified by RNA dependent RNA polymerases (RDRs) (Wang et al., 2010; Yu et al., 2003). Arabidopsis thaliana codes for six RDRs, named RDR1 to RDR6. RDR1, 2 and 6 are the best characterised with an established role in RNAi (Willmann et al., 2011), and belong to a so-called alpha-class due to the presence of a highly conserved core motif. RDR6 converts aberrant RNA, resulting from DCL cleavage of a primary target RNA molecule or transgene expression, in dsRNA. The subsequent processing by DCLs leads to the production of a pool of secondary siRNAs (Wang et al. , 2010) that contributes to a strong antiviral response. Plants in which RDR6 has been knocked out generally become hypersusceptible to RNA viruses (Donaire et al., 2008; Schwach et al., 2005). RDR activity also leads to spreading of the silencing signal into sequences neighbouring the initially targeted sequence, a process called transitivity (de Felippes and Waterhouse, 2020; Parent et al., 2015). Like RDR6, RDR1 enhances the antiviral PTGS pathway, but it's function is less understood (Donaire et al., 2008; Garcia-Ruiz et al., 2010; Qi et al., 2009). RDR2 plays an important role in the TGS pathway and has been most well studied in relation to the silencing of transposable elements. It produces dsRNA of RNA polymerase IV transcripts, which are subsequently processed by DCL3 into 24-nt siRNAs (Blevins et al., 2015). RDR1 and RDR6 have also been implicated in a non-canonical RdDM pathway, since mutation of those RDRs results in decreased DNA methylation (Pontier et al., 2012; Stroud et al., 2013).

Tomato yellow leaf curl virus (TYLCV) is a member of the Geminiviridae family, and representative of the whitefly Bemisia tabaci-transmitted begomoviruses. TYLCV contains a circular single stranded DNA genome of $\sim 2.7 \mathrm{~kb}$ that replicates in the nucleus and codes for six proteins, two in virion sense strand and four in the complementary sense strand (Zerbini et al., 2017). Between the sense and complimentary sense 
open reading frames (ORFs), the intergenic region (IR) is located which is necessary for viral replication and functions as promoter for transcription of viral genes (Borah et al., 2016). TYLCV is prone to antiviral PTGS and TGS, and during an infection, 21nt, 22-nt and 24-nt viral siRNAs (vsiRNAs) complementary to the viral genome are produced (Piedra-Aguilera et al., 2019). The initial dsRNA trigger is thought to be secondary folding structures in mRNA and/or overlapping transcripts of the sense and complimentary sense ORFs (Ramesh et al., 2017). TGS is assumed to be more important than PTGS in the antiviral defence against geminiviruses, since viral DNA in recovered tissue is hypermethylated and is associated with inactive chromatin markers (Ceniceros-Ojeda et al., 2016). In addition, plants deficient in elements of the RdDM pathway become hypersusceptible (Jackel et al., 2016; Raja et al., 2008; Raja et al., 2014). Interestingly, RDR2 knock out plants are not hypersusceptible against geminiviruses and vsiRNA profiles against the geminivirus cabbage leaf curl virus (CaLCUV) are not remarkably changed in RDR1,2,6 triple knock out plants compared to wild type plants (Aregger et al., 2012; Raja et al., 2008). This suggests that RDR1, 2 and 6 are not involved in the antiviral RNAi response against geminiviruses, and other RDRs may be implicated.

The cloning and identification of the Ty-1 dominant resistance gene from Solanum chilense against TYLCV unveiled a new class of (non-NBS-LRR) resistance genes (Verlaan et al., 2013). The gene encodes an RDR from the gamma class, to which RDR3, 4 and 5 belong, and distinct from RDR1, 2 and 6 of the alpha class (Wassenegger and Krczal, 2006). Until that moment, no function was assigned to any of the RDRs from the gamma class. Following studies revealed that Ty-1 enhances the antiviral RNAi response, as inferred from elevated levels of vsiRNAs and increased methylation of cytosine-residues in the viral DNA genome in Ty-1 bearing tomato (Butterbach et al., 2014). To further elucidate the role of Ty-1 in the biogenesis of vsiRNAs and in the antiviral response to TYLCV, in this study small RNAs (sRNAs) were purified from susceptible and resistant Ty-1 bearing tomato plants. Sequence analysis and the genomic distribution of vsiRNAs revealed enhanced transitive silencing and an interesting increase in the production of 22-nt vsiRNAs. 


\section{Results}

\section{Sample preparation for vsiRNA profiling}

Tomato lines containing Ty-1 were previously shown to contain increased amounts of vsiRNAs during TYLCV infection compared to susceptible MM plants. However, nothing was known yet on the exact role of Ty-1 in the biogenesis of vsiRNAs, i.e. on the size distribution of vsiRNAs and their genomic distribution profile. To determine the effect of the resistance gene $T y-1$ on vsiRNA profiles in more detail, sRNAs were purified from infected and uninfected susceptible and $T y-1$ encoding tomato plants and next analysed via deep sequencing. First, five 3-week old Ty-1 bearing tomato seedlings and susceptible tomato Moneymaker (MM) seedlings were either infected with TYLCV by agroinoculation or, as negative control, infiltrated with untransformed agrobacteria. As expected, during the progression of disease MM plants infected with TYLCV showed clear yellowing and curling of the top leaves (Fig. 3.1A), while infected Ty-1 plants did not show any symptoms, just like uninfected control plants. At 29 days post infection (dpi) systemic leaves were harvested. From each group three plants were used to collect three independent biological replicates. To confirm a successful infection, DNA was extracted and virus titers determined via qPCR. The fold difference in titer was calculated using the DeltaDeltaCt method (Livak and Schmittgen, 2001) (Fig. 3.1B). While viral titers in infected MM plants were high, those in the Ty-1 introgression line were $\sim 20$ fold reduced. No virus was detected in the mock control plants, which all was in accordance with previous results (Chapter 2).

\section{Identification of vsiRNA populations in MM and Ty-1 plants}

After having verified viral titers in the mock treated and virus-infected MM and Ty-1 tomato introgression line, systemic leaves collected from each treatment were used for the purification of sRNAs and subsequent library preparation. Deep sequencing of the sRNAs from three biological replicates of each treatment resulted in libraries containing in total between 9.5 to 11.8 million clean reads, varying in length from 15-70 nts (Table 3.1). The libraries of mock infected samples consisted almost fully 
of reads mapping to the tomato genome (average $97,7 \%$ ) and with $0 \%$ of the reads mapping to the viral genome (Fig. 3.2A, Table 3.1). siRNAs mapping to the viral genome (vsiRNAs) in samples of TYLCV infected plants represented on average $4,0 \%$ and $3,1 \%$ of total reads in $\mathrm{MM}$ and Ty-1 plants, respectively (Fig. 3.2A). The amount of viral reads was comparable between the MM and Ty-1 plants, while previously an increased amount of vsiRNAs in Ty-1 plants compared to MM was reported (Butterbach et al., 2014). However, it is important to note the lower viral titer determined in Ty-1 plants. When the ratio of viral reads relative to the viral titer was determined, a strong increase in vsiRNAs per viral genome was observed in Ty-1 plants (Table 3.1, Fig. 3.2B).

\section{Increased vsiRNAs of 22 and 24-nt size classes in Ty-1 plants}

To further characterize the sRNAs, the size distribution was determined within the total pool of sRNAs. The most abundant sRNAs, and observed in samples from all treatments, were from the $24-n$ t size class, covering about $42 \%-54 \%$ of all sRNA reads (Fig. 3.3A, Sup. Fig. 3.1A). Although many studies often primarily focus on the function of sRNAs within the size range 21-24-nt, it was interesting to see that all libraries also revealed a relative smaller peak of 32-nt sRNAs (0,9-1,5\%) that were more abundant than those within the range between 26-31 nt. Furthermore, TYLCV infected Ty-1 plants also revealed a small peak of 44-nt sRNAs (0,5\%) (Sup. Fig 3.1A). When the sRNA reads were split into those mapping to the tomato genome versus the vsiRNA reads, the 32- and 44-nt sRNAs turned out to originate from the host (Sup. Fig. 3.1B).

The sRNA reads were next split into those sRNAs mapping to the tomato genome and those mapping to the TYLCV genome (vsiRNAs). Also among the tomato specific sRNAs, the $24-n t$ size class was by far the most prevalent, representing $54 \%$ of all sRNAs in uninfected MM, 53\% in uninfected Ty-1 plants, $48 \%$ in TYLCV-infected MM plants, and 46\% in TYLCV infected Ty-1 plants (Fig. 3.3B). Besides the 24-nt sRNAs, the other most frequently found sRNAs were from 23-nt, 22-nt and 21-nt size class, and which were all present in relative comparable amounts (8-16\%) (Fig. 3.3B). vsiRNAs were only collected from the TYLCV infected leaf samples and not 
A.

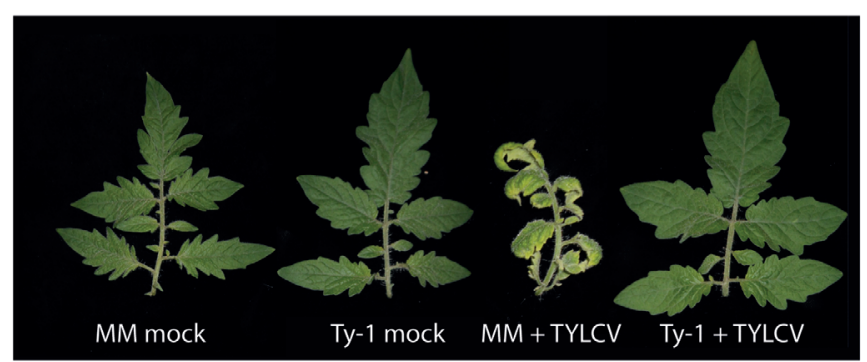

B.

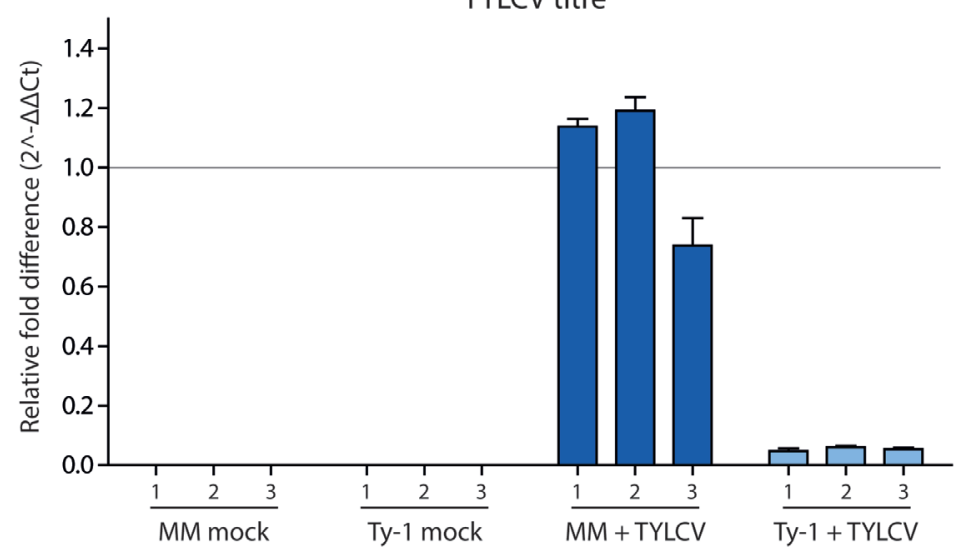

Figure 3.1: Symptoms and viral titers in $\mathrm{MM}$ and Ty-1 bearing tomato.

A. Symptoms upon infection with TYLCV or mock infection in respectively Ty-1 introgression tomato and susceptible MM at 29 days post infection. B. Quantification of TYLCV titer in MM and the Ty-1 introgression line. Values were normalized to actin and calibrated to the levels in MM plants (set to 1). Every bar represents the average viral titer and standard deviation based on technical replicates of individual plants.

found, as expected, in the mock infected plants. The most predominant class of vsiRNAs collected from susceptible MM, were from 21-nt (37\%), followed by 22 -nt (30\%) and the less abundant classes of 24-nt (8\%) and 23-nt (5\%) (Fig. 3.3B, Sup. Fig. 3.1B). In Ty-1 plants, this pattern was clearly changed, with the largest size class presented by 22 -nt (38\%), followed by 21 -nt (18\%), 24-nt (14\%), and 23-nt (9\%) (Fig. 3.3B, Sup. Fig. 3.1B). So, the class of the 21 -nt vsiRNAs was reduced by $\sim 50 \%$ in Ty-1 tomato compared to the susceptible MM, while the 22-nt, 23-nt and 24-nt size classes were clearly increased ( $25 \%$ increase for 22 -nt and $\sim 75 \%$ increase for 23- and 24-nt vsiRNAs). 


\begin{tabular}{|c|c|c|c|c|c|}
\hline Sample & $\begin{array}{l}\text { Total Clean } \\
\text { reads }\end{array}$ & $\begin{array}{l}\text { Reads mapped to } \\
\text { Tomato }\end{array}$ & $\begin{array}{l}\text { Reads mapped to } \\
\text { TYLCV }\end{array}$ & $\begin{array}{l}\text { Viral titer } \\
\text { (average } \\
\text { titer } \\
\text { MM+TYLCV } \\
\text { set to 1) }\end{array}$ & $\begin{array}{l}\text { TYLCV } \\
\text { reads } \\
\text { relative } \\
\text { to titer } \\
\text { (reads/ } \\
\text { viral titer) }\end{array}$ \\
\hline MM Mock 1 & 10.297 .567 & $10.082 .269(97,91 \%)$ & $141(0,00 \%)$ & 0,00 & \\
\hline MM Mock 2 & 11.218 .593 & $10.982 .695(97,90 \%)$ & $60(0,00 \%)$ & 0,00 & \\
\hline MM Mock 3 & 10.217 .804 & $10.024 .767(98,11 \%)$ & $92(0,00 \%)$ & 0,00 & \\
\hline Ty-1 Mock 1 & 11.840 .147 & $11.537 .941(97,45 \%)$ & $45(0,00 \%)$ & 0,00 & \\
\hline Ty-1 Mock 2 & 10.767 .517 & $10.486 .410(97,39 \%)$ & $220(0,00 \%)$ & 0,00 & \\
\hline Ty-1 Mock 3 & 10.992 .886 & $10.724 .901(97,56 \%)$ & $23(0,00 \%)$ & 0,00 & \\
\hline MM + TYLCV 1 & 10.211 .664 & $9.631 .616(94,32 \%)$ & $422.028(4,13 \%)$ & 1,14 & 370.956 \\
\hline MM + TYLCV 2 & 10.683 .014 & $10.049 .426(94,07 \%)$ & $441.441(4,13 \%)$ & 1,19 & 370.620 \\
\hline $\mathrm{MM}+\mathrm{TYLCV} 3$ & 10.048 .791 & $9.482 .625(94,37 \%)$ & 381.275 (3,79\%) & 0,74 & 516.655 \\
\hline Ty-1 + TYLCV 1 & 11.453 .220 & $10.907 .151(95,23 \%)$ & $283.914(2,48 \%)$ & 0,05 & 6.022 .338 \\
\hline Ty-1 + TYLCV 2 & 9.553 .480 & $9.048 .851(94,72 \%)$ & $279.112(2,92 \%)$ & 0,06 & 4.611 .120 \\
\hline Ty-1 + TYLCV 3 & 9.893 .962 & $9.283 .591(93,83 \%)$ & $384.052(3,88 \%)$ & 0,05 & 7.115 .461 \\
\hline
\end{tabular}

MM or Ty-1 bearing tomato plants were infected with TYLCV and 29 days post infection, sRNAs were isolated from systemic leaves and sequenced. Data from three biological replicates are shown. The amount of raw reads and the amount of reads mapped to either the tomato or TYLCV genome using Bowtie2 are depicted.

Having determined the size distribution of the vsiRNAs, the polarity of the reads was analysed. This was done for the total viral reads, and the 21-nt, 22-nt and 24-nt size classes. In MM plants, the majority of all viral reads (53\%) and those of the size class 21-nt (54\%) and 22-nt (54\%) were in sense polarity (clockwise orientation of the viral genome), while of the 24 -nt size class $46 \%$ was in sense polarity (Fig. 3.4). In Ty-1 tomato, the amount of sense reads showed a small reduction of 3-6\% in all the studied size classes when compared to MM plants, with $47 \%$ of all viral reads, $48 \%$ of 21 -nt reads $51 \%$ of 22 -nt reads and $40 \%$ of $24-n t$ reads being in sense polarity (Fig. 3.4).

In light of the importance of the $5^{\prime}$ terminal nucleotide of siRNAs toward loading into different AGO proteins (Kim, 2008; Mi et al., 2008), the 5' terminal nucleotide 
A.

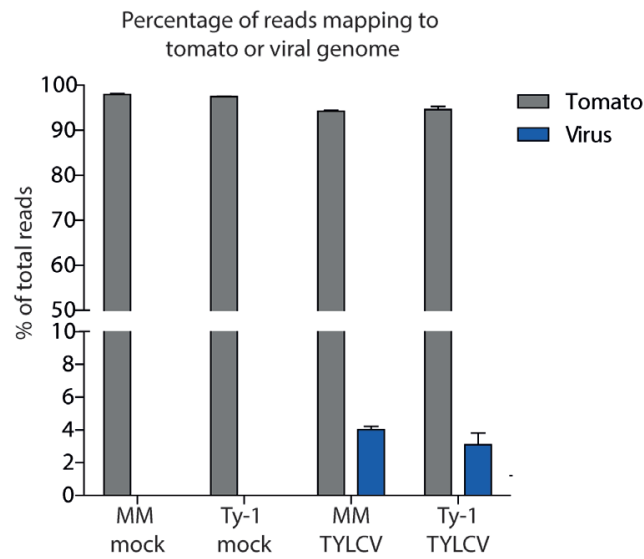

B.

vsiRNA reads relative to viral titer

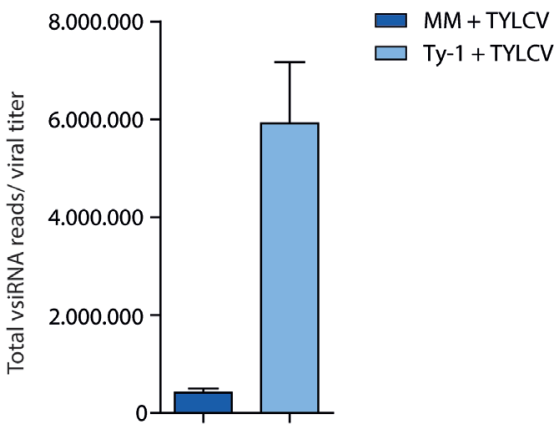

Figure 3.2: Distribution of sRNAs mapping to the tomato genome or viral genome.

A. sRNAs were isolated from mock-treated and TYLCV-infected susceptible MM and Ty-1 bearing tomato and subsequently sequenced. The sRNAs were mapped to either the tomato genome or the viral genome using Bowtie2. The percentage of reads mapping to either the tomato or TYLCV genome relative to the total reads is depicted. Each bar represents the mean percentage and standard deviation of three biological replicates. B. The number of sRNAs mapped to the viral genome was corrected for the relative amount of virus found in the samples according to qPCR. The bars represent the mean and standard deviation of three biological replicates.

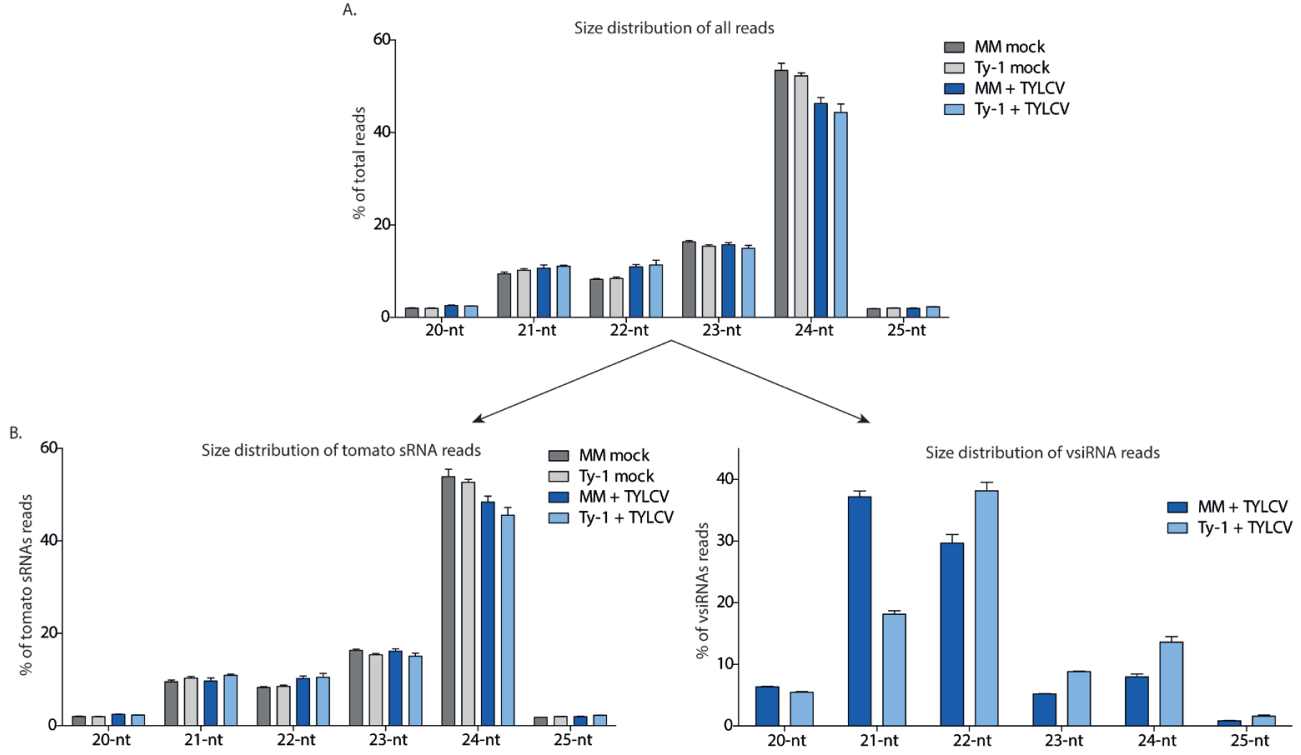

Figure 3.3: size distribution of the total sRNAs, sRNAs mapping to the tomato genome and siRNAs mapping to the viral genome (vsiRNAs).

The sRNAs reads of the four groups (MM and Ty-1 tomato, mocked treated and TYLCV-infected) were analysed on size. A. The percentage for each size class (20-25 nt) on the total amount of reads is plotted. 
B. The library of all reads was split into host-derived sRNAs and vsiRNAs and the percentage for each size class (20-25 nt) is depicted relative to the total amount of reads mapping to the tomato genome (left panel), and relative to the total amount of reads mapping to the viral genome (right panel). Viral reads are only analysed from TYLCV infected plants (right panel).

of viral reads was analysed. In both MM and Ty-1 tomato reads from the 21-nt and 22-nt classes were enriched in a $5^{\prime}$ terminal uridine (U), with a prevalence around $40 \%$ in both groups. The second most dominant 5 'terminal nucleotide was adenosine (A), with an average prevalence around $27 \%$ in both groups (Fig. 3.5). Within the 24-nt reads, A was observed most prevalent ( $40 \%)$, followed by $U$ ( 30\%) (Fig. 3.5). The same patterns were observed when reads of viral sense and antisense polarity were separately analysed (Sup. Fig. 3.2), with the exception of two cases. Within the 21-nt and 22-nt reads of antisense polarity $U$ and A were more equally presented at the $5^{\prime}$ end, while $U$ was clearly dominant within the sense reads. The 24-nt reads of sense polarity showed a comparable proportion of $U$ and $A$ as 5 'terminal nucleotide, while $A$ was more dominant in the reads of antisense polarity (Sup. Fig. 3.2). No clear differences were observed in $5^{\prime}$ terminal nucleotides between vsiRNAs isolated from MM and Ty-1 plants.

\section{TYLCV is targeted by vsiRNAs covering the entire viral genome in MM and Ty-1 plants}

To examine the genomic distribution of vsiRNAs produced and analyse for possible changes in the presence of Ty-1, the reads derived from infected susceptible MM and resistant Ty-1 tomato were plotted against the TYLCV genome. The results showed that in both MM and Ty-1 plants the vsiRNAs cover the entire genome in viral sense and antisense orientation (Fig. 3.6). For both MM and Ty-1 plants, the genomic distribution was somewhat heterogenous, with several regions being covered by more and others by less vsiRNAs. This was reflected in all vsiRNA size classes (Fig. 3.6) and observed highly consistent in all three biological replicates (Sup. Fig 3.3). Hotspots, regions with a higher density of vsiRNAs per nucleotide, were visible in the viral genome distribution of both MM and Ty-1 tomato, but these hotspots appeared more distinct in MM. Hotspots coincided with regions of overlapping open reading frames (ORFs), i.e. $\mathrm{V} 1 / \mathrm{V} 2, \mathrm{C} 1 / \mathrm{C} 2 / \mathrm{C} 3$, and $\mathrm{C} 1 / \mathrm{C} 4$ 


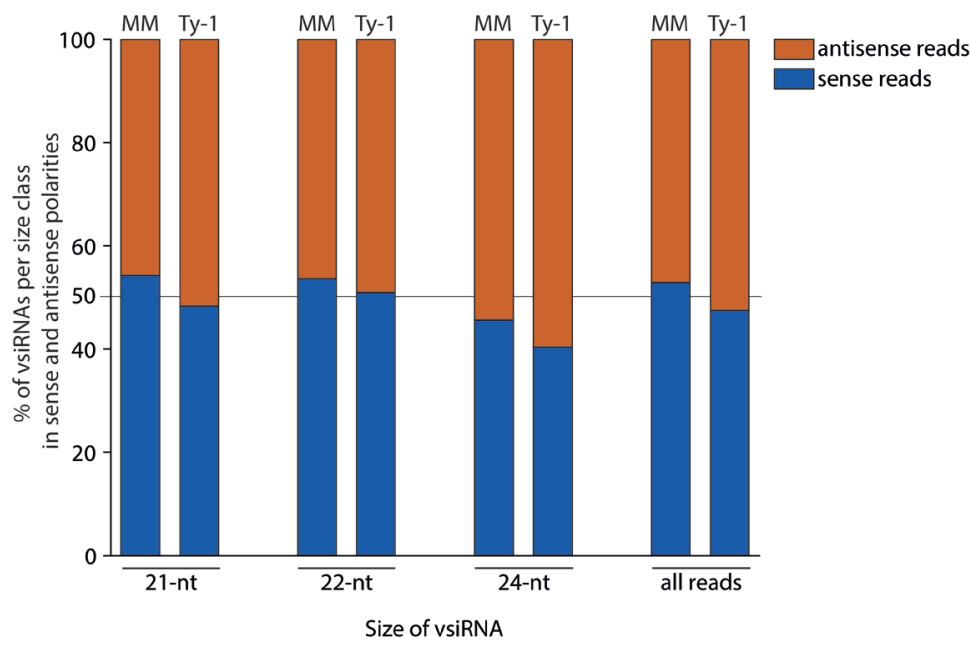

Figure 3.4: The polarity of vsiRNA reads (of different size classes).

Viral siRNAs from TYLCV-infected MM or Ty-1 bearing tomato depicted per size class (21-nt, 22-nt, 24-nt or all reads) and showing the percentage of sense and antisense oriented reads. The average of three biological replicates is depicted.

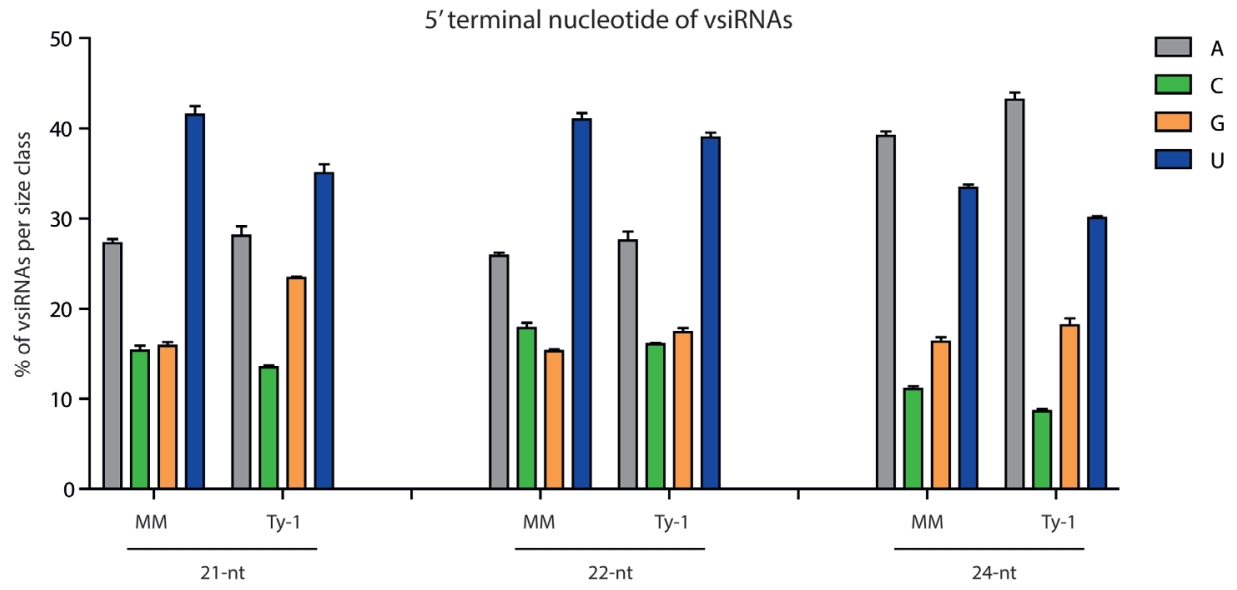

Figure 3.5: Distribution of the 5' terminal nucleotide of vsiRNA reads from MM and Ty-1 tomato.

Viral siRNAs from TYLCV-infected MM or Ty-1 tomato depicted per size class of 21-nt, 22-nt and 24-nt and showing the percentage of reads containing adenine $(A)$, cytosine $(C)$, guanine $(G)$, or uracil $(U)$ as $5^{\prime}$ terminal nucleotide. The bar represents the average and standard deviation of three biological replicates. 
respectively (Fig. 3.6, Table 3.2A), and were present in both the viral sense and antisense orientation and observed for all vsiRNA size classes. Only in the profile of 24-nt vsiRNAs, the hotspot at the $\mathrm{C} 1 / \mathrm{C} 2 / \mathrm{C} 3$ overlapping region was less pronounced.

In both $\mathrm{MM}$ and Ty-1 tomato the intergenic region (IR) had the lowest abundance of vsiRNAs (Table 3.2A). Other regions with a low abundance in MM plants included the V2 start (5'end of the V2 ORF until the start of V1 ORF), the V1-C3 junction (where the 3'ends of the $\mathrm{V} 1$ and $\mathrm{C} 3$ transcripts are assumed to overlap), and the middle part of $\mathrm{C} 1$ (between the end of $\mathrm{C} 4$ and the start of $\mathrm{C} 2$ ) (Fig. 3.6, Table 3.2A).

Not only the distribution of vsiRNA in all size classes was changed in the presence of Ty- 1 , the amount of reads per size class in MM and Ty-1 plants was also changed (Fig. 3.3B). This complicated the comparison of the genome distribution between the two groups. Therefore, per size class, the percentage of mapped vsiRNAs per nucleotide was plotted (Sup. Fig. 3.4). The three hotspots earlier observed at the regions with overlapping ORFs, were again visible. However, when the proportion of vsiRNAs within the size class 21,22 , and 24-nts mapping to these hotspots was calculated, a higher amount was obtained for MM (55\%) compared to Ty-1 tomato (41\%), indicating that in Ty-1 tomato the hotspots were less targeted. To further quantify the observed differences in vsiRNA genome distribution between MM and Ty-1 tomato, the number of reads/nucleotide was calculated for several genomic regions and compared to the reads/nucleotide in the situation of a completely homogenous distribution (Table 3.2A). The results strengthened the observation of less pronounced hotspots at the ORF overlapping regions of TYLCV in Ty-1 tomato compared to the situation in MM. Interestingly, the region at the 3'end of the V1 and C3 ORF (V1-C3 junction), which has a low abundance of vsiRNA in MM, shows to have a higher abundance than average in Ty-1 plants (see Table 3.2A, region "V1-C3 junction"). The increased density at V1 was in agreement with an earlier study, where the ratio of vsiRNAs mapping to $\mathrm{V} 1 / \mathrm{C} 3$ changed in the presence of Ty-1 (Butterbach et al., 2014). When the ratio was calculated for the same regions as used in the previous study, a comparable shift was observed (Table 3.2B).

To better visualise the change in genome distribution and obtain a broader overview image, the amount of reads per 100 nucleotides were plotted (Fig. 3.7). Also the 
MM

21-nt vsiRNAs $(37,2 \%)$
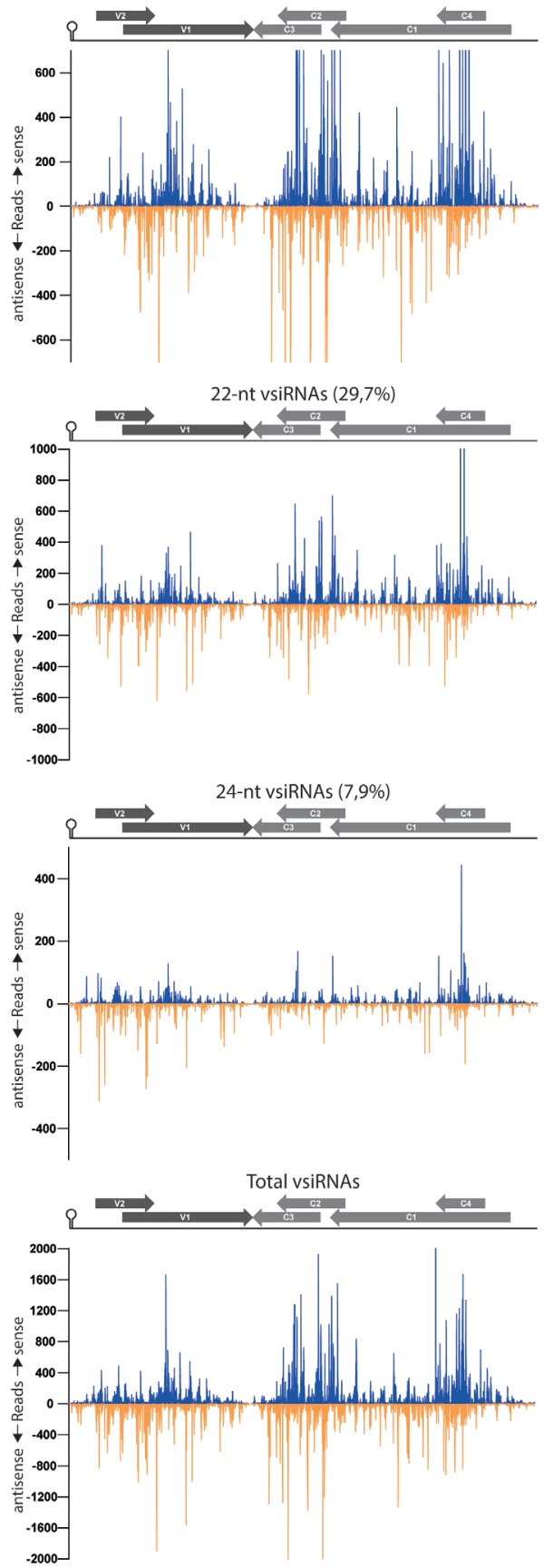

Ty-1

21-nt vsiRNAs (18,1\%)
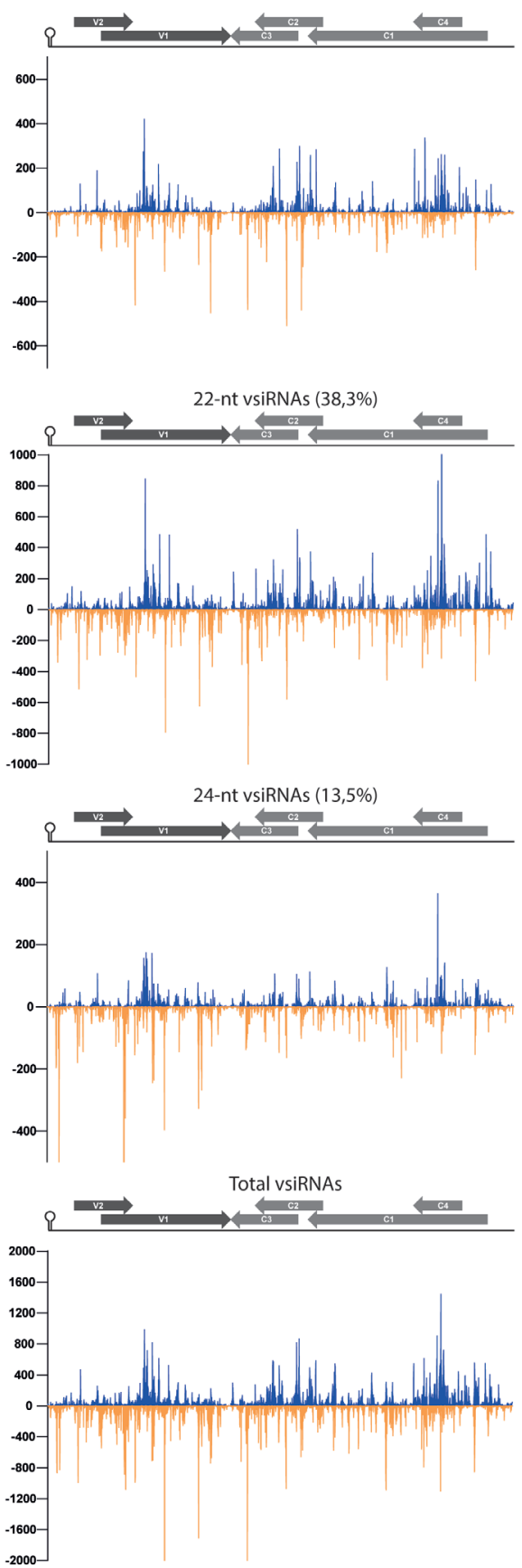
Figure 3.6: Distribution of vsiRNAs on the TYLCV genome in MM and Ty-1 tomato

Viral siRNAs isolated from infected MM (left panel) or Ty-1 (right panel) tomato were mapped on the genome of tomato yellow leaf curl virus (TYLCV). The number of reads at each nucleotide position of the TYLCV genome is plotted (average of three biological replicates), for either 21-nt, 22-nt, 24-nt, and for all vsiRNAs. Blue bars represent sense reads starting at each respective nucleotide, while the orange bars represent antisense reads ending at that position. The depicted percentage in the title represents the proportion that belongs to the size class relative to all vsiRNAs in either MM or Ty-1 tomato. A schematic overview of the TYLCV genome is depicted above the graphs, with the viral ORFs indicated as grey arrows and the intergenic region at the left and right side.

percentage of mapped vsiRNAs per 100 nucleotides was plotted to compare the two groups without a possible bias by a difference in absolute reads (Sup. Fig. 3.5). Those plots confirmed the previous finding of high density of vsiRNAs at the regions with the overlapping ORFs V1/V2 and C1/C4 in both MM and Ty-1 plants. It also revealed that in Ty-1 plants less vsiRNAs accumulated at the region with the overlapping ORFs $\mathrm{C} 1 / \mathrm{C} 2 / \mathrm{C} 3$, while this region is a clear hotspot in $\mathrm{MM}$ (Sup. Fig. 3.5, Table 3.2A). In addition, the amount of vsiRNAs that were mapped to the IR and to V1 was clearly increased in Ty-1 plants, especially in antisense orientation (Sup. Fig. 3.5, Table 3.2A).

\section{Targeting of Intergenic Region of TYLCV}

Comparison of the genomic distribution profiles of the vsiRNAs collected from susceptible $\mathrm{MM}$ and resistant Ty-1 tomato indicated that the IR and the region "V1-C3 junction" were relatively more targeted in Ty-1 tomato. The percentage of vsiRNAs to those regions were increased 3 and 1,5 fold, respectively (Table 3.2A). A previous study reported that in MM plants the IR was targeted relatively more by vsiRNAs of 24-nt than by those of 21-nt and 22-nt (Piedra-Aguilera et al., 2019). A closer look at the data from this study confirmed these observations (Table 3.2C). This study in addition showed that in Ty-1 tomato, the amount of vsiRNAs mapping to the IR increased for all three size classes (21-nt, 22-nt and 24-nt) in both polarities, but with highest amounts of antisense vsiRNAs (Table 3.2C). In both MM and Ty-1 tomato, the antisense reads of 24 -nts vsiRNAs were most prevalent (3.6\% and $6.1 \%$, respectively, Table $3.2 \mathrm{C}$ ). 
Table 3.2: Distribution of vsiRNAs on the TYLCV genome.

A.

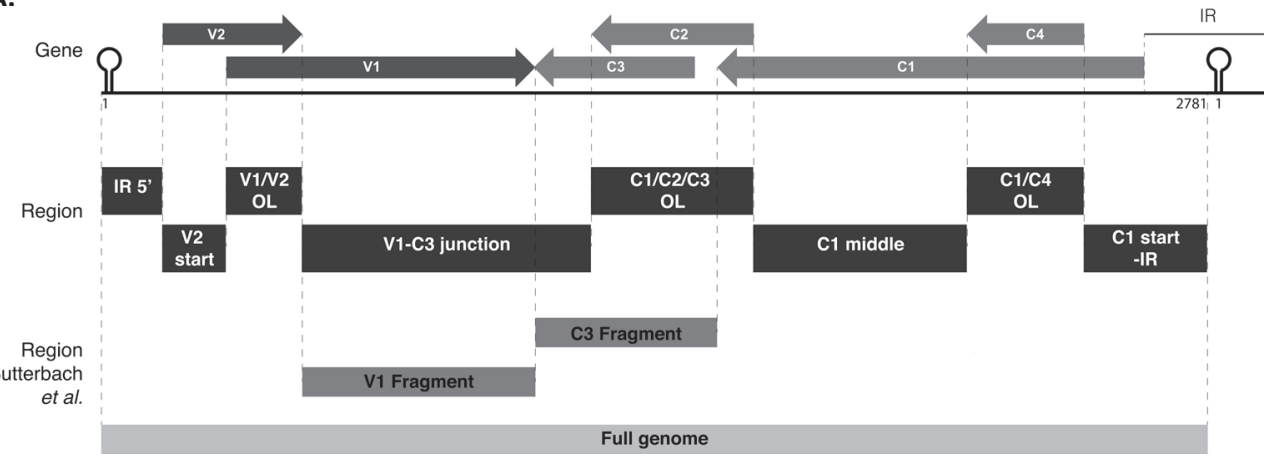

\begin{tabular}{|c|c|c|c|c|c|c|c|}
\hline \multirow[t]{2}{*}{ Gene } & \multicolumn{2}{|c|}{ vsiRNAs (reads) } & \multicolumn{2}{|c|}{$\begin{array}{c}\text { vsiRNA reads/ } \\
\text { nct }\end{array}$} & \multicolumn{2}{|c|}{$\begin{array}{l}\text { siRNA reads per nct / } \\
\text { average vsiRNAs per nct } \\
(>1 \text { is more siRNAs per nt } \\
\text { than on average over full } \\
\text { genome) }\end{array}$} & \multirow{2}{*}{$\begin{array}{l}\text { Change } \\
\text { in Ty-1 } \\
\text { compared } \\
\text { to MM } \\
\text { Ty-1/MM }\end{array}$} \\
\hline & MM & Ty-1 & MM & Ty-1 & MM & Ty-1 & \\
\hline V1 & 78469 & 69531 & 101,0 & 89,5 & 0,90 & 1,13 & 1,25 \\
\hline V2 & 38170 & 23722 & 108,7 & 67,6 & 0,97 & 0,85 & 0,88 \\
\hline C1 & 131224 & 85935 & 122,2 & 80,0 & 1,09 & 1,01 & 0,92 \\
\hline $\mathrm{C} 2$ & 91725 & 41039 & 224,8 & 100,6 & 2,01 & 1,27 & 0,63 \\
\hline C3 & 64122 & 36601 & 158,3 & 90,4 & 1,42 & 1,14 & 0,80 \\
\hline C4 & 56298 & 33759 & 191,5 & 114,8 & 1,72 & 1,45 & 0,84 \\
\hline IR & 6398 & 13679 & 20,4 & 43,7 & 0,18 & 0,55 & 3,01 \\
\hline \multicolumn{8}{|l|}{ Region } \\
\hline IR 5' & 3702 & 6780 & 24,0 & 44,0 & 0,22 & 0,56 & 2,58 \\
\hline V2 start & 14090 & 8051 & 88,1 & 50,3 & 0,79 & 0,63 & 0,80 \\
\hline V1/V2 OL & 24080 & 15671 & 126,1 & 82,0 & 1,13 & 1,04 & 0,92 \\
\hline V1-C3 junction & 61472 & 63886 & 84,6 & 87,9 & 0,76 & 1,11 & 1,46 \\
\hline $\mathrm{C} 1 / \mathrm{C} 2 / \mathrm{C} 3 \mathrm{OL}$ & 91725 & 41039 & 224,8 & 100,6 & 2,01 & 1,27 & 0,63 \\
\hline C1 middle & 48577 & 32755 & 90,5 & 61,0 & 0,81 & 0,77 & 0,95 \\
\hline C1/C4 OL & 56298 & 33759 & 191,5 & 114,8 & 1,72 & 1,45 & 0,84 \\
\hline C1 start-IR & 10494 & 18510 & 33,9 & 59,7 & 0,30 & 0,75 & 2,48 \\
\hline Full genome & 310437 & 220450 & 111,6 & 79,3 & 1,00 & 1,00 & \\
\hline
\end{tabular}


B. Regions corresponding to Butterbach et al.

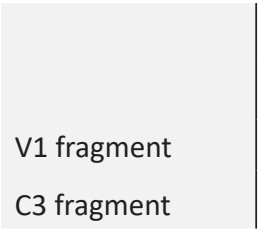

\begin{tabular}{r|} 
MM \\
54389 \\
80255
\end{tabular} )
Ty-1
43256

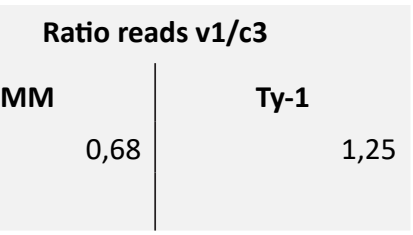

C. Percentage Intergenic Region (IR) specific vsiRNAs of viral siRNAs of that size class.

\begin{tabular}{l|c|c|c|c|c|c} 
& \multicolumn{2}{|c|}{ 21nt } & \multicolumn{2}{c|}{ 22nt } & \multicolumn{2}{c}{ 24nt } \\
& $(+)$ & $(-)$ & $(+)$ & $(-)$ & $(+)$ & (-) \\
\hline MM & 0,53 & 0,66 & 0,93 & 1,32 & 1,78 & 3,64 \\
Ty-1 & 1,64 & 2,43 & 2,83 & 3,62 & 2,43 & 6,06
\end{tabular}

A. MM or Ty-1 bearing tomato were infected with TYLCV and 29 days post infection, sRNAs were isolated from systemic leaves and sequenced. The average of three biological replicates is shown. On top the viral genome is schematically depicted with different genes and genomic regions indicated by arrows and blocks, respectively. In the table the number of vsiRNA reads of 21-nt, 22-nt and 24-nt mapping to these genomic positions (as depicted in figure 3.6) are shown. The density (vsiRNA reads/nucleotide) is calculated by dividing the amount of reads by the length of the genomic region or gene (column 4 for MM and column 5 for Ty-1). Column 6 (MM) and 7 (Ty-1) depict for the same regions whether they have a higher or lower density than on average over the full genome. This is calculated by dividing the vsiRNA reads/nucleotide with the average amount of reads/nucleotide. In bold the regions with a higher density are indicated. In the last column the change in Ty-1 compared to MM is depicted, with regions in bold that contain a higher density in Ty-1 compared to MM. B. The number of reads (21-nt, 22-nt and 24-nt) mapping to two fragments earlier studied on the amount of vsiRNAs by Butterbach et al. (Butterbach et al., 2014) are indicated, and the ratio $V 1 / C 3$ is calculated based on the amount of reads from this study. C. The percentage of 21-nt, 22-nt and 24-nt, respectively, mapping to the intergenic region (nucleotide 2623-154) of TYLCV in MM and Ty-1 bearing tomato. The depicted percentage represents the vsiRNA reads of each size class mapping to the Intergenic region in either viral sense (+) of antisense (-) orientation, relative to the total vsiRNA reads of that size class. The average of three biological replicates is shown.

To analyse whether the increased amount of vsiRNAs from the IR and "V1-C3 junction" region mapped to specific sequences (e.g. secondary structures) or were distributed comparable to the situation in $\mathrm{MM}$, genomic distribution graphs were made zoomed in on these regions (Fig. 3.8, Sup. Fig. 3.6). In the region "V1-C3 junction" the increased amounts of vsiRNAs from Ty-1 tomato distributed all over the region (Fig. 3.8B, Sup. Fig. 3.6B). In contrast, vsiRNAs from the IR appeared to concentrate in two "sub-regions", namely around nucleotides 2624-2665 and nucleotides 41-84 of the TYLCV genome (Fig. 3.8A, Sup. Fig. 3.6A). Earlier, reports of 
resistance breaking strains in Ty-1 tomato described recombination events between TYLCV and Tomato yellow leaf curl Sardinia virus (TYLCSV) taking place in the IR region (Belabess et al., 2015; Belabess et al., 2016; Belabess et al., 2018; Torre et al., 2018; Urbino et al., 2020), and with a smallest recombination event reported for the resistance breaking TYLCV-IS76 (Urbino et al., 2020). To analyse whether this region coincided with the hotspot "sub-regions" of the IR found in this study, the region of recombination in TYLCV-IS76 was compared with the sub-regions of increased vsiRNAs in Ty-1 plants. The results showed that one region (nucleotide 41-84) indeed overlapped with the recombination event (Fig. 3.8A).

\section{Potential production of dicer independent siRNAs}

Recently, an alternative Dicer-independent pathway resulting in genome methylation was discovered (Ye et al., 2016). In this process, longer RNA molecules bind to AGO4 and are subsequently trimmed, independent of Dicer, by $3^{\prime}$ to $5^{\prime}$ exonucleases into siRNAs. The population of siRNAs resulting from this show up as a "ladder" pattern ( 20-60 nt in size) and share the same 5' end but differ at their 3' end with one nucleotide. Since not much is known yet on the role of members from the RDRy in (viral) siRNA biogenesis, and whether these would assist in the production of dicer independent siRNAs (sidRNA), the reads collected from MM and Ty-1 tomato were examined for molecules larger than 24-nt and matching features of sidRNAs. In both MM and Ty- 1 tomato TYLCV specific vsiRNAs $>24$ nt were found, but the amounts of these were not substantially increased in the presence of Ty-1 (Sup. Fig. 3.7A). Spots with potential sidRNAs were found by screening for vsiRNAs 25-33 nt in size and sharing the same 5'end (for an example of a potential sidRNA see Sup. Fig. 3.7C). The potential sidRNA spots were mostly in antisense orientation and some were even consistently found in biological replicates, although the positions identified in Ty-1 plants were different from those in MM plants (Sup. Table 3.1, Sup. Fig. 3.7D-F). However, in general their number was relatively low, i.e. 11 and 3 from MM and Ty-1 plants, respectively (Sup. Fig. 3.7B). 

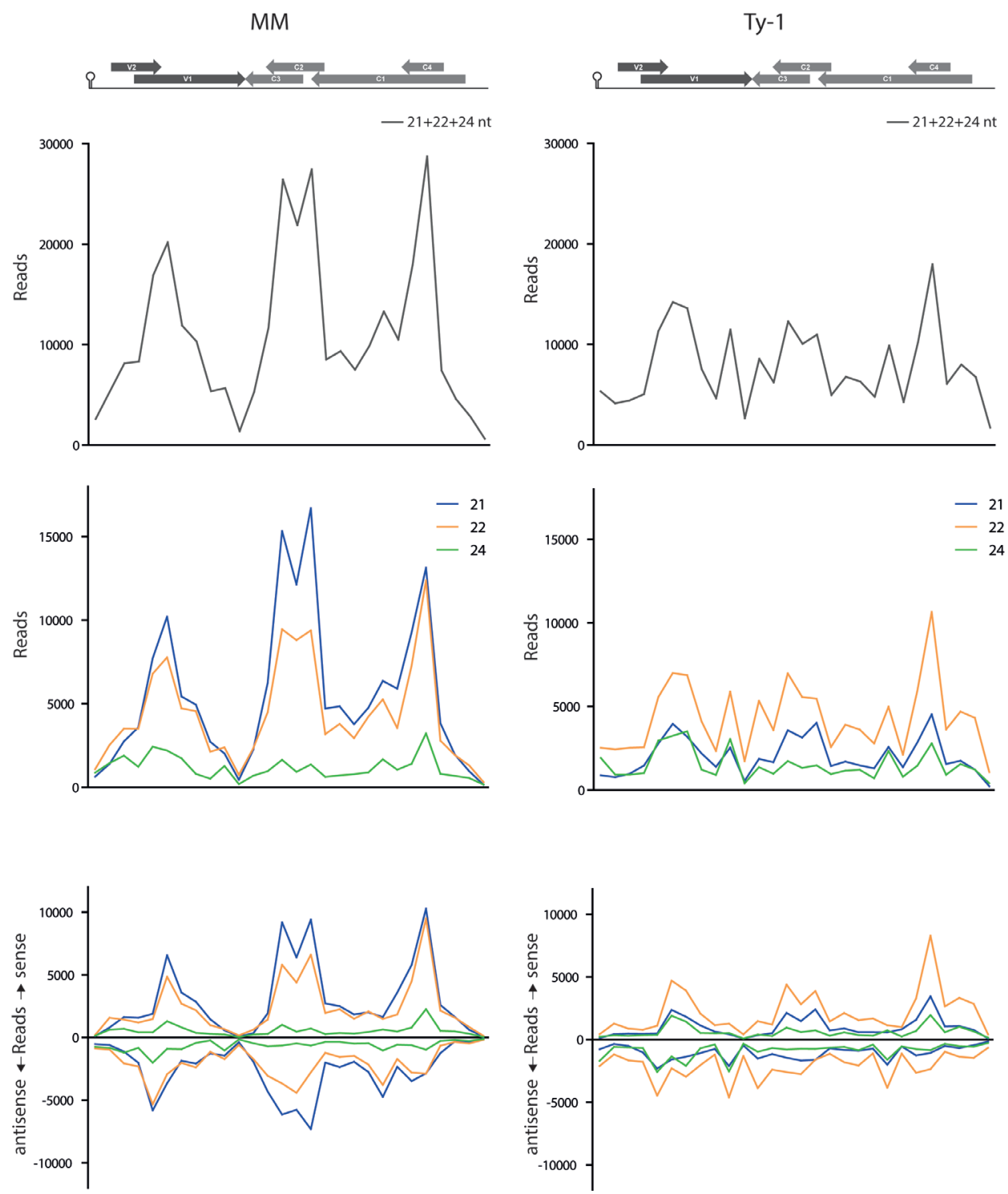

Figure 3.7: Amount of vsiRNA reads per 100 nucleotides in the TYLCV genome.

Viral siRNAs isolated from infected MM (left panel) or Ty-1 bearing (right panel) tomato are mapped on the genome of tomato yellow leaf curl virus (TYLCV). The numbers of reads per 100 nucleotides of the TYLCV genome (amount of reads for position 1-100, 101-200 etc.) are plotted. The graphs represent the average of three biological replicates. The upper panels present the amount of reads of 21-nt, 22-nt, and 24-nt size class together. The middle and lower panels depict the distribution of 21-nt, 22-nt and 24-nt vsiRNA separately, either sense and antisense polarities taken together (middle panel), or both polarities specified (lower panel). A schematic overview of the TYLCV genome is presented at the top of the graph, with the viral ORFs indicated as grey arrows and the intergenic region at the left and right side. 
A.

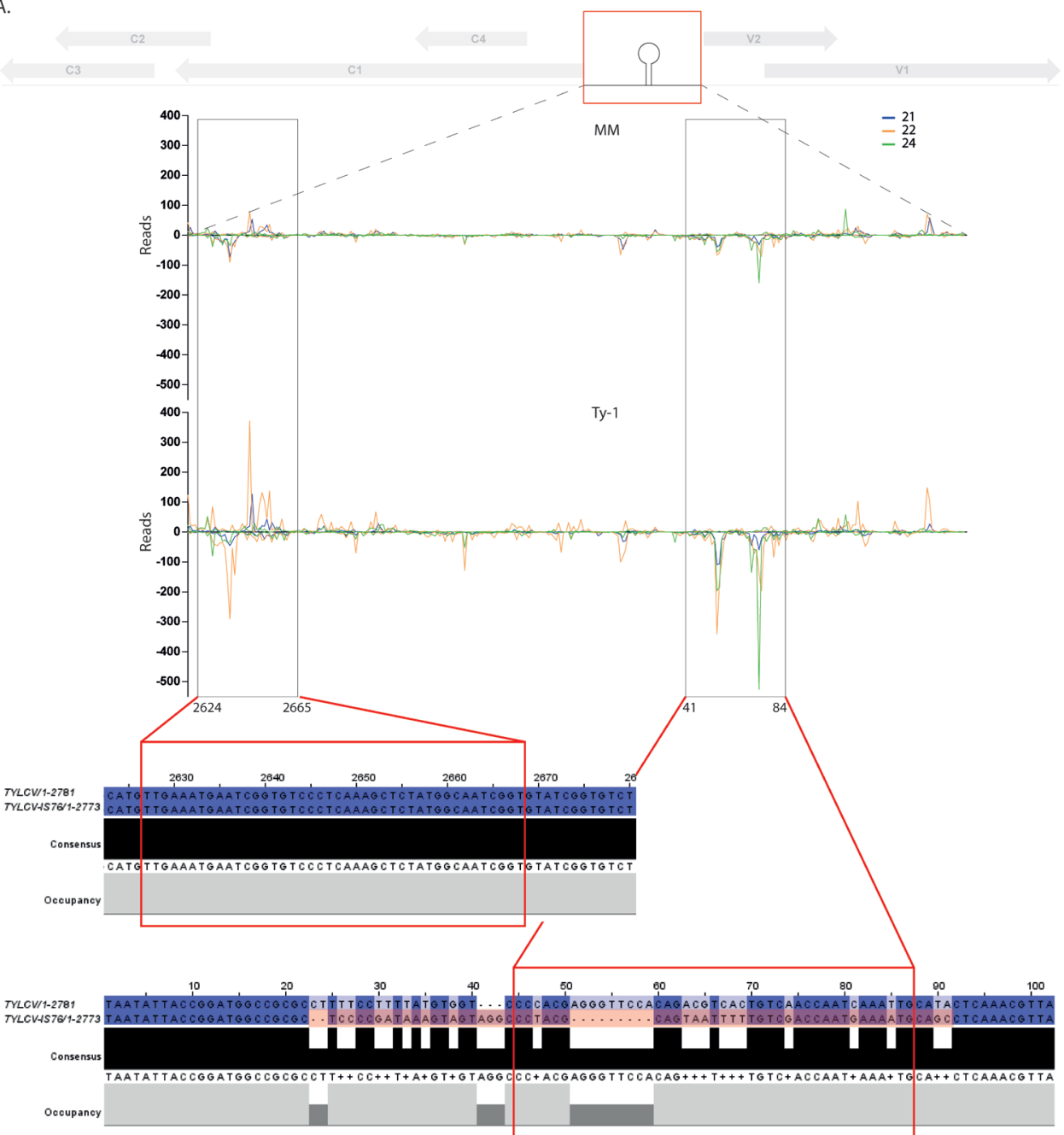

Figure 3.8: Close-up on the vsiRNA distribution in the intergenic region and "V1-C3 junction" and topological alignment of the recombinant IR region from TYLCV-IS76 with the vsiRNA of the intergenic region Viral siRNAs isolated from infected MM (top panel) or Ty-1 bearing (lower panel) tomato are mapped on the genome of tomato yellow leaf curl virus (TYLCV). The numbers of vsiRNA reads at each nucleotide position of the TYLCV genome are plotted (average of three biological replicates), for either 21-nt, 22-nt, and 24-nt vsiRNAs. Positive bars represent sense reads starting at each respective nucleotide, while the negative bars represent antisense reads ending at that position. A schematic overview of TYLCV genome is depicted at the top of each graph, with the viral ORFs indicated as grey arrows and the region zoomed in boxed in red. A. Genomic distribution of vsiRNAs in the intergenic region (nucleotides 2623-154). Regions of major differences between vsiRNA profiles of MM and Ty-1 plants are highlighted in black boxes and underneath the nucleotide sequences of the corresponding regions from TYLCV and the Ty-1 resistance-breaking TYLCV-IS76 are shown. B. (on next page) Genomic distribution of vsiRNAs in the region "V1-C3 junction" (nucleotides 506-1232) 
B.

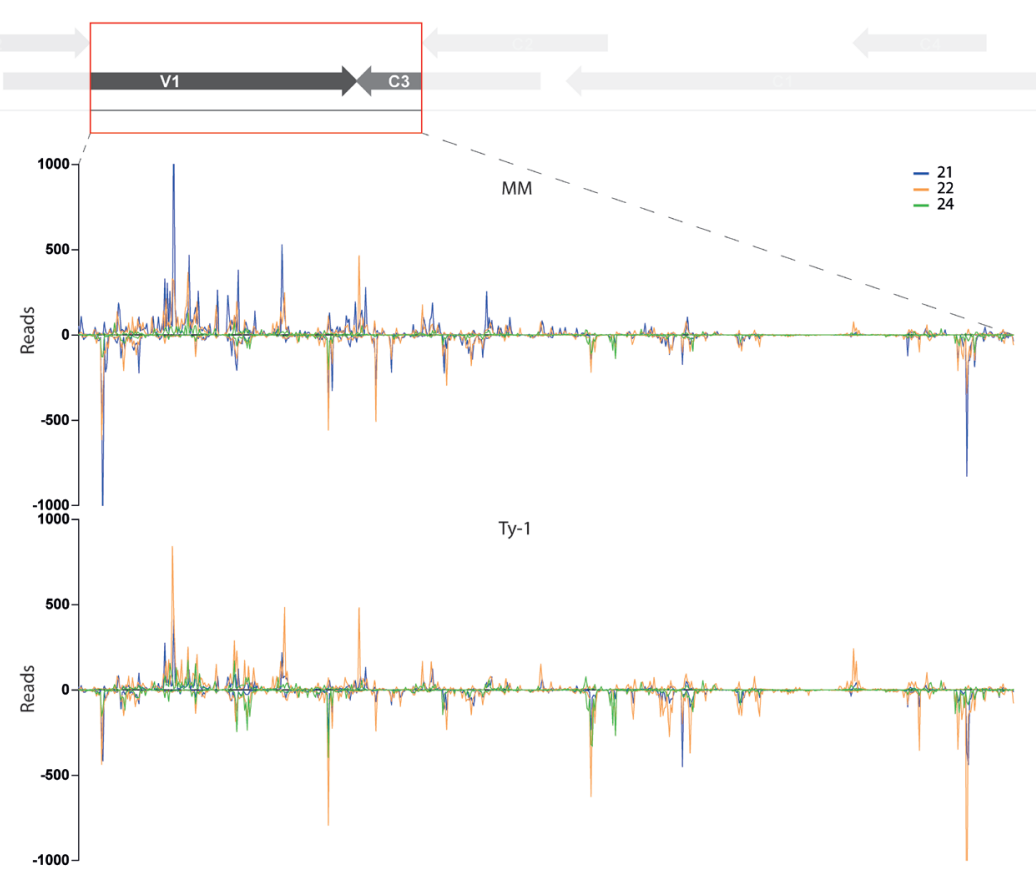

\section{Discussion}

The role of RDR1, 2 and 6, all three members of the RDR $\alpha$ class, in the biogenesis of siRNAs already has been well established (Willmann et al., 2011). In this study a first step was made to unravel the role of Ty-1, a dominant resistance gene protein against geminiviruses and member of the RDR $y$ class, in the biogenesis of siRNAs. To this end, susceptible MM and Ty-1 containing tomato were either mock infected or infected with TYLCV and comparatively analysed on the biogenesis of vsiRNAs. Comparable amounts of vsiRNAs were observed in MM and Ty-1 bearing plants, but the ratio of vsiRNAs to viral DNA copies in Ty-1 tomato was increased about $\sim 14$-fold. In susceptible MM vsiRNAs of 21-nt were found to be dominant, while in Ty-1 tomato vsiRNAs of 22-nt and 24-nt were increased and those of 21 nt were drastically reduced. Several vsiRNA hotspots were found, most of them mapping to genomic regions containing overlapping ORFs, but these hotspots were less pronounced in the presence of Ty-1. In Ty-1 tomato, additionally more vsiRNAs were found to target the IR and the V1 ORF. Altogether, these results support earlier findings in 
which Ty-1 seems to strengthen an antiviral TGS response. Whether the increased production of 22-nt vsiRNAs also plays a role in this, involving a (non-canonical) RdDM pathway, distinct from the RDR2-mediated RdDM pathway, or whether these contribute to an antiviral PTGS response still remains to be investigated.

The $5^{\prime}$ terminal nucleotide analysis revealed no difference between MM and Ty-1 plants. The dominant $5^{\prime}$ terminal nucleotide of vsiRNAs of 21-nt and 22-nt size class was $U$, followed by $A$. For the 24 -nt size class the predominant 5 ' terminal nucleotide was $\mathrm{A}$, followed by U. Argonaute proteins have been shown to preferably associate with siRNAs of certain size and 5' terminal nucleotide (Mallory and Vaucheret, 2010). The findings in this study are in agreement with 5' terminal nucleotide analyses of vsiRNAs as previously reported for TYLCV and other geminiviruses (Aregger et al., 2012; Piedra-Aguilera et al., 2019; Yang, Wang, et al., 2011), and supported the notion that geminiviral siRNAs are loaded into several AGO proteins. Furthermore, the prevalent $5^{\prime} U$ residue within the 22-nt vsiRNAs from Ty-1 tomato, similar to the $5^{\prime}$ end residue prevalence of 22-nt vsiRNAs from susceptible MM, does not make a clear distinction on the involvement of a (new Ty-1 mediated) non-canonical RdDM pathway with AGO4/AGO6 or the PTGS pathway. While the specificity of AGO4 and 6 is normally for 24-nt containing a 5'A and PTGS related siRNAs bound to AGO1 or AGO2 show a 5'U and 5'A bias respectively (Havecker et al., 2010; Mi et al., 2008), the AGO6 bound 22-nt siRNAs of the non-canonical pathway have less 5 'terminal nucleotide bias and contain both 5'A or 5'U (McCue et al., 2015). Therefore, further research is needed to explain the function of 22-nt vsiRNAs in TYLCV infected Ty-1 tomato.

While in MM three hotspots were clearly visible in the TYLCV genome at regions containing overlapping ORFs, they were less pronounced in the situation of Ty-1 tomato, with the number of vsiRNA counts per nucleotide in these regions less deviating from the average amount of vsiRNAs per nucleotide over the full genome (Table 3.2). This was also seen for regions with a lower number of vsiRNAs (coldspots), indicating that the vsiRNAs are more equally spread over the genome in Ty-1 tomato compared to MM. This is most likely explained by transitivity resulting from Ty-1. This process has earlier been described and starts with the conversion of (aberrant) single stranded RNAs, resulting from primary RNA target cleavage, into 
dsRNAs by the action of RDRs. Their subsequent processing by DCLs leads to a pool of secondary siRNAs and spreading of the siRNAs into the neighbouring sequences from the initial target sequences, called transitivity (de Felippes and Waterhouse, 2020). An alternative explanation is that in Ty-1 plants the expression pattern of viral genes might be changed, resulting in different amounts of templates to generate vsiRNAs.

An intriguing question that still remains to be solved is in which pathway Ty-1 functions. Since geminiviruses seem to be mostly affected by TGS and the methylation of the TYLCV genome is enhanced in Ty-1 tomato (Butterbach et al., 2014; Ceniceros-Ojeda et al., 2016; Jackel et al., 2016; Raja et al., 2008; Raja et al., 2014), it was hypothesized that Ty-1 amplifies the TGS response. Hence, an increase in the production of 24-nt vsiRNAs was expected. Interestingly, besides 24-nt also 22-nt vsiRNA levels were relatively increased, while those of 21-nt had clearly dropped (Fig. 3.3). Since 22-nt vsiRNAs are generally thought to function in the PTGS response and 24-nt vsiRNAs in the TGS response (Pumplin and Voinnet, 2013), the findings from this study would imply an increase of both pathways. However, alternative pathways leading to RdDM have been reported and are thought to be involved in the initiation of methylation. (Erdmann and Picard, 2020; Kenchanmane Raju et al., 2019). Some of these non-canonical RdDM pathways involve 21-22 nt siRNAs and several elements of the PTGS pathway. In one pathway, RDR6-produced dsRNA is processed into 21-22 nt siRNAs by DCL2/DCL4. Instead of being loaded into AGO1, the siRNA is loaded into AGO6 and directs the RdDM (McCue et al., 2015). Alternatively, dsRNA produced by RDR6 becomes processed by DCL3 in 24-nt siRNAs, and guide RdDM by AGO4 or AGO6 (Marí-Ordóñez et al., 2013). More recently, a Dicer-independent pathway has been described in which longer RNA molecules are bound by AGO4 and become trimmed by $3^{\prime}$ to 5 'exonucleases into sidRNAs which direct RdDM (Ye et al., 2016). In this study potential sidRNAs targetting TYLCV were found in both MM and Ty-1 plants, but their amounts were relatively low. To determine whether they present genuine sidRNAs, experiments with DCL knock out plants are needed to show they are still produced in the absence of DCL. Still, these pathways are less well characterised than the canonical RdDM pathway (for some nice overviews on canonical and non-canonical RdDM readers are referred to (Cuerda-Gil and Slotkin, 2016; Erdmann and Picard, 2020; Kenchanmane Raju et 
al., 2019)). Based on the data presented in this study, it is clear that Ty-1 enhances the production of 22 and 24-nt vsiRNAs, in which the 24-nt vsiRNAs are most likely involved in RdRM of the TYLCV genome. Whether the 22-nt vsiRNAs contribute to a stronger PTGS response and/or enhance a non-canonical RdDM pathway remains to be further investigated.

The increase in 22-nt and 24-nt vsiRNAs in Ty-1 plants is interesting in light of systemic silencing and tissue specificity of the virus. RNAi signals systemically spread via the phloem to other plant tissues, and both 22-nt and 24-nt sRNAs have been suggested to be able to move systemically (W., Chen et al., 2018; Molnar et al., 2010; Zhang et al., 2019). However, it still remains debated which molecule is transported (Zhang et al., 2019). Considering TYLCV is a virus that is phloem limited, i.e. replication primarily takes place there, it is tempting to speculate that vsiRNAs that are able to move systemically might be of higher importance to combat geminiviruses (Morilla et al., 2004; Rojas et al., 2001). It also raises an interesting question about the available RNAi machinery: Which elements of the RNAi machinery are expressed in phloem tissue and is Ty-1 also localized there? The expression of RNAi elements is not equal in all tissues, e.g. different AGO proteins show distinct tissue specificity (Havecker et al., 2010). Not much is known on the composition of the RNAi machinery (e.g. DCLs, AGOs, RDRs) in phloem tissue. Phloem tissue consists of several specialized cell type like phloem parenchyma cells, companion cells and sieve elements. The latter are elongated cells that interconnect into sieve tubes and present the conduits for phloem sap, but also for viruses and siRNAs to (systemically) disperse within a host. Although sieve elements are alive and contain organelles, RNAs and a proteome, they lack a nucleus and therefore may lack the RDR2-mediated TGS (canonical RdDM) pathway (Furuta et al., 2014). TYLCV DNA is mainly present in nuclei of phloem parenchymal and companion cells, although the DNA was also detected in the lumen of sieve tubes (Morilla et al., 2004). The sieve tube localization resembles most likely virions which are transported (Morilla et al., 2004), since the virus depends on the nuclear cellular DNA replication machinery to replicate (Gutierrez, 2000; Saunders et al., 1992). So, TGS activity will be found mainly in phloem parenchymal and companion cells. Whether this can move from neighbouring companion cells to sieve tubes and can exert any action there is not known. 
Although Ty-1 presents an atypical (non-NBS-LRR) resistance gene and does not lead to viral clearance, i.e. low titers of virus can still be detected, TYLCV isolates able to "break" Ty-1 resistance have been found. One of the best studied ones is TYLCV-IS76, resulting from a recombination event within the IR between two parental strains, TYLCV and TYLCSV. Under experimental conditions this recombinant showed to have a selective advantage compared to the parental viruses in Ty-1 tomato (Belabess et al., 2016). Recently it was proposed that this selective advantage relates to intragenomic interactions, since in vitro generated recombinants derived from other strains and virus recombination at a slightly different breakpoint (IS141) also exhibit a higher fitness than the parental viruses (Urbino et al., 2020). Within this study increased amounts of vsiRNAs were found in Ty-1 tomato, that mapped to the region of the recombination event. However, this region did not exhibit an increased level of cytosine methylation during an earlier study (Butterbach et al., 2014). Whether and how the vsiRNAs mapping to this region play a role in the resistance mechanism of Ty-1 and whether antiviral targeting of this (important) region acts as a driver for recombination to generate resistance breaking strains like TYLCV-IS76 remains to be further investigated.

In summary, the data presented in this study show that Ty-1 changes the antiviral siRNA profile of TYLCV and supports earlier observations that Ty-1 functions in enhancing the RNAi response. How and where Ty-1 functions to produce dsRNAs and which DLCs and AGOs are subsequently involved in the downstream RNAi will be one of the challenges for the future.

\section{Experimental Procedures}

\section{Plant material and virus stock}

During this study S. lycopersicum plants were maintained in a greenhouse at $23^{\circ} \mathrm{C}$ during the day and at $21{ }^{\circ} \mathrm{C}$ at night ( $16 \mathrm{~h}$ light/ $8 \mathrm{~h}$ dark regime) and a relative humidity of $60 \%$. S. lycopersicum cv. Moneymaker (MM) was used as susceptible control and a Ty-1 introgression line was derived from S. chilense LA1969 (Verlaan et al., 2013). Plants were infected with TYLCV via agroinoculation with an infectious 
clone of the TYLCV Israel strain isolated from Almeria, Spain, as described by Morilla et al. (2005) (GenBank AJ489258.1) or with untransformed agrobacteria as control (strain LBA4404).

\section{Agroinoculation}

The protocol as described in Chapter 2 was followed to perform the agrobacterium transient transformation assays, which is a slightly modified protocol of Bucher et al. (2003). In brief, A. tumefaciens was grown overnight at $28^{\circ} \mathrm{C}$ in $3 \mathrm{ml} \mathrm{LB3} \mathrm{(10}$ gL-1 trypton, 5 gL-1 yeast, 4 gL-1 NaCl, 1 gL-1 KCl, 3 gL-1 MgSO4.2H2O) medium containing proper antibiotic selection pressure. From this culture, $600 \mu 1$ was incubated overnight in $3 \mathrm{ml}$ induction medium (10.5 gL-1 $\mathrm{K}_{2} \mathrm{HPO}_{4^{\prime}} 4.5 \mathrm{gL}-1 \mathrm{KH}_{2} \mathrm{PO}_{4^{\prime}} 1$ gL- $1\left(\mathrm{NH}_{4}\right) 2 \mathrm{SO}_{4}, 0.5 \mathrm{gL}-1$ sodium citrate. $2 \mathrm{H}_{2} \mathrm{O}, 1 \mathrm{mM} \mathrm{MgSO}_{4} .7 \mathrm{H}_{2} \mathrm{O}, 0,2 \%(\mathrm{w} / \mathrm{v})$ glucose, 0,5\% (v/v) glycerol, $50 \mu \mathrm{M}$ acetosyringone, $10 \mathrm{mM}$ 2-(N-Morpholino)ethanesulfonic acid ((MES), pH 5.6)). The next day bacteria were pelleted by centrifugation (15 min. $2670 \mathrm{~g}$ ) and resuspended in MS MES buffer (Murashige and Skoog medium (Duchefa biochemie) supplemented with $150 \mu \mathrm{M}$ acetosyringone, $10 \mathrm{mM}$ MES and $87 \mathrm{mM}$ sucrose) at an OD600 of 0.5. The abaxial side of the first two true leaves of three week old tomato seedlings were infiltrated with the agrobacteria by pressure inoculation with a needle-less syringe.

\section{Nucleic acid purification}

First, systemically infected leaves (top leaves) were snap frozen in liquid nitrogen and stored at $-80^{\circ} \mathrm{C}$. RNA was isolated from this tissue using the mirVana miRNA isolation kit (Life technologies) according to the manufacturers protocol. In brief, upon grinding the leaf tissue in liquid nitrogen, the cells were lysed by adding lysis/ binding buffer in a 1:10 ratio $(\mathrm{w} / \mathrm{v})$ and mixing thoroughly. Subsequently, 1/10 volume of miRNA homogenate additive was added, followed by a 10 min incubation on ice. RNA was extracted by adding Acid-Phenol:Chloroform, mixing and spinning the sample. The aqueous phase was transferred to a new tube and $1 / 3$ volume of $100 \%$ ethanol was added. Upon mixing thoroughly, the sample was passed through a 
Filter Cartridge. From this point, both the filtrate, containing the sRNAs, as the filter cartridge, containing the RNA fraction depleted of sRNAs, was further processed. To the filtrate $2 / 3$ volume $100 \%$ ethanol was added and this mixture was passed through a second filter cartridge. This filter cartridge was washed once with miRNA wash solution 1 and subsequently washed twice with wash solution $2 / 3$. The sRNA fraction was eluted with MiliQ. The first filter cartridge containing the RNA fraction depleted of sRNAs was washed once with miRNA wash solution 1 and subsequently washed twice with wash solution 2/3. This RNA fraction was also eluted with MiliQ. The integrity of the RNA fractions was checked on a RNAse free agarose gel.

From the samples used for the RNA isolations, also DNA was isolated following the cetyltrimethyl ammonium bromide (CTAB) method of Doyle and Doyle (1987) with slight modifications as described by Fulton et al. (1995) (Chapter 2). In brief, grinded plant material was mixed with CTAB buffer (0.1 M TRIS, 0.7 M NaCl, 0.01 M EDTA, $2 \%$ CTAB) and subsequently incubated for 1 hour at $65^{\circ} \mathrm{C}$. After chloroform isoamyl alcohol extraction, the DNA was precipitated from the aqueous phase by adding isopropanol in a 1:1 ratio. DNA was pelleted, dried and dissolved in MilliQ. DNA and RNA concentrations were measured with a Nanodrop ND-1000 device.

\section{Library preparation and sequencing}

The sRNA fraction was analysed by capillary electrophoresis on a Shimadzu MultiNA microchip. The QIAseq miRNA library kit (Qiagen) was used according to the manufacturer's instructions to synthesize cDNA. In short, oligonucleotide adapters were ligated to the 5' and 3' ends of the RNA samples. First-strand cDNA was synthesized using M-MLV reverse transcriptase and the 3' adapter as primer. Amplification of the resulting cDNA was performed by PCR with a highfidelity DNA polymerase in 16 PCR cycles. The 3' sequencing adapters included a sample specific six nucleotide long barcode sequence. The cDNA was purified using the magnetic beads provided in the kit. The cDNA was analysed by capillary electrophoresis on a Shimadzu MultiNA microchip. The cDNA samples were pooled in approximately equimolar amounts and the size range of 160-200 bp was selected from a polyacrylamide gel. An aliquot of the size fractionated pool was analysed 
by capillary electrophoresis on a Shimadzu MultiNA microchip. The cDNA pool was sequenced on an Illumina NextSeq 500 system using 75 bp read length.

\section{Bioinformatics analysis of sRNA data}

The data analysis was performed on the Galaxy server (Afgan et al., 2018). Quality assessments were performed with the FastQC tool (Galaxy version 0.72+galaxy1). From each read the adapter sequences were removed using the clip tool (Galaxy Version 1.0.3+galaxy0). Sequence length was determined by using the compute sequence length tool (Galaxy Version 1.0.3) and the count tool (Galaxy Version 1.0.3). Reads were mapped to the tomato genome (solgenomics, SL4.0) using Bowtie2 (Galaxy Version 2.3.4.3+galaxy0, default settings) and read length of tomato aligned reads calculated with the compute sequence length tool (Galaxy Version 1.0.3) and the count tool (Galaxy Version 1.0.3). Reads were mapped to the viral genome (TYLCV, GenBank AJ489258.1) using Bowtie2 (Galaxy Version 2.3.4.3+galaxy0, allowing 1 mismatch). From the bowtie output, genome profiles were generated by using the count (Galaxy Version 1.0.3), filter (Galaxy Version 1.1.1), join two datasets (Galaxy Version 2.1.3), compute (Galaxy Version 1.3.1) and advanced cut (Galaxy Version 1.1.0) tools. To determine the $5^{\prime}$ end nucleotides, the sequence logo tool (Galaxy Version 3.5.0) was used.

\section{qPCR for titer determination}

Relative virus titers of TYLCV was determined by qPCR using actin (Solyc04g011500) as internal control. The reaction mixture contained 1x SybrSelect (Applied Biosystems), $300 \mathrm{nM}$ forward primer, $300 \mathrm{nM}$ reverse primer and $10 \mathrm{ng}$ genomic DNA. The primers TYLCV-F (5'-TTCGTCTAGATATTCCCTATATGAGGAGGTA-3') and TYLCV-R (5'-GGGAAGCCCATTCAAATTAAAGG-3') (Powell et al., 2012) were used to amplify TYLCV, and actin-F (5'-GAAATAGCATAAGATGGCAGACG-3') and actin-R (5'-ATACCCACCATCACACCAGTAT-3') to amplify actin. The qPCR was performed in an Applied Biosystems AB7500, using the following cycling conditions: 2 min $95^{\circ} \mathrm{C}$, 40 cycles of $15 \mathrm{sec} 95^{\circ} \mathrm{C}$ and $1 \mathrm{~min} 60^{\circ} \mathrm{C}$, followed by a melting curve with $0.5^{\circ} \mathrm{C}$ 
steps from $60{ }^{\circ} \mathrm{C}$ to $95{ }^{\circ} \mathrm{C}$ to determine PCR specificity. Relative viral titers were calculated using the DeltaDeltaCt method (Livak and Schmittgen, 2001). Values were normalized relative to the internal control and calibrated to levels of the TYLCV infected MM plants, which were set as 1.

\section{Acknowledgements}

The authors would like to thank Prof. Eduardo Rodríguez Bejarano (Universidad de Málaga, Malaga, Spain) for providing the infectious TYLCV (Almeria isolate) clone, and prof. Monique van Oers (Wageningen University) is acknowledged for critically reading and discussion. This research was financially supported by NWO-CNPq within the Joint Research Project Biobased Economy (729.004.011). The authors declare no conflicts of interest. 


\section{Supplemental data}
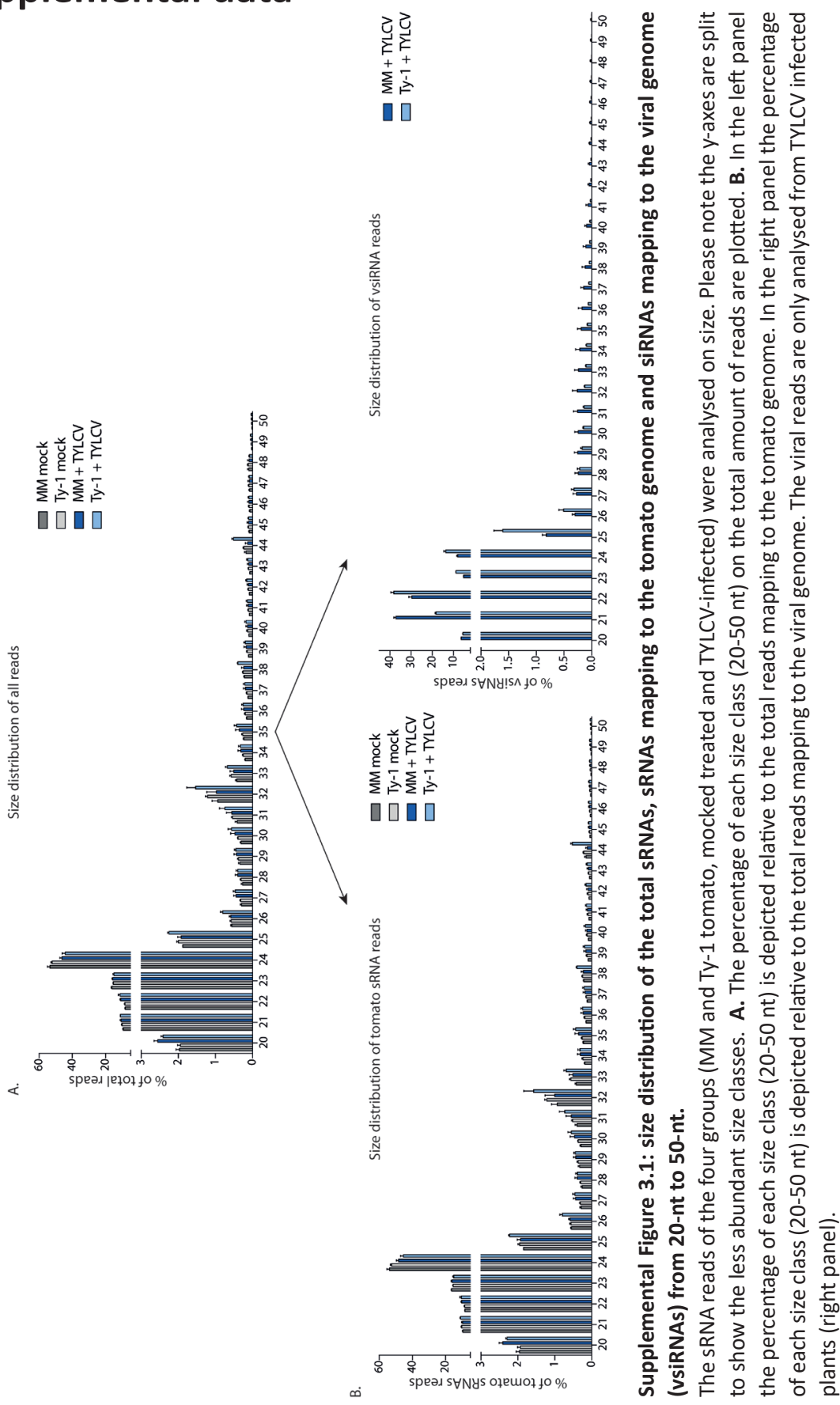


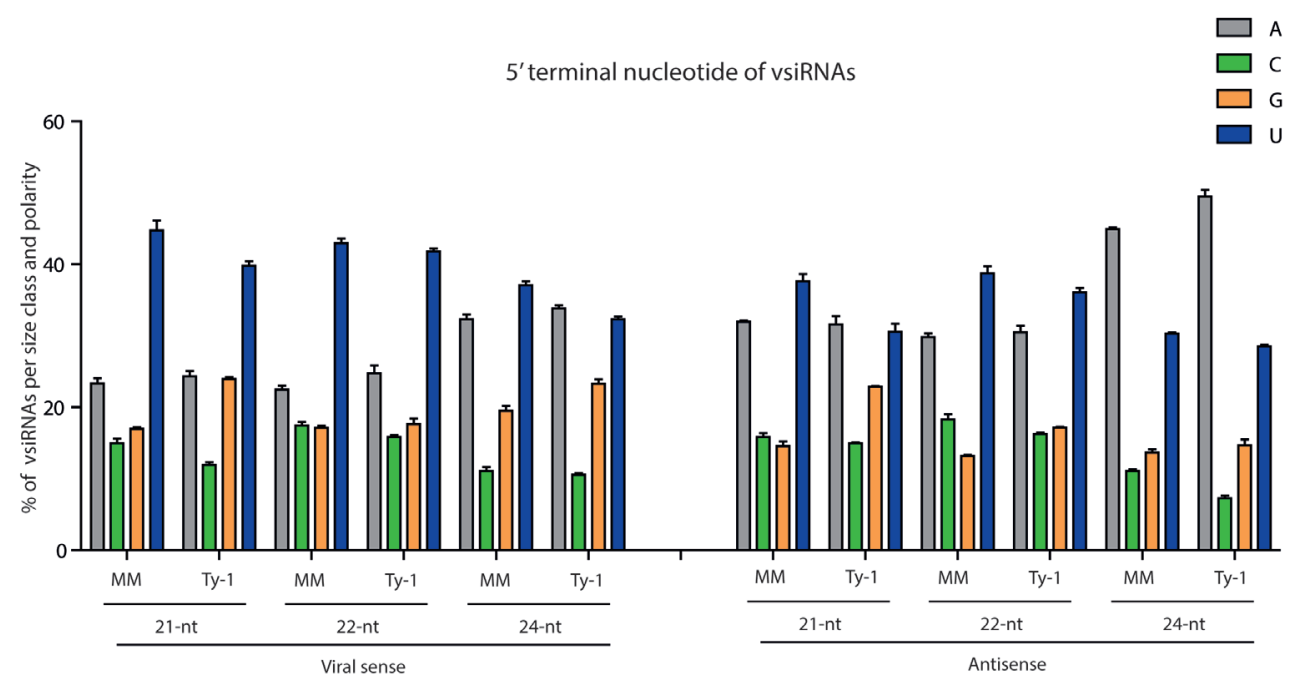

Supplemental Figure 3.2: Distribution of the $5^{\prime}$ terminal nucleotide of sense and antisense vsiRNA reads from MM and Ty-1 tomato.

Viral siRNAs isolated from TYLCV infected MM or Ty-1 tomato, depicted per size class of 21-nt, 22-nt and 24-nt, and polarity, and showing the percentage of reads containing adenine (A), cytosine (C), guanine $(\mathrm{G})$, or uracil (U) as 5 'terminal nucleotide. The bar represents the average and standard deviation of three biological replicates. 
A.
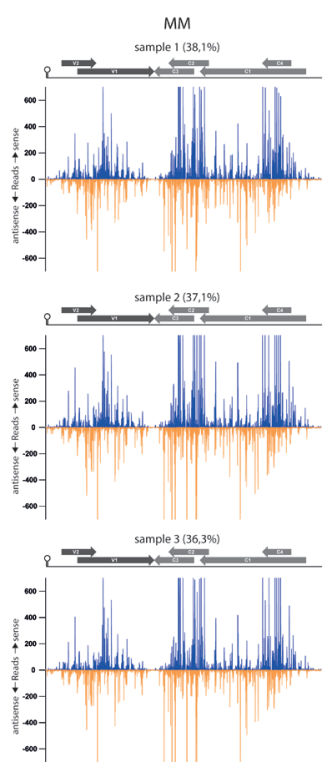

C. 21-nt vsiRNAs

24-nt vsiRNAs
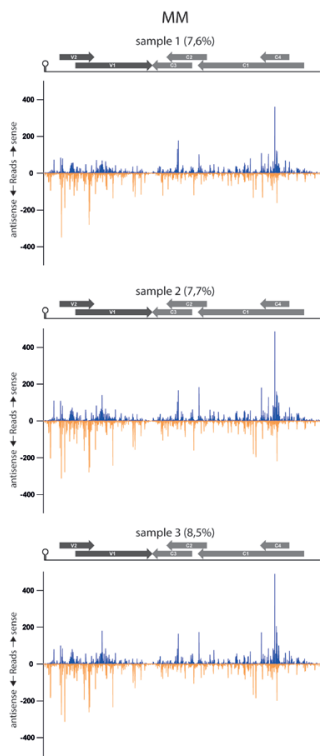
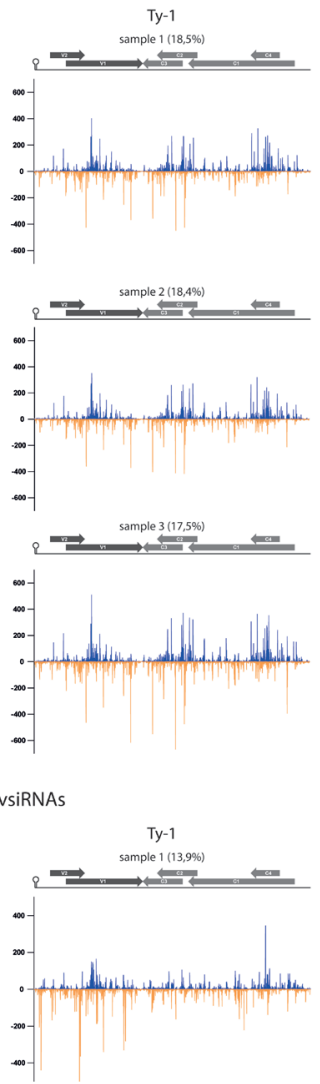

B.
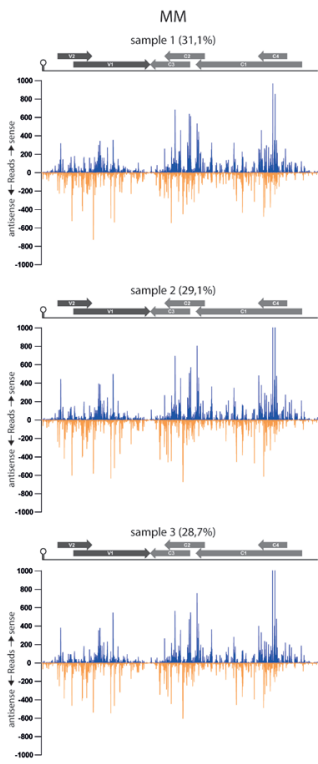

D.
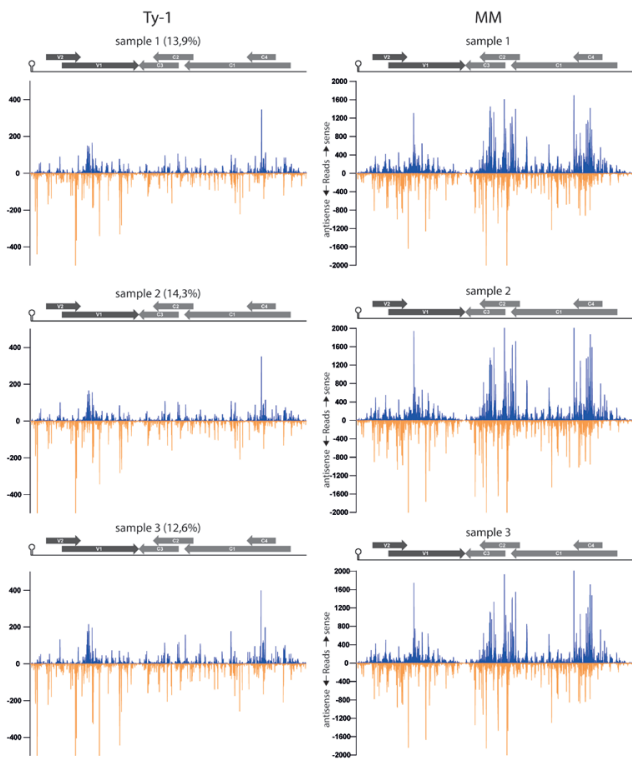

22-nt vsiRNAs
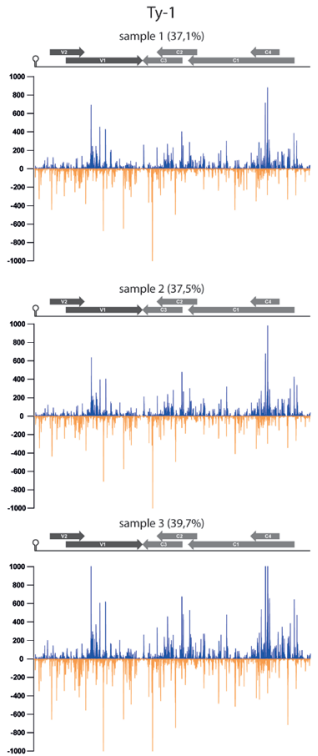

all vsiRNAs
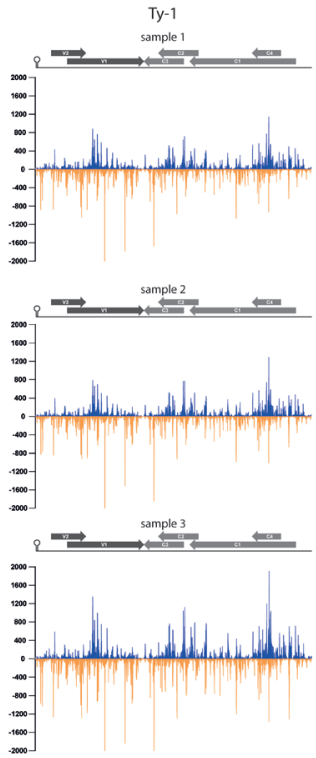

Supplemental Figure 3.3: The distribution of vsiRNAs on the TYLCV genome in three biological replicates of MM and Ty-1 plants.

Viral siRNAs isolated from infected MM (left panel) or Ty-1 bearing (right panel) tomato were mapped on the genome of tomato yellow leaf curl virus (TYLCV). The number of reads at each nucleotide position of the TYLCV genome is plotted for each sample (three biological replicates). Blue bars represent sense reads 
starting at each respective nucleotide, while the orange bars represent antisense reads ending at that position. The depicted percentage in the title represents the proportion that belongs to the size class in that specific sample relative to all vsiRNAs. A schematic overview of the TYLCV genome is depicted above the graphs, with the viral ORFs indicated as grey arrows and the intergenic region at the left and right side. The vsiRNA distribution profile is depicted for $\mathbf{A}$. the 21-nt size-class, B. the 22-nt size-class, $\mathbf{C}$. the 24-nt size-class and D. all vsiRNAs. 
MM

21-nt vsiRNAs $(37,2 \%)$

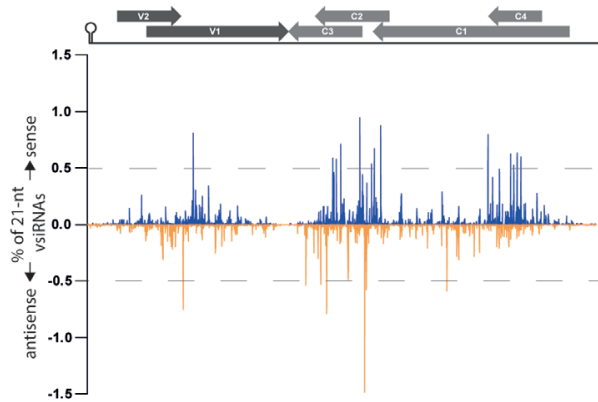

22-nt vsiRNAs $(29,7 \%)$
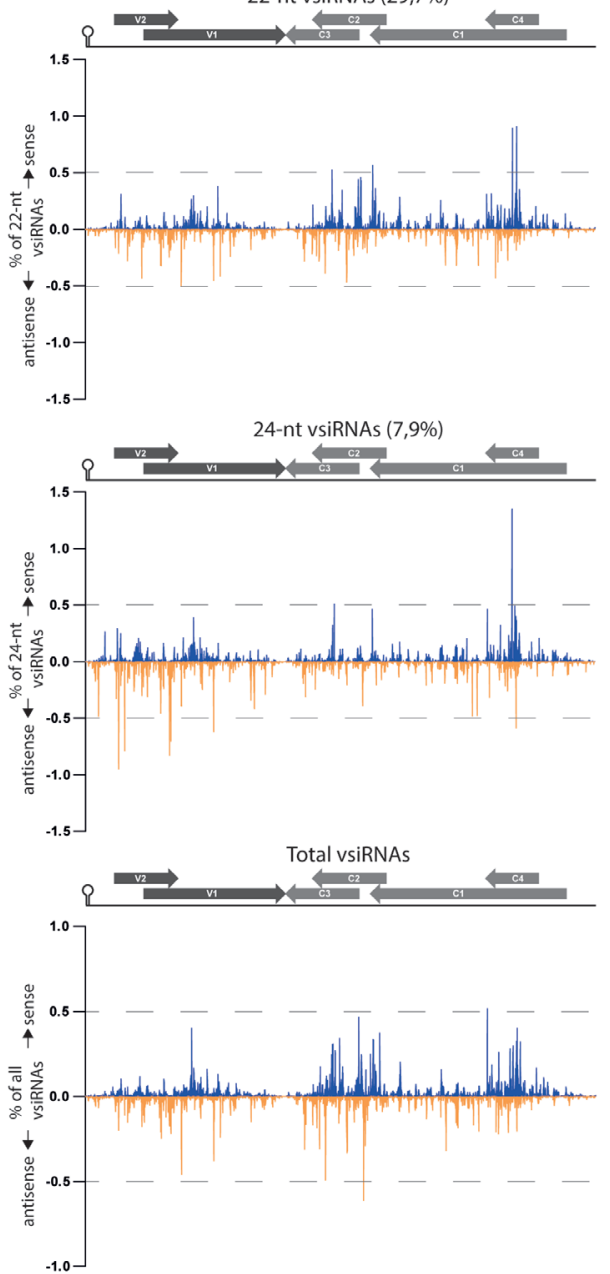

Ty-1

21-nt vsiRNAs $(18,1 \%)$

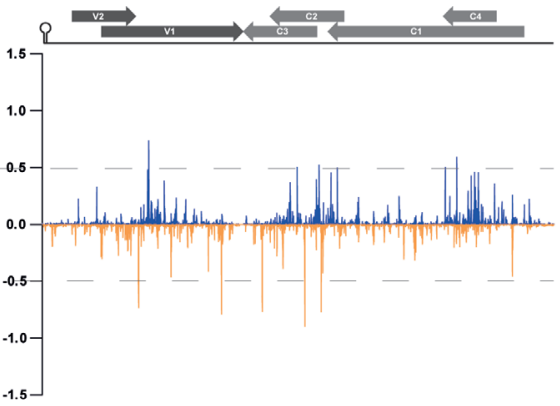

22-nt vsiRNAs $(38,3 \%)$
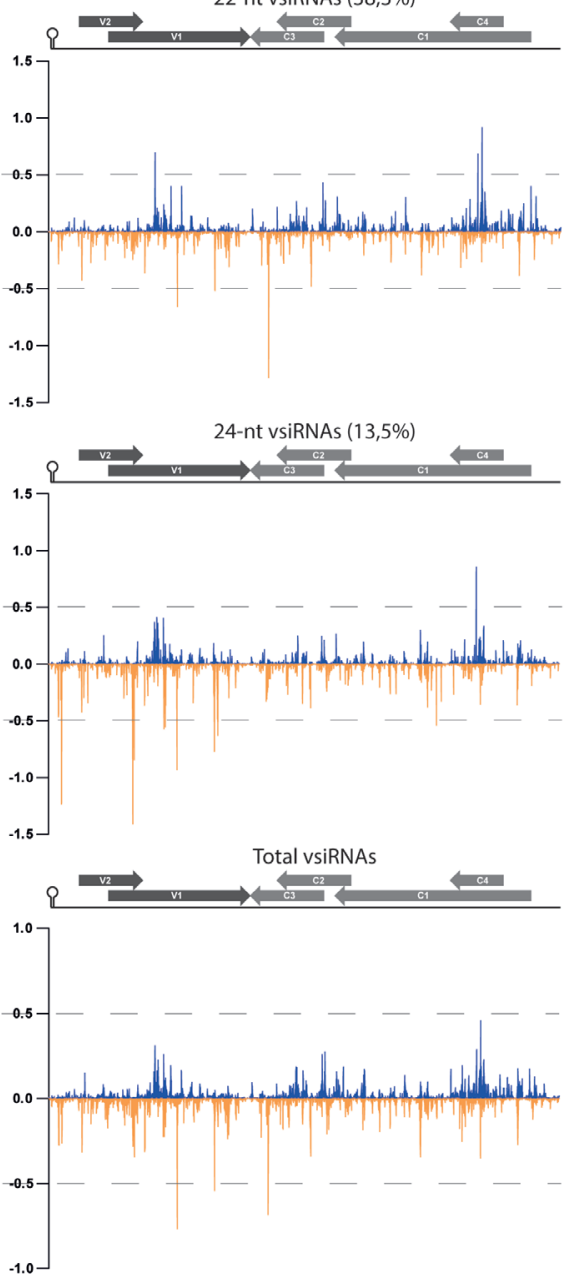
Supplemental Figure 3.4: Genomic distribution of vsiRNAs, depicted as percentage of the total vsiRNAs of that size class at each nucleotide position in MM and Ty-1 tomato.

Viral siRNAs isolated from infected MM (left panel) or Ty-1 bearing (right panel) tomato are mapped on the genome of tomato yellow leaf curl virus (TYLCV). Per size class, the vsiRNAs are depicted as percentage of the total reads at each nucleotide position of the TYLCV genome (average from three biological replicates). Blue bars represent sense reads starting at each respective nucleotide, while the orange bars represent antisense reads ending at that position. The percentage in the title represents the proportion that belongs to the size class of all vsiRNAs in either MM or Ty-1 plants. A schematic overview of the TYLCV genome is presented at the top of each graph, with the viral ORFs indicated as grey arrows and the intergenic region at the left and right side. 
A.

21-nt vsiRNAs
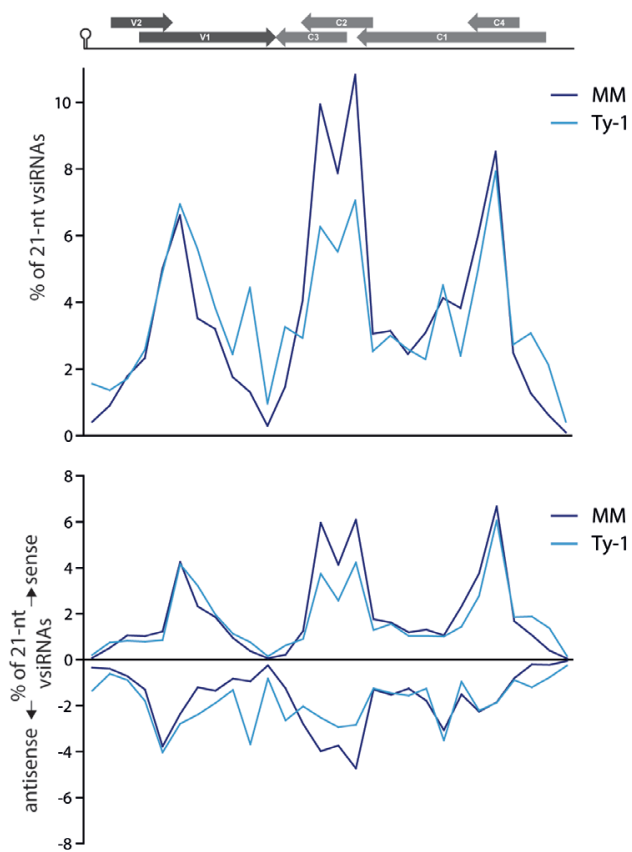

B.
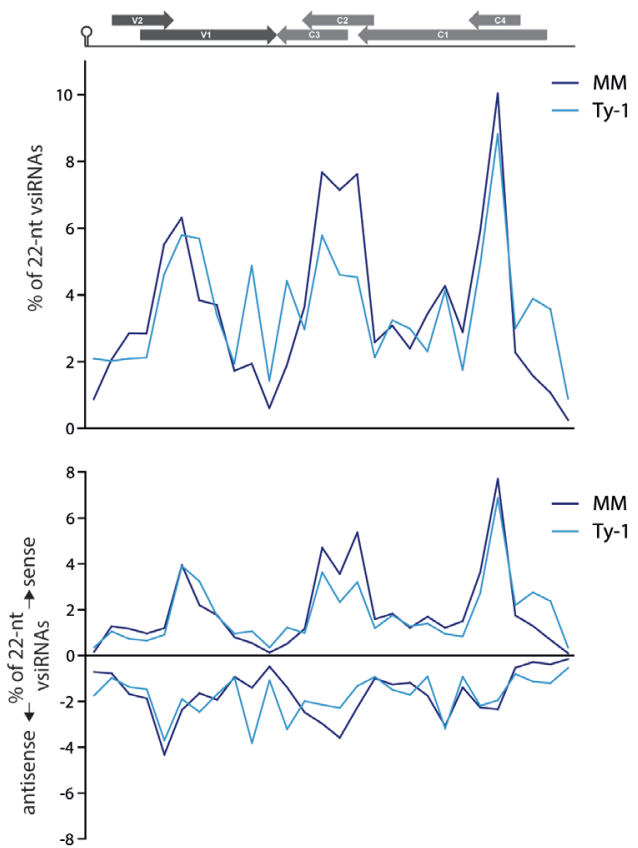

C.

24-nt vsiRNAs
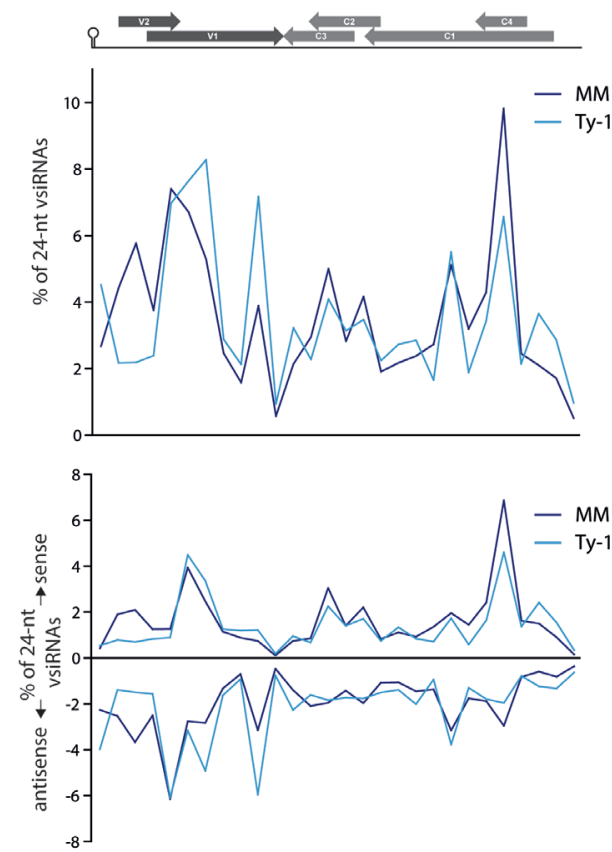

D.
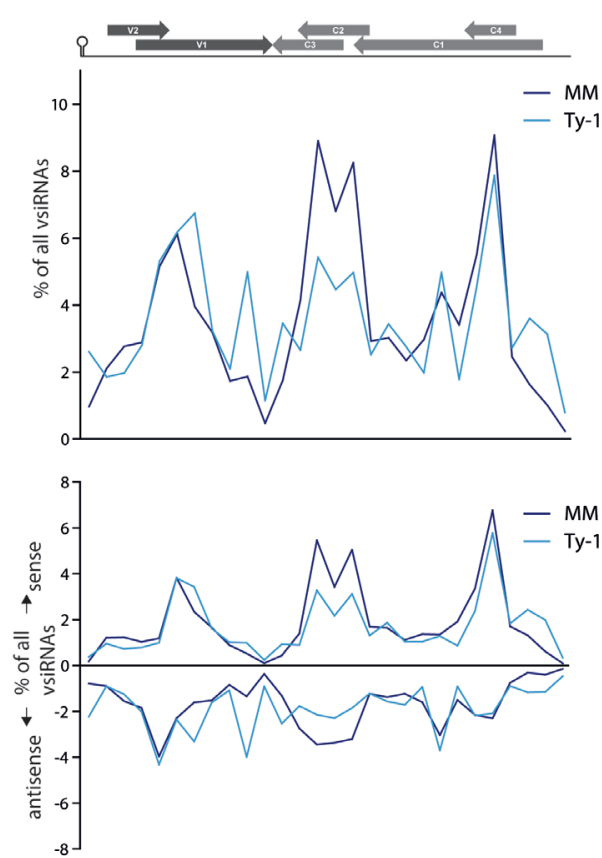


\section{Supplemental Figure 3.5: Percentage of vsiRNA reads per 100 nucleotides in the TYLCV genome.}

Viral siRNAs isolated from infected MM (dark blue line) or Ty-1 bearing (light blue line) tomato are mapped on the genome of tomato yellow leaf curl virus (TYLCV). The percentages of vsiRNA reads per 100 nucleotides of the TYLCV genome (amount of reads for position 1-100, 101-200 etc.) relative to the total amount of viral reads of the depicted size class are plotted. The graphs represent the average of three biological replicates. From each set of panels, the upper panel presents the sense and antisense polarities taken together, while the lower panel presents both polarities specified. A schematic overview of TYLCV genome is depicted at the top of each graph, with the viral ORFs indicated as grey arrows and the intergenic region at the left and right side. The genome distribution is depicted for A. 21-nt, B. 22-nt, C. 24-nt and D. all vsiRNAs. 
A.

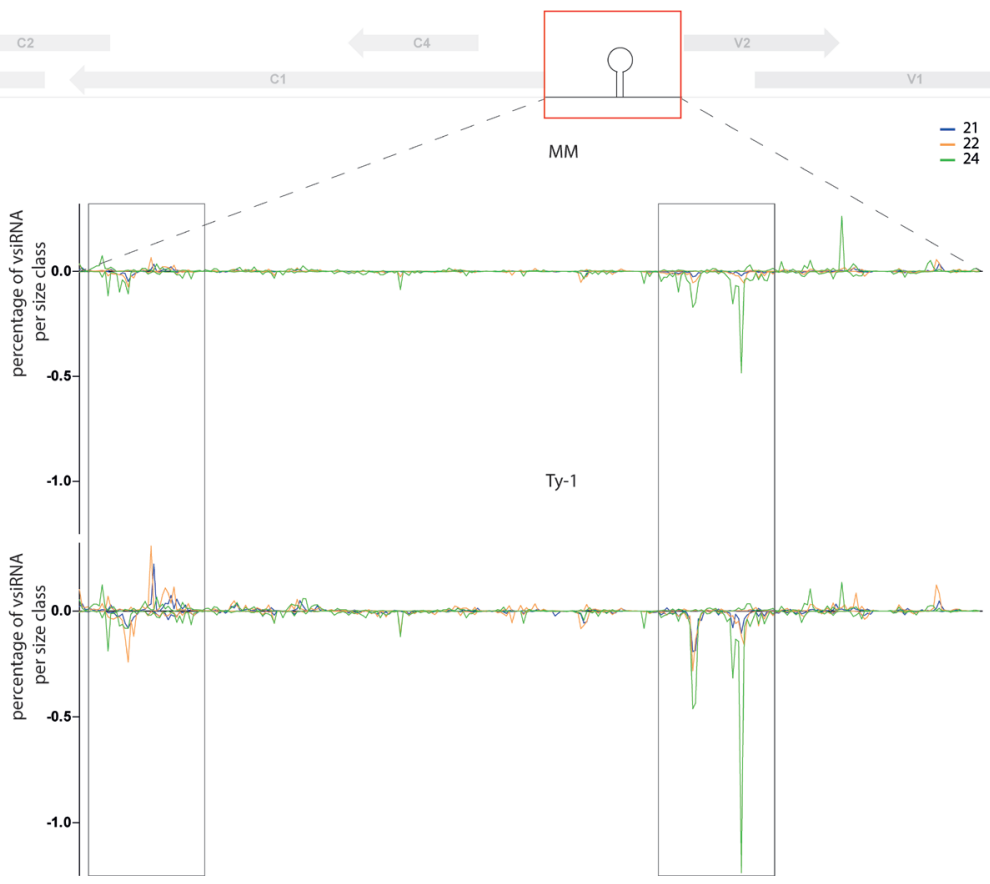

B.

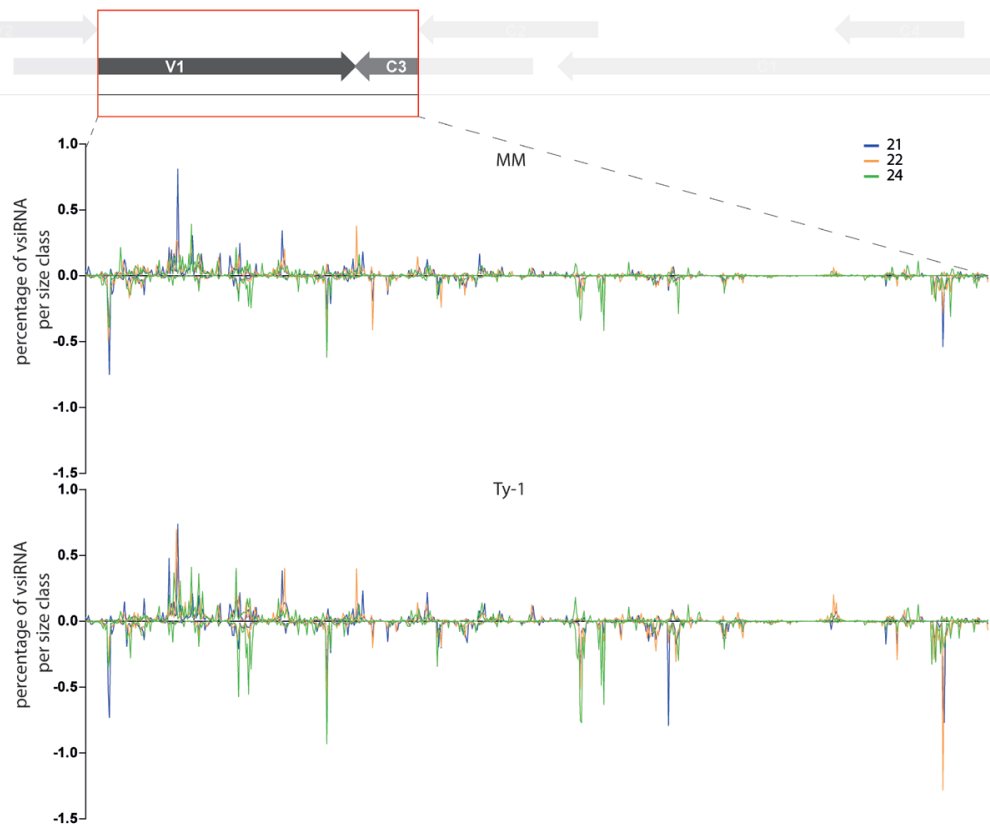


Supplemental Figure 3.6: Close-up on the vsiRNA distribution in the intergenic region and "V1-C3 junction", depicted as percentages of the total amount of vsiRNA reads at each nucleotide position of the TYLCV genome.

Viral siRNAs isolated from infected MM (top panel) or Ty-1 bearing (lower panel) tomato are mapped on the genome of tomato yellow leaf curl virus (TYLCV). The amounts of vsiRNA reads at each nucleotide position of the TYLCV genome are presented as percentage from the total amount of reads of the depicted size class (average of three biological replicates). Positive bars represent sense reads starting at each respective nucleotide, while the negative bars represent antisense reads ending at that position. A schematic overview of TYLCV genome is depicted at the top of each graph, with the viral ORFs indicated as grey arrows and the region zoomed in highlighted with a red box. A. Genomic distribution of vsiRNAs in the intergenic region (nucleotides 2623-154). Major differences between MM and Ty-1 tomato are highlighted with black boxes.

B. Genomic distribution of vsiRNAs in the "V1-C3 junction" (nucleotides 506-1232). 
A.

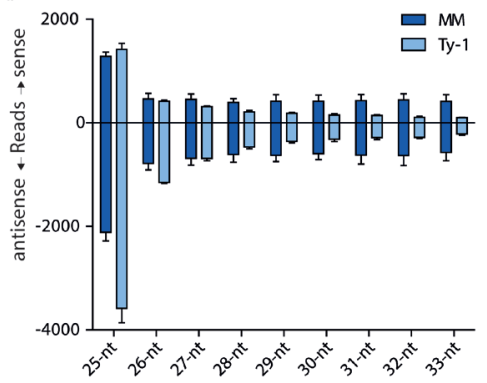

B.

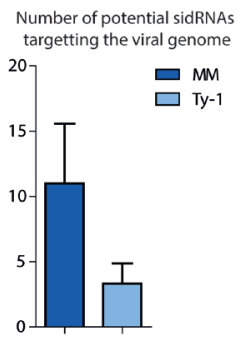

C. Potential sidRNA (nt 1324 start position in MM samples) AGGAAGGTAATGGGGATTCAC AGGAAGGTAATGGGGATTCACA AGGAAGGTAATGGGGATTCACAA AGGAAGGTAATGGGGATTCACAAA AGGAAGGTAATGGGGATTCACAAAT AGGAAGGTAATGGGGATTCACAAAT AGGAAGGTAATGGGGATTCACAAATG AGGAAGGTAATGGGGATTCACAAATGT AGGAAGGTAATGGGGATTCACAAATGTTT AGGAAGGTAATGGGGATTCACAAATGITIT AGGAAGGTAATGGGGATTCACAAATGITTTC AGGAAGGTAATGGGGATTCACAAATGTTTTCT AGGAAGGTAATGGGGATTCACAAATGTITTCTC AGGAAGGTAATGGGGATTCACAAATGITTTCTCA AGGAAGGTAATGGGGATTCACAAATGTTTTCTCAA AGGAAGGTAATGGGGATTCACAAATGTTTTCTCAAC AGGAAGGTAATGGGGATTCACAAATGITTCTCAACT

D.
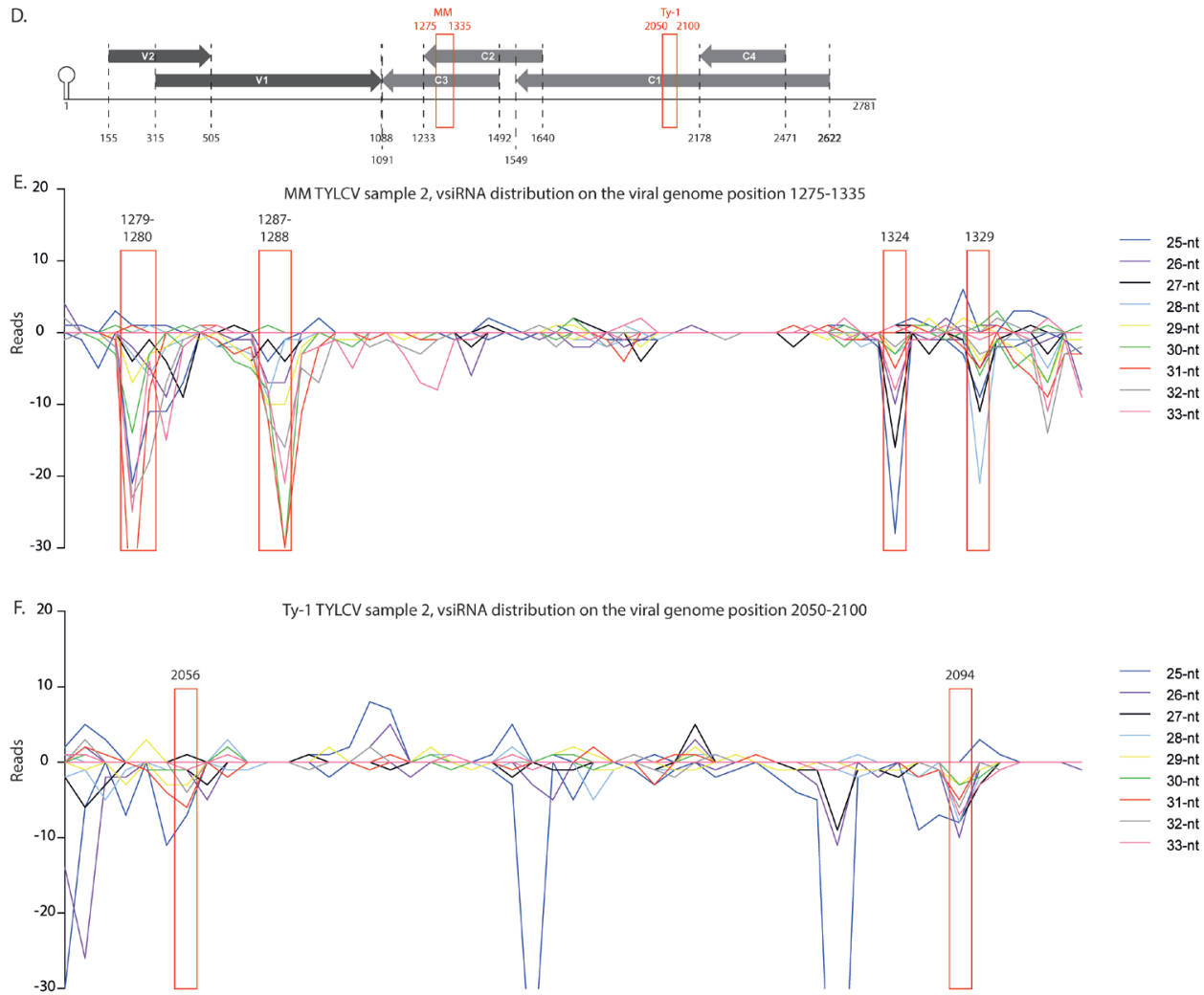

Supplemental Figure 3.7: Potential viral sidRNAs generated during TYLCV-infection of MM and Ty-1 tomato. Viral siRNAs isolated from TYLCV-infected MM or Ty-1 bearing tomato, were sequenced and subsequently mapped on the viral genome. A. Graphical presentation of the amount of sense and antisense orientated viral reads within the size range of 25-33 nt from MM and Ty-1 tomato. The average of three biological replicates is depicted with the standard deviation. B. Graph presenting the number of potential viral sidRNAs in MM and Ty-1 tomato (average of three biological replicates with standard deviation). Potential viral sidRNAs were identified from spots in the TYLCV genome that are targeted by vsiRNAs from different size classes (25$33 \mathrm{nt}$ ) and sharing the same 5'end. C. Potential viral sidRNAs identified in MM tomato and mapping to the TYLCV genome at position $1324 \mathrm{nt}$. D. Schematical diagram of the TYLCV genome and location of potential 
sidRNAs highligthed in red boxes (Sup. Table 3.1). E. Close-up of the distribution of vsiRNAs (size range 25-33 $\mathrm{nt}$ ) on the TYLCV genome region 1275-1335 nt in MM biological replicate sample 2. The amount of reads starting with the 5' end of the vsiRNAs at each nucleotide position of the TYLCV genome is plotted, in either sense or antisense orientation. With a red box the potential sidRNAs (in size range 25-33 nt) are indicated. F. Close-up of the distribution of vsiRNAs (size range 25-33 nt) on the TYLCV genome region 2050-2100 nt in Ty-1 biological replicate sample 2. The amount of reads starting with the $5^{\prime}$ end of the vsiRNA at each nucleotide position of the TYLCV genome is plotted, in either sense or antisense orientation. With a red box the potential sidRNAs (in size range 25-33 nt) are indicated.

Supplemental Table 3.1: Identification of potential sidRNAs and their starting position on the TYLCV genome (1-2781).

\begin{tabular}{|c|c|c|c|c|c|}
\hline \multicolumn{2}{|c|}{ MM TYLCV 1} & \multicolumn{2}{|c|}{ MM TYLCV 2} & \multicolumn{2}{|c|}{ MM TYLCV 3} \\
\hline \multicolumn{2}{|c|}{$(+)$} & \multicolumn{2}{|c|}{$(+) \quad(-)$} & \multicolumn{2}{|l|}{$(+)$} \\
\hline & 1262 & \multirow{12}{*}{$\begin{array}{l}575 \\
694 \\
883\end{array}$} & 1258 & \multirow[t]{12}{*}{575} & 1230 \\
\hline & 1279 & & 1262 & & 1279 \\
\hline & 1288 & & 1279 & & 1287 \\
\hline & 1324 & & 1280 & & 1324 \\
\hline & 1329 & & 1287 & & 1329 \\
\hline & \multirow[t]{7}{*}{1332} & & 1288 & & 1333 \\
\hline & & & 1324 & & 1567 \\
\hline & & & 1329 & & 1649 \\
\hline & & & 1530 & & 1796 \\
\hline & & & 1558 & & 2001 \\
\hline & & & 1796 & & 2347 \\
\hline & & & 2162 & & \\
\hline \multicolumn{2}{|c|}{ Ty-1 TYLCV 1} & \multicolumn{2}{|c|}{ Ty-1 TYLCV 2} & \multicolumn{2}{|c|}{ Ty-1 TYLCV 3} \\
\hline$(+)$ & $(-)$ & & $(-)$ & $(+)$ & $(-)$ \\
\hline \multirow[t]{4}{*}{627} & 2094 & \multirow[t]{4}{*}{627} & 929 & & 495 \\
\hline & & & 2056 & & 930 \\
\hline & & & 2094 & & 2094 \\
\hline & & & 2750 & & \\
\hline
\end{tabular}

Overview of potential sidRNA and their position in the TYLCV genome (nucleotide number 1-2781, sense or antisense orientation). Highlighted in grey are the spots with potential sidRNAs that are found in all three biological replicates. 
a nu Bun -

1)

5s

त) की

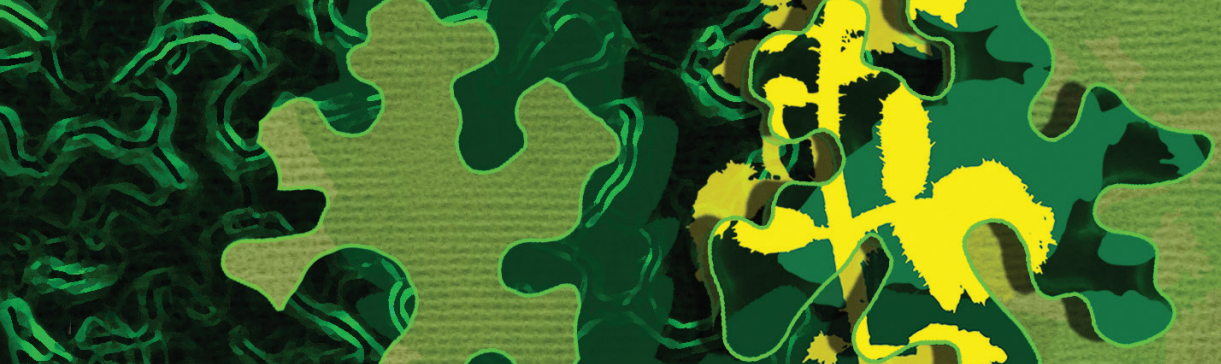

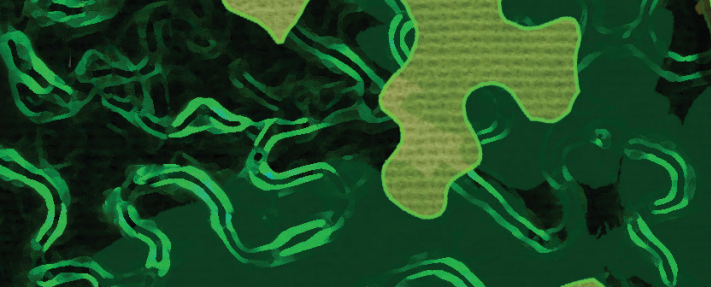

sis (2) at 2 ?

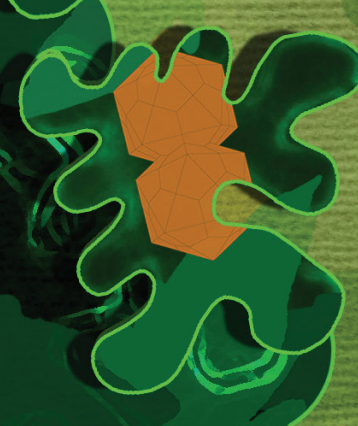

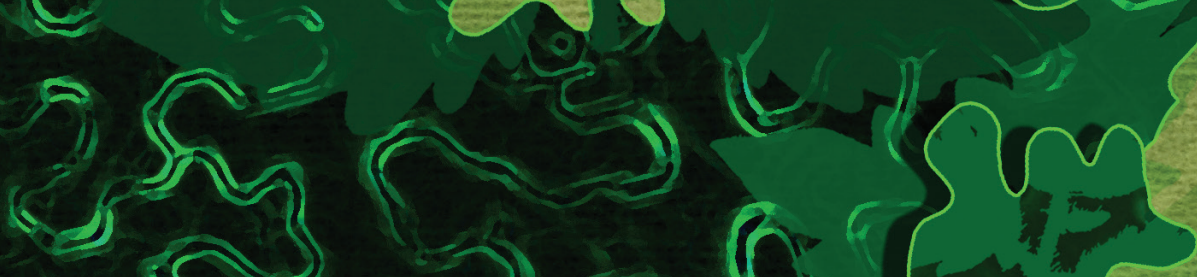

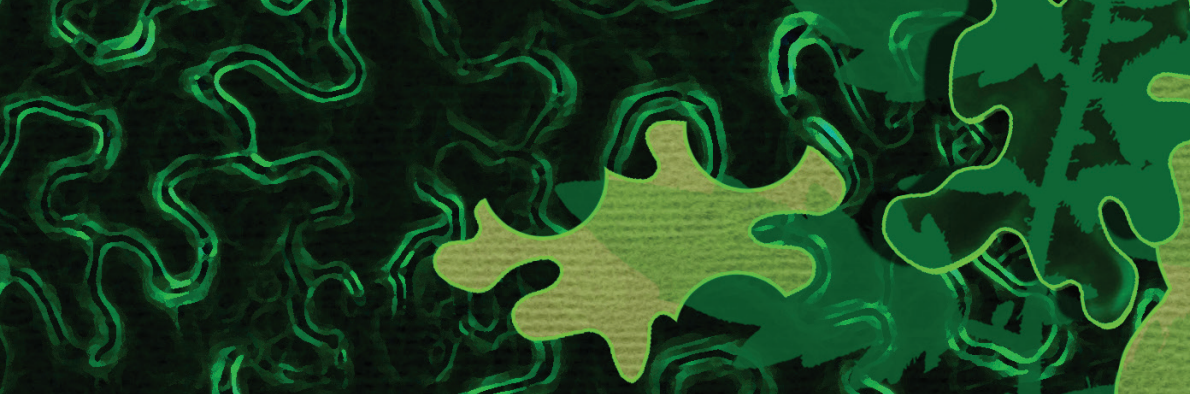
(B) $) \sqrt{0}$ (2) 


\section{Chapter 4}

\section{Analysis of cytoplasmic RNA Processing bodies in anti-viral RNA interference of Tomato Yellow Leaf Curl geminivirus}

C.M. Voorburg, N. Sosef, R. Kormelink 


\section{Summary}

Earlier studies have identified proteins from the host RNA quality control system localized in RNA Processing bodies (P-bodies) e.g., Exoribonuclease 4 (XRN4) and Decapping 2 (DCP2), as suppressors of the cytoplasmic post-transcriptional gene silencing (PTGS) pathway in plants. Plants impaired in these genes exhibit enhanced PTGS against RNA viruses and indicate that the host RNA quality control system competes for (aberrant) RNA substrate with RNA-dependent RNA polymerases (RDRs) from the RNA interference (RNAi) pathway. Here we have investigated whether impairing elements of the (cytoplasmic) RNA quality control system also affects the antiviral RNAi response to Tomato yellow leaf curl virus (TYLCV), a nuclear replicating DNA virus. Nicotiana benthamiana ( $N$. benthamiana) plants VIGS-silenced on RNA quality control elements DCP2, DCP5 or VCS showed enhanced levels of PTGS during transgene GFP expression. However, no change in susceptibility of the plants against TYLCV was observed. Viral titers determined in RNA quality control-compromised $N$. benthamiana were similar to those in wild type plants and indicated that the RNA quality control system / P-bodies do not compete for RNA substrate with RDRs involved in mounting a strong RNAi response towards TYLCV. This finding is discussed in light of the recent cloning and characterization of the $T y$ - 1 resistance gene, a member of the $\operatorname{RDR} \gamma(\operatorname{RDR} 3,4,5)$ class and involved in mounting an enhanced TGS response against TYLCV. 


\section{Introduction}

One of the most important defence responses in plants against viruses relies on RNA interference (RNAi), a highly conserved mechanism occurring in all eukaryotes and comprising two branches (Ding and Voinnet, 2007). The first one targets viral RNA molecules from RNA and DNA viruses and the second one the genome of DNA viruses, referred to as Post Transcriptional Gene Silencing (PTGS) and Transcriptional Gene Silencing (TGS) respectively (Mallory and Vaucheret, 2010). Whereas PTGS leads to the degradation of target RNA molecules in the cytoplasm, TGS inhibits transcription of target DNA molecules and occurs in the nucleus. The trigger for RNAi is the formation of dsRNA, and during a viral infection originates from replicative intermediates or RNA folding structures. Dicer Like proteins (DCL) cleave the dsRNA into small interfering RNAs (siRNAs) of 21-24 nucleotide (nt) in size (Hammond, 2005). In plants four DCLs exist i.e., DCL1 to 4. During PTGS, dsRNA is primarily cleaved by DCL4 (alternatively DCL2) into 21-22 nt siRNAs. After the siRNA is unwound, one (guide) strand is uploaded into a RNA-Induced Silencing Complex (RISC) that contains an Argonaute (AGO) protein as the main core component. This activated RISC complex subsequently is able to sense RNA molecules with sequence complementarity to the guide strand and cleaves the target RNA molecule leading to its degradation (Mallory and Vaucheret, 2010). Cleavage relies on the slicing activity of the AGO1 core component. In plants cleaved, aberrant RNA molecules either enter the cytoplasmic RNA quality control (RQC) system for further degradation or they will be used as a substrate by RNA-Dependent RNA polymerase (RDR) 6 for further amplification of the RNAi response (Tsuzuki et al., 2017). RDR6, together with co-factor SUPPRESSOR OF GENE SILENCING 3 (SGS3), converts the aberrant RNA into dsRNA and thereby increases the amount of viral dsRNA that enters the PTGS pathway. The amplification takes place in (cytoplasmic) siRNA bodies and is important to mount a strong antiviral RNAi response (Kumakura et al., 2009). Knock out (KO) of RDR6 leads to hypersusceptibility against RNA viruses, but not to DNA viruses (Raja et al., 2008; Schwach et al., 2005; Wang et al., 2010). The TGS pathway occurs in the nucleus, where it targets (nuclear replicating) DNA viruses like geminiviruses. During TGS dsRNA molecules are processed by DCL3 into $24 \mathrm{nt}$ siRNAs, from which one strand is uploaded into RISC. This complex, instead of AGO1, now contains AGO4 and enables RISC to surveil for the presence of DNA target 
molecules with sequence complementarity to the siRNA strand, and methylate cytosine residues within the DNA strand. As a result gene expression is silenced at the transcriptional level (Mallory and Vaucheret, 2010).

As a counter defence, plant viruses have evolved mechanisms to inhibit the antiviral RNAi response. The most common way is by encoding proteins, named RNA Silencing Suppressors (RSS), that interfere with several steps of the RNAi response. One way by which RSS proteins can inhibit PTGS is to bind dsRNA and siRNAs which prevents cleavage by DCL and uploading into the RISC complex respectively. An example of such RSS protein is the Tomato Spotted Wilt Virus encoded protein NSs (Schnettler et al., 2010). Also the slicing activity of AGO can be inhibited. For example cucumber mosaic virus $2 b$ protein inhibits the slicing activity of AGO via a direct interaction, while the polerovirus encoded protein PO destabilizes AGO (Baumberger et al., 2007; Zhang et al., 2006). RSS proteins can also inhibit TGS. Interaction with AGO4 can inhibit methylation, which is the working mechanism of the geminiviral protein V2 (Wang et al., 2019). Most TGS inhibiting proteins target different steps of the methylation pathway. For example the geminiviral proteins C1 together with C4 decreases the expression of two different methyltransferases (Rodríguez-Negrete et al., 2013). The RSS activity is often essential to establish a successful viral infection in the plant.

Plants also encode proteins that inhibit the PTGS response. Most of these endogenous RSS proteins are involved in RQC pathways. RQC ensures elimination of defective messenger RNAs (mRNA) which encode potential toxic proteins and regulates protein abundance by mRNA turnover. Degradation of functional mRNAs is prevented by a 5'cap structure and a 3'poly A tail. mRNAs lacking one of these features are degraded by a complex of enzymes (tabno et al., 2016). In addition, defective mRNAs containing premature stop-codons are recognized by the nonsense mediated decay (NMD) pathway and are subsequently decapped or deadenylated and will be degraded via exonucleolytic cleavage (Shoemaker and Green, 2012). Upon deadenylation, $3^{\prime}$ to $5^{\prime}$ exonucleolytic degradation takes place by the exosome. $5^{\prime}$ to $3^{\prime}$ degradation takes place in the cytoplasmic RNA Processing bodies (P-bodies) (Łabno et al., 2016). First, Decapping (DCP) 2 in a complex with DCP1, DCP5 and Varicose (VCS) removes the Cap structure. Subsequently, the exoribonuclease XRN4 
degrades the RNA molecule. P-Bodies localize adjacent to siRNA bodies (De Alba et al., 2015; Kumakura et al., 2009).

Mutations in the NMD pathway and exosomes enhance transgene silencing by PTGS, and this effect can be abolished in a RDR6 mutant background (Moreno et al., 2013; Yu et al., 2015; Zhang et al., 2015). Also mutations in P-body elements, like XRN4, DCP1, DCP2 and VCS, enhance the level of (RDR6-dependent) transgene silencing by PTGS (De Alba et al., 2015; Gazzani et al., 2004; Gy et al., 2007; Hayashi et al., 2012; Thran et al., 2012; Vogel et al., 2011; Yu et al., 2015; Zhang et al., 2015). In addition, mutations in several P-body elements induce a PTGS response against host RNA transcripts and can even turn plants lethal (De Alba et al., 2015; Gregory et al., 2008). These studies altogether indicate that a dysfunctional RQC leads to an enhanced PTGS response, due to aberrant RNAs from P-bodies now becoming available and increasing the amount of substrate for RDR6. This interaction also works the opposite way: Tobacco Mosaic virus (TMV) was shown to induce RNA decay pathways to impair the antiviral PTGS response (Conti et al., 2017). So, the RQC pathway and the RNAi pathway compete for the same aberrant RNA as substrate. Interestingly, an enhanced RNAi response can also protect against viral infections and indeed a XRN4 mutant is more resistant against the RNA viruses Cucumber mosaic virus (CMV) and Turnip mosaic virus (TuMV) (Gy et al., 2007; Vogel et al., 2011).

Whereas compromising RQC enhances the resistance against RNA viruses, this has not been tested for DNA viruses yet, like geminiviruses, and in specific Tomato Yellow Leaf Curl Virus (TYLCV). Recently, Ty-1, a resistance gene against TYLCV, has been cloned and shown to code for a RDR of the RDRY class. Further studies demonstrated that Ty-1 amplifies the RNAi response to TYLCV but does not protect against RNA viruses (Butterbach et al., 2014; Verlaan et al., 2013). Whether RDRs of the RDR $p$ class reside in P-bodies and whether they also compete with RQC pathways, just as RDR6, remains unclear.

Here we have investigated whether impairment of (cytoplasmic) RQC elements also enhances the antiviral RNAi response to DNA viruses. To test this hypothesis, P-body elements were silenced in Nicotiana benthamiana ( $N$. benthamiana) and 
the effect on susceptibility to TYLCV was monitored. While the (PTGS) silencing of a (control) GFP transgene was enhanced, no effects on TYLCV titers were observed and suggested that P-bodies do not seem to play a (major) role in antiviral defence against TYLCV.

\section{Results}

\section{Silencing of P-body elements}

Prior to challenging $N$. benthamiana compromised in the cytoplasmic RQC-system with TYLCV the efficacy of VIGS constructs to silence RQC elements from P-bodies was tested. To this end, $N$. benthamiana seedlings were infiltrated with TRV VIGS constructs containing part of the sequences of DCP2, DCP5 or VCS. As controls, an empty TRV VIGS vector ( $\mathrm{pTV}: 00$ ) and a TRV vector containing part of the gene phytoene desaturase (PDS), to visually monitor silencing in time, were used. Once photo-bleaching was observed in plants infiltrated with TRV-PDS, which was around $13 \mathrm{dpi}$, all plants infiltrated with other TRV constructs showed some stunting relative to untreated plants, indicating a successful TRV infection. Whereas TRV-DCP2 and TRV-DCP5 infiltrated plants showed a phenotype similar to those infiltrated with pTV:00, plants infiltrated with TRV-VCS were more severely stunted 35 dpi (Fig. 4.1).

To verify the knockdown of P-body elements i.e, confirm the silencing of DCP2, DCP5 and VCS, RNA was extracted from systemic leaves 35 days post TRV inoculation, and analysed by qRT-PCR to determine the relative expression levels of DCP2, DCP5 and VCS. The expression was normalised to the expression of the housekeeping gene Elongation Factor $1 \alpha(\mathrm{EF} 1 \alpha)$ and the fold difference in gene expression was calculated according to the DeltaDeltaCt method as described by Livak and Schmittgen (2001) (Fig. 4.2). The results revealed increased delta $\mathrm{Ct}$ values in the silenced plants, which indicate lower gene expression levels. The calculated fold differences showed that the expression of the three genes were lowered by approximately $80 \%$ in the silenced plants compared to the control (pTV:00 infiltrated) plants. 


\section{Silencing P-bodies enhances GFP transgene silencing}

Having confirmed the silencing of cytoplasmic RQC elements from P-bodies, the experiment was repeated and this time plants, at the stage when TRV-PDS silenced plants exhibited chlorophyll bleaching, were superimposed with a second agroinfiltration of a binary GFP construct. Since dysfunctional P-bodies have been reported to lead to an enhanced PTGS response, monitoring the silencing of the GFP transgene would confirm the experimental setup. Four days post GFP infiltration, leaf patches were harvested to quantify the relative fluorescence units. The GFP fluorescence was significantly lower in DCP2, DCP5 and VCS silenced plants compared to pTV:00 treated plants (Fig. 4.3), and indicated that the RNAi response targeting the GFP transgene expression was indeed enhanced upon silencing of P-body elements.
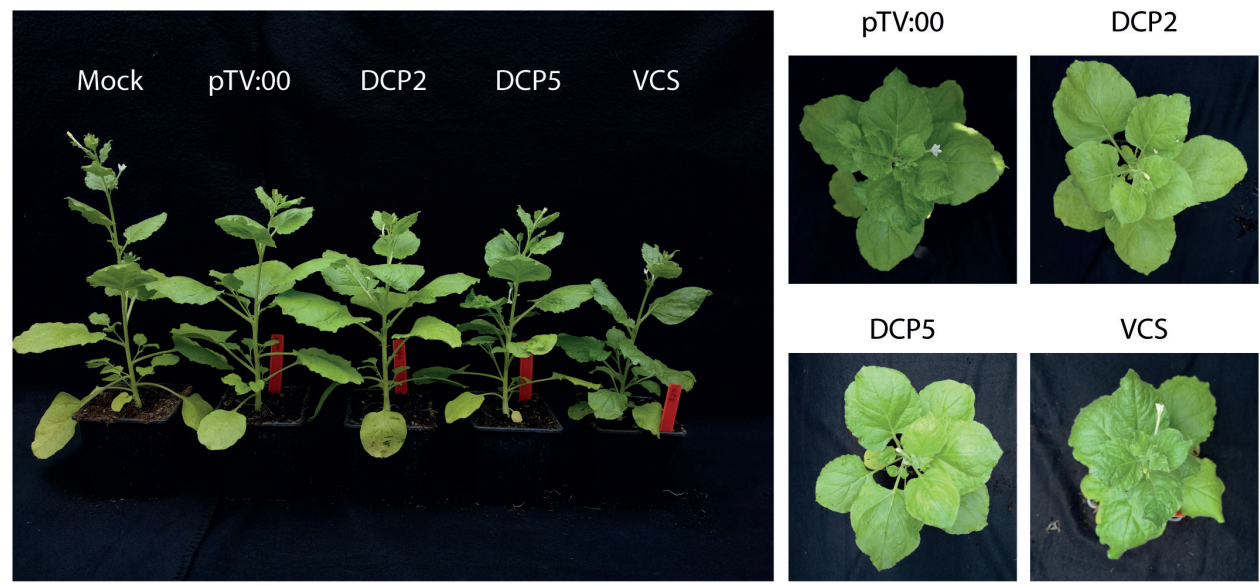

PDS

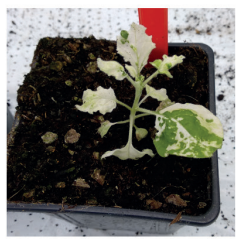

Figure 4.1: Phenotype of $\boldsymbol{N}$. benthamiana plants silenced on P-body elements.

Plants were infected with TRV containing sequences of RQC-elements DCP2, DCP5, VCS or PDS as a positive (visual) control. Pictures were taken 35 days post TRV infiltration. Left panel shows side views of (from left to right) a mock infected plant, pTV:00 infected plant, DCP2 silenced plant, DCP5 silenced plant and VCS silenced plant. The right panel shows a top view from the same plants and a PDS silenced plant. 


\section{Silencing P-bodies does not enhance TYLCV resistance}

Having confirmed that silencing of P-body elements, to compromise the cytoplasmic RQC, as expected led to enhanced (PTGS) silencing of a GFP transgene, next the effect of P-body silencing on the RNAi response targeting DNA viruses was tested. In analogy to previous experiments, $N$. benthamiana plants were first silenced for DCP2, DCP5 or VCS and 13 days post TRV infection now challenged with the DNA virus TYLCV. Symptoms of TYLCV were monitored over time and revealed no difference between plants silenced for different P-body elements relative to the negative control (Fig. 4.4A, symptoms 21 days post TYLCV infection). Samples of

Relative gene expression upon knockdown treatment

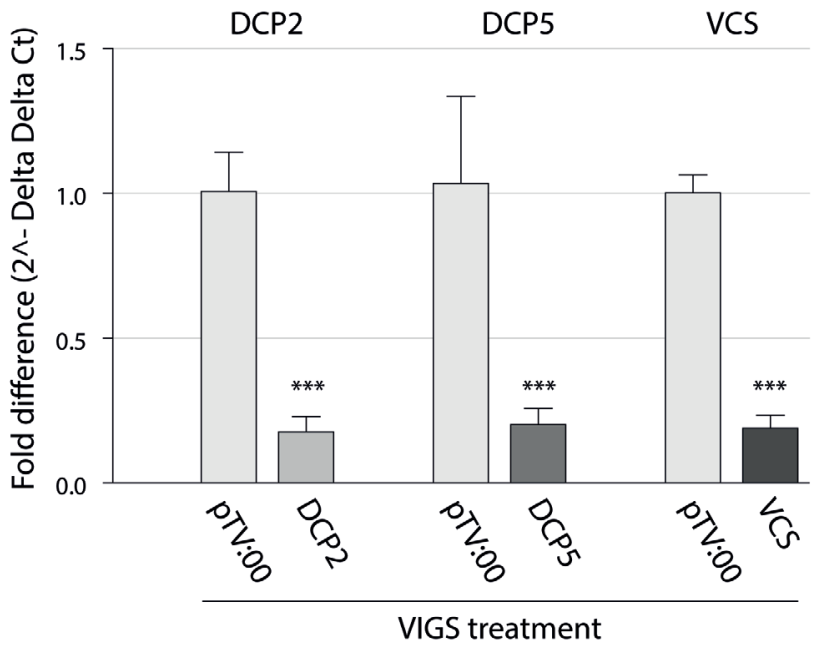

\begin{tabular}{|c|ccc|}
\hline Knockdown & Delta Delta CT & Fold difference (+/-SD) & Sig (2-tailed) \\
\hline DCP2 & 2,56 & $0.17(0.13-0.23)$ & 0,00 \\
DCP5 & 2,36 & $0.20(0.15-0.26)$ & 0,00 \\
VCS & 2,44 & $0.18(0.15-0.24)$ & 0,00
\end{tabular}

Figure 4.2: Relative gene expression of DCP2, DCP5 and VCS in VIGS-silenced plants compared to pTV:00 treated plants.

Thirty five days post TRV infection, samples were collected and RNA was isolated to determine gene expression levels via qRT-PCR. The levels of gene expression were calculated relative to the expression of the housekeeping gene EF1 $\alpha$. The graph shows the calculated relative fold difference via the $2^{\wedge}$-DeltaDeltaCt method, normalized to the unsilenced group (pTV:00, set to 1 ). The mean with standard deviation is depicted. $(* * *=$ P-value $<0.001$, based on the DeltaCt values). The table presents the calculated DeltaDeltaCt values and the fold differences. 
systemically infected leaves were taken 21 and 35 days post TYLCV infection. First, RNA was isolated and the silencing of the RQC elements was confirmed by qRT-PCR (data not shown). Subsequently, DNA was isolated to determine TYLCV viral titers, relative to the amount of genomic DNA, using GPCR. The results showed that the viral titers in samples subjected to different treatments were similar compared to the control group pTV:00 (Fig. 4.4B), and indicated that compromising the cytoplasmic RQC does not increase the RNAi defence response against TYLCV.

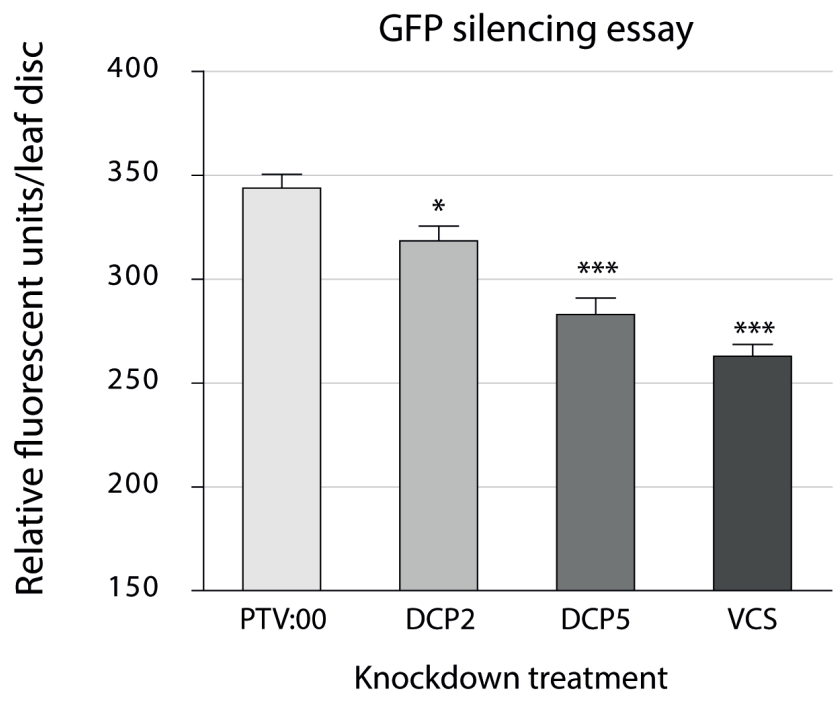

Figure 4.3: GFP expression 4 days post infiltration of a GFP transgene in $\mathbf{N}$. benthamiana plants unsilenced or silenced for DCP2, DCP5 or VCS.

Nicotiana benthamiana plants were silenced for DCP2, DCP5 or VCS expression via virus induced gene silencing using a TRV vector. Approximately 14 days post TRV infection, GFP was transiently expressed. Four days post GFP expression, leaf discs were taken and the relative fluorescence was measured with a plate reader. Mean GFP expression with standard error of the mean is depicted. Asterisks indicate significant differences between the silenced plants compared to unsilenced ( $\mathrm{pTV}: 00$ treated) plants according to one-way analysis of variance $(*=\mathrm{P}$-value $<0.05 ; * * *=\mathrm{P}$-value $<0.001)$. 
A. 21days post TYLCV infection

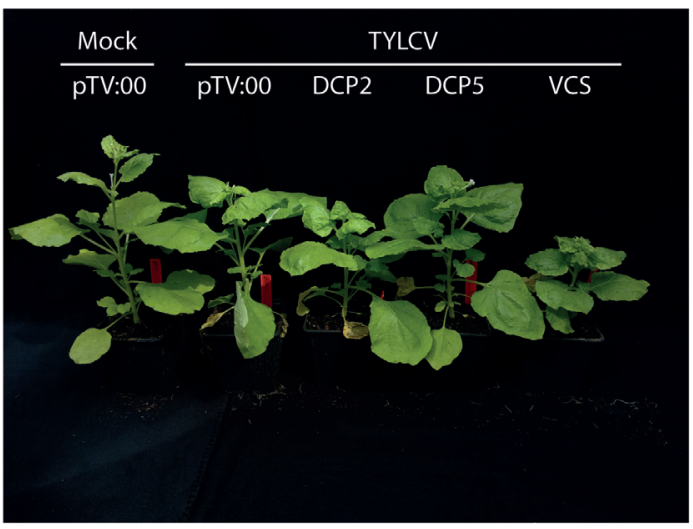

pTV:00

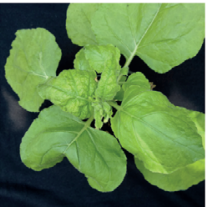

DCP5

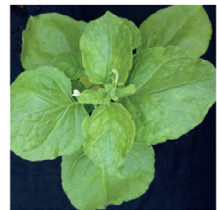

DCP2

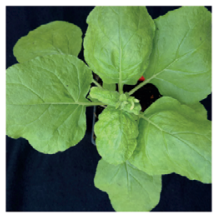

VCS

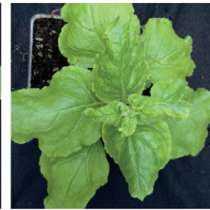

B.

Relative TYLCV titer, upon knockdown of P-Body elements

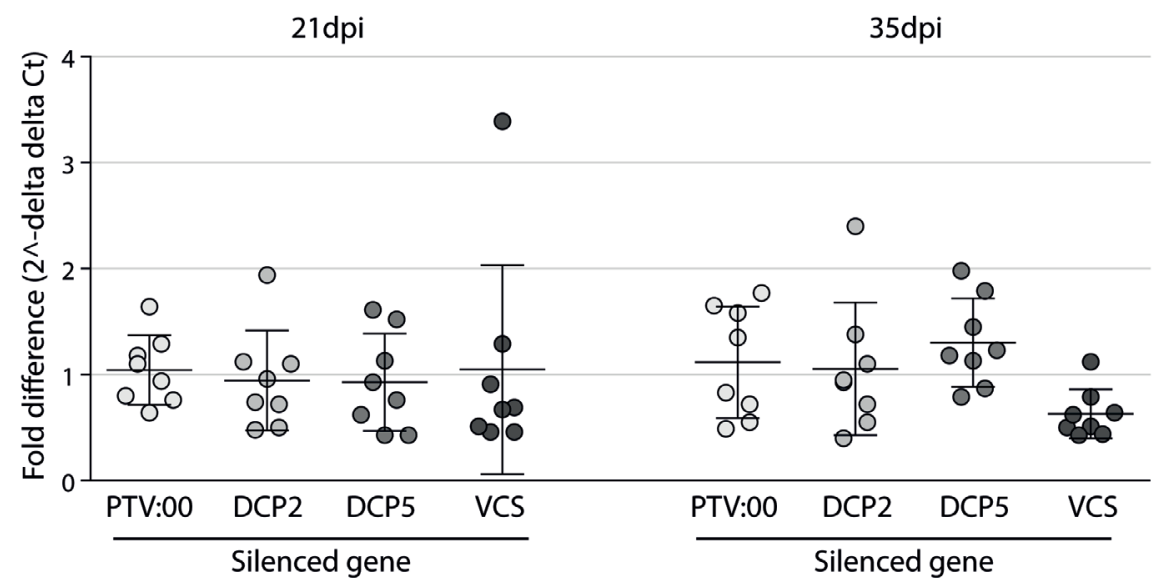

Figure 4.4: Symptoms of TYLCV infection and relative viral titers in unsilenced, DCP2, DCP5 and VCS silenced plants 21 and $\mathbf{3 5}$ days post infection.

Nicotiana benthamiana plants were infected with TRV constructs for VIGS silencing of P-body elements. Thirtheen days post TRV infection, the plants were agro-infected with an infectious clone of TYLCV. A. Symptoms of TYLCV 21 days post TYLCV infection. From left to right (side views), mock infected control previously infected with pTV:00, followed by TYLCV infected plants previously infected with pTV:00, pTV:DCP2, pTV:DCP5 and pTV:VCS respectively. Right hand side shows top views of the same TYLCV infected plants. B. At two different timepoints post TYLCV infection, systemic leaves were harvested. Total DNA was isolated and TYLCV titers were measured via qPCR. The viral titer was normalized to the presence of genomic DNA (25s rRNA) and the fold difference was calculated according to the $2^{\wedge}$-DeltaDeltaCt method relative to unsilenced (pTV:00) TYLCV infected plants (set to 1). Every point represents one plant. Lines represent means and standard deviation of biological replicates. No-significant difference was found according to one-way analysis of variance comparing the silenced plants to unsilenced (pTV:00 treated) plants. 


\section{Discussion}

Disruption of P-body elements, containing cytoplasmic RNA decay elements, has earlier been shown to increase the amount of aberrant RNAs as substrate for RDR6 amplification in neighbouring cytoplasmic siRNA bodies and leading to an enhanced PTGS response to RNA viruses and transgenes (De Alba et al., 2015; Gy et al., 2007; Kumakura et al., 2009; Thran et al., 2012). Whether this also leads to an increased RNAi response to DNA viruses has not been shown. This is interesting in light of recent findings that the Ty-1 resistance gene, which encodes a RDR of the $\gamma$-class, enhances the RNAi response against geminiviruses (Butterbach et al., 2014). The model plant Arabidopsis thaliana codes for three RDRs of the $\gamma$-class, however nothing is known about a potential role in antiviral RNAi as well as their cellular distribution. If RDRp proteins localize to siRNA-bodies, silencing of P-body elements could enhance their substrate supply as well. In contrary to the positive control using a GFP transgene, silencing of P-body elements to compromise the cytoplasmic RNA quality control did not increase the RNAi response to TYLCV. This was observed consistently during repeated experiments and where respectively DCP2, DCP5 and VCS were silenced.

Geminiviruses are prone to antiviral RNAi, both PTGS and TGS, but the contribution of both pathways in the anti-geminiviral defence is not completely clear. In general it is thought that TGS plays a more important role, since knock out of TGS elements enhance the virus susceptibility, but the knockout of DCL4 or RDR6 do not (Jackel et al., 2016; Raja et al., 2008). Interestingly, also the knock out of RDR2 does not enhance the susceptibility to geminiviruses and no drastic change is observed in antiviral siRNAs produced against the bipartite Cabbage Leaf Curl Virus (CaLCuV) in RDR1,2,6 triple knockout plants compared to wild type plants (Aregger et al., 2012; Raja et al., 2008). Therefore it remains unresolved which RDRs are important in mounting a strong RNAi response during a geminivirus infection and where they are localized. Considering the competition between P- and siRNA bodies for aberrant RNA substrate, silencing of P-body elements would lead to an enhanced RNAi response against TYLCV if RDRs resident in cytoplasmic siRNA bodies are involved in this (De Alba et al., 2015; Gy et al., 2007; Kumakura et al., 2009; Thran et al., 2012). Our studies indicated the contrary and supported the idea that most likely RDRs 
resident outside of cytoplasmic siRNA bodies play a role in enhancing the antiviral RNAi against geminiviruses. Altogether, this implies that $T y-1$, an RDR member of the gamma class and exerting resistance against TYLCV, is likely not localized to P-bodies but exerts its amplifying RNAi activity elsewhere (Butterbach et al., 2014; Verlaan et al., 2013).

It is generally assumed that PTGS (mainly) takes place in cytoplasmic siRNA bodies that localize nearby P-bodies, while several enzymes involved in TGS, such as DCL3 and AGO4, are found in the nucleus where also geminiviruses replicate (De Alba et al., 2015; Kumakura et al., 2009; Pontes et al., 2006). It is therefore reasonable that RDRs involved in the antiviral TGS response are localized in the nucleus and this confinement prevents a (rapid) substrate exchange with the cytoplasmic P-bodies. However, it is interesting to note that several RQC elements involved in $3^{\prime}$ to $5^{\prime}$ and $5^{\prime}$ to $3^{\prime}$ degradation are localized nuclear (Hématy et al., 2016; Li and Wang, 2018; Liu and Chen, 2016). Although speculative, it could be that silencing of these elements might change levels of aberrant nuclear RNA that affects the nuclear TGS response in a similar fashion as observed during the competition for aberrant RNAs between the cytoplasmic P-bodies and siRNA bodies.

It is reported that $N$. benthamiana, the model plant used in our study, is deficient in RDR1, and encodes a RDR6 and RDR2 (Nakasugi et al., 2013; Pérez-Cañamás et al., 2017; Wassenegger and Krczal, 2006; Yang et al., 2004), but nothing is reported about RDRs from the RDRp class, which are suggested to enhance the RNAi response against geminiviruses (Butterbach et al., 2014). A blast search on the predicted cDNA database of $N$. benthamiana with the Ty-1 coding sequence does show two sequences coding for a RDR $\gamma$, including a typical RDR $\gamma$ catalytic domain (DFDGD). In an online available transcriptome database of RNA isolated from leaves of $N$. benthamiana, several contigs completely match with the predicted cDNA (Divi et al., 2014). This shows that these RDRs of the $\gamma$-class encoded by $N$. benthamiana are expressed. However, whether these genes are functional to mount a RNAi response to geminiviruses is not known.

In this study, the knock down of P-body components did result in an increased GFP silencing, showing the PTGS response was enhanced. Some recent studies 
have suggested a role for PTGS in the defence response against geminiviruses. A knockdown of RDR6 led to hyper susceptibility against Tomato Yellow Leaf Curl China Virus (TYLCCV) (Li et al., 2014; Li, Wang, et al., 2017). In a recent study by Ye et al. (2015), a new P-body component was identified, Asymmetric leaves 2 (AS2), that is induced by the geminivirus Cabbage leaf curl virus (CaLCuV) BV1 protein and functions as an endogenous suppressor of PTGS. AS2 was observed to interact with DCP2 and enhance decapping. While AS2 overexpressing lines showed increased sensitivity to the bipartite CaLCuV and Indian cassava mosic virus (ICMV), AS2 KO plants exhibited an increased resistance to CaLCuV and altogether support an antiviral role of PTGS against geminiviruses. However, these findings are in contrast to observations earlier made by Raja et al. (2008), indicating no effect of knocking out PTGS components RDR6 and DCL4 on antiviral RNAi against geminiviruses, and from this study in which a knock down of P-body elements did not lead to an increased antiviral PTGS response to TYLCV. One aspect that can cause these different observations is the use of either knock out or knock down plants. During the RDR6 knockdown experiments described by Li et al. (2014, 2017), a transgenic $N$. benthamiana plant expressing dsRNA targeting RDR6 was used. It cannot be excluded that this knock down resulted in off-targets effects, affecting also other RDRs involved in different pathways (Schwach et al., 2005). It is also important to notice the use of other geminiviruses in these studies. In the study of Ye et al. (2015), bipartite geminiviruses were used and nothing was reported about the impact on monopartite geminiviruses. The conferred resistance by Ty-1 was found to be slightly less effective against bipartite than to monopartite geminiviruses (Prasanna et al., 2015). Whether this is caused by the fact that these geminiviruses code for other RSS proteins, inhibiting either PTGS or TGS more effectively (Rishishwar and Dasgupta, 2019) and leading to a difference in disease pathology, remains to be investigated.

The interplay between P-bodies, siRNA bodies and viral defence is not always the same. Some viruses interfere with P-bodies to support their replication (Xu et al., 2020). Decreasing RNA decapping led for example to increased susceptibility to Tomato spotted wilt virus (TSWV), instead of increased resistance, since TSWV uses RNA stored in P-bodies to snatch 5'-cap structures (Ma et al., 2019). In addition, turnip mosaic virus (TuMV) inhibits the RNA decay pathway. TuMV RNA 
accumulation is negatively affected by RNA decay, next to RNAi as defence response (Li and Wang, 2018). This shows that the interactions between these RNA granules and viruses is very complex.

Our findings implicate that no direct competition for RNA substrate takes place between P-bodies and siRNA (RDR6 containing) bodies that affect the RNAi response against TYLCV. In situ localization of Ty-1 and other RDRs of the $\gamma$ class will provide more insight in the role of these RDRs in the antiviral RNAi response against geminiviruses and the possible interplay between these RDRs and the nuclear RQC pathway.

\section{Experimental procedures}

\section{Plant material}

Throughout this study $N$. benthamiana plants were maintained under greenhouse conditions at $23{ }^{\circ} \mathrm{C}$ during the day and $21^{\circ} \mathrm{C}$ at night ( $16 \mathrm{~h}$ light $/ 8 \mathrm{~h}$ dark regime) and relative humidity of $60 \%$. The plants used for GFP silencing assays were kept in a climate chamber at $22{ }^{\circ} \mathrm{C}$, a relative humidity of $70 \%$ and a $16 \mathrm{~h}$ photoperiod.

\section{Constructs and viruses}

For the silencing of RNA quality control genes, Tobacco Rattle Virus (TRV) RNA2 constructs for DCP2, DCP5 and VCS were kindly provided by Peter Moffett (Department of Biology at the Université de Sherbrooke in Sherbrooke, Québec, Canada (unpublished)). As negative control, an empty TRV RNA2 construct (pTV:00) was used (Ratcliff et al., 2001). Constructs coding for TRV RNA 1 were used as described by Liu et al. (2002). All TRV constructs were transformed by electroporation to Agrobacterium tumefaciens (A. tumefaciens) GV3101 containing helper plasmid pSoup. For a transgene GFP silencing assay, pBin-GFP was transformed into $A$. tumefaciens LBA4404 (de Ronde et al., 2013). 
An agroinfectious clone of TYLCV Israelian strain isolated from Almeria, Spain, as described by Morilla et al. (2005) (GenBankAJ489258.1) was used for TYLCV infection. For infection, the agroinfiltration protocol as described under Agrobacterium Transient Transformation assay (ATTA) was followed with an $\mathrm{OD}_{600}$ of 0.5.

\section{Agrobacterium Transient Transformation assay (ATTA)}

ATTAs were performed following the protocol earlier described (Bucher et al. , 2003) with minor modifications as described in Chapter 2. In brief, A. tumefaciens was grown overnight at $28{ }^{\circ} \mathrm{C}$ in $3 \mathrm{ml}$ LB3 (10 gL-1 trypton, 5 gL-1 yeast, 4 gL-1 NaCl, $\left.1 \mathrm{gL}-1 \mathrm{KCl}, 3 \mathrm{gL}-1 \mathrm{MgSO}_{4} \cdot 2 \mathrm{H}_{2} \mathrm{O}\right)$ medium containing proper antibiotic selection pressure. From this culture, $600 \mu \mathrm{l}$ was transferred to $3 \mathrm{ml}$ induction medium (10.5 gL-1 $\mathrm{K}_{2} \mathrm{HPO}_{4}, 4.5 \mathrm{gL}-1 \mathrm{KH}_{2} \mathrm{PO}_{4}, 1 \mathrm{gL}-1\left(\mathrm{NH}_{4}\right) 2 \mathrm{SO}_{4}, 0.5 \mathrm{gL}-1$ Sodium citrate $2 \mathrm{H}_{2} \mathrm{O}, 1 \mathrm{mM}$ $\mathrm{MgSO}_{4} .7 \mathrm{H}_{2} \mathrm{O}, 0,2 \%(\mathrm{w} / \mathrm{v})$ glucose, 0,5\% (v/v) glycerol, $50 \mu \mathrm{M}$ acetosyringone, 10 $\mathrm{mM}$ MES ( $\mathrm{pH}$ 5.6)) and grown overnight. The next day bacteria were pelleted by centrifugation (15 min. 2670g) and resuspended in MS MES buffer (murashige and skoog medium (Duchefa biochemia) supplemented with $150 \mu \mathrm{M}$ acetosyringone, $10 \mathrm{mM}$ MES and $87 \mathrm{mM}$ sucrose). The $\mathrm{OD}_{600}$ was determined and adjusted to the desired $O D_{600}$. Plants were watered in excess 1 hour before infiltration and subsequently infiltrated by pressure inoculation with a needle-less syringe on the abaxial side of the leaf.

\section{Virus Induced Gene Silencing (VIGS)}

A co-ATTA was performed on 2-to-3-week-young $N$. benthamiana seedlings using A. tumefaciens carrying a binary construct of the TRV2 replicon containing (partial) sequences of the gene of interest (DCP2, DCP5, VCS, PDS or no insert) and $A$. tumefaciens carrying TRV1. Agrobacterium tumefaciens carrying TRV2 and TRV1 were mixed $1: 1$ to a final $O D_{600}$ of 0.5 per construct. Seedlings that were infiltrated with VIGS-TRV constructs were agroinfiltrated in their systemic leaves with binary GPF constructs or an agroinfectious clone of TYLCV, once VIGS-PDS control seedlings exhibited bleaching of chlorophyll. 


\section{GFP silencing assay}

Nicotiana benthamiana leaves were agroinfiltrated with pBin-GFP (ATTA) at a final $\mathrm{OD}_{600}$ of 0.5 . Fluorescence of GFP was quantified 4 days post infiltration (dpi) from 4 leaf discs/leaf ( $1 \mathrm{~cm}$ in diameter) from the infiltrated leaf area using a Fluorstar Optima (BMG Labtech, excitation filter $485 \mathrm{~nm}$, emission filter $520 \mathrm{~nm}$ ). Data was analysed using the software Microsoft Excel 2010 and IBM SPSS statistics 22.

\section{Nucleic acid isolation}

Systemically infected leaves were harvested 3 or 5 weeks post TYLCV infection and snap frozen in liquid nitrogen. The material was stored at $-80^{\circ} \mathrm{C}$ until further processing. For TYLCV titer determination, total DNA was isolated following the cetyltrimethyl ammonium bromide (CTAB) protocol (Doyle and Doyle, 1987) with slight modifications as described by Fulton et al. (1995). DNA concentration was measured by a Nanodrop ND-1000.

For gene expression analysis, RNA was extracted (from the same samples as used for DNA extraction) using Trizol following the manufacturers' protocol (Invitrogen). Total RNA concentration was determined by a Nanodrop ND-1000.

\section{Virus titration}

Virus titers of TYLCV were determined by qPCR using 25s rRNA as internal control. Quantified PCR was performed in a reaction mixture containing $1 x$ SyberSelect (Thermo Fischer Scientific), 300 nM TYLCV specific forward primer (5'-ttcgtctagatattccctatatgaggaggta-3'), 300 nM TYLCV specific reverse primer (5'- gggaagcccattcaaattaaagg- $\left.3^{\prime}\right)$ and $10 \mathrm{ng}$ DNA. For amplification of $25 \mathrm{~s}$ rRNA 25s-forward primer (5'-ataaccgcatcaggtctcca-3') and 25s-reverse primer (5'-ccgaagttacggatccattt- $3^{\prime}$ ) were used. The qPCR was performed in a Bio-Rad CFX 384 using the following PCR conditions: 2 min $95^{\circ} \mathrm{C}$, followed by 40 cycles of $15 \sec 95^{\circ} \mathrm{C}$ and $1 \mathrm{~min} 60^{\circ} \mathrm{C}$, followed by a melting curve with $0.5^{\circ} \mathrm{C}$ steps from 
$60{ }^{\circ} \mathrm{C}$ to $95^{\circ} \mathrm{C}$ to determine PCR specificity. Viral titers were calculated using the DeltaDeltaCt method as described by Livak and Schmittgen (2001). Values were normalized relative to the internal control $25 \mathrm{~S}$ rRNA, and calibrated to levels of the control plants, which were set as 1 .

\section{Gene expression analysis}

For gene expression analysis $1 \mu \mathrm{g}$ of purified total RNA was treated with TURBO DNAse according to the manufacturers' recommendations (Invitrogen). Reverse transcription was performed on 400 ng total RNA using random hexamers (Roche) and M-MLV reverse transcriptase according to the manufacturers' protocol (Promega). Quantified PCR was performed on first strand CDNA to determine the expression levels of DCP2, DCP5 and VCS relatively to the housekeeping gene EF1 $\alpha$. The qPCR was performed in a total volume of $10 \mu$ containing $1 x$ SyberSelect (Thermo Fischer Scientific), $300 \mathrm{nM}$ forward primer, $300 \mathrm{nM}$ reverse primer and $2 \mu \mathrm{l} 5$ times diluted cDNA. The following primers were used for PCR amplification: for DCP2 primers DCP2-F (5'- acctagactggatagaccttca-3') and DCP2-R (5' -ggcttgttccattgaatctcag-3'), for DCP5 primers DCP5-F (5' - gaccacctacaacatcggca-3') and DCP5-R (5'-aggcaaagtagcagcaggtaa-3'), for VCS primers VCS-F (5'-tcctcatctggtccttcagt-3') and VCS-R (5'-ggtcacagacatggctttct-3'), for EF1 $\alpha$ primers EF1 $\alpha-F\left(5^{\prime}\right.$-agctttacctcccaagtcatc-3') and EF1 $\alpha-R$ (5'-agaacgcctgtcaatcttgg-3'). The qPCR was performed in a Bio-Rad CFX384 using the following PCR conditions: 2 min $95^{\circ} \mathrm{C}$, followed by 40 cycles of $15 \mathrm{sec} 95^{\circ} \mathrm{C}$ and $1 \mathrm{~min} 60^{\circ} \mathrm{C}$, followed by a melting curve with $0.5^{\circ} \mathrm{C}$ steps from $60^{\circ} \mathrm{C}$ to $95^{\circ} \mathrm{C}$ to determine PCR specificity. Gene expression levels were calculated using the DeltaDeltaCt method as described by Livak and Schmittgen (2001). Values were normalized relative to the internal control EF1 $\alpha$, and calibrated to levels of the control plants, which were set as 1 . In addition, the statistical significance was determined using the delta CT-values in IBD SPSS Statistics 22. 


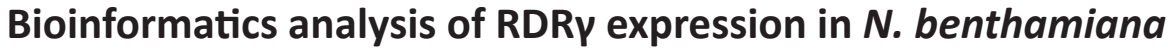

An online available transcriptome database of RNA isolated from leaves of six weeks old N. benthamiana plants was used (Divi et al., 2013). The published assembled and annotated contigs were screened for RDRs. Those selected contigs were compared with the sequences of RDR1 to RDR6 and Ty-1 by performing a multiple sequence alignment using Clustal Omega (default settings)(Madeira et al., 2019). If a contig covered the catalytic domain, it was checked whether it codes for the typical RDR $\alpha$ (DLDGD) or RDRy (DFDGD) catalytic domain. In addition, the generated phylogenetic tree was checked for grouping with either RDR1, RDR2 and RDR6 or RDR3, RDR4 and RDR5. Next, a BLAST search was performed with the Ty-1 coding sequence on the $N$. benthamiana predicted cDNA database (solgenomics.net, $N$. benthamiana genome v1.0.1 predicted cDNA). The BLAST hits were aligned with the selected contigs from the transcriptome database (DFDGD domain or clustering with RDR3/RDR4/RDR5) and with the contigs which were already annotated as RDR3, RDR4 or RDR5.

\section{Acknowledgements}

The authors would like to thank Prof. Eduardo Rodríguez Bejarano (Universidad de Málaga, Malaga, Spain) for providing the infectious TYLCV (Almeria isolate) clone. We thank Peter Moffett (Department of Biology at the Université de Sherbrooke in Sherbrooke, Québec, Canada) for providing the TRV RNA2 constructs for silencing of DCP2, DCP5 and VCS. This research was financially supported by NWO-CNPq within the Joint Research Project Biobased Economy (729.004.011). 


\section{Chapter 5}

Ty-1 encoded RNA-dependent RNA polymerase from the gamma class, conferring resistance to geminiviruses, localizes to nuclear bodies involved in RNAi response and sumoylation

C.M. Voorburg*, M.J. Mazur*, M. Ravensbergen, M. D'aniello, D. Lohuis, R. Kormelink

\footnotetext{
* These authors contributed equally to this work
} 


\section{Summary}

The Ty-1 resistance gene encodes an RNA-dependent RNA polymerase of the uncharacterized $\gamma$-class and enhances the RNA interference response against geminiviruses. However, the involvement of Ty-1 in either the cytoplasmic post-transcriptional gene silencing (PTGS), or the nuclear transcriptional gene silencing (TGS) response remained to be elucidated. The Ty-1 gene was cloned in fusion with GFP and its localization was studied in Nicotiana benthamiana leaves. In addition, interactions with co-localizing proteins were examined in bimolecular fluorescence complementation assays. Ty-1 was shown to localize in a small number of nuclear protein bodies, while in the additional presence of the geminivirus tomato yellow leaf curl virus (TYLCV) the number of Ty-1 nuclear bodies increased. The Ty-1 protein neighboured photobodies, which changed to a (partial) co-localization with Cajal bodies during TYLCV infection. Ty-1 co-localized and interacted with photobody component COP1 and with components of the sumoylation machinery, namely SIZ1, SCE1, SUMO1 and SUMO3. TYLCV infection influenced the interplay with the sumoylation machinery. In addition, TYLCV upregulated and activated the sumoylation machinery. The nuclear localization of Ty-1 is a strong indication that the resistance mechanism functions via the TGS response, while the additional data point towards an involvement of sumoylation in the Ty-1-mediated response. 


\section{Introduction}

Tomato Yellow Leaf Curl Virus (TYLCV) belongs to the most harmful plant viruses worldwide and can cause up to $100 \%$ yield losses in valuable crops like tomato. The virus is widely distributed but especially occurs in tropical and subtropical areas due to its insect vector, the whitefly Bemisia tabaci, being endemic to these regions (Moriones and Navas-Castillo, 2000). TYLCV is the representative of the (monopartite) begomoviruses within the family Geminiviridae. Virions are composed of capsid protein and a single stranded (ss) circular DNA genome of $2.6 \mathrm{~kb}$. The genome contains six open reading frames (ORFs), two of which are oriented in viral ( $\mathrm{V}$, or clockwise) sense, and four in complementary ( $\mathrm{C}$, anticlockwise) sense (Zerbini et al., 2017). The capsid protein is encoded by V1, while V2 encodes the viral movement protein. C1 to C4 encode replication-associated protein (Rep), transcriptional activator protein, replication enhancer protein and protein $\mathrm{C} 4$, respectively, and they all play a role in viral DNA genome replication. This process occurs in the nucleus and depends on C1/Rep to induce the expression of host genes to initiate cell proliferation and viral DNA replication (Arguello-Astorga et al., 2004; Nagar et al., 1995). Viral genome replication occurs by homologous recombination and rolling-circle replication (Jeske et al., 2001).

During geminivirus infection, next to modulating gene expression, Rep modulates the sumoylation of host genes via interactions with the SUMO E2 CONJUGATING ENZYME (SCE1) (Sanchez-Duran et al., 2011). Sumoylation is a post translational modification (PTM), in which the small peptide SUMO is attached to a protein by the catalytic action of SCE1. The model plant Arabidopsis thaliana encodes eight SUMO peptides, named SUMO1 to SUMO8, from which SUMO1, 2, 3 and 5 are known to be expressed (Kurepa et al., 2003). SUMO1 and 2 are 89\% identical and are the main substrate for conjugation. Sumoylation can be promoted by the enzyme SUMO E3 ligase SAP and Miz 1 (SIZ1) (Miura et al., 2007). Sumoylation always takes place on lysine residues $(\mathrm{K})$ and in most cases within the sumoylation consensus motif $\psi \mathrm{KxD} / \mathrm{E}$, where $\psi$ is a hydrophobic and $\mathrm{x}$ any amino acid residue (Hendriks et al., 2015; Matic et al., 2010; Rodriguez et al., 2001; Sampson et al., 2001). Other proteins can interact with sumoylated proteins via a short hydrophobic peptide stretch, called a SUMO interaction motif (SIM), and thereby may affect the 
stability/functionality and/or localization of sumoylated proteins (Hecker et al., 2006; Kerscher, 2007). Sumoylation is known to also play a role in the regulation of plant immunity. A nice example is the modulation of the NB-LRR resistance gene SNC1 (Gou et al., 2017). Furthermore, mutants of the sumoylation pathway show changed defence responses, as recently reviewed (Verma et al., 2018). While Rep modulates sumoylation of host genes, interestingly, the interaction of Rep with SCE1 also appears essential for begomovirus replication (Sanchez-Duran et al., 2011) and indicates an important role for sumoylation in the establishment of a viral infection.

During viral infection, geminiviruses are prone to RNA interference (RNAi, also referred to as RNA silencing). RNAi is a highly conserved mechanism responsible for e.g. genome maintenance and regulation of gene expression, but this mechanism can also turn antiviral upon the synthesis of viral dsRNA (Ding and Voinnet, 2007). During geminivirus infection, overlapping ( $\mathrm{V}$ - and C-gene) transcripts or folding structures in mRNAs that give rise to dsRNA molecules, are considered to be the trigger for antiviral RNAi (Ramesh et al., 2017). Double-stranded RNA molecules are recognized and subsequently cleaved into small interfering RNA (siRNA) molecules of 21-24 nucleotides (nt) by Dicer-Like proteins (DCL). One strand of the siRNA is uploaded into a RNA-Induced Silencing Complex (RISC) that contains a core Argonaute (AGO) protein with slicer activity (Hammond, 2005). Arabidopsis thaliana plants contain four DCL proteins, of which DCL2 and DCL4 are involved in the biogenesis of 21-22 nt siRNAs (Parent et al., 2015). These activate AGO1containing RISC to survey and target RNA molecules with sequence complementarity to the siRNA strand for degradation or translational arrest, a process called post transcriptional gene silencing (PTGS) (Mallory and Vaucheret, 2010). This process plays a major role in the defence against RNA viruses but is also activated upon geminivirus infection and targets viral mRNA (Aregger et al., 2012; Kuria et al., 2017; Piedra-Aguilera et al., 2019; Yang, Wang, et al., 2011). Biogenesis of $24 \mathrm{nt}$ siRNAs involves cleavage of dsRNA by DCL3 and their strands preferentially upload into an AGO4-containing RISC, to guide RNA-dependent DNA methylation (RdDM). The activated RISC will target methyltransferases to complementary DNA sequences and methylate cytosine residues, causing transcriptional gene silencing (TGS) of the DNA sequence (Mallory and Vaucheret, 2010). TGS is a major player in antiviral defence against geminiviruses, since both DCL3 and AGO4 knock out (KO) plants are 
hypersusceptible to geminiviruses (Raja et al., 2008).

Plants generally mount a strong RNAi response due to amplification of the RNAi signal, absent from mammals, by host-encoded RNA-dependent RNA polymerases (RDRs) (Donaire et al., 2008; Wang et al., 2010). These polymerases convert aberrant RNA molecules, resulting from the slicer activity of AGO, into dsRNA, which then will become processed by DCL and generate a pool of secondary siRNAs. The Arabidopsis thaliana genome codes for six RDRs, named RDR1 to -6. The function of RDR1, -2 and -6 , belonging to the $\alpha$-class, is well studied, while the function of RDR3, -4 and -5 , belonging to the $\psi$-class, is unknown (Willmann et al., 2011). RDR1 and -6 are involved in the amplification of 21-22 nt siRNAs, i.e. the PTGS response, and silencing of these genes turn plants highly susceptible to RNA viruses (GarciaRuiz et al., 2010; Leibman et al., 2011; Qi et al., 2009; Qu, 2010; Rakhshandehroo et al., 2009; Wang et al., 2010). DCL4, AGO1 and RDR6 present the major players of the PTGS response and localize to the cytoplasm. DCL4 distributes diffusely while AGO1 and RDR6 localize to spatially linked processing- and siRNA bodies, respectively (Mäkinen et al., 2017; Montavon et al., 2017). From the $\alpha$-class, RDR2 is involved in the (canonical) TGS response and converts ssRNA, produced by RNA polymerase IV in the nucleus, into dsRNA molecules. Processing of the latter by DCL3 leads to the production of 24 nt siRNAs to guide RdDM (Li et al., 2006; Qi et al., 2006). The main players of TGS, i.e. RDR2, DCL3 and AGO4, localize in nuclear Cajal bodies (CBs) (Li et al., 2006; Pontes et al., 2006). DCL3 and Coilin double KO plants, which fail to form proper CBs, show decreased levels of methylation compared to DCL3 single KO plants, indicating the requirement of CBs for TGS (Li et al., 2008; Love et al., 2017). In line with this, Coilin-silenced plants become more susceptible against the geminivirus Tomato golden mosaic virus (TGMV) (Shaw et al., 2014). While several studies have demonstrated a role of RDR1 in the biogenesis of 21-22 nt viral siRNAs and antiviral activity towards RNA viruses, it is less understood than RDR6 and RDR2. RDR1 is induced upon viral infection or salicylic acid (SA) treatment and has also been implicated in the production of (virus-activated) endogenous siRNAs to confer broad spectrum antiviral activity complementary to the resistance conferred by virus-specific siRNAs (Cao et al., 2014; Xie et al., 2001; Yu et al., 2003). Furthermore, RDR1 has been linked to a recently identified, non-canonical $21 \mathrm{nt}-$ directed RdDM pathway (Pontier et al., 2012; Stroud et al., 2013; N., Wang et al., 
2014), but so far, no studies have reported on the cellular localization of RDR1. Not much is known on RDRs from the $\gamma$-class either.

Recently, Ty-1, a major single dominant resistance gene deployed to combat TYLCV, has been cloned from Solanum chilense (S. chilense) and revealed to code for an RDR from the $\gamma$-class (Verlaan et al., 2013). Ty-1 bearing plants infected with TYLCV, do not show disease symptoms nor a hypersensitive response and only exhibit low virus replication levels. The RDR has been demonstrated to enhance the antiviral RNAi response (Butterbach et al., 2014), as observed from increased antiviral siRNA production levels and methylation of the geminivirus DNA genome. To further investigate the involvement of Ty-1 in the PTGS and/or TGS response, an in situ localization study was performed. Results showed that Ty-1 localized juxtapose to nuclear photobodies and this localization changed to CBs upon viral infection, and that Ty-1 interplayed with the sumoylation machinery.

\section{Results}

\section{Ty-1 localizes to nuclear bodies and upon viral infection changes in spatial distribution}

To indicate whether Ty-1, as a member of the RDR $\gamma$-class, is involved either in the cytoplasmic PTGS or nuclear TGS response, Ty-1 was in situ localized. To this end, Ty-1 was cloned into a transient expression vector fused to an N-terminal GFP tag under control of a $35 \mathrm{~S}$ promoter. To stabilize its expression the GFP-Ty-1 construct was agroinfiltrated together with a construct coding for the RNA silencing suppressor protein P19 for expression in Nicotiana benthamiana (N. benthamiana) leaves and this was done in all subsequent localization experiments. To first verify expression of Ty-1, proteins were extracted $3 \mathrm{dpi}$ and analysed on a western blot using anti-GFP antibodies. However, no protein band of $\sim 136 \mathrm{kDa}$ (estimated size for GFP-Ty-1) was observed. Even upon enrichment by immunoprecipitation no band indicative for GFP-Ty-1 or for a smaller degradation product, was discerned on western blot (data not shown). When the enriched samples were analysed by Mass Spectrometry, peptides specific for GFP-Ty-1 were identified covering the protein from the $\mathrm{N}$ to 
the $\mathrm{C}$ terminal side (Fig. 5.1A), indicating that GFP-Ty-1 was fully expressed although at relatively low levels.

In a next step, GFP-Ty-1 was expressed in N. benthamiana and leaves were collected at three dpi for in situ localization of Ty-1 by confocal microscopy. Although a weak GFP signal was observed in both cytoplasm and nucleus, the signal was predominantly localized to nuclear bodies (NBs) (Fig. 5.1B). This observation was consistently made during repeated experiments. While the amount of nuclear GFP-Ty-1 bodies varied per cell, in general between 0 and 9 bodies were observed with a median of 4 to 5 bodies per cell (Fig. 5.1C).

To analyse whether a TYLCV infection changed the number and spatial distribution of the Ty-1 NBs, GFP tagged Ty-1 was co-infiltrated with an agro-infectious clone of TYLCV (Morilla et al., 2005). While Ty-1 was still observed in the nucleus in NBs, the number of NBs was clearly increased, ranging from 0 to 32 bodies per cell with a median of 11 (Fig. 5.1B,C).

To test whether the increase in number of Ty-1 bodies was due to viral replication or caused by one of the viral proteins only, plants were agroinfiltrated with GFP-Ty-1 in the presence of single 35S-driven constructs coding for one of the TYLCV proteins (V1, V2, C1 to C4) N-terminally tagged with mRFP. Prior to this experiment, the expression and localization of the viral proteins fused to RFP was verified by agroinfiltration of the single viral protein gene constructs. In agreement with previous observations, V1 and C3 were enriched in the nucleolus, C2 was present in the nucleoplasm only, $\mathrm{C} 1$ and $\mathrm{C} 4$ were divided over nucleoplasm and cytoplasm, whereas V2 was present in the cytoplasm of the cell (Sup. Fig. 5.1A) (Dong et al., 2003; Hak et al., 2015; Nagar et al., 1995; Teng et al., 2010; Wang et al., 2017; Van Wezel et al., 2001). Upon co-expression of GFP-Ty-1 and the RFP tagged viral proteins, no increase in number of Ty-1 NBs was observed. To our surprise, in a few nuclei V1 condensed to a NB that co-localized with the Ty-1 protein body (Sup. Fig. 5.1B). Furthermore, no Ty-1 bodies were observed in the presence of $\mathrm{C} 3$ or C4 (Sup. Fig. 5.1B). Altogether these results indicated that the observed increase in number of nuclear Ty-1 bodies upon TYLCV infection was likely due to viral replication and not to the presence of single viral proteins. 
A.

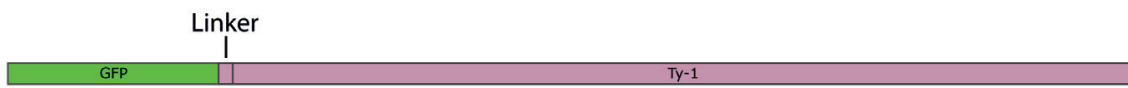

민

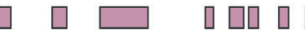

B.

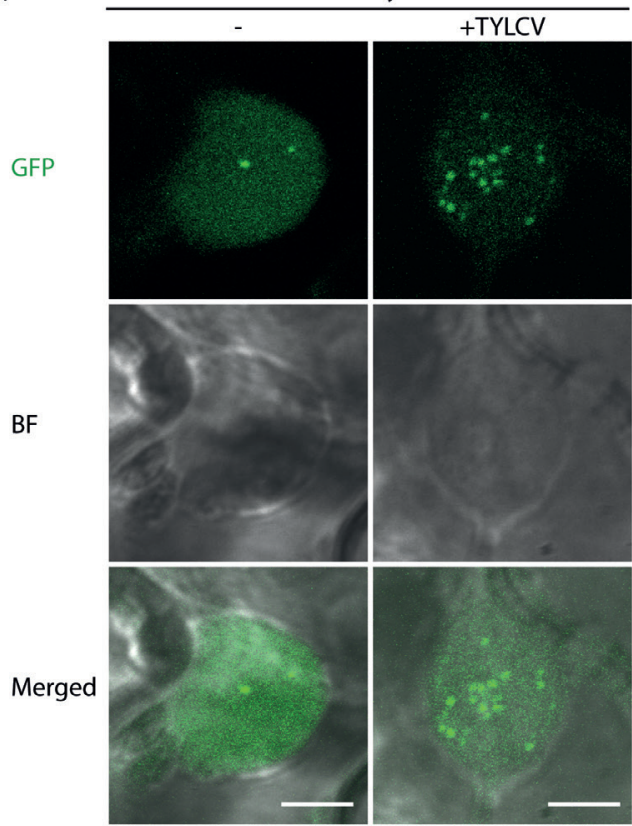

C.

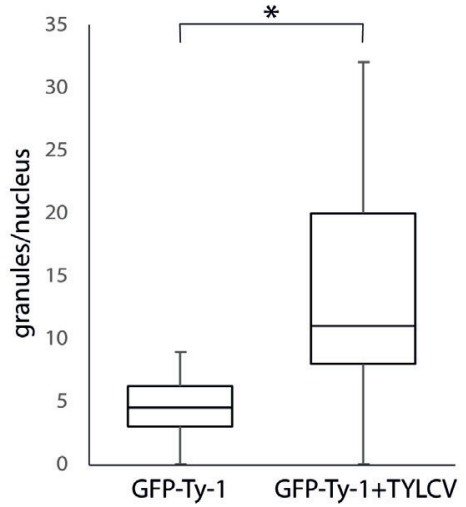

Figure 5.1: Ty-1 localizes to nuclear bodies and the localization changes upon viral infection.

A. GFP-Ty-1 was expressed via an ATTA in Nicotiana benthamiana leaves and harvested 3 dpi. The protein was pulled down using anti-GFP antibodies and the enriched fraction analysed using mass spectrometry. A schematic drawing of the protein and the identified peptides are depicted. B. The localization of GFP-Ty-1 visualized via confocal microscopy, either GFP-Ty-1 alone or co-infiltrated with the infectious clone of TYLCV. Fluorescence was detected $3 \mathrm{dpi}$. The scale bars represent $5 \mu \mathrm{m} \mathrm{C}$. A boxplot showing the quantification of the amount of Ty-1 nuclear bodies per nucleus in the absence or presence of the virus. Per sample at least 16 nuclei were counted. Asterisks indicate significant differences between GFP-Ty-1 alone or in the presence of TYLCV according to a Mann-Whitney $U$ test $(*=p<0.05)$.

\section{In non-infected plant cells, Ty-1 localizes to NBs juxtaposed to COP1 photobodies}

The localization of Ty-1 to NBs implicated a role in the nuclear TGS response, a process in which CBs are known to play a major role. To examine whether Ty-1 
localizes to CBs, GFP-Ty-1 was co-expressed with mRFP-tagged constructs coding for marker proteins for nucleolus and CBs (Fibrillarin 2 (Fib2) derived from both Arabidopsis thaliana and N.benthamiana (named AtFib2 and NbFib2 respectively)) and for $\mathrm{CB}$ only (U2B"). After marker proteins were verified for proper expression and localization (Sup. Fig. 5.2), they were co-expressed with GFP-Ty-1 (Fig. 5.2A). Ty-1 did not co-localize with CBs (Fib2 and U2B", Fig 5.2A, column 1, 2 and 3). To further identify the nature of Ty-1 NBs, GFP-Ty-1 was co-expressed with mRFP-tagged markers of other structures present in the nucleus, namely the nucleolus (RPL24B), dicing bodies (HYL1), and photobodies (CONSTITUTIVE PHOTOMORPHOGENIC 1 (COP1)). Ty-1 did not co-localize to nucleolus (RPL24B and Fib2, Fig. 5.2A, column 1, 2 and 4), nor dicing bodies (HYL1, Fig. 5.2A, column 5). Instead, Ty-1 was consistently found in granules juxtaposed to COP1-containing photobodies (Fig. 5.2A, column 6, Fig. 5.2B).

\section{During viral infection, Ty-1 co-localizes to CBs}

Considering that viral infection affected the number of nuclear Ty-1 bodies, we tested for a possible change in the nature of Ty-1 bodies when expressed in the presence of TYLCV. To this end, the localization of GFP-Ty-1 in TYLCV infected $N$. benthamiana leaf epidermal cells was studied via co-expression with the marker protein constructs. Interestingly, Ty-1 bodies did not localize juxtaposed to photobodies anymore, neither co-localized to nucleolus or dicing bodies (Fig. 5.3). Only in a few cases, Ty-1 still was discerned in bodies juxtaposed to COP1, similar to the situation in the absence of a viral infection (Fig. 5.2A). Instead, and interestingly, in the majority of cases Ty-1 (partly) co-localized to the CB marker AtFib2/NbFib2 (Fig. 5.3). While in some cells Ty-1 and Fib2 fully co-localized, mostly only part of Fib2 and Ty-1 bodies co-localized. These observations were consistently made with the CB markers AtFib2 and NbFib2, but not with U2B". While the latter CB marker normally is expressed well, in the presence of a TYLCV infection this protein was difficult to detect.

Next to the observed changes in Ty-1 bodies during viral infection, also the number of Fib2 bodies increased and their appearance became more diffused in 
A. GFP-Ty-1

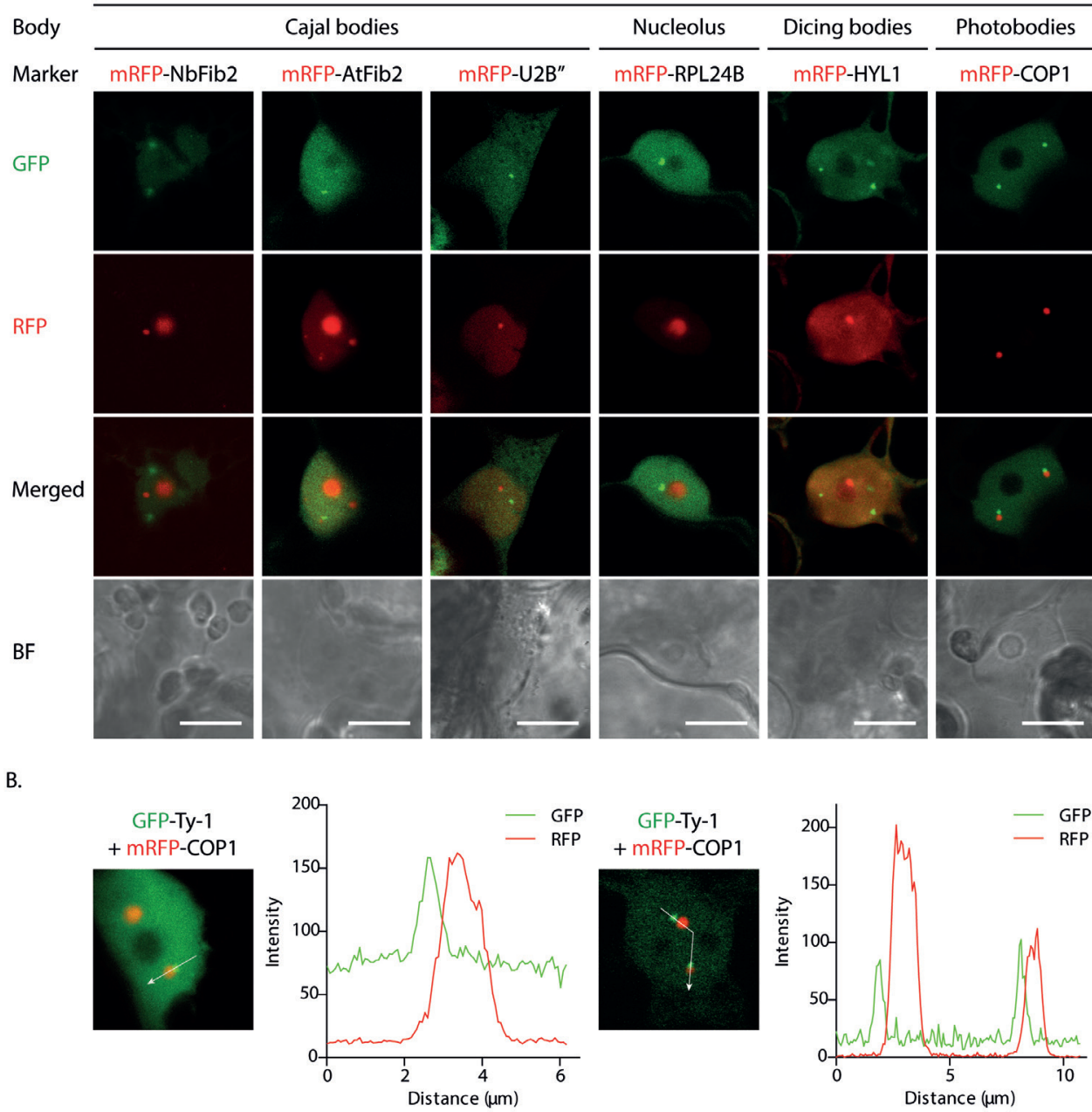

Figure 5.2: Ty-1 nuclear bodies localize adjacent to COP1 containing photobodies

A. GFP-Ty-1 and RFP tagged marker proteins for cajal bodies, nucleolus, dicing bodies and photobodies were co-expressed using agroinfiltration and the localization analysed $3 \mathrm{dpi}$ using the confocal microscope. The scale bars represent $10 \mu \mathrm{m}$. B. Fluorescent intensity plot of GFP-Ty-1 and RFP-COP1 co-expressing cells. The intensity of GFP and RFP along the arrow is depicted.

the presence of TYLCV infection. To test whether the same shift would also take place in the presence of TYLCV infection but without Ty-1 and whether this was specific for Fib2 or would also occur for other CB markers, $C B$ markers Fib2 and 


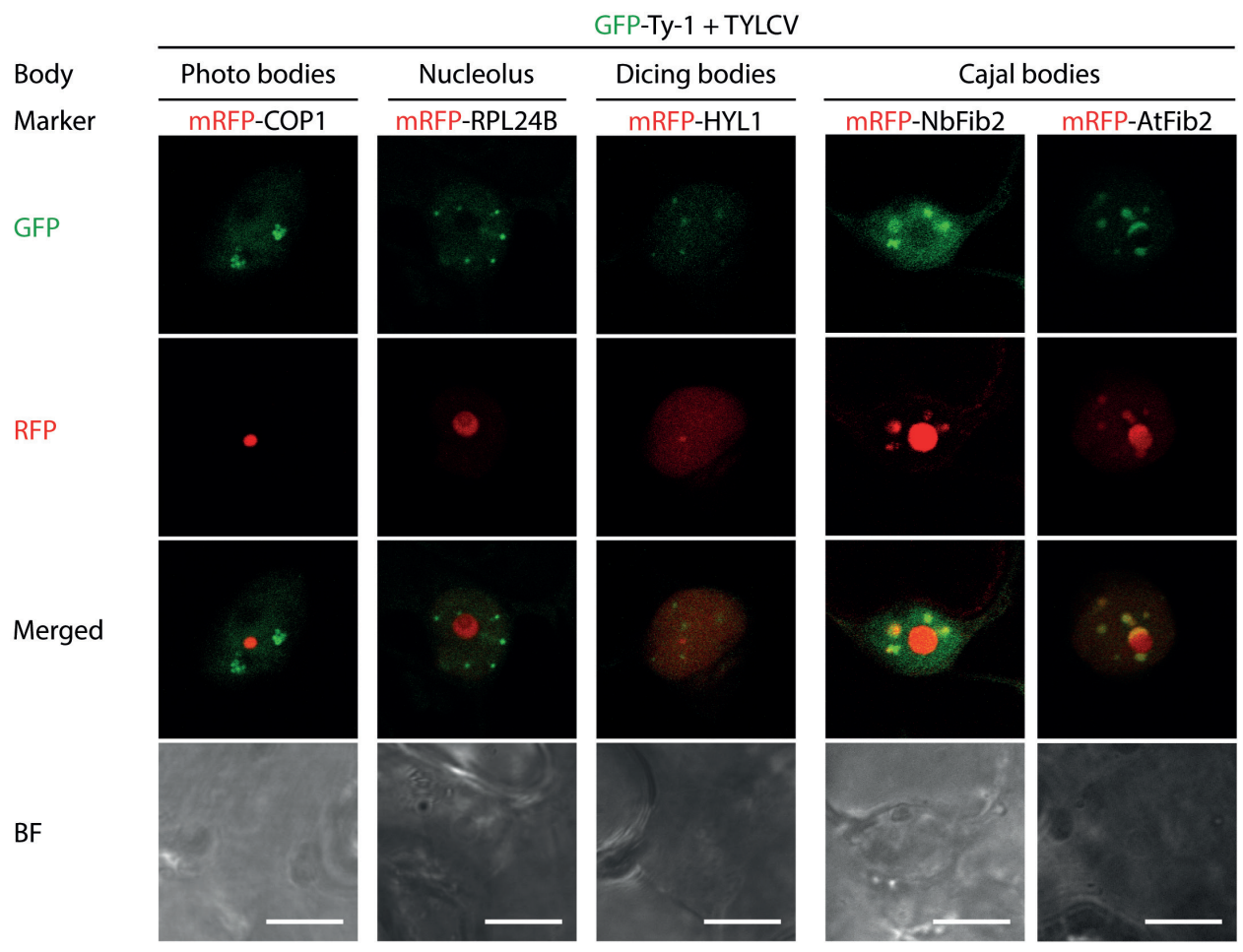

Figure 5.3: Ty-1 co-localizes with Fib2 during TYLCV infection

RFP tagged marker proteins, GFP tagged Ty-1 and an infectious clone of TYLCV were transiently expressed in $N$. benthamiana cells using agroinfiltration. The samples were analysed $3 \mathrm{dpi}$ using the confocal microscope. The localization of GFP-Ty-1 and RFP tagged marker proteins for photobodies (COP1), the nucleolus (RPL24B), dicing bodies (HYL1) and CBs (NbFib2 and AtFib2) are depicted in the presence of TYLCV. The scale bars represent $10 \mu \mathrm{m}$.

U2B" (N-terminally fused to either RFP or GFP) were co-expressed with and without TYLCV. Without TYLCV, GFP-U2B" co-localized with RFP-U2B", Nb-Fib2 and At-Fib2 (Sup. Fig. 5.3). However, in contrast to earlier observations, GFP-U2B" was also found in the nucleolus. Upon viral infection, GFP-U2B" showed an increased number of NBs, while no bodies could be detected for RFP-U2B" due to low expression levels (Sup. Fig. 5.3). Fib2 also seemed to show an increase in number of NBs, which often co-localized to GFP-U2B" (Sup. Fig. 5.3), altogether indicating that upon TYLCV infection the number of $\mathrm{CBs}$ increased. 


\section{$T y-1$ interacts with the sumoylation machinery}

To examine whether Ty-1, when localized juxtaposed to COP1-photobodies, interacts with COP1, a Bimolecular Fluorescence Complementation (BiFC) study was performed. To this end, Ty-1 was N-terminally tagged with SCFPN (N-terminal half of the super Cyan fluorescent protein [sCFP]), while COP1 was N-terminally tagged with SCFPC (C-terminal half of SCFP). When both constructs were co-expressed in the absence of a viral infection, SCFP fluorescence was observed concentrated in nuclear foci (Fig. 5.4A). These foci fully co-localized with mRFP-COP1, but not with AtFib2, indicating that Ty-1 translocated upon interactions with COP1 to COP1containing photobodies (Fig. 5.4B). When both constructs were co-expressed in the presence of a viral infection the SCFP signal was decreased. This indicated a (partial) loss of interaction (Fig. 5.4A) and was in agreement with earlier observations that showed a relocalization of Ty-1 from neighbouring COP1 bodies to CBs during a viral infection (Fig. 5.3).

Since COP1-bodies are physically connected to NBs of the SUMO machinery via COP1-SIZ1 interactions, and these structures also contain other components like SCE1, and SUMO1/2 peptides (Mazur et al., 2019), the localization of Ty-1 relative to those components was investigated. To this end, first, SIZ1, SCE1, SUMO1 and SUMO3 were expressed as $\mathrm{N}$-terminal fusion proteins with mRFP. In agreement with previous observations (Mazur et al., 2019), mRFP-SIZ1, mRFP-SCE1, mRFP-SUMO1 localized to nucleoplasm, while mRFP-SUMO3 was observed in the nucleoplasm and nucleolus (Fig. 5.5 and Sup. Fig 5.4). Interestingly, the presence of GFP-Ty-1 induced the formation of granular condensations of SCE1 and SUMO3, but not of SIZ1 nor SUMO1 (Fig. 5.5 and Sup. Fig. 5.4). The Ty-1-induced condensation bodies of SCE1 either fully overlapped or were located in close proximity to the Ty-1 NBs (Fig. 5.5). Moreover, in the presence of the virus, SIZ1 translocated into granules and, in the additional presence of Ty-1, localized around Ty-1 NBs (Fig. 5.5). TYLCV infection only, i.e. in the absence of Ty-1, also induced the formation of SUMO1, SUMO3 and SCE1 granules (Fig. 5.5 and Sup. Fig 5.4). However, and interestingly, the condensation of SCE1 was not observed when both Ty-1 and the virus were present and instead only a weak, diffused nucleoplasmic signal of SCE1 was observed (Fig. 5.5). 
A.

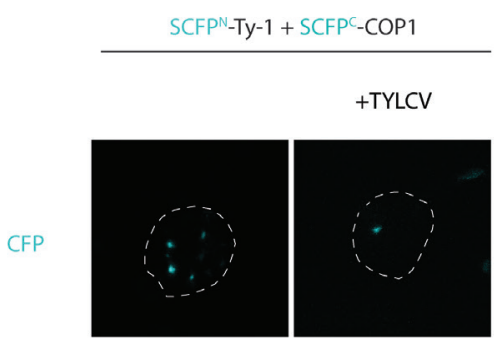

B.

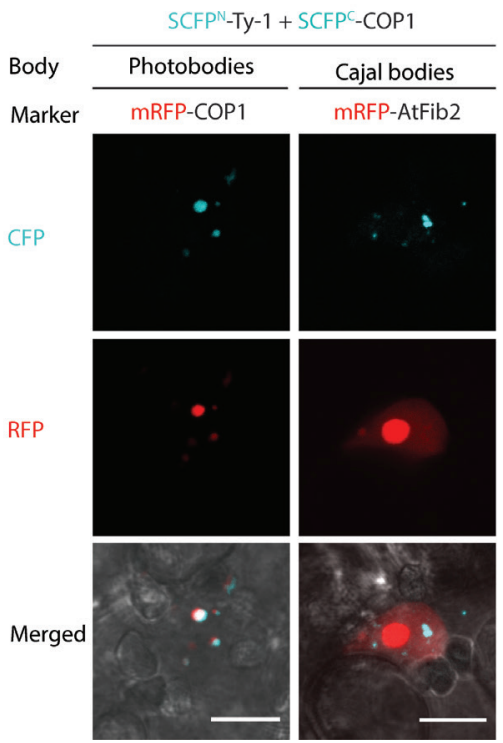

Figure 5.4: Ty-1 interacts with COP1 and this interaction changes during viral infection.

A. Ty-1 was expressed as fusion protein with the N-terminal half of SCFP (SCFPN), together with COP1 as fusion protein with the C-terminal half of SCFP (SCFPC) in N. benthamiana cells. In some samples additionally the infectious clone of TYLCV was infiltrated. Leaf cells were imaged $3 \mathrm{dpi}$ using the confocal microscope and the reconstituted CFP signal of the split SCFP proteins is depicted. The scale bars represent $10 \mu \mathrm{m}$. B. Ty-1 was expressed as fusion protein with the N-terminal half of SCFP (SCFPN), together with COP1 as fusion protein with the C-terminal half of SCFP (SCFPC) together with RFP tagged markers for photobodies (COP1) and CBs (AtFib2). Leaf cells were imaged 3 dpi using the confocal microscope and the signals for CFP, RFP and a merge with the bright field are depicted. The scale bars represent $10 \mu \mathrm{m}$.
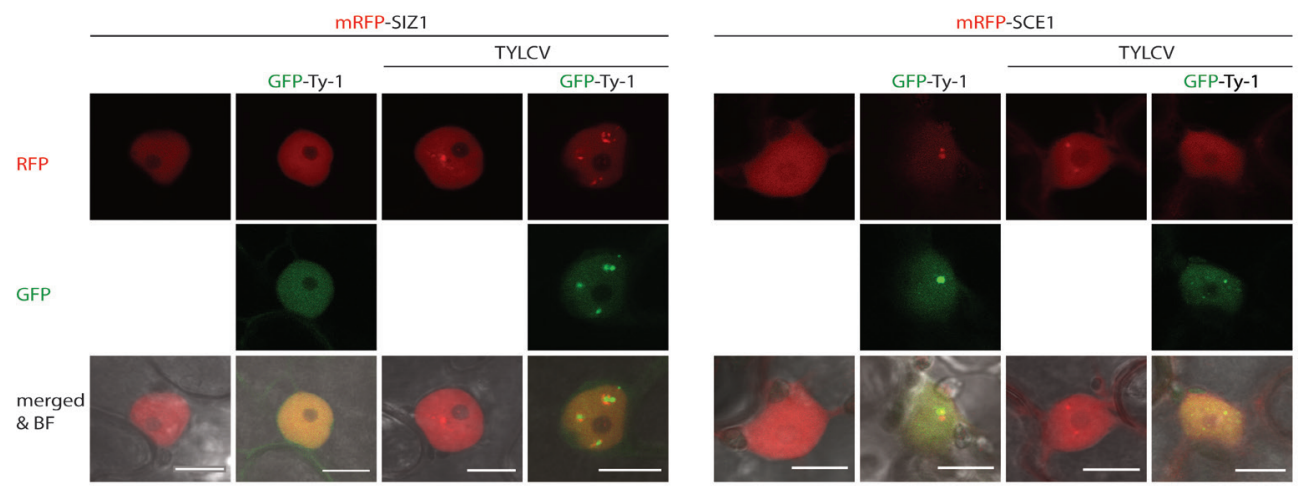

Figure 5.5: Ty-1 and TYLCV influence the formation of NBs of SIZ1 and SCE1.

SCE1 or SIZ1 fused to RFP was expressed in N. benthamiana leaves via ATTA, either in the presence of Ty-1 fused to GFP, or in the presence of TYLCV or both GFP tagged Ty-1 and TYLCV. The cells were imaged 3 dpi with a confocal microscope using RFP and GFP filters. The scale bars represent $10 \mu \mathrm{m}$. 
The change in spatial distribution of SCE1 and SUMO3 in the presence of Ty-1, indicated a Ty-1-driven re-localization to NBs in an interaction-specific manner. In order to test this hypothesis, BiFC assays were performed. To this end, Ty-1 was N-terminally fused to SCFPN, whereas SIZ1, SCE1, SUMO1 and SUMO3 were $\mathrm{N}$-terminally fused to SCFPC. For all the tested combinations of Ty-1 and SUMO machinery proteins, a distinct nuclear signal was detected (Fig. 5.6A and Sup. Fig. 5.5). Co-expression of SCFPN-Ty-1 and SCFPC-SIZ1 resulted in SCFP signal uniformly distributed in the nucleoplasm, but not in the nucleolus, while co-expression of SCFPN ${ }^{\mathrm{N}}$-Ty-1 and SCFPC-SCE1 resulted in a clear SCFP signal in protein bodies within the nucleus (Fig. 5.6A). In the presence of a TYLCV infection the signals from Ty-1SIZ1 and Ty-1-SCE1 complexes seemed to reduce (Fig. 5.6A). Although a SCFP signal was observed when SCFPN ${ }^{\mathrm{N}}$-Ty1 was co-expressed in the presence of SCFPC-SUMO1 and SCFPC-SUMO3, the intensity of the fluorescence was weak and only found in a few nuclei (Sup. Fig. 5.5). When BIFC assays were performed in the additional presence of nuclear marker proteins the Ty-1-SCE1 complexes co-localized to the nucleolus (RPL24B), CBs (AtFib2) and photobodies (COP1), but not to dicing bodies (HYL1) (Fig. 5.6B).

\section{Expression of host genes influenced by TYLCV and the presence of Ty-1}

To study whether Ty-1 or TYLCV also affected the transcriptional expression of the aformentioned enzymes of the SUMO machinery, the expression level was determined via a Reverse-Transcriptase quantitative PCR (RT-qPCR) in uninfected and TYLCV infected susceptible tomato Moneymaker (MM) and resistant Ty-1 bearing tomato plants (introgression line). It was observed that the expression of several homologs of SCE1 and SIZ1 is increased 30-70\% upon TYLCV infection in MM plant, but not in Ty-1 plants (Fig. 5.7). Actually, none of the tested genes was upregulated in TYLCV-infected Ty-1 plants compared to uninfected Ty-1 plants, most likely due to a very low replication level of the virus which stays under the threshold of defence response induction. As a next step, the expression of the marker genes which localization correlated with Ty-1 were checked (Fig. 5.7). Fib2, the marker for CBs is induced upon TYLCV infection in MM plants, while Fib2 expression in Ty-1 
A.

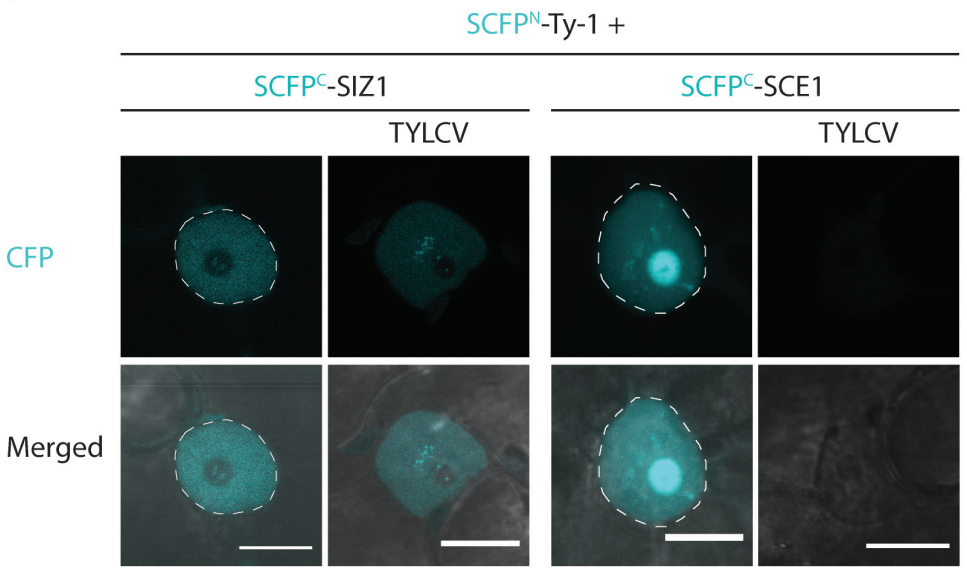

B.

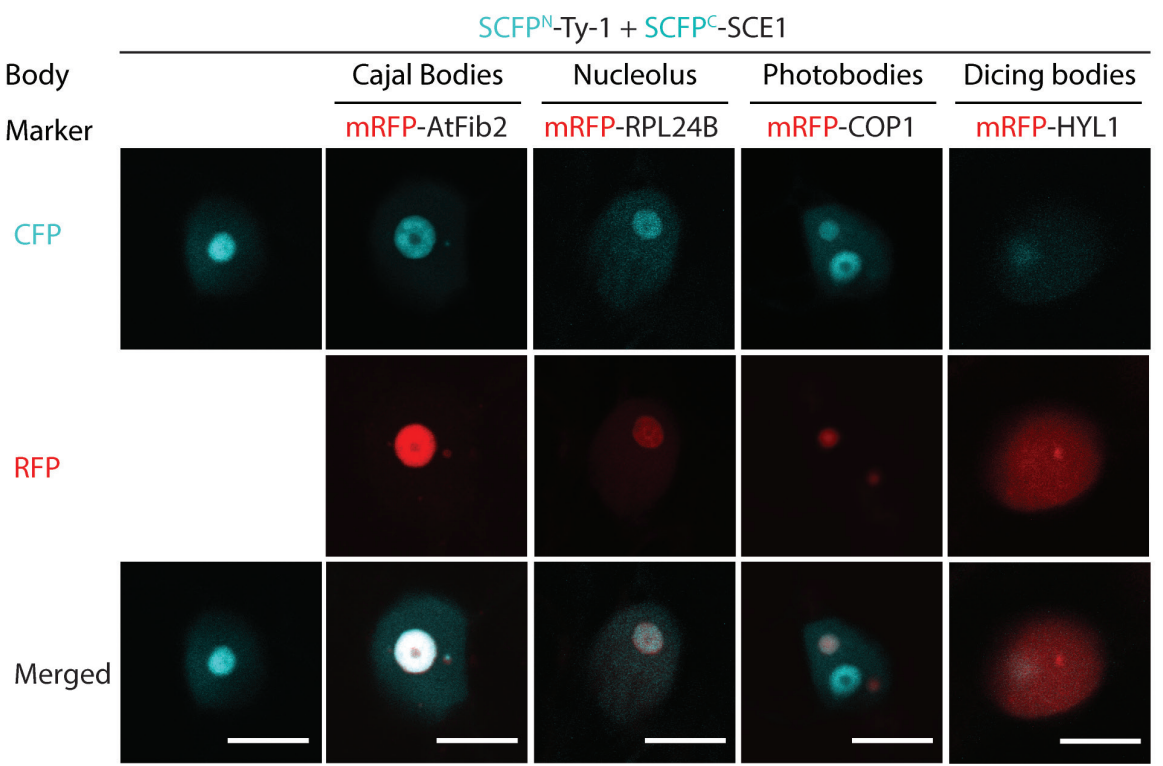

Figure 5.6: Ty-1 interacts with components of the sumoylation machinery, which changes upon TYLCV infection.

A. Ty-1 was expressed as fusion protein with the N-terminal half of SCFP (SCFPN), together with either SIZ1 or SCE1 as fusion protein with the C-terminal half of SCFP (SCFPC) in N. benthamiana cells via ATTA and the reconstituted CFP signal imaged 3 dpi using the confocal microscope. In some samples, TYLCV was co-expressed via co-infiltration of an infectious clone of TYLCV. The scale bars represent $10 \mu \mathrm{m}$. B. Ty-1 was expressed as fusion protein with the N-terminal half of SCFP (SCFPN), together with SCE1 fused to the C-terminal half of SCFP (SCFPC) and RFP tagged markers for either CBs (AtFib2), nucleolus (RPL24B), photobodies (COP1) or dicing bodies (HYL1) in N. benthamiana cells via ATTA. Three dpi, CFP and RFP signals were imaged using the confocal microscope. The scale bars represent $10 \mu \mathrm{m}$. 
plants is lower than in MM plants. Interestingly and in contrast to Fib2, one of the COP1 homologs was twice as high expressed in Ty-1 plants compared to MM plants, strengthening the idea of a role for COP1 in Ty-1 function or regulation.

Gene expression in susceptible (MM) and resistant (Ty-1) tomato plants upon TYLCV infection

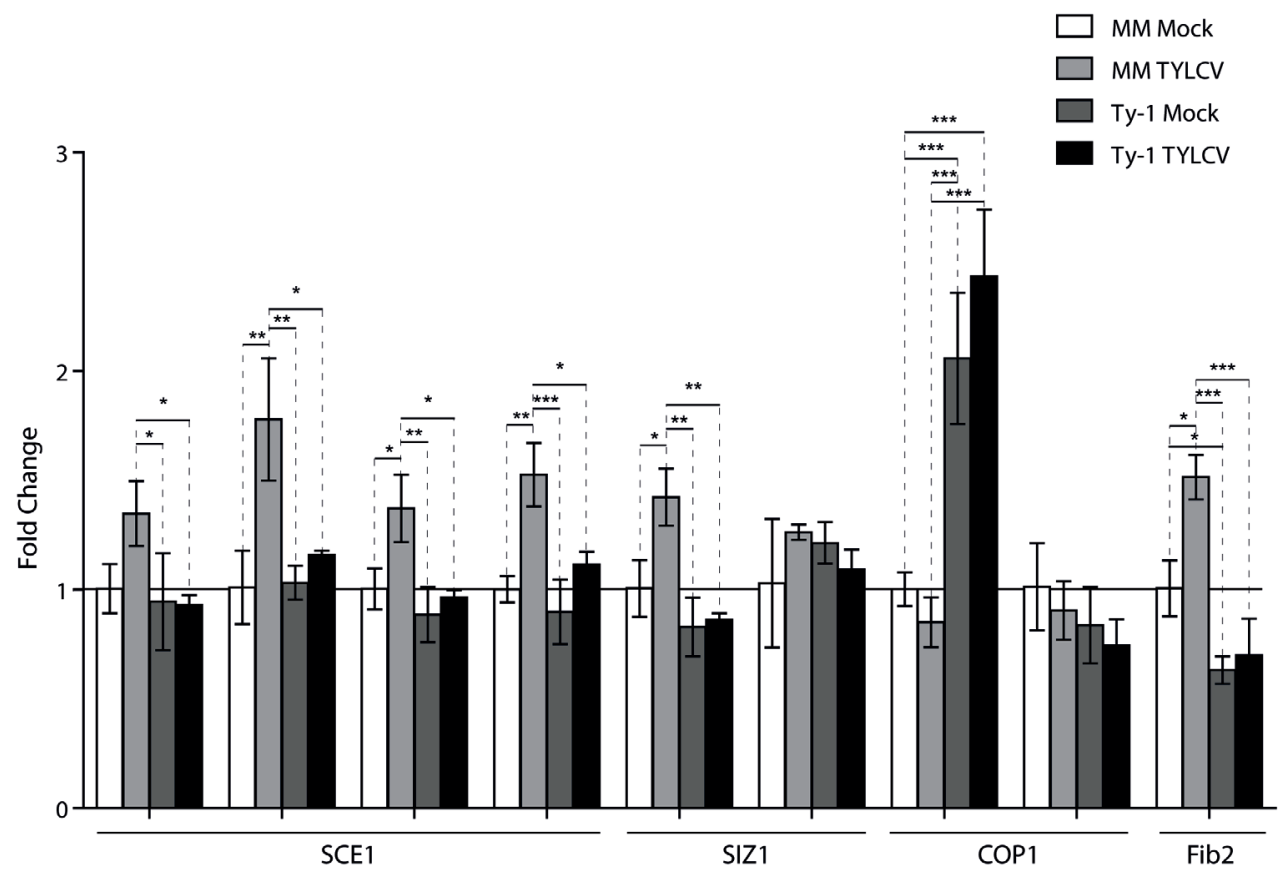

Figure 5.7: TYLCV infection induces the expression of the SUMO machinery and Ty-1 induces COP1 expression.

RNA was isolated from systemic leaves from uninfected and infected susceptible MoneyMaker (MM) and resistant Ty-1 plants (introgression line), all groups in triplo. Homologous genes of the sumoylation machinery, COP1 and Fib2 in tomato were determined via sequence homology with genes present in Arabidopsis thaliana, and via homologs annotated in the PANTHER database. The expression of these genes was measured via a RT-qPCR, calibrated to housekeeping genes and the fold change was calculated compared to susceptible uninfected (Mock treated) MM plants (set to 1 ) via the $2^{\wedge}$-DeltaDeltaCt method. Average expression levels with the standard deviation of the different homologous genes of SCE1 (Solyc04g078620, Solyc12g088680, Solyc02g093110 and Solyc03g044260), SIZ1 (Solyc06g010000 and Solyc11g069160), COP1 (Solyc11g011980 and Solyc12g005950) and Fib2 (Solyc03g025270) are depicted. Asteriks represent significant difference between the groups according to one way-analysis of variance on the DeltaCt levels, with a post hoc Tukey's Multiple Comparison Test $\left({ }^{*} p<0.05, * * p<0.01,{ }^{* * *} p<0.001\right)$. 


\section{Discussion}

Ty-1 presents an atypical dominant resistance gene that encodes a RDR of the $\gamma$-class and enhances the antiviral RNAi response against TYLCV. In this study, the Ty-1 encoded RDRy protein has been in situ localized in healthy $N$. benthamiana and shown to concentrate into nuclear protein bodies that are spatially linked to COP1 containing photobodies. In the presence of TYLCV replication, but not during a transient co-expression with viral proteins, a clear redistribution of Ty-1 was observed. Not only the number of Ty-1 protein bodies increased, but Ty-1 also relocalized from neighbouring COP1 photobodies to CBs. In addition, Ty-1 was observed to interact with SUMO-1,-3, SIZ1 and SCE1, important players of the sumoylation machinery, and these interactions changed during TYLCV infection. These results altogether indicated a role of sumoylation in Ty-1 governed antiviral TGS.

The nuclear localization of Ty-1 most resembles RDR2 which also has been shown to localize to CBs (Li et al., 2006; Love et al., 2017; Pontes et al., 2006). There, RDR2 together with DCL3 and AGO4 plays an essential role in the production of 24 nt siRNAs from heterochromatic regions, endogenous transposons and repeat elements to direct DNA methylation and/or histone modifications at their target sites, ultimately leading to TGS (Kawakatsu and Ecker, 2019; Li et al., 2006; Li et al., 2008; Pontes and Pikaard, 2008; Qi et al., 2006). Also geminiviruses are inhibited via TGS, since geminivirus DNA becomes methylated during an infection and hypermethylation correlates with a recovery phenotype (Ceniceros-Ojeda et al., 2016; Coursey et al., 2018). In addition, V2 encoded by the geminivirus Cotton leaf curl Multan virus (CLCUMuV) inhibits the TGS response by binding to AGO4 (Wang et al., 2019). However, whether exactly the same pathway is involved as for inhibiting e.g. transposable elements is not completely resolved. Arabidopsis KO plants of the main players DCL3 and AGO4 are hypersusceptible to the geminiviruses CaLCuV and/or BCTV (Jackel et al., 2016; Raja et al., 2008). The same is found for KO plants of polymerase IV, important upstream in the TGS pathway, and for KO plants of several downstream players, like DDM1 (chromatin remodelling enzyme), cytosine methyltransferases, histone methyltransferase and methyl cycle enzymes (Raja et al., 2008). Interestingly, RDR2 KO plants do not show this phenotype and 
therefore provided first evidence for the involvement of other antiviral RDRs in TGS against geminiviruses (Raja et al., 2008). The re-distribution of Ty-1 towards CBs and the earlier observation that the rate of cytosine methylation is increased in viral DNA collected from tomato plants bearing the $T y-1$ resistance gene, relative to susceptible plants (Butterbach et al., 2014) implies that Ty-1 contributes to antigeminiviral TGS.

The pathway leading to the initiation of a TGS response against geminiviruses remains somewhat elusive. Polymerase IV and V are not needed for the initiation of methylation of geminiviral genomes but rather to maintain and reinforce TGS (Jackel et al., 2016). Recently, some alternative non-canonical TGS pathways have been identified that connect the methylation of DNA with components of the PTGS pathway (Bond and Baulcombe, 2015; McCue et al., 2015; Nuthikattu et al., 2013; Panda and Slotkin, 2013; Pontier et al., 2012; Wu et al., 2012). One of those, denoted as RDR6 directed DNA methylation pathway, is involved in the initiation of cytosine methylation of transposable elements (Nuthikattu et al., 2013; Panda and Slotkin, 2013). In this pathway RDR6 converts RNA polymerase II transcripts into dsRNA and these are next processed by either DCL2/4 into 21-22 nt siRNAs. One strand of the siRNA loads into AGO4 or 6 instead of AGO1, to guide the methylation of target DNA. Alternatively, the RDR6 produced dsRNA can be cleaved by DCL3, resulting in 24 nt siRNAs (Marí-Ordóñez et al., 2013). MicroRNA precursors or transcripts with inverted repeats can also be processed by $D C L 3$, and their resulting siRNAs activate the TGS pathway (Cuerda-Gil and Slotkin, 2016). The exact role for Ty-1 as RDRY in TGS, in the canonical or a non-canonical RNA directed DNA methylation pathway (Cuerda-Gil and Slotkin, 2016) remains to be determined.

Although a role of CBs in TGS is recognized, it has to be noted that the situation with CBs is complex. CBs also play a role in RNA metabolism, RNP formation and are involved in responses to disease and stress (Ding and Lozano-Durán, 2020; Love et al., 2017; Xu et al., 2020). CBs are very dynamic structures and constantly move around in the nucleus, while exchanging components with the nucleoplasm/ nucleolus (Morris, 2008; Xu et al., 2020). As a result, components of CB that are part of the TGS pathway are also observed elsewhere in the nucleus. AGO4 presents an example and is also found in AGO4/NRPD1b bodies (AB-bodies) which 
lack CB component Coilin (Li et al., 2008; Xu et al., 2020). Moreover, CB markers U2B" and Coilin not always fully colocalize and indicate the occurrence of distinct CB-like structures (Pontes et al., 2013). On this point, the observations made in this study, in which marker proteins Fib2 and U2B" do not fully co-localize during TYLCV infection, agree to these earlier findings. It is not unlikely that TYLCV infection enhances the dynamics of CB-like structures with distinct functions (Love et al., 2017; Xu et al., 2020).

In the absence of viral infection Ty-1 NBs localize juxtapose to COP1 photobodies, which contain photoreceptors (van Buskirk et al., 2012; Seo et al., 2004; Stacey and Von Arnim, 1999). COP1 regulates the photomorphogenic response and is a ubiquitin E3 ligase (Lau and Deng, 2012; Osterlund et al., 2000; Podolec and UIm, 2018; Saijo et al., 2003; Stacey et al., 1999; Yi and Deng, 2005). Ubiquitination is a PTM that affects the functionality, localization and/or turnover of proteins, but often targets the protein for proteasomal degradation. COP1 shuttles between the cytoplasm and nucleus in response to dark/light (von Arnim and Deng, 1994). In light, COP1 is present in the cytoplasm, while in the dark the protein moves to the nucleus where it targets certain transcription factors, involved in photomorphogenesis, for degradation (van Buskirk et al., 2012; Jang et al., 2005; Osterlund et al., 2000; Saijo et al., 2003; Seo et al., 2003; Yang et al., 2005). Earlier studies have shown a role of COP1 in the regulation of several dominant resistance genes as well in miRNA processing/RNAi (Cho et al., 2014; Jeong et al., 2010; Lim et al., 2018). HRT and RPM1, two dominant resistant genes of the NB-LRR type protecting against turnip crinkle virus (TCV) and the bacteria Pseudomonas syringae pv. tomato respectively, are positively regulated by COP1. Both resistance proteins interact with COP1 in the cytoplasm (Jeong et al., 2010; Lim et al., 2018), but the regulation of HRT appears indirect via double-stranded RNA binding proteins (DRBs) 1 and 4. Latter proteins stabilize the resistance protein and are protected from negative regulators by COP1 (Lim et al., 2018). Interestingly, DRBs also play a major role in RNAi as non-catalytic factors that promote dicing of dsRNA by DCLs (Muhammad et al., 2019). COP1 also inhibits a negative regulator of HYL1, a core protein in the processing of miRNA (Cho et al., 2014). The here described interaction between Ty-1 and COP1 only further strengthen the role of COP1 as regulator of plant immunity. Interestingly, it 
is the first time COP1 has been found to interact with a resistance gene in NBs. This interaction might lead to a light-dependent, negative or positive regulation of Ty-1.

Sumoylation is known to regulate plant defence responses on different levels (Verma et al., 2018). SIZ1 KO plants show upregulation of several disease response genes and SUMO1/SUMO2 knock down plants exhibit enhanced SA levels (van den Burg et al., 2010; Lee et al., 2006). In mammalian systems it has been shown that the sumoylation machinery is manipulated by viruses (Lowrey et al., 2017). In contrary, only very few cases have reported on sumoylation in relation to plant virus infections. Despite the absence of clear amino acid sequence homology to Ty-1, it is interesting to note that the turnip mosaic virus (TuMV) encoded viral RdRP is sumoylated and this promotes viral infection and suppresses the NPR1mediated resistance pathway (Cheng et al., 2017). NPR1 has also been shown to be sumoylated leading to its activation (Saleh et al., 2015). In addition, the TYLCVencoded Rep protein influences the sumoylation machinery. Rep interacts with SCE1 in NBs and changes the sumoylation status of specific host genes, and/or strongly induces higher expression levels of specific sumoylated host proteins to favour viral replication (Castillo et al., 2004; Maio et al., 2019; Sanchez-Duran et al., 2011). Whether Ty-1 is amongst those host genes and whether it localizes to the same NBs as Rep-SCE1 remains an intriguing question, considering that no changes in localization were observed during co-expression of Ty-1 and Rep.

The data presented in this study shows that upon TYLCV infection the expression of SCE1 and SIZ1 is increased and that only during infection these proteins aggregate into NBs. This is the first time that a regulation of SIZ1 by TYLCV is found. Previously it was observed that SUMO conjugation is the driving force for the formation of NBs with SUMO elements (Mazur et al., 2019). This suggests that TYLCV infection activates SUMO conjugation.

A recent report by Gou et al. showed a direct regulation of protein levels of the NBLRR resistance gene SNC1 by SIZ1 and that SNC1 is sumoylated (Gou et al., 2017). Although Ty-1 was found to interact with SUMO1, SUMO3, SIZ1 and SCE1, it is not yet known whether Ty-1 indeed is sumoylated or only interacts with sumoylated proteins. A prediction analysis performed on Ty- 1 revealed two potential SUMO 
attachment sites (SAS) (lysine residues K446 and K514) and three SIMs (Sup. Table 5.2). In order to enable their sumoylation respectively interactions with SUMO residues, these SAS and SIMs need to be accessible. A predicted folding structure of Ty-1 (Sup. Fig. 5.6A-C) revealed that K446 is expected to be embedded within the protein, whereas $\mathrm{K} 514$ is near the catalytic domain and possibly accessible. Of the three predicted SIMs, only the SIM at residue position 456 is predicted on the surface of the protein, altogether suggesting that Ty-1 could potentially become sumoylated as well as interact with sumoylated proteins. However, an alignment of all Arabidopsis encoded RDRs showed that none of the predicted sites are conserved in all RDRs (Sup. Fig. 5.6D). Residue K514 is conserved in all RDRs but only predicted to be sumoylated in RDR2 and Ty-1. This is interesting in light of the fact that Ty-1 and

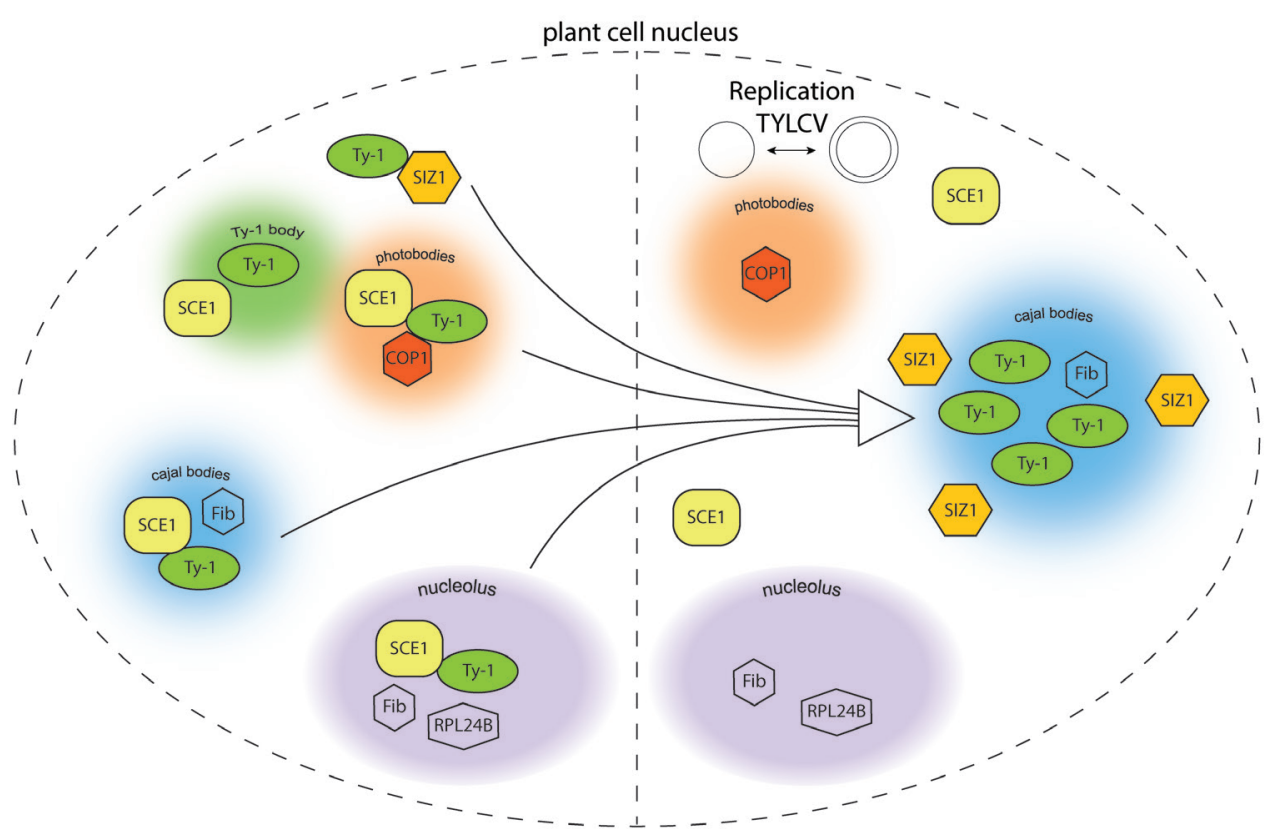

Figure 5.8: Schematic overview of the dynamic interplay of Ty-1 with COP1 and the sumoylation machinery and the localization of these elements prior and after viral infection.

Ty-1 primarily localizes neighbouring to COP1 containing photobodies in healthy cells, but during TYLCV infection relocalizes to cajal bodies. To indicate that the number of cajal bodies increases upon infection, the cajal body is drawn bigger in the situation of an infected plant cell. In uninfected cells, Ty-1 interacts with SCE1, SIZ1 and COP1 and these interactions seems to be decreased upon viral infections (observed via a decreased BIFC signal between Ty-1 and SCE1, SIZ1 and COP1). Upon infection, Ty-1 and SCE1 do not colocalize anymore in NBs and SIZ1 re-localizes into NBs nearby Ty-1 bodies. 
RDR2 both play a role in TGS. Further research is needed to indicate the biological significance of the predicted SAS and SIMs on the functionality of Ty-1.

In summary, this study has revealed a dynamic interplay between the $T y-1$ resistance gene encoded RDR, a key player of RNAi amplification, COP1 and the sumoylation machinery in NBs that changes during TYLCV infection, as visualized in Figure 5.8. How to explain these changes in localization and interactions and their relevance in light of the viral infection cycle and the Ty-1-mediated resistance response? One possibility is that Ty-1 becomes sumoylated for subsequent degradation, but only upon infection is prevented from this, leading to its stabilization and relocation to CBs to contribute to enhanced antiviral RNAi. Alternatively, Ty-1 only becomes sumoylated during viral infection for functional activation and re-localization to CBs. Another option is that Ty-1 is not sumoylated, but its interactions partners are, influencing Ty-1 complex formation and re-localization. Resolving the sumoylation status of Ty-1 in the presence or absence of viral infection will help to clarify this issue. In conclusion, this data shows the interplay of an atypical resistance gene and the sumoylation machinery, giving the first clues for the importance of this PTM in regulating TGS as antiviral defence response.

\section{Experimental procedures}

\section{Plants and viruses}

Nicotiana benthamiana plants were maintained under climate chamber conditions $\left(22{ }^{\circ} \mathrm{C}, 16 \mathrm{~h}\right.$ light/8 h dark, 70\% relative humidity). S. lycopersicum plants were maintained under greenhouse conditions $\left(23^{\circ} \mathrm{C}\right.$ during the day, $21^{\circ} \mathrm{C}$ at night, $16 \mathrm{~h}$ light/8 h dark, 60\% relative humidity). S. Iycopersicum cv. MM was used and a Ty-1 introgression line was derived from S. chilense LA1969 (Verlaan et al., 2013). For TYLCV infection, an agro-infectious clone of TYLCV Israelian strain isolated from Almeria, Spain, as described by Morilla et al. (2005) (GenBank AJ489258.1) was used. For infection, the agroinfiltration protocol as described under protein expression was followed. 


\section{Binary vectors for protein expression}

The binary vector pK7WG2 expressing Ty-1, as described in Chapter 2, was used as backbone to amplify the coding sequence of Ty-1 via a PCR with primers attb-Ty1-F and attb-Ty1-R and using Phusion high-fidelity polymerase (Thermo Scientific) (See Sup. Table 5.1 for primer sequences). The PCR product was cloned into Pjet1.2 cloning vector according to the manufacturer's protocol (ThermoScientific) and from there transferred to the gateway vector pDONR207 via a BP reaction using BP clonase (Invitrogen). Subsequently, Ty-1 CDS was moved via a LR reaction using LR clonase to the destination vector pK7WGF2 for N-terminal fusion to enhanced green fluorescent protein (GFP) or to the destination vector PDEST-SCYNE(R) ${ }^{\mathrm{GW}}$ for $\mathrm{N}$-terminal fusion to the $\mathrm{N}$-terminal subfragment of super cyan fluorescent protein (SCFP). The CDS of the viral proteins were amplified from the TYLCV infectious clone ((Morilla et al., 2005)(GenBank AJ489258.1)) via PCR using primers attb-V1-F and attb-V1-R, attb-V2-F and attb-V2-R, attb-C1-F and attb-C1-R, attb-C2-F and attb-C2-R, attb-C3-F and attb-C3-R, or attb-C4-F and attb-C4-R, to amplify V1, V2, $C 1, C 2, C 3$ and C4 CDS respectively. A vector containing the CDS of HYPONASTIC LEAVES 1 (HYL1) was ordered from The Arabidopsis Information Resource (TAIR), and from there the CDS was amplified by PCR using primers attb-HYL1-F and attb-HYL1-R. Vector pROK2-U2B"-GFP, provided by Michael Taliansky, was used as template to amplify the CDS of U2B" using a PCR with the primers attb-U2B"-F and attb-U2b"-R. To clone the CDS of SIZ1, SCE1, SUMO1 and SUMO3, first RNA was extracted using TRIzol reagent and subsequently first-strand synthesis was performed using M-MLV reverse transcriptase (Promega) and random hexamers (Roche) following manufacturers protocol. The CDS were amplified using primer pairs attb-SIZ1-F and attb-SIZ1-R, attb-SCE1-F and attb-SCE1-R, attb-SUMO1-F and attb-SUMO1-R, attb-SUMO3-F and attb-SUMO3-R, to amplify SIZ1, SCE1, SUMO1 and SUMO3 CDS respectively. All the PCR amplicons were transferred via a BP reaction to the Gateway vector pDONR207 using BP Clonase (Invitrogen). The construct pEarleyGate104 containing the CDS of COP1 was kindly provided by Dongqing Xu (Xu et al., 2015). From this construct, the COP1 CDS was transferred to pDONR207 via a BP reaction. From the pDONR207 vector, the CDS of HYL1, U2B", all viral proteins, SCE1, SIZ1, SUMO1 and SUMO3 were transferred via a LR reaction using LR clonase (Invitrogen) to the destination vector pGWB455 and the CDS of 
COP1 to the destination vector pGWB655 for N-terminal fusion to monomeric red fluorescent protein (mRFP1) (Nakagawa et al., 2007; Nakamura et al., 2010). The CDS of COP1, SIZ1, SCE1, SUMO1 and SUMO3 were also transferred from the pDONR207 vector to the destination vector PDEST-SCYCE $(R)^{\mathrm{GW}}$ for $\mathrm{N}$-terminal fusion to the C-terminal sub-fragment of SCFP. All vectors were checked via restriction digestion for expected digestion pattern and sequenced (Eurofins). All primers were synthesized by Integrated DNA Technologies. Vectors expressing mRFP tagged AtFib2 (pROK2-AtFib2-mRFP) and mCherry tagged AtRPL24B (pEarly-gate101 based vector, called RFP-AtRPL24B) were described in Martinez et al (2014) (Kim et al., 2007; Martinez and Daros, 2014). Vector pGDR-NbFib2 expressing DsRed tagged NbFib2 (called RFP-NbFib2) was described by Duan et al. (2012). To express GFP tagged U2B" the vector pROK2-U2B"-GFP, kindly provided by Michael Taliansky, was used. P19 was expressed from vector pBin19, which was described earlier (Hedil et al., 2015).

\section{Transient expressions of proteins}

Proteins were transiently expressed via an Agrobacterium Transient Transformation assay (ATTA). Agrobacterium tumefaciens (A. tumefaciens) strains LBA4404 or GV3101 were transformed with the destination plasmids via electroporation. Either the protocol as described in Mazur et al. (2019) or as described in Chapter 2 was followed to perform the ATTAs. A. tumefaciens cells were infiltrated by pressure inoculation with a needle-less syringe into the abaxial side of 4- to 5-week-old $\mathrm{N}$. benthamiana leaves with an OD600 of 0.5 per construct. All infiltrations were performed in the presence of GV3101 A. tumefaciens containing pBIN expressing $\mathrm{P} 19$ (OD600=0.2) (Hsieh et al., 2009). When protein localization was examined in the presence of TYLCV, a co-infiltration was performed with $A$. tumefaciens strain LBA4404 containing the agro-infectious clone $(O D 600=0.5)$. Protein accumulation was examined 3 days post infiltration (dpi). TYLCV infection in tomato plants was performed according to the same protocol and in that case the first two true leaves were fully infiltrated on the abaxial side of the leaf. 


\section{Western blot analysis}

To detect transiently expressed proteins in $N$. benthamiana leaves, the protocol as described by Mazur et al. (2019) was used with minor modifications. In brief, the infiltrated area of $N$. benthamiana leaf was snap frozen in liquid nitrogen and subsequently a total protein extraction was performed by grinding the leaf in $2 X$ (v/w) extraction buffer (8 M urea, $100 \mathrm{mM}$ Tris, pH 6.8, 2\% [w/v] SDS, $10 \mathrm{mM}$ dithiothreitol). The sample was incubated on ice for $15 \mathrm{~min}$, centrifuged at $13.000 \mathrm{~g}$ for $20 \mathrm{~min}$ at $4{ }^{\circ} \mathrm{C}$, the supernatants was transferred to new tubes and the same volume of $2 x$-SDS-loading buffer was added. After incubation at $95^{\circ} \mathrm{C}$ for 3 minutes, proteins were separated on $10 \%(\mathrm{w} / \mathrm{v})$ SDS-PAGE, and then blotted to nitrocellulose membranes (Immobilon P). The membrane was blocked with $1 \%(\mathrm{w} / \mathrm{v})$ milk in phosphate-buffered saline containing $0.05 \% \mathrm{v} / \mathrm{v}$ Tween. Subsequently, GFP tagged proteins were detected using polyclonal rabbit anti-GFP antibodies (Invitrogen, 1:2000). Goat-anti-Rabbit conjugated to alkaline phosphatase (Dako, 1:2000) was used as secondary antibody. The proteins were visualized using NBT-BCIP (Roche) and detecting the colorimetric reaction.

\section{Immuno-precipitation and detection via Mass spectrometry}

Infiltrated leaf area was harvested $3 \mathrm{dpi}$ and snap-frozen in liquid nitrogen. After grinding in liquid nitrogen, extraction buffer $(150 \mathrm{mM} \mathrm{NaCl}, 50 \mathrm{mM}$ Tris, $\mathrm{pH} 8.0$, $1 \%$ NP-40, protease inhibitor cocktail (Sigma, 1 tablet in $50 \mathrm{ml}$ )) was added to the sample in $1 \mathrm{gram}: 2 \mathrm{ml}$ ratio and the sample was incubated on ice until thawed. After centrifugation for $30 \mathrm{~min}, 34.000 \mathrm{~g}$ at $4{ }^{\circ} \mathrm{C}$, GFP-Trap magnetic agarose beads (chromotek), washed with extraction buffer, were added to the supernatant and incubated 1 hour at $4{ }^{\circ} \mathrm{C}$. Beads were washed 3 times with extraction buffer, using a magnetic holder. The beads were resuspended in protein denaturation buffer and supernatant was loaded on a $12 \%$ bisacrylamide gel. A band of 50kDa-250kDA was cut out and the sample was prepared for LC-MSMS as previously described with minor modifications (Jashni et al., 2015). In brief, first proteins were fixed by incubation in $5 \%$ acetic acid/45\% water $/ 50 \%$ methanol (v/v/v). The gel band was cut in small pieces and washed with $50 \mathrm{mM}$ ammonium bicarbonate. Subsequently, 
for cysteine reduction and alkylation, the gel pieces were incubated in $10 \mathrm{mM}$ dithiothreitol in $50 \mathrm{mM}$ ammonium bicarbonate for 1 hour at $60^{\circ} \mathrm{C}$, followed by incubation in $15 \mathrm{mM}$ iodoacetamide in $50 \mathrm{mM}$ Tris $\mathrm{pH}$ 8. After washing the gel pieces twice with $50 \mathrm{mM}$ ammonium bicarbonate proteins were enzymatically digested by adding $5 \mathrm{ng} / \mu \mathrm{l}$ sequencing-grade bovine trypsin (Roche) in $50 \mathrm{mM}$ ammonium bicarbonate and incubating for 2 hours at $45^{\circ} \mathrm{C}$. To extract the peptides, the gel piece was sonicated and $10 \%$ trifluoroacetic acid (TFA) in $50 \mathrm{mM}$ ammonium bicarbonate was added to adjust to $\mathrm{pH} 3$. Any remaining gel pieces were removed from the sample by running it over a home-made column containing C18 empore disk paper. The resulting peptides were analysed by liquid chromatography-tandem mass spectrometry (LC-MSMS). The data files from the LTQ-Orbitrap were analyzed with MaxQuant software. The default settings were used, except that deamidation of asparagine and glutamine were added as variable modifications. To identify GFP tagged Ty-1 proteins, the obtained MS-MS spectra were searched against the protein sequence of Ty-1.

\section{Confocal Microscope}

$N$. benthamiana leaf material was analysed $3 \mathrm{dpi}$. GFP, RFP and SCFP signal in leaf epidermal cells was detected with a confocal laser scanning microscope (Zeiss LSM 510-META 18) using the Plan-Apochromat 63x oil immersion objective. For all samples, the bright field images were collected via the Differential Interference Contrast (DIC) channel and pinhole was set to 1. For GFP imaging, an argon laser was used (Ex: 488nm). The emitted light passed the dichroic beam splitter 488/543, was reflected by the secondary dichroic beam splitter 545 and then passed the band pass 505-530, upon which the signal was detected. For RFP imaging, an helium neon laser (Ex: $543 \mathrm{~nm}$ ) was used for excitation. The emitted lights passed the dichroic beam splitter $488 / 543$ and then the light passed a secondary dichroic beam splitter 490, followed by a band pass 560-615, upon which the signal was detected. For SCFP imaging, an argon laser was used (Ex: 458). The emitted lights passed the dichroic beam splitter 458 and then the light was reflected by a secondary dichroic beam splitter 545 , followed by a band pass $470-500$, upon which the signal was detected. When multiple fluorophores were imaged in one sample, sequential scan setting 
was used. The obtained images were processed with the software program ImageJ. For each observation, at least two independent agro-infiltrations and subsequent imaging were performed

\section{Gene expression analysis}

RNA was isolated from systemic tomato leaves using the MirVana miRNA isolation kit (Invitrogen), following the manufacturer's instructions. The purified RNA was treated with TURBO DNAse (Invitrogen) according to the manufacturer's protocol. Oligo d(T) primers (IDT) and M-MLV reverse transcriptase were used according to the manufacturers' protocol (Promega) to synthesize first strand cDNA. The RT-qPCR was performed in a total volume of $10 \mu \mathrm{L}$, containing $3 \mu \mathrm{L}$ of $10 x$ diluted cDNA, 1x Sybr Select (Applied Biosystems), $375 \mathrm{nM}$ forward primer and $375 \mathrm{nM}$ reverse primer, in a technical duplicate. The primer pairs COP1-1F and COP1-1R, COP1-2F and COP1-2R, SCE1-1F and SCE1-1R, SCE1-2F and SCE1-2R, SCE1-3F and SCE1-3R, SCE1-4F and SCE1-4R, SIZ1-1F and SIZ1-1R, SIZ1-2F and SIZ1-2R and Fib-F and Fib-R were used to measure the expression of COP1 homologs Solyc11g011980 and Solyc12g005950, SCE1 homologs Solyc04g078620, Solyc12g088680, Solyc02g093110 and Solyc03g044260, SIZ1 homologs Solyc06g010000 and Solyc11g069160 and Fib2 homolog Solyc03g025270 respectively (for primer sequences see Sup. Table 5.1). These homologous genes in tomato were determined via sequence homology between the coding sequences of the genes in Arabidopsis thaliana and the tomato CDNA database (ITAG release 2.40), and via homologs annotated in the PANTHER database. The gene expression was measured relative to the average expression of the housekeeping genes EXP, GAPDH and UK by using the primer pairs EXP-F and EXP-R, GAPDH-F and GAPDH-R and UK-F and UK-R respectively. The RT-qPCR was performed in a Bio-Rad CFX96. Relative gene expression was calculated using the DeltaDeltaCt method (Livak and Schmittgen, 2001). Values were normalized relative to the housekeeping genes, and calibrated to levels of the control plants (MM uninfected), which were set as 1 . 


\section{Protein predictions}

SUMO attachment sites (SAS) and SIMs were predicted using the GPS sumoylation prediction server (high threshold for sumyolation and SUMO interaction) and the Jassa server (only "high cut-off" results for predicted SIMs). The protein structure of Ty-1 was predicted using the Protein Homology/analogY Recognition Engine $\mathrm{V}$ 2.0 (Phyre2 web portal), using the intensive modelling mode (Kelly et al., 2015). An alignment of the amino acid sequences of RDR1 to -6 and Ty- 1 was performed using Clustal Omega.

\section{Acknowledgements}

The authors thank Yuling Bai (Wageningen University, Wageningen) for providing vector pK7WG2-Ty-1 and for discussion. We thank Michael Talianksy (The James Hutton Institute, Dundee), José-Antonio Daròs (Institutio de Biología Molecular y Celular de Plantas, Valencia), Dongqing Xu (Peking University, Beijing), Hui-Shan Guo (University of California, California) and Eduardo Rodríguez Bejarano (Universidad de Málaga, Malaga, Spain) for providing several plasmids. The authors kindly acknowledge S. Boeren (Wageningen University and Research, Wageningen) for assistance with the MS measurement and data analysis and N. de Ruijter from the Wageningen Light Microscopy Centre for assistance in cellular imaging. This research was financially supported by NWO-CNPq within the Joint Research Project Biobased Economy (729.004.011). 


\section{Supplemental data}

A.

mRFP-V1

mRFP-V2

MRFP-C1

mRFP-C2

mRFP-C3

mRFP-C4

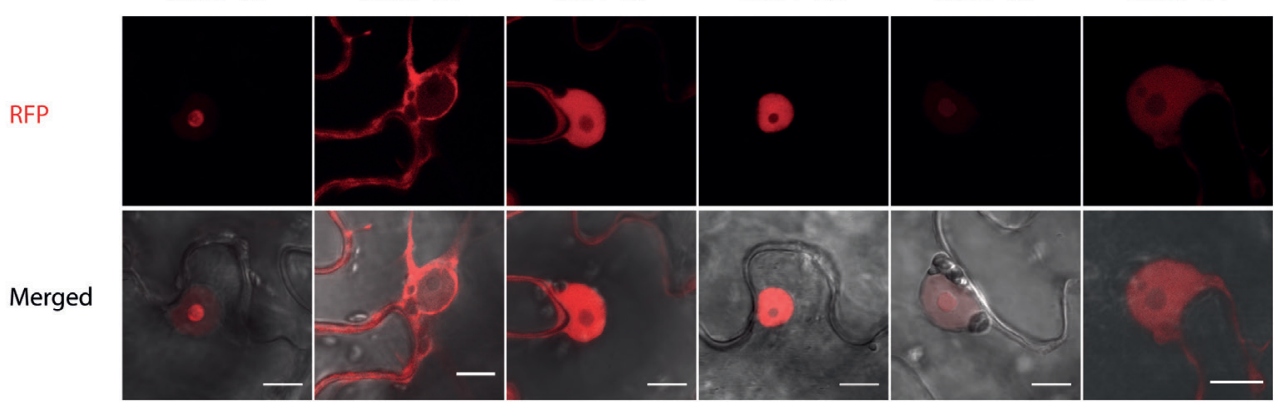

B.

GFP-Ty-1

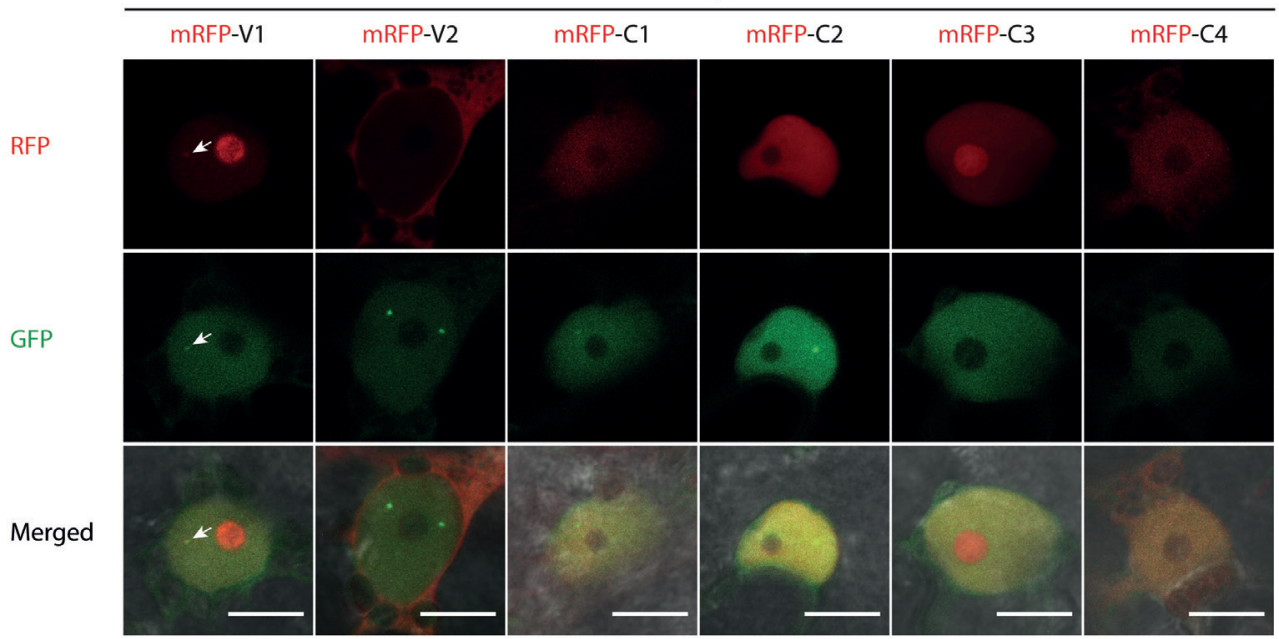

Supplemental Figure 5.1: TYLCV encoded proteins do not influence the localization of Ty-1 similarly to viral infection.

A. Viral proteins were transiently expressed as RFP fusion proteins in $N$. benthamiana cells using agroinfiltration and visualised $3 \mathrm{dpi}$ using the confocal microscope. The scale bars represent $10 \mu \mathrm{m}$. B. Co-expression of Ty-1 fused to GFP and viral proteins fused to RFP. Fusion proteins were transiently expressed in $N$. benthamiana cells using agroinfiltration and visualised $3 \mathrm{dpi}$ using the confocal microscope. The arrows indicate co-localization of GFP-Ty-1 and RFP-V1. The scale bars represent $10 \mu \mathrm{m}$. 


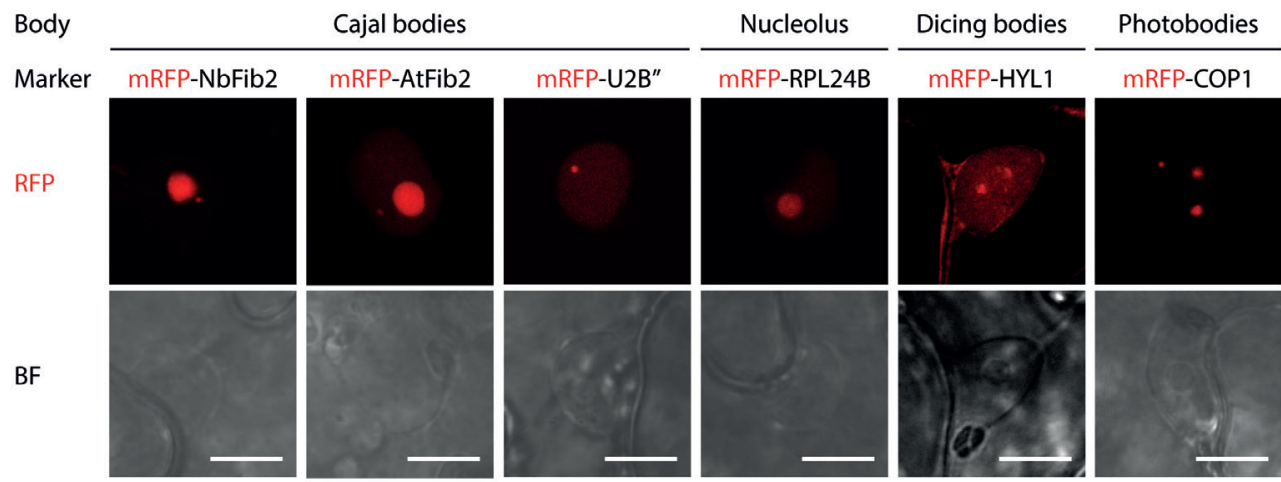

Supplemental Figure 5.2: The localization of RFP tagged marker proteins of nuclear bodies.

Marker proteins of several nuclear bodies fused to RFP were transiently expressed in $N$. benthamiana cells using agroinfiltration and analysed $3 \mathrm{dpi}$ using the confocal microscope. RFP tagged markers for CBs (NbFib2, AtFib2, U2B"), nucleolus (RPL24B), photobodies (COP1) and dicing bodies (HYL1) were analysed. The scale bars represent $10 \mu \mathrm{m}$.

GFP-U2B"

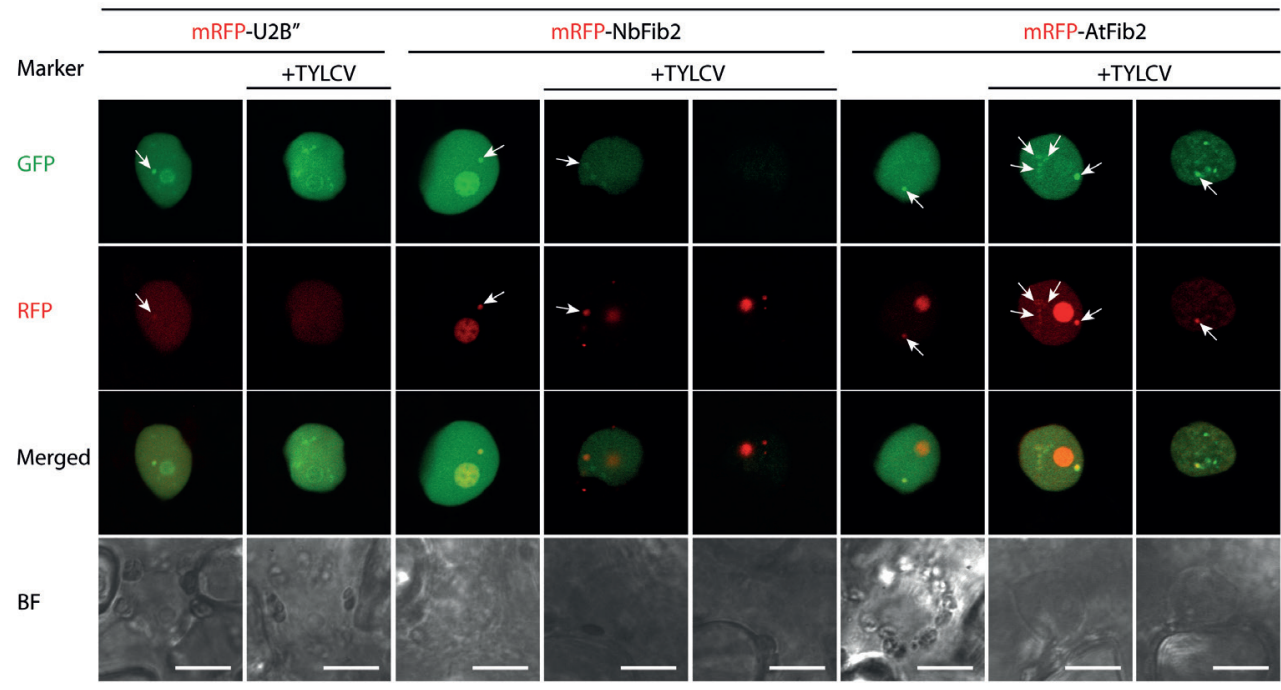

Supplemental Figure 5.3: Upon infection with TYLCV, the number of CBs increase.

U2B" fused to GFP was co-expressed with U2B", NbFib2 or AtFib2 respectively fused to RFP in the presence or absence of TYLCV via agroinfiltration. The $N$. benthamiana leaves were co-infiltrated with the constructs and analysed $3 \mathrm{dpi}$. The arrows indicate the co-localized signal of GFP-U2B" and RFP tagged CB markers. The scale bars represent $10 \mu \mathrm{m}$. 

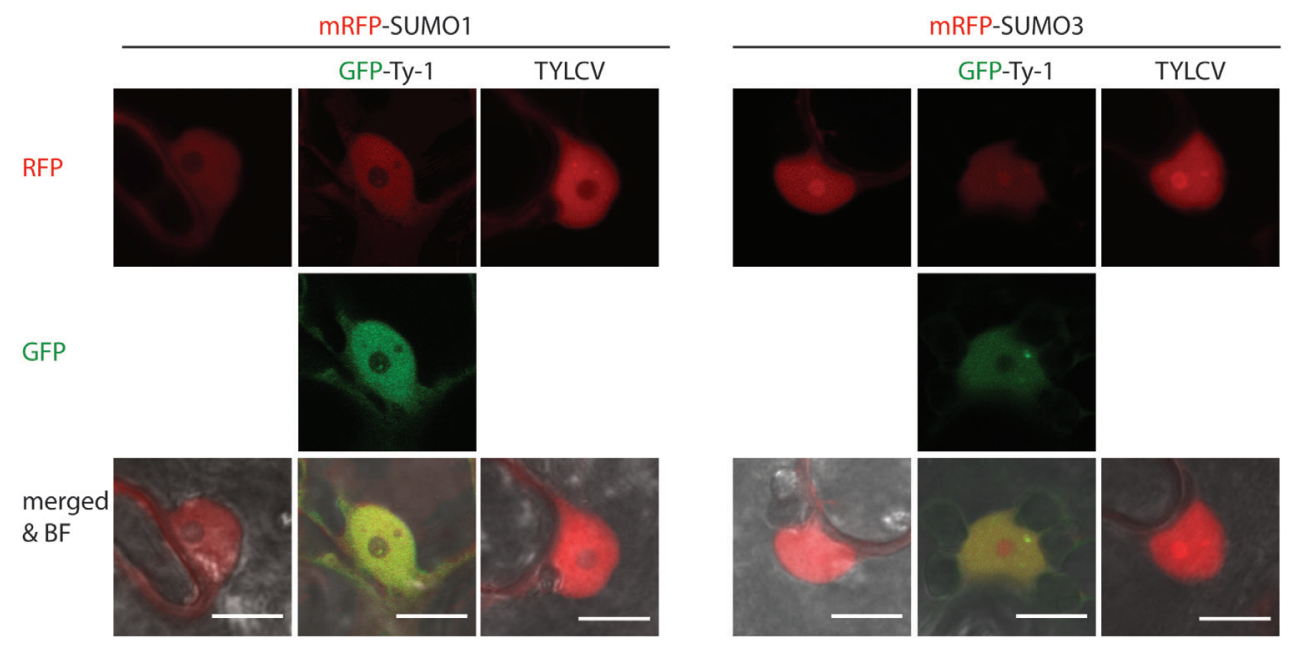

Supplemental Figure 5.4: Ty-1 co-localizes with SUMO3 and TYLCV infection induces NB formation of SUMO1 and SUMO3.

Fusion proteins of SUMO1 and SUMO3 to RFP were either expressed alone or together with Ty-1 fused to GFP or together with an infectious clone of TYLCV in N. benthamiana leaves. The localization was analysed $3 \mathrm{dpi}$ using the confocal microscope. The scale bars represent $10 \mu \mathrm{m}$.

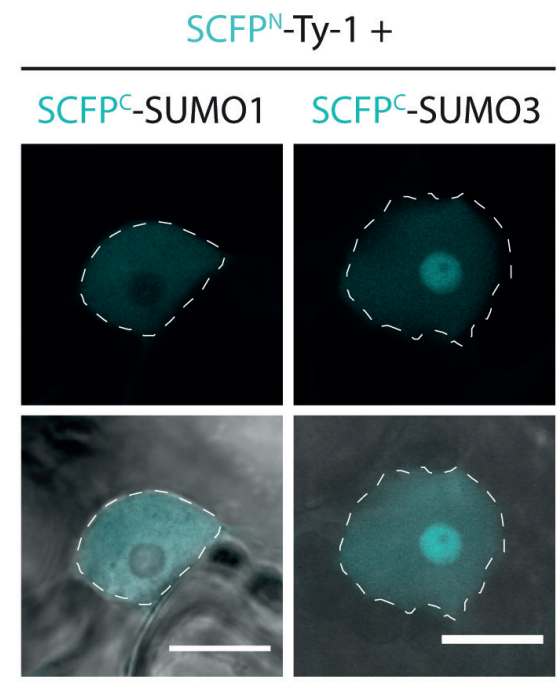

Supplemental Figure 5.5: Ty-1 interacts with SUMO1 and SUMO3.

Ty-1 was expressed as fusion protein with the $\mathrm{N}$-terminal half of SCFP (SCFPN), together with either SUMO1 or SUMO3 as fusion protein with the C-terminal half of SCFP (SCFPC) in N. benthamiana cells via ATTA and the reconstituted CFP signal imaged $3 \mathrm{dpi}$ using the confocal microscope. The scale bars represent $10 \mu \mathrm{m}$. 


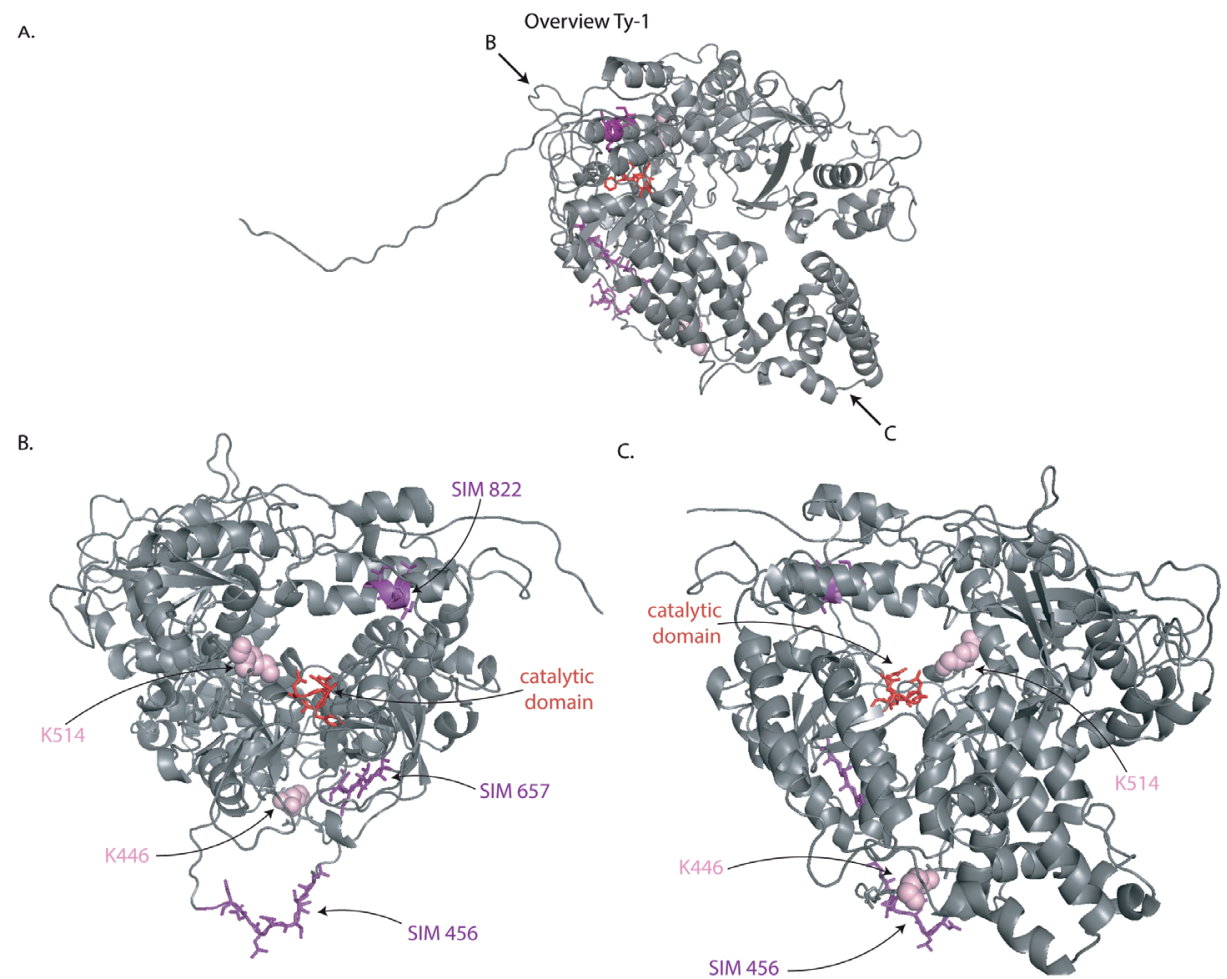

D.

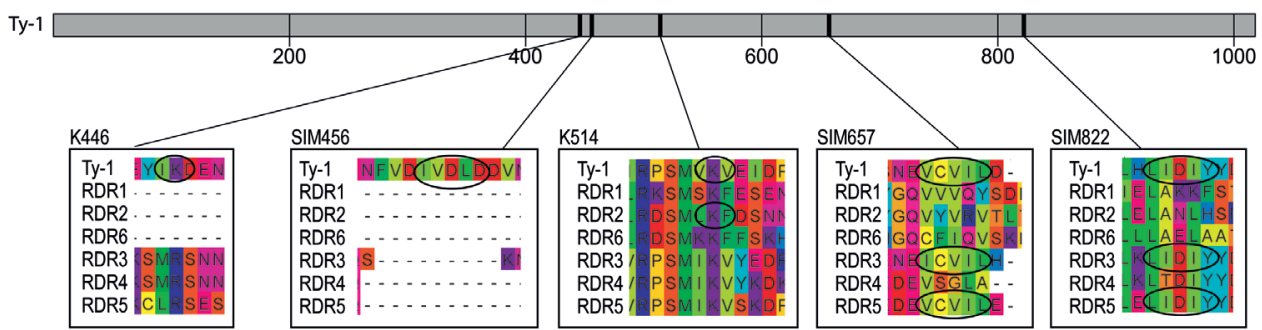

Supplemental Figure 5.6: Ty-1 contains predicted SAS and SIM of which some are conserved in other RDRs. A-C. The folding of Ty-1 was predicted using the Phyre2 server. In the folded protein the predicted SAS (Lysine residues in pink), SIMs (in purple) and catalytic domain (in red) are depicted. The folded protein is depicted from different angles (Fig. a, b, c) to show all SAS and SIM sites. D. Ty-1 was aligned with all 6 RDRs encoded by Arabidopsis thaliana and the predicted SAS and SIM sites predicted in Ty-1 are indicated to be present or absent in the other proteins (according to the GPS sumoylation prediction server and the Jassa server, depicted with a circle if present). 
Ty-1 localizes in nuclear bodies and interplays with the sumolation machinery

Supplemental Table 5.1: A list of all primer sequences used in this study.

\begin{tabular}{|c|c|}
\hline Name & Sequence 5'to 3' \\
\hline$a t t b-C 1-F$ & $\begin{array}{l}\text { GGGG ACA AGT TTG TAC AAA AAA GCA GGC TCA ATG CCT CGT TTA TTT AAA } \\
\text { ATA TAT GC }\end{array}$ \\
\hline$a t t b-C 1-R$ & $\begin{array}{l}\text { GGGG AC CAC TTT GTA CAA GAA AGC TGG GTC TTA CGC CTT ATT GGT TTC TTC } \\
\text { TTG G }\end{array}$ \\
\hline$a t t b-C 2-F$ & GGGG ACA AGT TTG TAC AAA AAA GCA GGC TCAATGCAACCTTCGTCACCC \\
\hline$a t t b-C 2-R$ & $\begin{array}{l}\text { GGGG AC CAC TTT GTA CAA GAA AGC TGG GTCCTAAATACTCTTAAGAAATGAC- } \\
\text { CAGTCTG }\end{array}$ \\
\hline$a t t b-C 3-F$ & GGGG ACA AGT TTG TAC AAA AAA GCA GGC TCAATGGATTCACGCACAGGG \\
\hline$a t t b-C 3-R$ & $\begin{array}{l}\text { GGGG AC CAC TTT GTA CAA GAA AGC TGG GTCTTAATAAAATTTATATTTTATATCAT- } \\
\text { GAGTTTCTGTTAC }\end{array}$ \\
\hline$a t t b-C 4-F$ & GGGG ACA AGT TTG TAC AAA AAA GCA GGC TCAATGGGGAACCACATCTCC \\
\hline$a t t b-C 4-R$ & GGGG AC CAC TTT GTA CAA GAA AGC TGG GTCTTAATATATTGAGGGCCTCGG \\
\hline$a t t b-H Y L 1-F$ & GGGGACAAGTTTGTACAAAAAAGCAGGCTCAATGACCTCCACTGATG \\
\hline$a t t b-H Y L 1-R$ & GGGGACCACTTTGTACAAGAAAGCTGGGTCTTATGCGTGGCTTGC \\
\hline$a t t b-S C E 1-F$ & GGGGACAAGTTTGTACAAAAAAGCAGGCTCCATGGCTAGTGGAATCGCT \\
\hline$a t t b-S C E 1-R$ & GGGGACCACTTTGTACAAGAAAGCTGGGTATTAGACAAGAGCAGGATACTG \\
\hline$a t t b-S I Z 1-F$ & GGGGACAAGTTTGTACAAAAAAGCAGGCTCAATGGATTTGGAAGCTAATTGTAAGGA \\
\hline$a t t b-S I Z 1-R$ & GGGGACCACTTTGTACAAGAAAGCTGGGTCCTCAGAATCCGAGTCAATGGAGAGG \\
\hline$a t t b-S U M O 1-F$ & $\begin{array}{l}\text { GGGGACAAGTTTGTACAAAAAAGCAGGCTCAATGTCTGCAAACCAGGAGGAAGA- } \\
\text { CAAG }\end{array}$ \\
\hline$a t t b-S U M O 1-R$ & GGGGACCACTTTGTACAAGAAAGCTGGGTATCAGCCACCAGTCTGATGGAG \\
\hline$a t t b-S U M O 3-F$ & $\begin{array}{l}\text { GGGGACAAGTTTGTACAAAAAAGCAGGCTCAATGTCTAACCCTCAAGATGACAAGC- } \\
\text { CCATC }\end{array}$ \\
\hline$a t b-S U M O 3-R$ & GGGGACCACTTTGTACAAGAAAGCTGGGTATCAACCACCACTCATCGCCCGGCAC \\
\hline$a t b-T y 1-F$ & $\begin{array}{l}\text { GGGGACAAGTTTGTACAAAAAAGCAGGCTTCGAAGGAGATAGAACCATGGGTGATC- } \\
\text { CGTTGATTGAAGAAATTG }\end{array}$ \\
\hline$a t t b-T y 1-R$ & GGGGACCACTTTGTACAAGAAAGCTGGGTCCTAGAGTATTTCCTGCAAAACCGATG \\
\hline$a t t b-U 2 B^{\prime \prime}-F$ & GGGGACAAGTTTGTACAAAAAAGCAGGCTCAATGTTAACGGCAG \\
\hline$a t t b-U 2 B^{\prime \prime}-R$ & GGGGACCACTTTTGTACAAGAAAGCTGGGTCTCATTTCTTGGCGAAAG \\
\hline$a t t b-V 1-F$ & GGGG ACA AGT TTG TAC AAA AAA GCA GGC TCAATGTCGAAGCGACCAGGC \\
\hline$a t t b-V 1-R$ & $\begin{array}{l}\text { GGGG AC CAC TTT GTA CAA GAA AGC TGG GTCTTAATTTGATATTGAATCATA- } \\
\text { GAAATAGATGCG }\end{array}$ \\
\hline$a t t b-V 2-F$ & GGGG ACA AGT TTG TAC AAA AAA GCA GGC TCAATGTGGGACCCACTTCTAAATG \\
\hline$a t t b-V 2-R$ & GGGG AC CAC TTT GTA CAA GAA AGC TGG GTCTCAGGGCTTCGATACATTCTG \\
\hline COP1-1F & GCGCTATTGGGCAAGACAGT \\
\hline
\end{tabular}




\begin{tabular}{|c|c|}
\hline COP1-1R & AATCCTCGAGTCCCGCAGAA \\
\hline COP1-2F & GCTGGAGTTTCACGGCGTAT \\
\hline COP1-2R & ACAACAGGGCAGTGTGCATC \\
\hline$E X P-F$ & GCTAAGAACGCTGGACCTAATG \\
\hline$E X P-R$ & TGGGTGTGCCTTTCTGAATG \\
\hline$F i b-F$ & ACCAGCTGAAGCTGTGTTTGC \\
\hline$F i b-R$ & AGGCACCAACAACACAAGCG \\
\hline GAPDH-F & ACCACAAATTGCCTTGCTCCCTTG \\
\hline$G A P D H-R$ & ATCAACGGTCTTCTGAGTGGCTGT \\
\hline SCE1-1F & CGGAGACTGGTCCTGATGGT \\
\hline SCE1-1R & ACTGGATAGTGACCGCCCTC \\
\hline SCE1-2F & AACCGCCCAAGTGCAAGTTT \\
\hline SCE1-2R & GCCTCCACCCACTGTCTTCA \\
\hline SCE1-3F & TCCGAGTAAACCGCCAAAGTG \\
\hline SCE1-3R & GTTTCACGGTGATGGCTGGT \\
\hline SCE1-4F & AGCTTGGCGCAGGAATCATC \\
\hline SCE1-4R & AGCAGTGCCACACCATCAAG \\
\hline SIZ1-1F & CGGCGTCGTACTCTCGTACA \\
\hline$S I Z 1-1 R$ & TTTCAGTGGCAGTTCCCCCA \\
\hline$S I Z 1-2 F$ & GGAGGGGTTCCTGGTGATAC \\
\hline$S I Z 1-2 R$ & CATACCAATAAGCAATGAAGCAGTG \\
\hline$U K-F$ & TGGTAAGGGCACCCAATGTGCTAA \\
\hline$U K-R$ & ATCATCGTCCCATTCTCGGAACCA \\
\hline
\end{tabular}


Supplemental Table 5.2: Ty-1 is predicted to contain SUMO attachment sites and SUMO interaction motifs.

\begin{tabular}{|c|c|c|c|}
\hline \multicolumn{4}{|c|}{ Predicted SAS } \\
\hline Position & Peptide & P-value ${ }^{\#}$ & Predictive score* \\
\hline $313^{*}$ & FFVYKDDKERKKSPAMMKTKT & & Low \\
\hline $446^{\# *}$ & FSKAEYIKDENYENF & 0.003 & High \\
\hline $514^{\# *}$ & HIRPSMVKVEIDPTI & 0.007 & High \\
\hline $623^{*}$ & HLHVRLSRLAKIERTKLRGGK & & Low \\
\hline $914^{*}$ & TLWRGRYEEYKKDMTQAMNLD & & Low \\
\hline \multicolumn{4}{|c|}{ Predicted SIM } \\
\hline Position & Peptide & P-value & Predictive score* \\
\hline $456-460^{\# *}$ & NYENFVDIVDLDDVNVERR & 0.051 & 7.140 \\
\hline $657-661^{\#}$ & GVLESNEVCVILDNGQVSG & 0.04 & \\
\hline $822-825 *$ & SLKGKMLHLIDIYYDALDAP & & 3.119 \\
\hline
\end{tabular}

SUMO attachment sites (SAS) and SUMO Interaction Motifs (SIMs) were predicted using either the GPS sumoylation server or the Jassa prediction server. The results of these predictions are depicted here, including the p-value (GPS sumoylation server) and predictive scores (Jassa prediction server).

\# Predicted with GPS Sumo server

* predicted with Jassa prediction server 


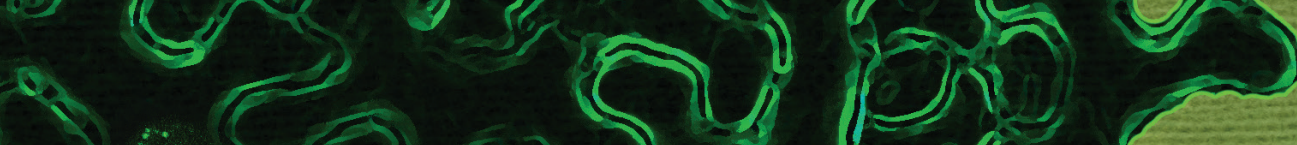

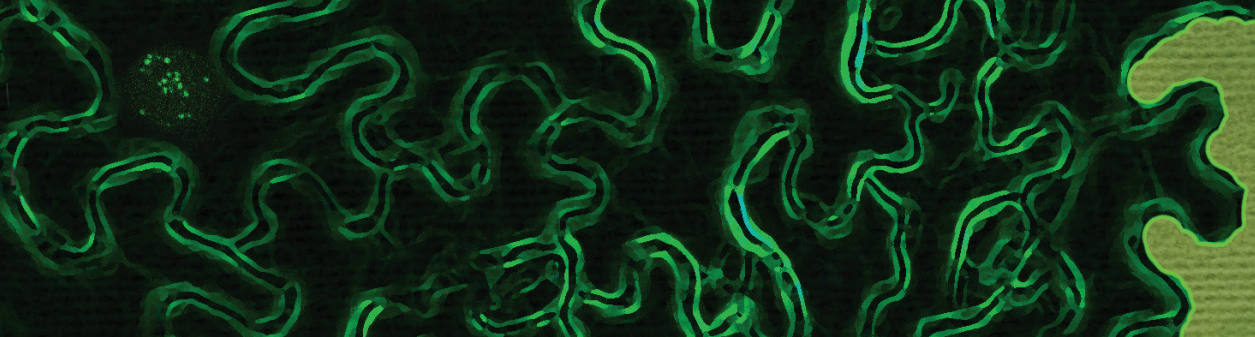

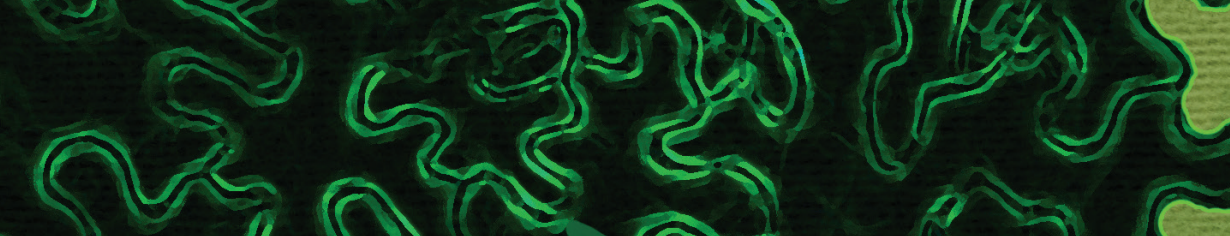

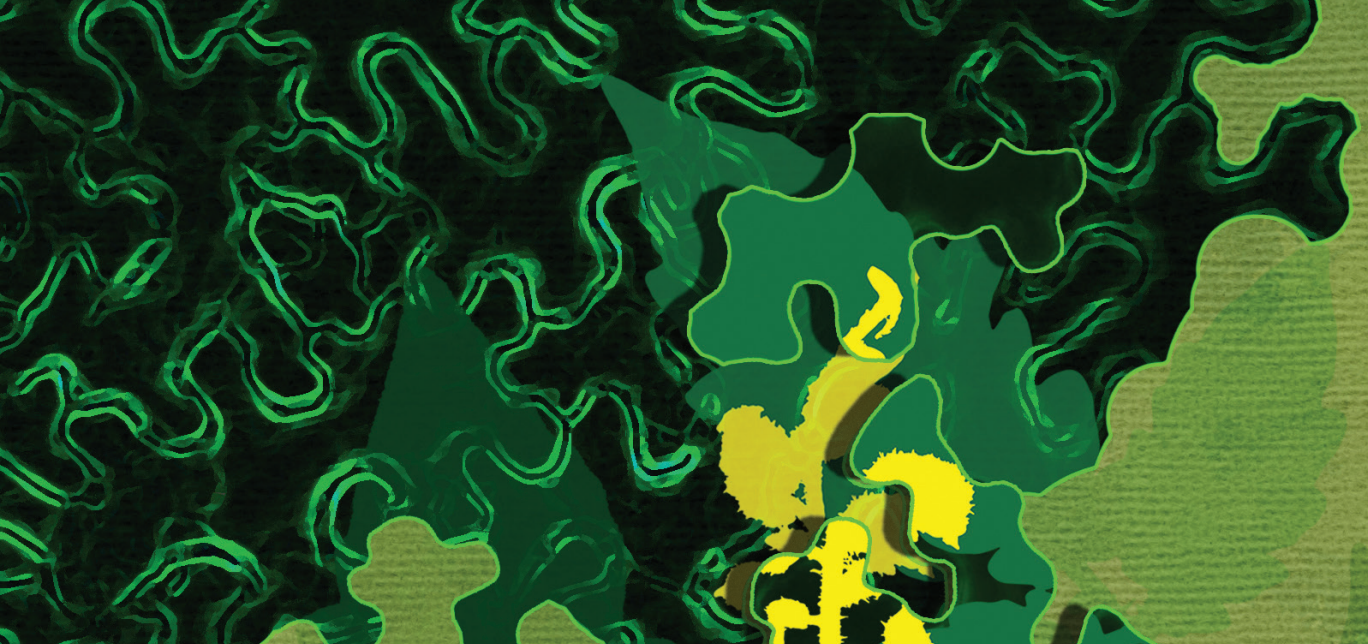

- $\Omega$. a $25,5=0$

) कर दर:? (2) 25?

$0 \sqrt{1}$

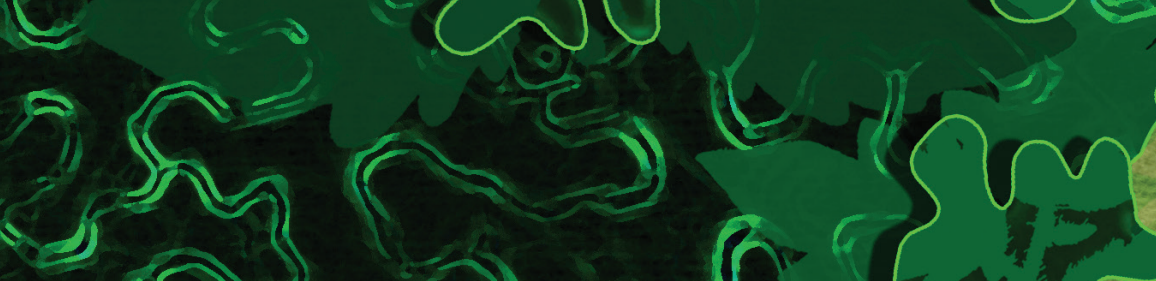

53 a n (1)

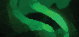
2 


\section{Chapter 6}

\section{General discussion}

C.M. Voorburg 
This thesis describes a study on the working mechanism behind $T y-1$, one of the available and first cloned resistance genes against tomato yellow leaf curl virus (TYLCV). A previous study already showed that the presence of Ty-1, a member of the RNA-dependent RNA polymerase (RDR) y class, increases the abundance of viral small interfering RNAs (vsiRNAs) and the level of viral DNA methylation, indicating a role in the amplification of the TGS response. Data presented in this thesis show that the resistance spectrum of $T y-1$ is not limited to TYLCV and other (whitefly-transmitted) begomoviruses, but also extends to curtoviruses, another genus within the Geminiviridae and whose members are transmitted by leafhoppers. Additionally, the same broad resistance spectrum was observed in transgenic plants expressing $T y-1$, confirming the resistance is solely due to the presence of this resistance gene. Furthermore, this resistance is compromised by the presence of betasatellites, coreplicating DNA elements of begomoviruses from the Old World which encode $\beta C 1$, a suppressor of Transcriptional Gene Silencing (TGS). Further analysis has shown that in Ty-1 tomato the distribution profile of vsiRNAs are changed from those produced in susceptible Moneymaker (MM) tomato. The production of 21-nt vsiRNAs, the most dominant vsiRNAs in MM, is drastically reduced in Ty-1 tomato while both 22-nt and 24-nt vsiRNAs are increased. In addition, the distribution of vsiRNAs over the viral genome changes in Ty-1 plants: hotspots observed in MM plants are less pronounced in Ty-1 tomato, while the Intergenic Region and the open reading frame (ORF) V1 are relatively more targeted than in MM. Whereas cytoplasmic RNA processing bodies (PBs) compete for RNA substrates with siRNA bodies, where RDR6-based RNA interference (RNAi) amplification takes place, silencing of RNA decay elements from PBs leads to a stronger Post Transcriptional Gene Silencing (PTGS) response, but does not affect resistance against TYLCV. Cell biological studies has shown that in the absence of a viral infection Ty-1 localizes in the nucleus in proximity of COP1 bodies, while upon TYLCV infection Ty-1 condensates in a larger number of nuclear bodies that colocalize with cajal bodies. During this process Ty-1 protein interplays with elements from the sumoylation machinery, implying a role of sumoylation in the relocalization and/or functionalization of Ty-1.

Although the exact mode of action of Ty-1 still has not been solved and many questions remain, findings from this thesis research have implications for the practical use of Ty-1. In the following section, a few points will be highlighted and 
discussed in relation to practical and scientific implications with an outlook on possible future challenges.

\section{Resistance spectrum and disease management}

Ty-1 is used to combat TYLCV in tomato production. It was already established that Ty-1 provides resistance to other begomoviruses next to TYLCV (Barbieri et al., 2010; Butterbach et al., 2014; Pietersen and Smith, 2002; Prasanna et al., 2015; Shahid et al., 2013). However, the resistance turned out to extend beyond begomoviruses, as observed with the resistance against the curtovirus Beet curly top virus (BCTV) (Chapter 2). This is in agreement with our knowledge on RNAi, in which the RDR-mediated amplification is generic to provide a strong response either to all RNA viruses (PTGS) or to all DNA viruses (TGS). Considering Ty-1-mediated resistance is based on the amplification of RNAi, Ty-1 probably confers resistance to all geminiviruses, and not restricts to TYLCV and other Begomoviruses and Curtoviruses only. It is not unlikely that Ty-1 also confers resistance to other DNA viruses. Members of the Nanoviridae, multipartite viruses with a ssDNA genome, are suggested to be also prone to TGS (Pooggin, 2013; Thomas et al., 2021). It is tempting to speculate on the effect of Ty-1 on members of the Nanoviridae. Recently, Milk vetch dwarf virus (MDV), a member of the Nanovirus genus, members of which were thought to be restricted to Fabaceae plants, was identified from tomato, pepper (Solanaceae), lilly (Liliaceae) and papaya (Caricaceae) and a novel nanovirus was identified in parsley (Apiaceae) (Choi et al., 2019; Lal, Kil, et al., 2020; Lal, Vo, et al., 2020; Vetten et al., 2019; J., G., Yang et al., 2016). Because of their narrow host range, nanoviruses were always considered to have a limited impact. However, with the identification of MDV and the novel nanovirus in these other plants, their host range is clearly expanding beyond Fabaceae. This might turn nanoviruses into a growing threat for agriculture production systems and requires the need for resistance genes against nanoviruses. Whether Ty-1 presents one of those candidates will be interesting to test.

Since Ty-1 was the first TYLCV-resistance locus identified and inherited dominantly, many commercially available TYLCV-resistant tomato plants contain the Ty-1 
resistance gene (Gelbart et al., 2020; Zamir et al., 1994). However, both in a lab setting as under natural field conditions, Ty-1 resistance is compromised by coinfections with certain viruses or during co-replication of betasatellites (Butterbach et al., 2014; Conflon et al., 2018; Gelbart et al., 2020) (Chapter 2). TYLCV infections in tomato production systems can be quite devastating (Czosnek, 2007; Morales and Anderson, 2001). To maximize the efficacy of $T y-1$ and reduce yield losses in tomato production as much as possible, it is clear to not only surveil for the presence of TYLCV but also for other viruses and betasatellites that express TGS inhibitors and therefore compromise $T y$-1 resistance.

Next to co-infections also recombination events between TYLCV and TYLCSV have led to Ty-1 resistance breaking in the field. So far, the characterised resistance breaking strains are mostly similar to TYLCV and only a part in the intergenic region is derived from TYLCSV (Belabess et al., 2015; Belabess et al., 2016; Belabess et al., 2018; Torre et al., 2018; Urbino et al., 2020). Diagnostic tools have been developed to distinguish the parental TYLCV from the (Ty-1 breaking) recombinant virus, and it is recommended to screen Ty-1 bearing tomato cultivations for this TYLCV recombinant (Belabess et al., 2015). Recently, the fitness advantage of the resistance breaking strain was attributed to beneficial intra-genomic interactions. However, it was not further specified which intra-genomic interaction are playing a role (Urbino et al., 2020). Alignment of the genome of the Ty-1 resistance breaking strain (TYLCVIS76) along the 24-nt vsiRNA profile derived from Ty-1 plants (Chapter 3) showed an overlap of the recombination event with the genomic region that is more targeted by 24-nt visRNAs. This could suggest that TYLCV strains might evade from this targeting by recombination events within this genomic region. The observation that resistance breaking strain TYLCV-IS76 does have a slightly lower $\mathrm{G} / \mathrm{C}$ content in this region compared to the parental strain ( $44 \%$ and $48 \%$, respectively) is in agreement with this. On the other hand, several other resistant breaking strains do not have a low G/C content (Urbino et al., 2020). Additionally, no increased methylation was observed in the intergenic region of TYLCV in the presence of Ty-1 (Butterbach et al., 2014). The exact reason for a fitness advantage thus still remains unclear.

When the resistance mechanism of $T y-1$ is compared to other resistance strategies, certain pros and cons become evident. Ty-1 does not belong to the typical dominant 
NBS-LRR type of resistance genes, like the Ty-2 resistance gene against TYLCV. The latter triggers a resistance response, also visualized with a hypersensitive response, after recognition of the effector protein TYLCV C1 (Shen et al., 2020). Due to this selection pressure TYLCV may escape from, and break, Ty-2 resistance by changing the effector protein (de Ronde et al., 2014; Shen et al., 2020), which limits the durability of this resistance gene. Since $T y-1$ relies on RNAi, a generic defence mechanism against all RNA/DNA viruses that does not depend on the recognition of an effector protein, its resistance is probably more durable. However, a potential disadvantage of $T y-1$ is that the resistance is not absolute and the virus is still present in low amounts. From this "reservoir", viruses can still evolve and further spread via whitefly transmission to other susceptible plants (Marchant et al., 2020). How efficient the spread is from $T y-1$ bearing plants to susceptible plants and whether the presence of Ty-1 influences the feeding behaviour of whiteflies is not fully resolved (Lapidot et al., 2001; Legarrea et al., 2015; Srinivasan et al., 2012).

To increase resistance and durability, pyramiding of resistance genes is often considered (Dormatey et al., 2020; Mundt, 2018). By combining different resistance genes, pathogens have to overcome multiple resistance mechanisms to break resistance. In this context, combinations of $T y-2$ and $T y-3$ (an allele of $T y-1$ (Verlaan et al., 2013)), or Ty-1 and Ty-2, are used and shown to result in higher resistance level against several geminiviruses (Lee et al., 2021; Prasanna et al., 2015). Resistance from the wild tomato Solanum pimpinellifolium has also been combined with $T y-1$ and resulted in high resistance levels (Pérez De Castro et al., 2008). When the resistance mechanisms of the identified resistance genes are understood, it can be decided which genes to use in gene pyramiding to enhance the resistance levels and durability.

Another way to deploy RNAi-based resistance against TYLCV is via transgenic plants. Transformation and expression of transgene viral sequences (as an inverted repeat sequence), is giving rise to RNA molecules that fold into a dsRNA hairpin structure and become processed into siRNAs. As a result these plants contain antiviral RNA-Induced Silencing Complex (RISC) a priori and become totally immune to viral infection (Ammara et al., 2015; Leibman et al., 2015; Zrachya, Kumar, et al., 2007). The downside of this approach is that RNAi is highly sequence specific i.e., 
plants only become resistant to viruses that contain a sequence identical to that of the transgene. RNAi-mediated resistance thus leads to a very narrow resistance spectrum, but this can be overcome when viral sequences from different viruses are transformed simultaneously. Although engineered RNAi-mediated resistance is a very powerful tool to combat viral infections, not all ecological consequences can be predicted beforehand and partly due to this GMOs still receive societal reluctance (Vázquez-Barrios et al., 2021). As a result, the implementation of RDR genes comes most close to the (natural) exploitation and implementation of an enhanced RNAi response to combat and limit viral infections in agricultural food production systems. The biggest advantage from it is that it acts generic to all RNA viruses (by RDR-mediated PTGS), and all DNA viruses (by RDR-mediated TGS).

\section{The role of Ty-1 in RNAi pathways}

The antiviral RNAi response can be divided into the PTGS response and the TGS response (Mallory and Vaucheret, 2010). In the PTGS response, siRNAs of 21-22nt siRNAs are produced by Dicer-like protein (DCL)4/DCL2, which are subsequently loaded into a RISC complex containing Argonaute (AGO)1, to guide mRNA degradation or translational arrest of complementary sequences and this response is amplified by RDR6. Both AGO1 and RDR6 concentrate into cytoplasmic siRNA bodies while DCL4 is distributed throughout the cytoplasm (Mäkinen et al., 2017; Mallory and Vaucheret, 2010; Montavon et al., 2017; Pumplin and Voinnet, 2013). In the (canonical) TGS response, dsRNA is formed by the action of RDR2 and subsequently diced by DCL3 into 24-nt siRNAs. These are loaded into an AGO4 RISC complex, to guide methylation of complementary DNA and inhibit transcription (Erdmann and Picard, 2020; Pumplin and Voinnet, 2013). RDR2, DCL3 and AGO4 localize to the nuclear Cajal bodies (CBs) (Li et al., 2006; Pontes et al., 2006). One of the main questions of this thesis was to unravel in which of these pathways Ty-1 functions. A summary of the results from this thesis research is schematically depicted in Figure 6.1, and is further explained below.

So far, most evidence support a role of Ty-1 in the TGS pathway. Earlier, it was already reported that Ty-1 confers resistance to mono- and bipartite begomoviruses, 


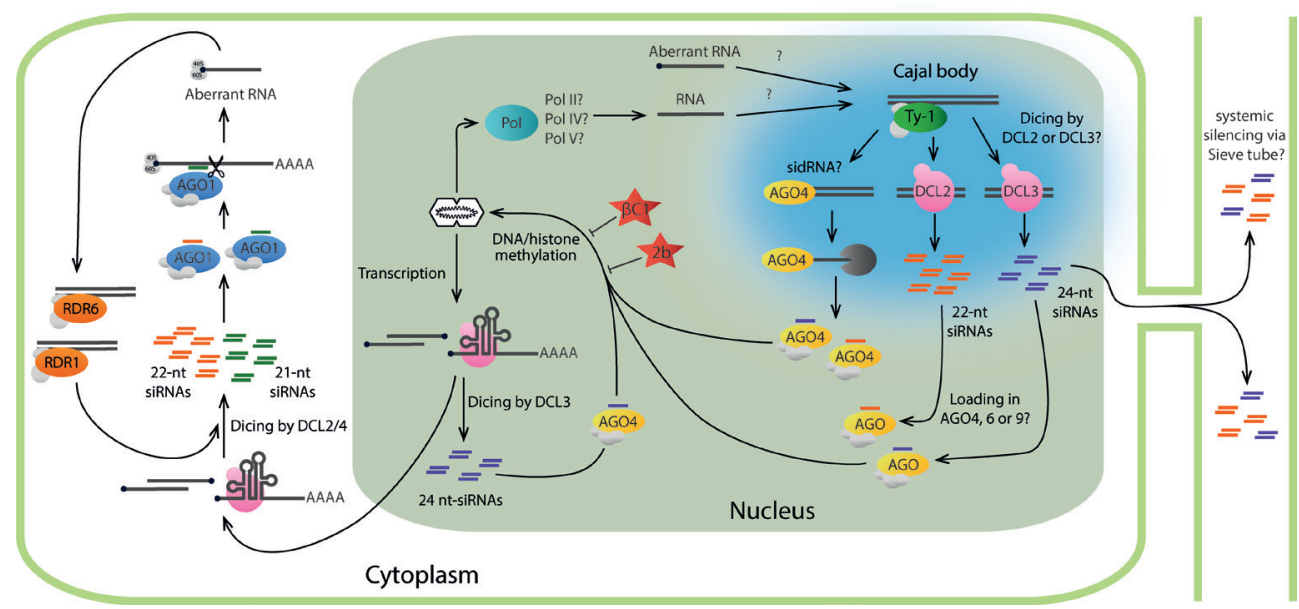

Figure 6.1: Model to position Ty-1 in the RNAi pathways.

Ty-1 is localized in the nuclear Cajal bodies during geminiviral infection, which also contain RNAi components AGO4, DCL3 and possibly DCL2. Ty-1 is proposed to convert geminiviral ssRNA (probably produced by Polymerase II, IV or V) into dsRNA, which next will be sliced into 22-nt and 24-nt siRNAs by the action of DCL3 and possibly nuclear-localized DCL2. Alternatively, processing by a 3'to 5'exonuclease resulting in dicer independent siRNAs (sidRNAs) might be occurring. 22-nt and 24-nt siRNAs will be loaded into AGO4, 6 or 9. Loaded AGO4, 6 or 9 are expected to find complementary viral DNA molecules and direct their methylation. Cucumber mosaic encoded protein $2 b$ and Tomato yellow leaf curl China betasatellite encoded $\beta C 1$ inhibit the DNA methylation. The formed 22-nt and 24-nt siRNAs can also be transported to sieve tubes to mediate systemic silencing.

but not to RNA viruses (Butterbach et al., 2014). Furthermore, geminiviral DNA collected from $T y-1$ bearing tomato showed increased levels of cytosine methylation (Butterbach et al., 2014). Data from this thesis has expanded the spectrum of resistance further towards the ssDNA viruses of the genera Curtovirus (Butterbach et al., 2014) (Chapter 2). A knock down of elements of the cytoplasmic P-bodies, which compete with RDR6-siRNA bodies for RNA substrate of the PTGS response, did not have an effect on TYLCV resistance. This indicates a spatial separation between the defence response against TYLCV and the interplaying P-bodies and siRNA bodies (Chapter 4). This was further supported by localization studies. Ty-1 localizes in nuclear bodies and during TYLCV infection, Ty-1 re-locates to nuclear CBs (Chapter 5). Lastly, in the presence of Ty-1, 24-nt viral siRNAs are increased compared to susceptible plants (Chapter 3 ). 
The localization of TGS components to CBs is a strong indication of its role in DNA methylation. However, most studies on TGS focus on silencing of transposable elements and transgenes. A recent article by Wang et. al. (2020), confirmed the importance of CBs in methylation of geminiviral DNA. TYLCV encoded V2 protein inhibits binding of AGO4 to the viral RNA and DNA in order to suppress DNA methylation and this interaction takes place in CBs. A variant V2 protein, which does not localize in CBs, does not suppress TGS. In addition, a knock down of CBS disturbs methylation of the geminiviral genome (Wang et al., 2020). The importance of CBs for geminiviral DNA methylation is in line with our observation that Ty-1 also localizes in CBs, making it likely that either the viral DNA is present in these nuclear structures at some point of the viral life cycle or (part of) the RNAi complexes needed for methylation are formed in or are passed through CBs.

An observation made in this thesis which does not seem in line with an enhanced TGS response, is the increase of 22-nt vsiRNAs in the presence of Ty-1. The 22-nt vsiRNAs are even the most prevalent in Ty-1 plants (Chapter 3). In general, 22-nt siRNAs are thought to be produced by DCL2 and to play a role in PTGS and antiviral defence against RNA viruses (Bouché et al., 2006; Garcia-Ruiz et al., 2010; Xie et al., 2004). However, to establish methylation, several non-canonical RdDM pathways use components of the PTGS machinery and 22-nt siRNAs (Cuerda-Gil and Slotkin, 2016; Erdmann and Picard, 2020; Kenchanmane Raju et al., 2019). In the RDR6-RdDM pathway, pol II mRNAs are converted in dsRNA by RDR6, which become cleaved by DCL2 or DCL4 in 21-22 nt siRNAs and load into AGO6, to guide the methylation of DNA (Cuerda-Gil and Slotkin, 2016; McCue et al., 2015; Nuthikattu et al., 2013). The Pol IV-NERD pathway is dependent on Pol IV and Pol V, but independent from RDR2 and AGO4. It involves the protein NERD (needed for RDR2-independent DNA methylation) and PTGS components RDR1/6, DCL2, DCL4, AGO2 and 21-nt and/ or 22-nt siRNAs. NERD binds to AGO2 and histones and this complex guides DNA methylation (Pontier et al., 2012). Another non-canonical RdDM pathway is the dicer independent siRNA (sidRNA) pathway. Longer RNA molecules produced by Pol II or PollV are bound by AGO4 and trimmed by a 3' to 5'exonuclease into a sidRNAs of 21-24 nt (Cuerda-Gil and Slotkin, 2016; D., L., Yang et al., 2016; Ye et al., 2016). Although speculative, whether the increased amounts of 22-nt viral siRNAs in Ty-1 
bearing tomato, relate to any of these non-canonical RdDM pathways remains an intriguing question to be answered.

The identity of the mobile RNAi signal, to initiate silencing in systemic plant tissue, is still under debate, but among others 22-nt and 24-nt siRNAs are suggested (W., Chen et al., 2018; Lewsey et al., 2016; Molnar et al., 2010; Taochy et al., 2017). In the phloem of plants siRNAs are found of 21-nt, 22-nt and 24-nt size classes (Buhtz et al., 2008; Yoo et al., 2004). Most studies unravelling functional mobile signals use knock out plants of several RNAi elements and combine this with grafting of scions, to determine factors necessary in the donor and acceptor tissues. The mobile signal inducing systemic TGS is thought to be mainly 24-nt, but also 22-nt siRNAs are mentioned (Lewsey et al., 2016; Melnyk et al., 2011; Molnar et al., 2010). One of the most interesting and unexpected observations from this study was the increased production level of 22-nt vsiRNAs, besides 24-nt vsiRNAs, in Ty-1 tomato lines. Since the virus is limited to the vascular system, in specific to phloem parenchymal and companion cells (Morilla et al., 2004) and is especially hampered by TGS, it is tempting to suggest that the increased amounts of both, 22- and 24-nt vsiRNAs are required to mount a TGS response to TYLCV.

High amounts of 22-nt viral siRNAs during geminivirus infection have been observed before and they were produced by DCL2 (Akbergenov et al., 2006; Yang, Wang, et al., 2011). DCL2 was long thought to be redundant in function for DCL4, but recently functions for DCL2 in antiviral defence, systemic silencing and transitivity are proposed (Parent et al., 2015; Taochy et al., 2017; T., Wang et al., 2018; Zhang et al., 2019). DCL2 stimulates secondary siRNA synthesis, resulting in transitivity (Mlotshwa et al., 2008; Parent et al., 2015). In the genomic distribution of siRNAs on the viral genome from Ty-1 plants hotspots were less clear and more equally spread out, most likely resulting from transitivity (Chapter 3). Although DCL2 primarily is known for its involvement in the production of 22-nt siRNAs involved in the cytoplasmic PTGS pathway, it also localizes (partly) in the nucleus (Pontes et al., 2013; Pumplin et al., 2016). From earlier studies it is also known that DCL2 knock out plants are hypersusceptible to the geminiviruses BCTV and Cabbage leaf curl virus (CaLCuV) (Raja et al., 2008). Altogether this makes it tempting to speculate on a mechanism in which Ty-1 helps to build a (nuclear) TGS response along two classes 
of vsiRNAs. A concerted action with DCL2 provides a 22-nt based TGS response, while in concert with DCL3 a 24-nt based response is generated (Figure 6.1).

\section{Functional regulation of Ty-1}

Defence responses in plants are tightly regulated to prevent loss of energy at the expense of plant growth (Huot et al., 2014). In addition, auto-immunity has to be prevented. For example, resistance genes of the NB-LRR kind should not be activated in the absence of a pathogen, because this can lead to a hypersensitive response (De Oliveira et al., 2016; de Ronde et al., 2014). RNAi can also result in auto-immunity: when RNA decay is inhibited, plant RNA transcripts enter the RNAi pathway resulting in inhibition of the production of essential proteins (De Alba et al., 2015; Gregory et al., 2008). Regulation takes place at the level of transcription but also via post translational modifications, like phosphorylation, ubiquitination and sumoylation (Buscaill and Rivas, 2014; Withers and Dong, 2017). The results presented in Chapter 5 show that the protein encoded by the resistance gene Ty-1 interacts with elements of the sumoylation machinery indicating a role for sumoylation in the regulation of its activity.

Sumoylation plays a major role in regulating plant defence (Verma et al., 2018). SUMO1/SUMO2 knock down plants and SIZ1 knock out plants are more resistant to Pseudomonas syringae, while knock down of SCE1 leads to higher susceptibility to Clavibacter michiganensis (van den Burg et al., 2010; Esparza-Araiza et al., 2015; Lee et al., 2006). In mammalian cells, viruses manipulate and interact with the sumoylation machinery (Everett et al., 2013; Lowrey et al., 2017; Wilson, 2017). However, limited data is available on the interplay of plant viruses and the sumoylation machinery. The only reported cases are for Turnip mosaic virus (TuMV) and the begomoviruses Tomato golden mosaic virus (TGMV), Tomato yellow leaf curl Sardinia virus (TYLCSV) and TYLCV (Cheng et al., 2017; Castillo et al., 2004; Cheng et al., 2017; Maio et al., 2019; Sanchez-Duran et al., 2011). The TuMV encoded RNA-dependent RNA polymerase (viral RdRP, named NIb) interacts with SCE1 and is sumoylated by SUMO3, which promotes viral infection (Cheng et al., 2017; Xiong and Wang, 2013). In addition, NIb sumoylation suppresses the host 
antiviral resistance pathway mediated by NPR1 (Cheng et al., 2017). Rep protein encoded by begomoviruses interact with SCE1, this interaction takes place in nuclear bodies (NBs) and suppresses the sumoylation of specific host proteins, including proliferating cell nuclear antigen (PCNA) (Arroyo-Mateos et al., 2018; Castillo et al., 2004; Maio et al., 2019; Sanchez-Duran et al., 2011). In yeast, impairment of PCNA sumoylation disrupts recruitment of the protein Srs2, resulting in higher levels of recombination events in replicating chromosomes (Pfander et al., 2005). So, Rep manipulates sumoylation to favour cellular conditions for viral replication (ArroyoMateos et al., 2018).

In the past few years, increasing evidence has been collected showing that TGS is also regulated by sumoylation. Studies unravelling the SUMOylome in plant cells, revealed many proteins involved in the canonical RNA dependent DNA methylation (RdDM) pathway being sumoylated (Augustine and Vierstra, 2018; J., Chen et al., 2018; Miller et al., 2010; Rytz et al., 2018). Amongst these proteins are methyltransferases MET1 and CHROMOMETHYLASE 3 (CMT3), and MICRORCHIDIA (MORC)-1, MORC-6, SU(VAR)3-9 HOMOLOG (SUVH)-2 which are part of protein complexes in the RdDM pathway (Augustine and Vierstra, 2018). In addition, direct effects of sumoylation on DNA methylation and TGS have been observed during different steps of the process. DNA methylation is a dynamic process and cytosine methylation can be removed by Repressor of silencing 1 (ROS1) (Zhang et al., 2018). ROS1 is stabilized by SIZ1-mediated sumoylation, so SIZ1 stimulates DNA demethylation (Kong et al., 2020). The protein SUVR2, which is a putative SU(VAR)39-like histone methyltransferase, is mono-sumoylated and sumoylation is required for the recruitment of chromatin remodelling proteins (Han et al., 2014; Luo et al., 2018). The DNA methyltransferase CMT3 interacts with SIZ1, becomes sumoylated and this enhances the DNA methyltransferase activity (Kim et al., 2015). In this respect, the observation on Ty-1 and its interaction with several components of the sumoylation machinery is not entirely unexpected, but reveals a modulation of the TGS response directly at the level of amplification.

To our knowledge, a role for sumoylation in the regulation of the core-components of RNAi in plants (DCL, AGO, RDR) has not been shown so far. In human alveolar macrophages, cigarette smoke may induce sumoylation of Dicer, resulting in 
decreased activity and lower miRNA production (Gross et al., 2014). In mammalian cells, sumoylation of Ago2 regulates its stability (Josa-Prado et al., 2015; Sahin et al., 2014). In this thesis, interactions between Ty-1, a RDRY, and SUMO-1, SUMO-3, SIZ1 and SCE1 were found and these interactions changed during TYLCV infection, suggesting a role for sumoylation in the regulation of the antiviral RNAi. Whether Ty-1 is sumoylated to change its stability or influences its conformation and interaction partners has to be analyzed, since sumoylation causes different effects depending on the targets (Flotho and Melchior, 2013).

The interactions between Ty-1 and COP1 remains more elusive. COP1 is an ubiquitin E3 ligase which regulates the photomorphogenic response, but also positively regulates HYL1, a protein required for miRNA processing, and the NB-LRR type resistance genes HRT1 and RPM1 (Cho et al., 2014; Jeong et al., 2010; Lau and Deng, 2012; Lim et al., 2018; Osterlund et al., 2000; Podolec and UIm, 2018; Saijo et al., 2003; Stacey et al., 1999; Yi and Deng, 2005). If and how COP1 regulates the resistance gene Ty-1, and whether light-induced modulation of defence responses play a role, will be challenging questions for the future.

\section{Ty-1 in cellular maintenance}

Although the focus of this thesis has been on the role of Ty-1 in antiviral TGS against geminiviruses, TGS is also required for cellular homeostasis by inhibiting transposable elements (TE) (Deniz et al., 2019; Erdmann and Picard, 2020). In a similar way, PTGS, involving the well-known RDR1 and -6 , is not only involved in an antiviral defence response but also involved into plant development processes as well. Along this line of thought, Ty-1 might, next to antiviral defence, have a function in other processes in the plant cell as well. To answer this question might become a big challenge. In the siRNA profiling increasing amount of 32-nt and 44-nt tomato specific siRNAs were observed in Ty-1 bearing plants (Chapter 3). Although these siRNAs are of yet unknown function, it would be of interest to identify their endogenous target RNA molecules. Another complicating factor relating to a putative role of Ty-1 in homeostasis, is the fact that susceptible MM plants don't have a functional copy of the $T y-1$ gene nor show any deficiency compared to Ty-1 bearing plants (Caro et 
al., 2015; Verlaan et al., 2013). Whether this is due to a possible redundancy with RDR2, involved in the canonical RdDM pathway and silencing of TE (Haag et al., 2012; Jia et al., 2009; Law et al., 2011), cannot be ruled out.

Table 6.1: Identified contigs/genes potentially coding for a RDR p protein.

\begin{tabular}{|c|c|c|c|c|}
\hline Crop & $\begin{array}{l}\text { Mono- } \\
\text { or di-cot }\end{array}$ & Genome & Hits & $\begin{array}{l}\text { Number } \\
\text { of } \\
\text { homologs }\end{array}$ \\
\hline Soybean & Dicot & $\begin{array}{l}\text { Glycine max Wm82.a2.v1 ge- } \\
\text { nome }\end{array}$ & >Glyma.01G008700.1 & 1 \\
\hline Pepper & Dicot & $\begin{array}{l}\text { capsicum annuum cv CM334 } \\
\text { Genome CDS release } 1.55\end{array}$ & $\begin{array}{l}>\text { CA08g03520 } \\
>\text { CA00g76620 }\end{array}$ & 2 \\
\hline Potato & Dicot & $\begin{array}{l}\text { Potato ITAG release } 1 \text { predicted } \\
\text { CDS (ST1.0) }\end{array}$ & $\begin{array}{l}\text { >Sotub06g014160.1.1 } \\
\text { >Sotub12g009540.1.1 }\end{array}$ & 2 \\
\hline Cassava & Dicot & Manihot esculenta v6.1 genome & - & 0 \\
\hline $\begin{array}{l}\text { Common } \\
\text { Bean }\end{array}$ & Dicot & Phaseolus vulgaris v2.1 genome & >Phvul.004G176400.1 & 1 \\
\hline Chickpea & Dicot & $\begin{array}{l}\text { Early release species: } \\
\text { Cicer arietinum v1.0 genome }\end{array}$ & $>$ Ca_17876 & 1 \\
\hline Cowpea & Dicot & $\begin{array}{l}\text { Early release species: } \\
\text { Vigna unguiculata v1.1 genome }\end{array}$ & >Vigun04g008500.1 & 1 \\
\hline Barley & Monocot & Hordeum vulgare $r 1$ genome & >HORVU3Hr1G027290.19 & 1 \\
\hline Maize & Monocot & Zea mays Ensembl-18 genome & >GRMZM2G327247_T01 & 1 \\
\hline Wheat & Monocot & $\begin{array}{l}\text { Early release species: } \\
\text { Triticum aestivum v2.2 genome }\end{array}$ & $\begin{array}{l}\text { >Traes_3AS_F27BB108C.2 } \\
>\text { Traes_7BL_8CEC8F99B.2 } \\
\text { > Traes_3DS_1C63C3CC4.1 } \\
>\text { Traes_3B_2C6DB84FB.2 } \\
\text { > Traes_3AS_FFA6E84DD.1 } \\
>\text { >Traes_3B_9CE9EAF60.1 }\end{array}$ & 3 \\
\hline Rice & Monocot & Oryza sativa v7_JGI genome & $\begin{array}{l}\text { >LOC_Os01g10140.2 } \\
>\text { >LOC_Os01g10130.2 }\end{array}$ & 2 \\
\hline
\end{tabular}

Results of a BLAST search with the coding sequence of Ty-1/RDR3/RDR4/RDR5 in phytozome.net or solgenomics.net (Fernandez-Pozo et al., 2015; Goodstein et al., 2012). For every found contig, it was checked whether it encodes the typical RDRy catalytic domain DFDGD and only the contigs that were positive are shown. After translating the contigs, the protein sequences were aligned to differentiate duplicates and homologs. 


\section{Future perspectives: RDRY proteins}

Ty-1 is a RDRy protein and the function of this class of proteins is so far unknown (Willmann et al., 2011). The role of Ty-1 in antiviral defence against geminiviruses, raises the question on the role of the other RDRy proteins in the biogenesis of vsiRNAs but also of endogenous host siRNAs. The model plant Arabidopsis thaliana encodes three RDR $\gamma$ proteins and also in $N$. benthamiana two RDR $\gamma$ proteins are expressed (Chapter 4, (Divi et al., 2014)). Another look into other economic important crops, and also affected by geminiviruses, like soybean, potato, pepper, barley and wheat, has revealed the presence of several potential RDR $\gamma$ homologs (Table 6.1, results of a BLAST search using the sequence of Ty-1 and RDR3, -4 and -5 with standard settings on either solgenomics.net or phytozome.net, only contigs that code for the typical RDR y catalytic domain DFDGD are shown). In all these crops the number of RDR $y$ copies differs, which not only makes it more puzzling but also raises an important question on their individual roles and possible redundancies. Being widespread in both monocots and dicots offers a challenge to investigate their potency as resistance gene against geminiviruses. This would not only increase the number of resistance traits that can be exploited for resistance breeding, but also provide more ways to generate durable resistance to a large variety of different viruses. A first glimpse of this may be collected from an observation made in soybean variety PK416, that exhibited a resistance against the bipartite geminivirus Mungbean yellow mosaic India virus (MYMIV), and showing only low MYMIV titers after a challenge with the virus (Ramesh et al., 2019). Furthermore, and interestingly, PK416 plants also revealed higher levels of 24-nt siRNAs specific for the intergenic region of DNA-A and a higher level of methylation of viral DNA compared to the susceptible variety (Yadav and Chattopadhyay, 2011). Although the resistance gene responsible has not been identified yet, this case shows high similarities to the situation of $T y-1$ bearing tomato lines. Considering soybean does contain a RDRY (Table 6.1) it is not unlikely that the observed MYMIV-resistance is due to a RDR $\gamma$ based resistance mechanism. 


\section{Concluding remarks}

The findings presented in this thesis give some further insight into the molecular mechanism behind Ty-1 mediated resistance against geminiviruses. So far, Ty-1 is the only identified resistance gene that enhances the antiviral TGS response and in this way confers a broad-spectrum resistance against geminiviruses. This makes it a very valuable gene for resistance breeding in tomato. Therefore, precautions have to be taken to ensure the durability of the resistance and prevent the emergence of resistance breaking strains, e.g. by pyramiding this gene with other types of resistance genes, but also by continuous screening for the composition of virus populations in outstanding crops (either resistance breaking strains or (RNA) viruses or satellites that might compromise its resistance) and optimise for disease management strategies.

The exact pathway and regulation of Ty- 1 , however, still is not very clear. First evidence has been collected that sumoylation plays a role in Ty-1-mediated resistance. Considering that the geminiviral protein Rep also interferes with sumoylation, although all consequences of this are not clear either (Arroyo-Mateos et al., 2018; Maio et al., 2019; Sanchez-Duran et al., 2011), indicates a very complex interplay between geminiviruses and their hosts, not only during the establishment of infections but also during host defence and virus counter defence. If and how geminiviral RNA silencing suppressors also act via sumoylation to tweak the regulation of these antiviral processes remains a challenging question. Unravelling such interactions may also help to unravel and understand fundamental cell biological processes involving TGS. Support for this may also come from studies on the known RDRy homologs in Arabidopsis and, if truly involving a similar component, the soybean variety PK416. Continuing efforts to further study and understand the action of this class of genes will tell us whether, or not, RDRy homologs become powerful new sources for future resistance breeding against a growing number of economically important, emerging viral DNA threats, and amongst which nowadays nanoviruses should be considered as well. 


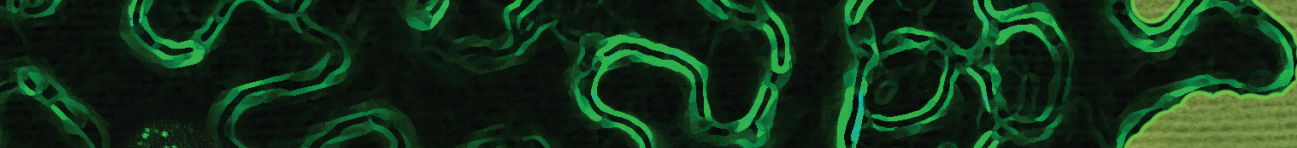

r.s. Wh 35)

2 2 ?

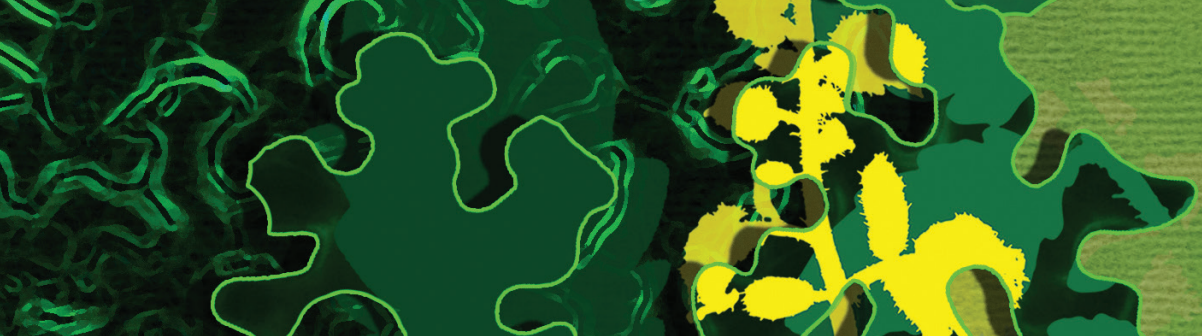

1 ?

) 20 203

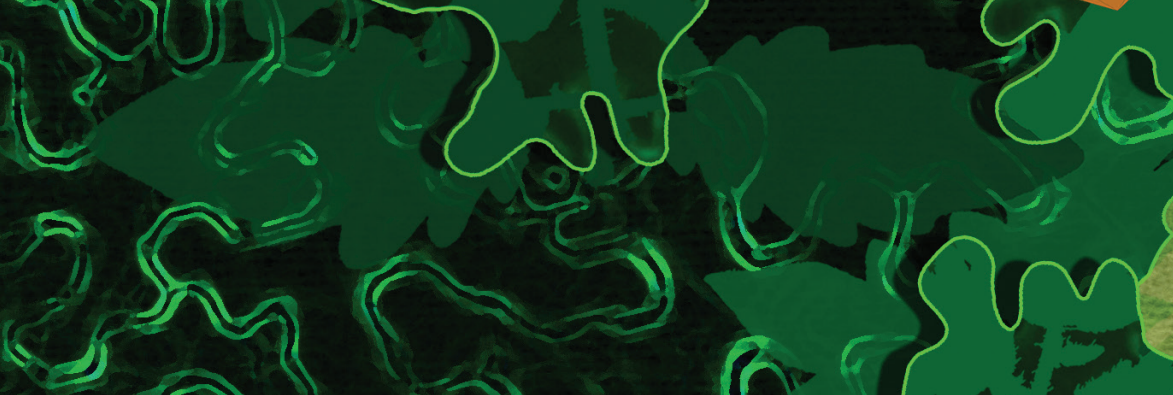




\section{Appendices}

References

Summary

Samenvatting

Acknowledgements

About the author

List of publications

Education statement 


\section{References}

Afgan, E., Baker, D., Batut, B., et al. (2018) The Galaxy platform for accessible, reproducible and collaborative biomedical analyses: 2018 update. Nucleic Acids Res. 46, W537-W544.

Akad, F., Eybishtz, A., Edelbaum, D., Gorovits, R., Dar-Issa, O., Iraki, N. and Czosnek, H. (2007) Making a friend from a foe: Expressing a GroEL gene from the whitefly Bemisia tabaci in the phloem of tomato plants confers resistance to tomato yellow leaf curl virus. Arch. Virol. 152, 1323-1339.

Akbergenov, R., Si-Ammour, A., Blevins, T., et al. (2006) Molecular characterization of geminivirus-derived small RNAs in different plant species. Nucleic Acids Res. 34, 462-471.

Alba, A.E.M. De, Moreno, A.B., Gabriel, M., et al. (2015) In plants, decapping prevents RDR6-dependent production of small interfering RNAs from endogenous mRNAs. Nucleic Acids Res. 43, 2902-2913.

Ali, Z., Abulfaraj, A., Idris, A., Ali, S., Tashkandi, M. and Mahfouz, M.M. (2015) CRISPR/Cas9-mediated viral interference in plants. Genome Biol. 16, 1-11.

Amin, I., Hussain, K., Akbergenov, R., Yadav, J.S., Qazi, J., Mansoor, S., Hohn, T., Fauquet, C.M. and Briddon, R.W. (2011) Suppressors of RNA silencing encoded by the components of the cotton leaf curl begomovirus-betasatellite complex. Mol. Plant-Microbe Interact. 24, 973-983.

Ammara, U.E., Mansoor, S., Saeed, M., Amin, I., Briddon, R.W. and Al-Sadi, A.M. (2015) RNA interferencebased resistance in transgenic tomato plants against Tomato yellow leaf curl virus-Oman (TYLCV-OM) and its associated betasatellite. Virol. J. 12, 1-12.

Appiano, M., Pavan, S., Catalano, D., Zheng, Z., Bracuto, V., Lotti, C., Visser, R.G.F., Ricciardi, L. and Bai, Y. (2015) Identification of candidate MLO powdery mildew susceptibility genes in cultivated Solanaceae and functional characterization of tobacco NtMLO1. Transgenic Res. 24, 847-858.

Aregger, M., Borah, B.K., Seguin, J., et al. (2012) Primary and Secondary siRNAs in Geminivirus-induced Gene Silencing. PLoS Pathog. 8, e1002941.

Arguello-Astorga, G., Lopez-Ochoa, L., Kong, L.J., Orozco, B.M., Settlage, S.B. and Hanley-Bowdoin, L. (2004) A novel motif in geminivirus replication proteins interacts with the plant retinoblastomarelated protein. J. Virol. 78, 4817-4826.

Arnim, A.G. von and Deng, X.-W. (1994) Light inactivation of arabidopsis photomorphogenic repressor COP1 involves a cell-specific regulation of its nucleocytoplasmic partitioning. Cell 79, 1035-1045.

Arroyo-Mateos, M., Sabarit, B., Maio, F., et al. (2018) Geminivirus Replication Protein impairs SUMO conjugation of PCNA at two acceptor sites. J. Virol. 92, e00611-18.

Augustine, R.C. and Vierstra, R.D. (2018) SUMOylation: re-wiring the plant nucleus during stress and development. Curr. Opin. Plant Biol. 45, 143-154.

Azevedo, J., Garcia, D., Pontier, D., et al. (2010) Argonaute quenching and global changes in Dicer homeostasis caused by a pathogen-encoded GW repeat protein. Genes Dev. 24, 904-915.

Barbieri, M., Acciarri, N., Sabatini, E., Sardo, L., Accotto, G.P. and Pecchioni, N. (2010) Introgression of resistance to two Mediterranean virus species causing Tomato yellow leaf curl into a valuable traditional tomato variety. J. Plant Pathol. 92, 485-493.

Baumberger, N., Tsai, C.H., Lie, M., Havecker, E. and Baulcombe, D.C. (2007) The Polerovirus Silencing Suppressor PO Targets ARGONAUTE Proteins for Degradation. Curr. Biol. 17, 1609-1614.

Belabess, Z., Dallot, S., El-Montaser, S., Granier, M., Majde, M., Tahiri, A., Blenzar, A., Urbino, C. and Peterschmitt, M. (2015) Monitoring the dynamics of emergence of a non-canonical recombinant of Tomato yellow leaf curl virus and displacement of its parental viruses in tomato. Virology 486, 291-306.

Belabess, Z., Peterschmitt, M., Granier, M., Tahiri, A., Blenzar, A. and Urbino, C. (2016) The non-canonical tomato yellow leaf curl virus recombinant that displaced its parental viruses in southern Morocco exhibits a high selective advantage in experimental conditions. J. Gen. Virol. 97, 3433-3445.

Belabess, Z., Urbino, C., Granier, M., Tahiri, A., Blenzar, A. and Peterschmitt, M. (2018) The typical RB76 recombination breakpoint of the invasive recombinant tomato yellow leaf curl virus of Morocco can be generated experimentally but is not positively selected in tomato. Virus Res. 243, 44-51.

Blevins, T., Podicheti, R., Mishra, V., Marasco, M., Wang, J., Rusch, D., Tang, H. and Pikaard, C.S. (2015) Identification of pol IV and RDR2-dependent precursors of 24 nt siRNAs guiding de novo DNA 
methylation in arabidopsis. Elife 4, e09591.

Blevins, T., Rajeswaran, R., Aregger, M., et al. (2011) Massive production of small RNAs from a non-coding region of Cauliflower mosaic virus in plant defense and viral counter-defense. Nucleic Acids Res. 39, 5003-5014.

Bologna, N.G. and Voinnet, O. (2014) The Diversity, Biogenesis, and Activities of Endogenous Silencing Small RNAs in Arabidopsis . Annu. Rev. Plant Biol. 65, 473-503.

Bond, D.M. and Baulcombe, D.C. (2015) Epigenetic transitions leading to heritable, RNA-mediated de novo silencing in Arabidopsis thaliana. Proc. Natl. Acad. Sci. 112, 917-922.

Borah, B.K., Zarreen, F., Baruah, G. and Dasgupta, I. (2016) Insights into the control of geminiviral promoters. Virology 495, 101-111.

Bouché, N., Lauressergues, D., Gasciolli, V. and Vaucheret, H. (2006) An antagonistic function for Arabidopsis DCL2 in development and a new function for DCL4 in generating viral siRNAs. EMBO J. 25, 3347-3356.

Brunetti, A., Tavazza, M., Noris, E., Tavazza, R., Caciagli, P., Ancora, G., Crespi, S. and Accotto, G.P. (1997) High expression of truncated viral rep protein confers resistance to tomato yellow leaf curl virus in transgenic tomato plants. Mol. Plant-Microbe Interact. 10, 571-579.

Bucher, E., Sijen, T., Haan, P. De, Goldbach, R. and Prins, M. (2003) Negative-strand tospoviruses and tenuiviruses carry a gene for a suppressor of gene silencing at analogous genomic positions. J. Virol. 77, 1329-1336.

Buchmann, R.C., Asad, S., Wolf, J.N., Mohannath, G. and Bisaro, D.M. (2009) Geminivirus AL2 and L2 Proteins Suppress Transcriptional Gene Silencing and Cause Genome-Wide Reductions in Cytosine Methylation. J. Virol. 83, 5005-5013.

Buhtz, A., Springer, F., Chappell, L., Baulcombe, D.C. and Kehr, J. (2008) Identification and characterization of small RNAs from the phloem of Brassica napus. Plant J. 53, 739-749.

Burg, H.A. van den, Kini, R.K., Schuurink, R.C. and Takken, F.L.W. (2010) Arabidopsis small ubiquitin-like modifier paralogs have distinct functions in development and defense. Plant Cell 22, 1998-2016.

Buscaill, P. and Rivas, S. (2014) Transcriptional control of plant defence responses. Curr. Opin. Plant Biol. 20, 35-46.

Buskirk, E.K. van, Decker, P. V. and Chen, M. (2012) Photobodies in light signaling. Plant Physiol. 158, 52-60.

Butterbach, P., Verlaan, M.G., Dullemans, A., Lohuis, D., Visser, R.G.F., Bai, Y. and Kormelink, R. (2014) Tomato yellow leaf curl virus resistance by Ty-1 involves increased cytosine methylation of viral genomes and is compromised by cucumber mosaic virus infection. Proc. Natl. Acad. Sci. 111, 1294212947.

Cao, M., Du, P., Wang, X., et al. (2014) Virus infection triggers widespread silencing of host genes by a distinct class of endogenous siRNAs in Arabidopsis. Proc. Natl. Acad. Sci. 111, 14613-14618.

Carluccio, A.V., Prigigallo, M.I., Rosas-Diaz, T., Lozano-Duran, R. and Stavolone, L. (2018) S-acylation mediates Mungbean yellow mosaic virus AC4 localization to the plasma membrane and in turns gene silencing suppression. PLoS Pathog. 14, 1-26.

Caro, M., Verlaan, M.G., Julián, O., et al. (2015) Assessing the genetic variation of Ty-1 and Ty-3 alleles conferring resistance to tomato yellow leaf curl virus in a broad tomato germplasm. Mol. Breed. 35, $1-13$.

Castillo-González, C., Liu, X., Huang, C., et al. (2015) Geminivirus-encoded TrAP suppressor inhibits the histone methyltransferase SUVH4/KYP to counter host defense. Elife 4, e06671.

Castillo, A.G., Collinet, D., Deret, S., Kashoggi, A. and Bejarano, E.R. (2003) Dual interaction of plant PCNA with geminivirus replication accessory protein (REn) and viral replication protein (Rep). Virology 312, 381-394.

Castillo, A.G., Kong, L.J., Hanley-Bowdoin, L. and Bejarano, E.R. (2004) Interaction between a Geminivirus Replication Protein and the Plant Sumoylation System. J. Virol. 78, 2758-2769.

Castro, A.P. De, Díez, M.J. and Nuez, F. (2005) Evaluation of breeding tomato lines partially resistant to Tomato yellow leaf curl Sardinia virus and Tomato yellow leaf curl virus derived from Lycopersicon chilense. Can. J. Plant Pathol. 27, 268-275.

Ceniceros-Ojeda, E.A., Rodríguez-Negrete, E.A. and Rivera-Bustamante, R.F. (2016) Two Populations of Viral Minichromosomes Are Present in a Geminivirus-Infected Plant Showing Symptom Remission (Recovery). J. Virol. 90, 3828-3838. 
Chellappan, P., Vanitharani, R. and Fauquet, C.M. (2005) MicroRNA-binding viral protein interferes with Arabidopsis development. Proc. Natl. Acad. Sci. 102, 10381-10386.

Chen, J., Kalinowska, K., Müller, B., Mergner, J., Deutzmann, R., Schwechheimer, C., Hammes, U.Z. and Dresselhaus, T. (2018) DiSUMO-LIKE Interacts with RNA-Binding Proteins and Affects Cell-Cycle Progression during Maize Embryogenesis. Curr. Biol. 28, 1548-1560.

Chen, W., Zhang, X., Fan, Y., et al. (2018) A Genetic Network for Systemic RNA Silencing in Plants. Plant Physiol. 176, 2700-2719.

Cheng, X., Xiong, R., Li, Y., Li, F., Zhou, X. and Wang, A. (2017) Sumoylation of Turnip mosaic virus RNA Polymerase Promotes Viral Infection by Counteracting the Host NPR1-Mediated Immune Response. Plant Cell 29, 508-525.

Cho, S.K., Chaabane, S. Ben, Shah, P., Poulsen, C.P. and Yang, S.W. (2014) COP1 E3 ligase protects HYL1 to retain microRNA biogenesis. Nat. Commun. 5, 5867.

Choi, H., Jo, Y., Zhou, Y. and Cho, W.K. (2019) First Report of Milk Vetch Dwarf Virus Infecting Lily in Korea. Plant Dis. 103, 2144.

Chowda-Reddy, R. V., Achenjang, F., Felton, C., Etarock, M.T., Anangfac, M.T., Nugent, P. and Fondong, V.N. (2008) Role of a geminivirus AV2 protein putative protein kinase $C$ motif on subcellular localization and pathogenicity. Virus Res. 135, 115-124.

Cohen, S. and Harpaz, I. (1964) Periodic, rather than continual acquisition of a new tomato virus by its vector, the tobacco whitefly (Bemisia tabaci Gennadius). Entomol. Exp. Appl. 7, 155-166.

Combe, J.P., Petracek, M.E., Eldik, G. Van, Meulewaeter, F. and Twell, D. (2005) Translation initiation factors elF4E and elFiso4E are required for polysome formation and regulate plant growth in tobacco. Plant Mol. Biol. 57, 749-760.

Conflon, D., Granier, M., Tiendrébéogo, F., Gentit, P., Peterschmitt, M. and Urbino, C. (2018) Accumulation and transmission of alphasatellite, betasatellite and tomato yellow leaf curl virus in susceptible and Ty-1-resistant tomato plants. Virus Res. 253, 124-134.

Conti, G., Zavallo, D., Venturuzzi, A.L., Rodriguez, M.C., Crespi, M. and Asurmendi, S. (2017) TMV induces RNA decay pathways to modulate gene silencing and disease symptoms. Plant J. 89, 73-84.

Coursey, T., Regedanz, E. and Bisaro, D.M. (2018) Arabidopsis RNA Polymerase V Mediates Enhanced Compaction and Silencing of Geminivirus and Transposon Chromatin During Host Recovery from Infection. J. Virol. 92, e01320-17.

Csorba, T., Kontra, L. and Burgyán, J. (2015) viral silencing suppressors: Tools forged to fine-tune hostpathogen coexistence. Virology 479-480, 85-103.

Csorba, T., Lózsa, R., Hutvágner, G. and Burgyán, J. (2010) Polerovirus protein P0 prevents the assembly of small RNA-containing RISC complexes and leads to degradation of ARGONAUTE1. Plant J. 62, 463472.

Cuerda-Gil, D. and Slotkin, R.K. (2016) Non-canonical RNA-directed DNA methylation. Nat. Plants 2, 16163.

Cui, X., Li, G., Wang, D., Hu, D. and Zhou, X. (2005) A Begomovirus DNAbeta-Encoded Protein Binds DNA, Functions as a Suppressor of RNA Silencing, and Targets the Cell Nucleus. J. Virol. 79, 10764-10775.

Cui, X. and Zhou, X. (2004) AC2 and AC4 proteins of Tomato yellow leaf curl China virus and Tobacco curly shoot virus mediate suppression of RNA silencing. Chinese Sci. Bull. 49, 2607-2612.

Cuperus, J.T., Carbonell, A., Fahlgren, N., et al. (2010) Unique functionality of 22-nt miRNAs in triggering RDR6-dependent siRNA biogenesis from target transcripts in Arabidopsis. Nat. Struct. Mol. Biol. 17, 997-1003.

Czosnek, H. (2007) Tomato Yellow Leaf Curl Virus Disease: Management, Molecular Biology, Breeding for Resistance, Dordrecht, Springer Netherlands.

Czosnek, H., Hariton-Shalev, A., Sobol, I., Gorovits, R. and Ghanim, M. (2017) The incredible journey of Begomoviruses in their whitefly vector. Viruses $\mathbf{9}, 273$.

Deniz, Ö., Frost, J.M. and Branco, M.R. (2019) Regulation of transposable elements by DNA modifications. Nat. Rev. Genet. 20, 417-431.

Ding, S.-W. and Voinnet, O. (2007) Antiviral Immunity Directed by Small RNAs. Cell 130, 413-426.

Ding, Y. and Lozano-Durán, R. (2020) The cajal body in plant-virus interactions. Viruses 12, 250.

Divi, U., Tahchy, A. El, Vanhercke, T., Petrie, J. and Singh, S. (2013) Nicotiana benthamiana leaf transcriptome. CSIRO data Collect. v1. 
Divi, U.K., Tahchy, A. El, Vanhercke, T., Petrie, J.R., Robles-Martinez, J.A. and Singh, S.P. (2014) Transcriptional and biochemical responses of monoacylglycerol acyltransferase-mediated oil synthesis and associated senescence-like responses in Nicotiana benthamiana. Front. Plant Sci. 5, 204.

Dogra, S.C., Eini, O., Rezaian, M.A. and Randles, J.W. (2009) A novel shaggy-like kinase interacts with the tomato leaf curl virus pathogenicity determinant C4 protein. Plant Mol. Biol. 71, 25-38.

Donaire, L., Barajas, D., Martínez-García, B., Martínez-Priego, L., Pagán, I. and Llave, C. (2008) Structural and Genetic Requirements for the Biogenesis of Tobacco Rattle Virus-Derived Small Interfering RNAs. J. Virol. 82, 5167-5177.

Dong, X., Wezel, R. van, Stanley, J. and Hong, Y. (2003) Functional Characterization of the Nuclear Localization Signal for a Suppressor of Posttranscriptional Gene Silencing. J. Virol. 77, 7026-7033.

Dormatey, R., Sun, C., Ali, K., Coulter, J.A., Bi, Z. and Bai, J. (2020) Gene pyramiding for sustainable crop improvement against biotic and abiotic stresses. Agronomy 10, 1255.

Doyle, J.J. and Doyle, J.L. (1987) A rapid DNA isolation procedure for small quantities of fresh leaf tissue. Phytochem. Bull. 19, 11-15.

Duan, C., Fang, Y., Zhou, B., Zhao, J., Hou, W., Zhu, H., Ding, S. and Guo, H. (2012) Suppression of Arabidopsis ARGONAUTE1-Mediated Slicing, Transgene-Induced RNA Silencing, and DNA Methylation by Distinct Domains of the Cucumber mosaic virus 2b Protein. Plant Cell 24, 259-274.

Duan, C., Zhang, H., Tang, K., et al. (2015) Specific but interdependent functions for Arabidopsis AGO 4 and AGO 6 in RNA-directed DNA methylation. EMBO J. 34, 581-592.

Eini, O. (2017) A betasatellite-encoded protein regulates key components of gene silencing system in plants. Mol. Biol. 51, 579-585.

Eini, O., Dogra, S.C., Dry, I.B. and Randles, J.W. (2012) Silencing suppressor activity of a begomovirus DNA $\beta$ encoded protein and its effect on heterologous helper virus replication. Virus Res. 167, 97-101.

Erdmann, R.M. and Picard, C.L. (2020) RNA-directed DNA Methylation. PLoS Genet. 16, e1009034.

Esparza-Araiza, M.J., Bañuelos-Hernández, B., Argüello-Astorga, G.R., Lara-Ávila, J.P., Goodwin, P.H., Isordia-Jasso, M.I., Castillo-Collazo, R., Rougon-Cardoso, A. and Alpuche-Solís, Á.G. (2015) Evaluation of a SUMO E2 conjugating enzyme involved in resistance to clavibacter michiganensis subsp. Michiganensis in solanum peruvianum, through a tomato mottle virus VIGS assay. Front. Plant Sci. 6, 1019.

Everett, R.D., Boutell, C. and Hale, B.G. (2013) Interplay between viruses and host sumoylation pathways. Nat. Rev. Microbiol. 11, 400-411.

Felippes, F.F. de and Waterhouse, P.M. (2020) The Whys and Wherefores of Transitivity in Plants. Front. Plant Sci. 11, 579376.

Fernandez-Pozo, N., Menda, N., Edwards, J.D., et al. (2015) The Sol Genomics Network (SGN)-from genotype to phenotype to breeding. Nucleic Acids Res. 43, D1036-D1041.

Flotho, A. and Melchior, F. (2013) Sumoylation: A regulatory protein modification in health and disease. Annu. Rev. Biochem. 82, 357-385.

Fondong, V.N., Reddy, R.V.C., Lu, C., Hankoua, B., Felton, C., Czymmek, K. and Achenjang, F. (2007) The consensus $\mathrm{N}$-myristoylation motif of a geminivirus AC4 protein is required for membrane binding and pathogenicity. Mol. Plant-Microbe Interact. 20, 380-391.

Friedmann, M., Lapidot, M., Cohen, S. and Pilowsky, M. (1998) A Novel Source of Resistance to Tomato Yellow Leaf Curl Virus Exhibiting a Symptomless Reaction to Viral Infection. J. Am. Soc. Hortic. Sci. 123, 1004-1007.

Fukunaga, R. and Doudna, J.A. (2009) dsRNA with 5' overhangs contributes to endogenous and antiviral RNA silencing pathways in plants. EMBO J. 28, 545-555.

Fulton, T.M., Chunwongse, J. and Tanksley, S.D. (1995) Microprep protocol for extraction of DNA from tomato and other herbaceous plants. Plant Mol. Biol. Report. 13, 207-209.

Furuta, K.M., Yadav, S.R., Lehesranta, S., et al. (2014) Arabidopsis NAC45/86 direct sieve element morphogenesis culminating in enucleation. Science 345, 933-937.

Gallois, J., Charron, C., Sánchez, F., et al. (2010) Single amino acid changes in the turnip mosaic virus viral genome-linked protein (VPg) confer virulence towards Arabidopsis thaliana mutants knocked out for eukaryotic initiation factors elF(iso)4E and elF(iso)4G. J. Gen. Virol. 91, 288-293.

Garcia-Ruiz, H., Takeda, A., Chapman, E.J., Sullivan, C.M., Fahlgren, N., Brempelis, K.J. and Carrington, J.C. 
(2010) Arabidopsis RNA-dependent RNA polymerases and dicer-like proteins in antiviral defense and small interfering RNA biogenesis during Turnip mosaic virus infection. Plant Cell 22, 481-496.

Gazzani, S., Lawrenson, T., Woodward, C., Headon, D. and Sablowski, R. (2004) A link between mRNA turnover and RNA interference in Arabidopsis. Science 306, 1046-1048.

Gelbart, D., Chen, L., Alon, T., Dobrinin, S., Levin, I. and Lapidot, M. (2020) The recent association of a DNA betasatellite with Tomato yellow leaf curl virus in Israel - A new threat to tomato production. Crop Prot. 128, 104995.

Gill, U.S., Lee, S. and Mysore, K.S. (2015) Host Versus Nonhost Resistance: Distinct Wars with Similar Arsenals. Phytopathology 105, 580-587.

Glick, E., Levy, Y., Gafni, Y., Dagan, B. and Mina, T. (2009) The Viral Etiology of Tomato Yellow Leaf Curl Disease - A Review. Plant Prot. Sci. 45, 81-97.

Glick, E., Zrachya, A., Levy, Y., Mett, A., Gidoni, D., Belausov, E., Citovsky, V. and Gafni, Y. (2008) Interaction with host SGS3 is required for suppression of RNA silencing by tomato yellow leaf curl virus V2 protein. Proc. Natl. Acad. Sci. 105, 157-161.

González, I., Martínez, L., Rakitina, D. V, et al. (2010) Cucumber Mosaic Virus 2b Protein Subcellular Targets and Interactions: Their Significance to RNA Silencing Suppressor Activity. Mol. Plant-Microbe Interact. 23, 294-303.

Goodstein, D.M., Shu, S., Howson, R., et al. (2012) Phytozome: A comparative platform for green plant genomics. Nucleic Acids Res. 40, D1178-D1186.

Gopal, P., Pravin Kumar, P., Sinilal, B., Jose, J., Kasin Yadunandam, A. and Usha, R. (2007) Differential roles of $C 4$ and $\beta C 1$ in mediating suppression of post-transcriptional gene silencing: Evidence for transactivation by the $\mathrm{C} 2$ of Bhendi yellow vein mosaic virus, a monopartite begomovirus. Virus Res. 123, 9-18.

Gou, M., Huang, Q., Qian, W., Zhang, Z., Jia, Z. and Hua, J. (2017) Sumoylation E3 Ligase SIZ1 Modulates Plant Immunity Partly through the Immune Receptor Gene SNC1 in Arabidopsis. Mol. Plant-Microbe Interact. 30, 334-342.

Granier, M., Tomassoli, L., Manglli, A., Nannini, M., Peterschmitt, M. and Urbino, C. (2019) First report of TYLCV-IS141, a Tomato yellow leaf curl virus recombinant infecting tomato plants carrying the Ty-1 resistance gene in Sardinia (Italy). Plant Dis. 103, 1-4.

Gregory, B.D., O'Malley, R.C., Lister, R., Urich, M.A., Tonti-Filippini, J., Chen, H., Millar, A.H. and Ecker, J.R. (2008) A Link between RNA Metabolism and Silencing Affecting Arabidopsis Development. Dev. Cell 14, 854-866.

Gross, T.J., Powers, L.S., Boudreau, R.L., Brink, B., Reisetter, A., Goel, K., Gerke, A.K., Hassan, I.H. and Monick, M.M. (2014) A Microrna processing defect in smokers' macrophages is linked to sumoylation of the endonuclease DICER. J. Biol. Chem. 289, 12823-12834.

Gutierrez, C. (2000) Geminiviruses and the plant cell cycle. Plant Mol. Biol. 43, 763-772.

Gy, I., Gasciolli, V., Lauressergues, D., Morel, J.-B., Gombert, J., Proux, F., Proux, C., Vaucheret, H. and Mallory, A.C. (2007) Arabidopsis FIERY1, XRN2, and XRN3 Are Endogenous RNA Silencing Suppressors. Plant Cell 19, 3451-3461.

Haag, J.R., Ream, T.S., Marasco, M., Nicora, C.D., Norbeck, A.D., Pasa-Tolic, L. and Pikaard, C.S. (2012) In Vitro Transcription Activities of Pol IV, Pol V, and RDR2 Reveal Coupling of Pol IV and RDR2 for dsRNA Synthesis in Plant RNA Silencing. Mol. Cell 48, 811-818.

Hak, H., Levy, Y., Chandran, S.A., Belausov, E., Loyter, A., Lapidot, M. and Gafni, Y. (2015) TYLCV-IS movement in planta does not require V2 protein. Virology 477, 56-60.

Hamera, S., Song, X., Su, L., Chen, X. and Fang, R. (2012) Cucumber mosaic virus suppressor 2b binds to AGO4-related small RNAs and impairs AGO4 activities. Plant J. 69, 104-115.

Hammond, S.M. (2005) Dicing and slicing: The core machinery of the RNA interference pathway. FEBS Lett. 579, 5822-5829.

Han, M.H., Goud, S., Song, L. and Fedoroff, N. (2004) The Arabidopsis double-stranded RNA-binding protein HYL1 plays a role in microRNA-mediated gene regulation. Proc. Natl. Acad. Sci. 101, 1093-1098.

Han, Y.F., Dou, K., Ma, Z.Y., et al. (2014) SUVR2 is involved in transcriptional gene silencing by associating with SNF2-related chromatin-remodeling proteins in Arabidopsis. Cell Res. 24, 1445-1465.

Hanson, P., Green, S.K. and Kuo, G. (2006) Ty-2, a gene on chromosome 11 conditioning geminivirus 
resistance in tomato. Tomato Genet. Coop. Rep. 56, 17-18.

Hao, L., Wang, H., Sunter, G. and Bisaro, D.M. (2003) Geminivirus AL2 and L2 Proteins Interact with and Inactivate SNF1 Kinase. Plant Cell 15, 1034-1048.

Havecker, E.R., Wallbridge, L.M., Hardcastle, T.J., Bush, M.S., Kelly, K.A., Dunn, R.M., Schwach, F., Doonan, J.H. and Baulcombe, D.C. (2010) The arabidopsis RNA-directed DNA methylation argonautes functionally diverge based on their expression and interaction with target loci. Plant Cell 22, 321-334.

Hayashi, M., Nanba, C., Saito, M., Kondo, M., Takeda, A., Watanabe, Y. and Nishimura, M. (2012) Loss of XRN4 function can trigger cosuppression in a sequence-dependent manner. Plant Cell Physiol. 53, 1310-1321.

Hecker, C.-M., Rabiller, M., Haglund, K., Bayer, P. and Dikic, I. (2006) Specification of SUMO1- and SUMO2interacting motifs. J. Biol. Chem. 281, 16117-16127.

Hedil, M. and Kormelink, R. (2016) Viral RNA Silencing Suppression : The Enigma of Bunyavirus NSs Proteins. Viruses 8, 208.

Hedil, M., Ronde, D. de and Kormelink, R. (2017) Biochemical analysis of NSs from different tospoviruses. Virus Res. 242, 149-155.

Hedil, M., Sterken, M.G., Ronde, D. De, Lohuis, D. and Kormelink, R. (2015) Analysis of tospovirus NSs proteins in suppression of systemic silencing. PLoS One 10, e0134517.

Heilersig, H.J.B., Loonen, A., Bergervoet, M., Wolters, A.M.A. and Visser, R.G.F. (2006) Post-transcriptional gene silencing of GBSSI in potato: Effects of size and sequence of the inverted repeats. Plant Mol. Biol. 60, 647-662.

Hématy, K., Bellec, Y., Podicheti, R., et al. (2016) The Zinc-Finger Protein SOP1 Is Required for a Subset of the Nuclear Exosome Functions in Arabidopsis. PLoS Genet. 12, e1005817.

Hendriks, I.A., D'Souza, R.C., Chang, J.G., Mann, M. and Vertegaal, A.C.O. (2015) System-wide identification of wild-type SUMO-2 conjugation sites. Nat. Commun. 6, 7289.

Höck, J. and Meister, G. (2008) The Argonaute protein family. Genome Biol. 9, 210.

Hou, Y., Zhai, Y., Feng, L., et al. (2019) A Phytophthora Effector Suppresses Trans-Kingdom RNAi to Promote Disease Susceptibility. Cell Host Microbe 25, 153-165.

Hsieh, Y.-C., Omarov, R.T. and Scholthof, H.B. (2009) Diverse and Newly Recognized Effects Associated with Short Interfering RNA Binding Site Modifications on the Tomato Bushy Stunt Virus P19 Silencing Suppressor. J. Virol. 83, 2188-2200.

Huot, B., Yao, J., Montgomery, B.L. and He, S.Y. (2014) Growth-defense tradeoffs in plants: A balancing act to optimize fitness. Mol. Plant 7, 1267-1287.

Hutton, S.F. and Scott, J.W. (2014) Ty-6, a major begomovirus resistance gene located on chromosome 10. Rep. tomato Genet. Coop. 64, 14-18.

Hutton, S.F., Scott, J.W. and Schuster, D.J. (2012) Recessive resistance to tomato yellow leaf curl virus from the tomato cultivar Tyking is located in the same region as Ty-5 on chromosome 4. HortScience 47, 324-327.

Ismayil, A., Haxim, Y., Wang, Y., et al. (2018) Cotton Leaf Curl Multan virus C4 protein suppresses both transcriptional and post-transcriptional gene silencing by interacting with SAM synthetase. PLoS Pathog. 14, e1007282.

Jackel, J.N., Buchmann, R.C., Singhal, U. and Bisaro, D.M. (2015) Analysis of Geminivirus AL2 and L2 Proteins Reveals a Novel AL2 Silencing Suppressor Activity. J. Virol. 89, 3176-3187.

Jackel, J.N., Storer, J.M., Coursey, T. and Bisaro, D.M. (2016) Arabidopsis RNA Polymerases IV and V Are Required To Establish H3K9 Methylation, but Not Cytosine Methylation, on Geminivirus Chromatin. J. Virol. 90, 7529-7540.

Jang, I.-C., Yang, J.-Y., Seo, H.S. and Chua, N.-H. (2005) HFR1 is targeted by COP1 E3 ligase for posttranslational proteolysis during phytochrome A signaling. Genes Dev. 19, 593-602.

Jashni, M.K., Dols, I.H.M., lida, Y., Boeren, S., Beenen, H.G., Mehrabi, R., Collemare, J. and Wit, P.J.G.M. de (2015) Synergistic Action of a Metalloprotease and a Serine Protease from Fusarium oxysporum f. sp. lycopersici Cleaves Chitin-Binding Tomato Chitinases, Reduces Their Antifungal Activity, and Enhances Fungal Virulence. Mol. Plant Microbes Interact. 28, 996-1008.

Jeong, R.-D., Chandra-Shekara, A.C., Barman, S.R., Navarre, D., Klessig, D.F., Kachroo, A. and Kachroo, P. (2010) Cryptochrome 2 and phototropin 2 regulate resistance protein-mediated viral defense by 
negatively regulating an E3 ubiquitin ligase. Proc. Natl. Acad. Sci. 107, 13538-13543.

Jeske, H., Lütgemeier, M. and Preiß, W. (2001) DNA forms indicate rolling circle and recombinationdependent replication of Abutilon mosaic virus. EMBO J. 20, 6158-6167.

Ji, Y., Schuster, D.J. and Scott, J.W. (2007) Ty-3, a begomovirus resistance locus near the Tomato yellow leaf curl virus resistance locus Ty-1 on chromosome 6 of tomato. Mol. Breed. 20, 271-284.

Ji, Y., Scott, J.W. and Schuster, D.J. (2009) Molecular Mapping of Ty-4 , a New Tomato Yellow Leaf Curl Virus Resistance Locus on Chromosome 3 of Tomato. J. Am. Soc. Hortic. Sci. 134, 281-288.

Jia, Y., Lisch, D.R., Ohtsu, K., Scanlon, M.J., Nettleton, D. and Schnable, P.S. (2009) Loss of RNA-dependent RNA polymerase 2 (RDR2) function causes widespread and unexpected changes in the expression of transposons, genes, and 24-nt small RNAs. PLoS Genet. 5, e1000737.

Jiang, L., Qian, D., Zheng, H., et al. (2012) RNA-dependent RNA polymerase 6 of rice (Oryza sativa) plays role in host defense against negative-strand RNA virus, Rice stripe virus. Virus Res. 163, 512-519.

Jones, L., Hamilton, A.J., Voinnet, O., Thomas, C.L., Maule, A.J. and Baulcombe, D.C. (1999) RNA-DNA Interactions and DNA Methylation in Post-Transcriptional Gene Silencing. Plant Cell 11, 2291-2301.

Josa-Prado, F., Henley, J.M. and Wilkinson, K.A. (2015) SUMOylation of Argonaute-2 regulates RNA interference activity. Biochem. Biophys. Res. Commun. 464, 1066-1071.

Jupin, I., Kouchkovsky, F. De, Jouanneau, F. and Gronenborn, B. (1994) Movement of tomato yellow leaf curl geminivirus (TYLCV): Involvement of the protein encoded by ORF C4. Virology 204, 82-90.

Kapos, P., Devendrakumar, K.T. and Li, X. (2019) Plant NLRs: From discovery to application. Plant Sci. 279, 3-18.

Kawakatsu, T. and Ecker, J.R. (2019) Diversity and dynamics of dna methylation: Epigenomic resources and tools for crop breeding. Breed. Sci. 69, 191-204.

Kelly, L., Mezulis, S., Yates, C., Wass, M. and Sternberg, M. (2015) The Phyre2 web portal for protein modeling, prediction and analysis. Nat. Protoc. 10, 845-858.

Kenchanmane Raju, S.K., Ritter, E.J. and Niederhuth, C.E. (2019) Establishment, maintenance, and biological roles of non-CG methylation in plants. Essays Biochem. 63, 743-755.

Kerscher, O. (2007) SUMO junction-what's your function? New insights through SUMO-interacting motifs. EMBO Rep. 8, 550-555.

Kim, D.Y., Han, Y.J., Kim, S. Il, Song, J.T. and Seo, H.S. (2015) Arabidopsis CMT3 activity is positively regulated by AtSIZ1-mediated sumoylation. Plant Sci. 239, 209-215.

Kim, S.H., Ryabov, E. V., Kalinina, N.O., Rakitina, D. V., Gillespie, T., MacFarlane, S., Haupt, S., Brown, J.W.S. and Taliansky, M. (2007) Cajal bodies and the nucleolus are required for a plant virus systemic infection. EMBO J. 26, 2169-2179.

Kim, V.N. (2008) Sorting Out Small RNAs. Cell 133, 25-26.

Kon, T., Sharma, P. and Ikegami, M. (2007) Suppressor of RNA silencing encoded by the monopartite tomato leaf curl Java begomovirus. Arch. Virol. 152, 1273-1282.

Kong, X., Hong, Y., Hsu, Y.F., Huang, H., Liu, X., Song, Z. and Zhu, J.K. (2020) SIZ1-Mediated SUMOylation of ROS1 Enhances Its Stability and Positively Regulates Active DNA Demethylation in Arabidopsis. Mol. Plant 13, 1816-1824.

Kørner, C.J., Klauser, D., Niehl, A., Domínguez-Ferreras, A., Chinchilla, D., Boller, T., Heinlein, M. and Hann, D.R. (2013) The Immunity Regulator BAK1 Contributes to Resistance Against Diverse RNA Viruses. Mol. Plant-Microbe Interact. 26, 1271-1280.

Kumakura, N., Takeda, A., Fujioka, Y., Motose, H., Takano, R. and Watanabe, Y. (2009) SGS3 and RDR6 interact and colocalize in cytoplasmic SGS3/RDR6-bodies. FEBS Lett. 583, 1261-1266.

Kumar, V., Mishra, S.K., Rahman, J., Taneja, J., Sundaresan, G., Mishra, N.S. and Mukherjee, S.K. (2015) Mungbean yellow mosaic Indian virus encoded AC2 protein suppresses RNA silencing by inhibiting Arabidopsis RDR6 and AGO1 activities. Virology 486, 158-172.

Kurepa, J., Walker, J.M., Smalle, J., Gosink, M.M., Davis, S.J., Durham, T.L., Sung, D.Y. and Vierstra, R.D. (2003) The small ubiquitin-like modifier (SUMO) protein modification system in Arabidopsis. Accumulation of sumo1 and -2 conjugates is increased by stress. J. Biol. Chem. 278, 6862-6872.

Kuria, P., Ilyas, M., Ateka, E., Miano, D., Onguso, J., Carrington, J.C. and Taylor, N.J. (2017) Differential response of cassava genotypes to infection by cassava mosaic geminiviruses. Virus Res. 227, 69-81.

Łabno, A., Tomecki, R. and Dziembowski, A. (2016) Cytoplasmic RNA decay pathways - Enzymes and 
mechanisms. Biochim. Biophys. Acta-Mol. Cell Res. 1863, 3125-3147.

Lal, A., Kil, E.J., Thuy, V.T.B., Fadhila, C., Ho, P.T., Byun, H.S., Dao, H.T., Kim, J.K. and Lee, S. (2020) Milk vetch dwarf virus infection in the Solanaceae and Caricaceae families in Southeast Asia. Plant Pathol. 69, 1026-1033.

Lal, A., Vo, T.T.B., Sanjaya, I.G.N.P.W., Ho, P.T., Kim, J.K., Kil, E.J. and Lee, S. (2020) Nanovirus Disease Complexes: An Emerging Threat in the Modern Era. Front. Plant Sci. 11, 558403.

Lapidot, M. (2007) Screening for Tylcv-Resistant Plants Using Whitefly-Mediated Inoculation. In Tomato Yellow Leaf Curl Virus Disease., pp. 329-342.

Lapidot, M. and Friedmann, M. (2002) Breeding for resistance to whitefly-transmitted geminivirus. Ann. Appl. Biol. 140, 109-127.

Lapidot, M., Friedmann, M., Pilowsky, M., Ben-Joseph, R. and Cohen, S. (2001) Effect of host plant resistance to Tomato yellow leaf curl virus (TYLCV) on virus acquisition and transmission by its whitefly vector. Phytopathology 91, 1209-1213.

Lapidot, M., Karniel, U., Gelbart, D., et al. (2015) A Novel Route Controlling Begomovirus Resistance by the Messenger RNA Surveillance Factor Pelota. PLoS Genet. 11, e1005538.

Lau, O.S. and Deng, X.W. (2012) The photomorphogenic repressors COP1 and DET1: 20 years later. Trends Plant Sci. 17, 584-593.

Laufs, J., Traut, W., Heyraud, F., Matzeit, V., Rogers, S.G., Schell, J. and Gronenborn, B. (1995) In vitro cleavage and joining at the viral origin of replication by the replication initiator protein of tomato yellow leaf curl virus. Proc. Natl. Acad. Sci. 92, 3879-3883.

Law, J.A. and Jacobsen, S.E. (2010) Establishing, maintaining and modifying DNA methylation patterns in plants and animals. Nat. Rev. Genet. 11, 204-220.

Law, J.A., Vashisht, A.A., Wohlschlegel, J.A. and Jacobsen, S.E. (2011) SHH1, a Homeodomain protein required for DNA Methylation, as well as RDR2, RDM4, and Chromatin remodeling factors, associate with RNA Polymerase IV. PLoS Genet. 7, e1002195.

Lee, J., Nam, J., Park, H.C., et al. (2006) Salicylic acid-mediated innate immunity in Arabidopsis is regulated by SIZ1 SUMO E3 ligase. Plant J. 49, 79-90.

Lee, J.H., Chung, D.J., Lee, J.M. and Yeam, I. (2021) Development and application of gene-specific markers for tomato yellow leaf curl virus resistance in both field and artificial infections. Plants 10, 1-18.

Lee, S., Whitaker, V.M. and Hutton, S.F. (2016) Mini Review: Potential Applications of Non-host Resistance for Crop Improvement. Front. Plant Sci. 7, 997.

Legarrea, S., Barman, A., Marchant, W., Diffie, S. and Srinivasan, R. (2015) Temporal effects of a Begomovirus infection and host plant resistance on the preference and development of an insect vector, Bemisia tabaci, and implications for epidemics. PLoS One 10, e0142114.

Leibman, D., Prakash, S., Wolf, D., et al. (2015) Immunity to tomato yellow leaf curl virus in transgenic tomato is associated with accumulation of transgene small RNA. Arch. Virol. 160, 2727-2739.

Leibman, D., Wolf, D., Saharan, V., Zelcer, A., Arazi, T., Yoel, S., Gaba, V. and Gal-On, A. (2011) A high level of transgenic viral small RNA is associated with broad potyvirus resistance in cucurbits. Mol. PlantMicrobe Interact. 24, 1220-1238.

Lewsey, M.G., Hardcastle, T.J., Melnyk, C.W., Molnar, A., Valli, A., Urich, M.A., Nery, J.R., Baulcombe, D.C. and Ecker, J.R. (2016) Mobile small RNAs regulate genome-wide DNA methylation. Proc. Natl. Acad. Sci. 113, E801-E810.

Li, C.F., Henderson, I.R., Song, L., Fedoroff, N., Lagrange, T. and Jacobsen, S.E. (2008) Dynamic regulation of ARGONAUTE4 within multiple nuclear bodies in Arabidopsis thaliana. PLoS Genet. 4, e27.

Li, C.F., Pontes, O., El-Shami, M., Henderson, I.R., Bernatavichute, Y. V., Chan, S.W.L., Lagrange, T., Pikaard, C.S. and Jacobsen, S.E. (2006) An ARGONAUTE4-Containing Nuclear Processing Center Colocalized with Cajal Bodies in Arabidopsis thaliana. Cell 126, 93-106.

Li, F., Huang, C., Li, Z. and Zhou, X. (2014) Suppression of RNA Silencing by a Plant DNA Virus Satellite Requires a Host Calmodulin-Like Protein to Repress RDR6 Expression. PLoS Pathog. 10, e1003921.

Li, F. and Wang, A. (2018) RNA decay is an antiviral defense in plants that is counteracted by viral RNA silencing suppressors. PLoS Pathog. 14, e1007228.

Li, F., Wang, Y. and Zhou, X. (2017) SGS3 cooperates with RDR6 in triggering geminivirus-induced gene silencing and in suppressing geminivirus infection in Nicotiana Benthamiana. Viruses 9, 247. 
Li, F., Xu, X., Huang, C., Gu, Z., Cao, L., Hu, T., Ding, M., Li, Z. and Zhou, X. (2015) The AC5 protein encoded by Mungbean yellow mosaic India virus is a pathogenicity determinant that suppresses RNA silencingbased antiviral defenses. New Phytol. 208, 555-569.

Li, F., Zhao, N., Li, Z., Xu, X., Wang, Y., Yang, X., Liu, S.S., Wang, A. and Zhou, X. (2017) A calmodulinlike protein suppresses RNA silencing and promotes geminivirus infection by degrading SGS3 via the autophagy pathway in Nicotiana benthamiana. PLoS Pathog. 13, e1006213.

Lim, G.-H., Hoey, T., Zhu, S., Clavel, M., Yu, K., Navarre, D., Kachroo, A., Deragon, J.-M. and Kachroo, P. (2018) COP1, a negative regulator of photomorphogenesis, positively regulates plant disease resistance via double-stranded RNA binding proteins. PLoS Pathog. 14, e1006894.

Liu, L. and Chen, X. (2016) RNA Quality Control as a Key to Suppressing RNA Silencing of Endogenous Genes in Plants. Mol. Plant 9, 826-836.

Liu, Y.L., Schiff, M. and Dinesh-Kumar, S.P. (2002) Virus-induced gene silencing in tomato. Plant J. 31, 777786.

Livak, K.J. and Schmittgen, T.D. (2001) Analysis of relative gene expression data using real-time quantitative PCR and the -2 delta delta ct method. Methods 25, 402-408.

Love, A.J., Yu, C., Petukhova, N. V., Kalinina, N.O., Chen, J. and Taliansky, M.E. (2017) Cajal bodies and their role in plant stress and disease responses. RNA Biol. 14, 779-790.

Lowrey, A.J., Cramblet, W. and Bentz, G.L. (2017) Viral manipulation of the cellular sumoylation machinery. Cell Commun. Signal. 15, 27.

Luna, A.P., Morilla, G., Voinnet, O. and Bejarano, E.R. (2012) Functional analysis of gene-silencing suppressors from tomato yellow leaf curl disease viruses. Mol. Plant-Microbe Interact. 25, 12941306.

Luo, Y.X., Han, Y.F., Zhao, Q.Y., Du, J.L., Dou, K., Li, L., Chen, S. and He, X.J. (2018) Sumoylation of SUVR2 contributes to its role in transcriptional gene silencing. Sci. China Life Sci. 61, 235-243.

Ma, X., Zhou, Y. and Moffett, P. (2019) Alterations in cellular RNA decapping dynamics affect tomato spotted wilt virus cap snatching and infection in Arabidopsis. New Phytol. 224, 789-803.

Mabvakure, B., Martin, D.P., Kraberger, S., et al. (2016) Ongoing geographical spread of Tomato yellow leaf curl virus. Virology 498, 257-264.

Madeira, F., Park, Y.M., Lee, J., et al. (2019) The EMBL-EBI search and sequence analysis tools APIs in 2019. Nucleic Acids Res. 47, W636-W641.

Mahfouz, M.M. (2010) RNA-directed DNA methylation: Mechanisms and functions. Plant Signal. Behav. 5, $806-816$.

Maio, F., Arroyo-Mateos, M., Bobay, B.G., Bejarano, E.R., Prins, M. and Burg, H.A. van den (2019) A lysine residue essential for geminivirus replication also controls nuclear localization of the TYLCV Rep protein. J. Virol. 93, e01910-18.

Mäkinen, K., Lõhmus, A. and Pollari, M. (2017) Plant RNA Regulatory Network and RNA Granules in Virus Infection. Front. Plant Sci. 8, 2093.

Mallory, A. and Vaucheret, H. (2010) Form, Function, and Regulation of ARGONAUTE Proteins. Plant Cell 22, 3879-3889.

Marchant, W.G., Gautam, S., Hutton, S.F. and Srinivasan, R. (2020) Tomato Yellow Leaf Curl Virus-Resistant and -Susceptible Tomato Genotypes Similarly Impact the Virus Population Genetics. Front. Plant Sci. 11, 599697.

Marí-Ordóñez, A., Marchais, A., Etcheverry, M., Martin, A., Colot, V. and Voinnet, O. (2013) Reconstructing de novo silencing of an active plant retrotransposon. Nat. Genet. 45, 1029-1039.

Martinez, F. and Daros, J.-A. (2014) Tobacco etch virus Protein P1 Traffics to the Nucleolus and Associates with the Host 60S Ribosomal Subunits during Infection. J. Virol. 88, 10725-10737.

Maruthi, M.N., Czosnek, H., Vidavski, F., et al. (2003) Comparison of resistance to Tomato leaf curl virus (India) and Tomato yellow leaf curl virus (Israel) among Lycopersicon wild species, breeding lines and hybrids. Eur. J. Plant Pathol. 109, 1-11.

Matic, I., Schimmel, J., Hendriks, I.A., Santen, M.A. van, Rijke, F. van de, Dam, H. van, Gnad, F., Mann, M. and Vertegaal, A.C.O. (2010) Site-Specific Identification of SUMO-2 Targets in Cells Reveals an Inverted SUMOylation Motif and a Hydrophobic Cluster SUMOylation Motif. Mol. Cell 39, 641-652.

Matzke, M., Kanno, T., Daxinger, L., Huettel, B. and Matzke, A.J. (2009) RNA-mediated chromatin-based 
silencing in plants. Curr. Opin. Cell Biol. 21, 367-376.

Matzke, M.A. and Mosher, R.A. (2014) RNA-directed DNA methylation: An epigenetic pathway of increasing complexity. Nat. Rev. Genet. 15, 394-408.

Mazur, M.J., Kwaaitaal, M., Mateos, M.A., Maio, F., Kini, R.K., Prins, M. and Burg, H.A. van den (2019) The SUMO Conjugation Complex Self-Assembles into Nuclear Bodies Independent of SIZ1 and COP1. Plant Physiol. 179, 168-183.

McCue, A.D., Panda, K., Nuthikattu, S., Choudury, S.G., Thomas, E.N. and Slotkin, R.K. (2015) ARGONAUTE 6 bridges transposable element mRNA-derived siRNAs to the establishment of DNA methylation. EMBO J. 34, 20-35.

Melnyk, C.W., Molnar, A., Bassett, A. and Baulcombe, D.C. (2011) Mobile 24 nt small RNAs direct transcriptional gene silencing in the root meristems of Arabidopsis thaliana. Curr. Biol. 21, 1678-1683.

Mermigka, G., Verret, F. and Kalantidis, K. (2016) RNA silencing movement in plants. J. Integr. Plant Biol. 58, 328-342.

Mi, S., Cai, T., Hu, Y., et al. (2008) Sorting of Small RNAs into Arabidopsis Argonaute Complexes Is Directed by the $5^{\prime}$ Terminal Nucleotide. Cell 133, 116-127.

Miller, M.J., Barrett-Wilt, G.A., Hua, Z. and Vierstra, R.D. (2010) Proteomic analyses identify a diverse array of nuclear processes affected by small ubiquitin-like modifier conjugation in Arabidopsis. Proc. Natl. Acad. Sci. 107, 16512-16517.

Miura, K., Jin, J.B. and Hasegawa, P.M. (2007) Sumoylation, a post-translational regulatory process in plants. Curr. Opin. Plant Biol. 10, 495-502.

Mlotshwa, S., Pruss, G.J., Peragine, A., Endres, M.W., Li, J., Chen, X., Poethig, R.S., Bowman, L.H. and Vance, V. (2008) Dicer-like2 plays a primary role in transitive silencing of transgenes in Arabidopsis. PLoS One 3, e1755.

Moffett, P. (2009) Mechanisms of Recognition in Dominant R Gene Mediated Resistance. In Advances in Virus Research., pp. 1-33. Elsevier.

Molnar, A., Melnyk, C.W., Bassett, A., Hardcastle, T.J., Dunn, R. and Baulcombe, D.C. (2010) Small silencing RNAs in plants are mobile and direct epigenetic modification in recipient cells. Science 328, 872-875.

Montavon, T., Kwon, Y., Zimmermann, A., Hammann, P., Vincent, T., Cognat, V., Michel, F. and Dunoyer, P. (2017) A specific dsRNA-binding protein complex selectively sequesters endogenous inverted-repeat siRNA precursors and inhibits their processing. Nucleic Acids Res. 45, 1330-1344.

Morales, F.J. and Anderson, P.K. (2001) The emergence and dissemination of whitefly-transmitted geminiviruses in Latin America. Arch. Virol. 146, 415-441.

Moreno, A.B., Alba, A.E.M. De, Bardou, F., Crespi, M.D., Vaucheret, H., Maizel, A. and Mallory, A.C. (2013) Cytoplasmic and nuclear quality control and turnover of single-stranded RNA modulate posttranscriptional gene silencing in plants. Nucleic Acids Res. 41, 4699-4708.

Morilla, G., Janssen, D., García-Andrés, S., Moriones, E., Cuadrado, I.M. and Bejarano, E.R. (2005) Pepper (Capsicum annuum) Is a Dead-End Host for Tomato yellow leaf curl virus. Phytopathology 95, 10891097.

Morilla, G., Krenz, B., Jeske, H., Bejarano, E.R. and Wege, C. (2004) Tête à Tête of Tomato Yellow Leaf Curl Virus and Tomato Yellow Leaf Curl Sardinia Virus in Single Nuclei. J. Virol. 78, 10715-10723.

Moriones, E. and Navas-Castillo, J. (2000) Tomato yellow leaf curl virus, an emerging virus complex causing epidemics worldwide. Virus Res. 71, 123-134.

Morris, G.E. (2008) The Cajal body. Biochim. Biophys. Acta-Mol. Cell Res. 1783, 2108-2115.

Mubin, M., Briddon, R.W. and Mansoor, S. (2019) The V2 protein encoded by a monopartite begomovirus is a suppressor of both post-transcriptional and transcriptional gene silencing activity. Gene 686, 43-48.

Muhammad, T., Zhang, F., Zhang, Y. and Liang, Y. (2019) RNA Interference: A Natural Immune System of Plants to Counteract Biotic Stressors. Cells 8, 38.

Mundt, C.C. (2018) Pyramiding for resistance durability: Theory and practice. Phytopathology 108, 792-802.

Nagar, S., Pedersen, T.J., Carrick, K.M., Hanley-Bowdoin, L. and Robertson, D. (1995) A geminivirus induces expression of a host DNA synthesis protein in terminally differentiated plant cells. Plant Cell 7, 705719.

Nakagawa, T., Suzuki, T., Murata, S., et al. (2007) Improved gateway binary vectors: High-performance vectors for creation of fusion constructs in transgenic analysis of plants. Biosci. Biotechnol. Biochem. 
71, 2095-2100.

Nakamura, S., Mano, S., Tanaka, Y., et al. (2010) Gateway binary vectors with the bialaphos resistance gene, bar, as a selection marker for plant transformation. Biosci. Biotechnol. Biochem. 74, 1315-1319.

Nakasugi, K., Crowhurst, R.N., Bally, J., Wood, C.C., Hellens, R.P. and Waterhouse, P.M. (2013) De Novo Transcriptome Sequence Assembly and Analysis of RNA Silencing Genes of Nicotiana benthamiana. PLoS One 8, e59534.

Navarro, L., Jay, F., Nomura, K., He, S.Y. and Voinnet, O. (2008) Suppression of the microRNA pathway by bacterial effector proteins. Science 321, 964-967.

Nawaz-ul-Rehman, M.S. and Fauquet, C.M. (2009) Evolution of geminiviruses and their satellites. FEBS Lett. 583, 1825-1832.

Nawaz-ul-Rehman, M.S., Nahid, N., Mansoor, S., Briddon, R.W. and Fauquet, C.M. (2010) Posttranscriptional gene silencing suppressor activity of two non-pathogenic alphasatellites associated with a begomovirus. Virology 405, 300-308.

Nicaise, V. and Candresse, T. (2017) Plum pox virus capsid protein suppresses plant pathogen-associated molecular pattern (PAMP)-triggered immunity. Mol. Plant Pathol. 18, 878-886.

Nicaise, V., Gallois, J., Chafiai, F., et al. (2007) Coordinated and selective recruitment of elF4E and elF4G factors for potyvirus infection in Arabidopsis thaliana. FEBS Lett. 581, 1041-1046.

Niehl, A., Wyrsch, I., Boller, T. and Heinlein, M. (2016) Double-stranded RNAs induce a pattern-triggered immune signaling pathway in plants. New Phytol. 211, 1008-1019.

Noris, E., Vaira, A.M., Caciagli, P., Masenga, V., Gronenborn, B. and Accotto, G.P. (1998) Amino Acids in the Capsid Protein of Tomato Yellow Leaf Curl Virus That Are Crucial for Systemic Infection, Particle Formation, and Insect Transmission. J. Virol. 72, 10050-10057.

Nuthikattu, S., McCue, A.D., Panda, K., Fultz, D., DeFraia, C., Thomas, E.N. and Slotkin, R.K. (2013) The initiation of epigenetic silencing of active transposable elements is triggered by RDR6 and 21-22 nucleotide small interfering RNAs. Plant Physiol. 162, 116-131.

Ohnishi, J., Yamaguchi, H. and Saito, A. (2016) Analysis of the Mild strain of tomato yellow leaf curl virus, which overcomes Ty-2 gene - mediated resistance in tomato line H24. Arch. Virol. 161, 2207-2217.

Okano, Y., Senshu, H., Hashimoto, M., et al. (2014) In Planta Recognition of a Double-Stranded RNA Synthesis Protein Complex by a Potexviral RNA Silencing Suppressor. Plant Cell 26, 2168-2183.

Oliveira, A.S. De, Koolhaas, I., Boiteux, L.S., Caldararu, O.F., Petrescu, A.J., Resende, R.O. and Kormelink, R. (2016) Cell death triggering and effector recognition by Sw-5 SD-CNL proteins from resistant and susceptible tomato isolines to Tomato spotted wilt virus. Mol. Plant Pathol. 17, 1442-1454.

Osterlund, M.T., Hardtke, C.S., Wei, N. and Deng, X.W. (2000) Targeted destabilization of HY5 during lightregulated development of Arabidopsis. Nature 405, 462-466.

Padidam, M., Beachy, R. and Fauquet, C. (1995) Classification and identification of geminiviruses using sequence comparisons. J. Gen. Virol. 76, 249-263.

Pakkianathan, B.C., Kontsedalov, S., Lebedev, G., Mahadav, A., Zeidan, M., Czosnek, H. and Ghanim, M. (2015) Replication of Tomato Yellow Leaf Curl Virus in Its Whitefly Vector, Bemisia tabaci. J. Virol. 89, 9791-9803.

Palumbo, J.C., Horowitz, A.R. and Prabhaker, N. (2001) Insecticidal control and resistance management for Bemisia tabaci. Crop Prot. 20, 739-765.

Panda, K. and Slotkin, R.K. (2013) Proposed mechanism for the initiation of transposable element silencing by the RDR6-directed DNA methylation pathway. Plant Signal. Behav. 8, e25206.

Panno, S., Caruso, A.G. and Davino, S. (2018) The nucleotide sequence of a recombinant tomato yellow leaf curl virus strain frequently detected in Sicily isolated from tomato plants carrying the Ty-1 resistance gene. Arch. Virol. 163, 795-797.

Parent, J.S., Bouteiller, N., Elmayan, T. and Vaucheret, H. (2015) Respective contributions of Arabidopsis DCL2 and DCL4 to RNA silencing. Plant J. 81, 223-232.

Pavan, S., Jacobsen, E., Visser, R.G.F. and Bai, Y. (2010) Loss of susceptibility as a novel breeding strategy for durable and broad-spectrum resistance. Mol. Breed. 25, 1-12.

Pavet, V., Quintero, C., Cecchini, N.M., Rosa, A.L. and Alvarez, M.E. (2006) Arabidopsis displays centromeric DNA hypomethylation and cytological alterations of heterochromatin upon attack by Pseudomonas syringae. Mol. Plant-Microbe Interact. 19, 577-587. 
Pérez-Cañamás, M., Blanco-Pérez, M., Forment, J. and Hernández, C. (2017) Nicotiana benthamiana plants asymptomatically infected by Pelargonium line pattern virus show unusually high accumulation of viral small RNAs that is neither associated with DCL induction nor RDR6 activity. Virology 501, 136146.

Pérez De Castro, A., Díez, M.J. and Nuez Viñals, F. (2008) Exploiting partial resistance to Tomato yellow leaf curl virus derived from Solanum pimpinellifolium UPV16991. Plant Dis. 92, 1083-1090.

Pfander, B., Moldovan, G.L., Sacher, M., Hoege, C. and Jentsch, S. (2005) SUMO-modified PCNA recruits Srs2 to prevent recombination during S phase. Nature 436, 428-433.

Picó, B., Díez, M.J. and Nuez, F. (1996) Viral diseases causing the greatest economic losses to the tomato crop. II. The tomato yellow leaf curl virus - A review. Sci. Hortic. (Amsterdam). 67, 151-196.

Picó, B., Ferriol, M., Díez, M.J. and Nuez, F. (1999) Developing tomato breeding lines resistant to tomato yellow leaf curl virus. Plant Breed. 118, 537-542.

Picó, B., Sifres, A., Elia, M., Diez, M.J. and Nuez, F. (2000) Searching for new resistance sources to tomato yellow leaf curl virus within a highly variable wild Lycopersicon genetic pool. Acta Physiol. Plant. 22, 344-350.

Piedra-Aguilera, Á., Jiao, C., Luna, A.P., et al. (2019) Integrated single-base resolution maps of transcriptome, sRNAome and methylome of Tomato yellow leaf curl virus (TYLCV) in tomato. Sci. Rep. 9, 2863.

Pietersen, G. and Smith, M.F. (2002) Tomato yellow leaf curl virus Resistant Tomatoes Show Resistance to Tomato curly stunt virus. Plant Dis. 86, 528-534.

Pilartz, M. and Jeske, H. (1992) Abutilon mosaic geminivirus double-stranded DNA is packed into minichromosomes. Virology 189, 800-802.

Podolec, R. and Ulm, R. (2018) Photoreceptor-mediated regulation of the COP1/SPA E3 ubiquitin ligase. Curr. Opin. Plant Biol. 45, 18-25.

Polston, J.E. and Anderson, P.K. (1997) The emergence of whitefly-transmitted geminiviruses in tomato in the western hemisphere. Plant Dis. 81, 1358-1369.

Pontes, O., Li, C.F., Nunes, P.C., Haag, J., Ream, T., Vitins, A., Jacobsen, S.E. and Pikaard, C.S. (2006) The Arabidopsis Chromatin-Modifying Nuclear siRNA Pathway Involves a Nucleolar RNA Processing Center. Cell 126, 79-92.

Pontes, O. and Pikaard, C.S. (2008) siRNA and miRNA processing: new functions for Cajal bodies. Curr. Opin. Genet. Dev. 18, 197-203.

Pontes, O., Vitins, A., Ream, T.S., Hong, E., Pikaard, C.S. and Costa-Nunes, P. (2013) Intersection of Small RNA Pathways in Arabidopsis thaliana Sub-Nuclear Domains. PLoS One 8, e65652.

Pontier, D., Picart, C., Roudier, F., et al. (2012) NERD, a Plant-Specific GW Protein, Defines an Additional RNAi-Dependent Chromatin-Based Pathway in Arabidopsis. Mol. Cell 48, 121-132.

Pooggin, M.M. (2013) How can plant DNA viruses evade siRNA-directed DNA methylation and silencing? Int. J. Mol. Sci. 14, 15233-15259.

Powell, M.E., Cuthbertson, A.G.S., Bell, H.A., Boonham, N., Morris, J. and Northing, P. (2012) First record of the Q Biotype of the sweetpotato whitefly, Bemisia tabaci, intercepted in the UK. Eur. J. Plant Pathol. 133, 797-801.

Prasanna, H.C., Sinha, D.P., Rai, G.K., Krishna, R., Kashyap, S.P., Singh, N.K., Singh, M. and Malathi, V.G. (2015) Pyramiding Ty-2 and Ty-3 genes for resistance to monopartite and bipartite tomato leaf curl viruses of India. Plant Pathol. 64, 256-264.

Priyadarshini, C.G.P., Ambika, M. V., Tippeswamy, R. and Savithri, H.S. (2011) Functional characterization of coat protein and V2 involved in cell to cell movement of cotton leaf curl Kokhran Virus-Dabawali. PLoS One 6, e26929.

Pumplin, N., Sarazin, A., Jullien, P.E., Bologna, N.G., Oberlin, S. and Voinnet, O. (2016) DNA methylation influences the expression of DICER-LIKE4 isoforms, which encode proteins of alternative localization and function. Plant Cell 28, 2786-2804.

Pumplin, N. and Voinnet, O. (2013) RNA silencing suppression by plant pathogens: Defence, counterdefence and counter-counter-defence. Nat. Rev. Microbiol. 11, 745-760.

Qi, X., Bao, F.S. and Xie, Z. (2009) Small RNA deep sequencing reveals role for Arabidopsis thaliana RNAdependent RNA polymerases in viral siRNA biogenesis. PLoS One 4, e4971.

Qi, Y., He, X., Wang, X.-J., Kohany, O., Jurka, J. and Hannon, G.J. (2006) Distinct catalytic and non-catalytic 
roles of ARGONAUTE4 in RNA-directed DNA methylation. Nature 443, 1008-1012.

Qu, F. (2010) Antiviral role of plant-encoded RNA-Dependent RNA polymerases revisited with deep sequencing of small interfering RNAs of virus origin. Mol. Plant-Microbe Interact. 23, 1248-1252.

Raja, P., Jackel, J.N., Li, S., Heard, I.M. and Bisaro, D.M. (2014) Arabidopsis Double-Stranded RNA Binding Protein DRB3 Participates in Methylation-Mediated Defense against Geminiviruses. J. Virol. 88, 26112622.

Raja, P., Sanville, B.C., Buchmann, R.C. and Bisaro, D.M. (2008) Viral Genome Methylation as an Epigenetic Defense against Geminiviruses. J. Virol. 82, 8997-9007.

Raja, P., Wolf, J.N. and Bisaro, D.M. (2010) RNA silencing directed against geminiviruses: Post-transcriptional and epigenetic components. Biochim. Biophys. Acta - Gene Regul. Mech. 1799, 337-351.

Rakhshandehroo, F., Takeshita, M., Squires, J. and Palukaitis, P. (2009) The influence of RNA-dependent RNA polymerase 1 on Potato virus $Y$ infection and on other antiviral response genes. Mol. PlantMicrobe Interact. 22, 1312-1318.

Ramesh, S. V., Sahu, P.P., Prasad, M., Praveen, S. and Pappu, H.R. (2017) Geminiviruses and plant hosts: A closer examination of the molecular arms race. Viruses $\mathbf{9}, 256$.

Ramesh, S. V., Shivakumar, M., Ramteke, R., et al. (2019) Quantification of a legume begomovirus to evaluate soybean genotypes for resistance to yellow mosaic disease. J. Virol. Methods 268, 24-31.

Ratcliff, F., Martin-Hernandez, A.M. and Baulcombe, D.C. (2001) Technical Advance. Tobacco rattle virus as a vector for analysis of gene function by silencing. Plant J. 25, 237-45.

Ren, B., Guo, Y., Gao, F., Zhou, P., Wu, F., Meng, Z., Wei, C. and Li, Y. (2010) Multiple Functions of Rice Dwarf Phytoreovirus Pns10 in Suppressing Systemic RNA Silencing [. J. Virol. 84, 12914-12923.

Rishishwar, R. and Dasgupta, I. (2019) Suppressors of RNA silencing encoded by geminiviruses and associated DNA satellites. VirusDisease 30, 58-65.

Robaglia, C. and Caranta, C. (2006) Translation initiation factors: a weak link in plant RNA virus infection. Trends Plant Sci. 11, 40-45.

Rodríguez-Negrete, E., Lozano-Durán, R., Piedra-Aguilera, A., Cruzado, L., Bejarano, E.R. and Castillo, A.G. (2013) Geminivirus Rep protein interferes with the plant DNA methylation machinery and suppresses transcriptional gene silencing. New Phytol. 199, 464-475.

Rodriguez, M.S., Dargemont, C. and Hay, R.T. (2001) SUMO-1 Conjugation in Vivo Requires Both a Consensus Modification Motif and Nuclear Targeting. J. Biol. Chem. 276, 12654-12659.

Rojas, M.R., Jiang, H., Salati, R., Xoconostle-Cázares, B., Sudarshana, M.R., Lucas, W.J. and Gilbertson, R.L. (2001) Functional analysis of proteins involved in movement of the monopartite begomovirus, Tomato yellow leaf curl virus. Virology 291, 110-125.

Ronde, D. de, Butterbach, P. and Kormelink, R. (2014) Dominant resistance against plant viruses. Front. Plant Sci. 5, 1-17.

Ronde, D. de, Butterbach, P., Lohuis, D., Hedil, M., Lent, J.W.M. van and Kormelink, R. (2013) Tsw genebased resistance is triggered by a functional RNA silencing suppressor protein of the Tomato spotted wilt virus. Mol. Plant Pathol. 14, 405-415.

Rossi, J.J. (2005) Mammalian Dicer finds a partner. EMBO Rep. 6, 927-929.

Rytz, T.C., Miller, M.J., McLoughlin, F., et al. (2018) SUMOylome profiling reveals a diverse array of nuclear targets modified by the SUMO ligase SIZ1 during heat stress. Plant Cell 30, 1077-1099.

Saeed, M., Briddon, R.W., Dalakouras, A., Krczal, G. and Wassenegger, M. (2015) Functional analysis of cotton leaf curl kokhran virus/cotton leaf curl multan betasatellite RNA silencing suppressors. Biology (Basel). 4, 697-714.

Safarnejad, M.R., Fischer, R. and Commandeur, U. (2009) Recombinant-antibody-mediated resistance against Tomato yellow leaf curl virus in Nicotiana benthamiana. Arch. Virol. 154, 457-467.

Sahin, U., Lapaquette, P., Andrieux, A., Faure, G. and Dejean, A. (2014) Sumoylation of human argonaute 2 at lysine-402 regulates its stability. PLoS One 9, e102957.

Saijo, Y., Loo, E.P. and Yasuda, S. (2018) Pattern recognition receptors and signaling in plant-microbe interactions. Plant J. 93, 592-613.

Saijo, Y., Sullivan, J.A., Wang, H., Yang, J., Shen, Y., Rubio, V., Ma, L., Hoecker, U. and Deng, X.W. (2003) The COP1-SPA1 interaction defines a critical step in phytochrome A-mediated regulation of HY5 activity. Genes Dev. 17, 2642-2647. 
Saleh, A., Withers, J., Mohan, R., et al. (2015) Posttranslational Modifications of the Master Transcriptional Regulator NPR1 Enable Dynamic but Tight Control of Plant Immune Responses. Cell Host Microbe 18, 169-182.

Sampson, D.A., Wang, M. and Matunis, M.J. (2001) The Small Ubiquitin-like Modifier-1 (SUMO-1) Consensus Sequence Mediates Ubc9 Binding and is Essential for SUMO-1 Modification. J. Biol. Chem. 276, 21664-21669.

Sánchez-Campos, S., Rodríguez-Negrete, E.A., Cruzado, L., Grande-Pérez, A., Bejarano, E.R., Navas-Castillo, J. and Moriones, E. (2016) Tomato yellow leaf curl virus: No evidence for replication in the insect vector Bemisia tabaci. Sci. Rep. 6, 30942.

Sanchez-Duran, M.A., Dallas, M.B., Ascencio-Ibanez, J.T., Reyes, M.I., Arroyo-Mateos, M., Ruiz-Albert, J., Hanley-Bowdoin, L. and Bejarano, E.R. (2011) Interaction between Geminivirus Replication Protein and the SUMO-Conjugating Enzyme Is Required for Viral Infection. J. Virol. 85, 9789-9800.

Saunders, K., Bedford, I.D., Briddon, R.W., Markham, P.G., Wong, S.M. and Stanley, J. (2000) A unique virus complex causes Ageratum yellow vein disease. Proc. Natl. Acad. Sci. 97, 6890-6895.

Saunders, K., Lucy, A. and Stanley, J. (1992) RNA-Primed Complementary-Sense DNA-Synthesis of the Geminivirus African Cassava Mosaic-Virus. Nucleic Acids Res. 20, 6311-6315.

Schie, C.C.N. van and Takken, F.L.W. (2014) Susceptibility Genes 101: How to Be a Good Host. Annu. Rev. Phytopathol. 52, 551-581.

Schnettler, E., Hemmes, H., Huismann, R., Goldbach, R., Prins, M. and Kormelink, R. (2010) Diverging Affinity of Tospovirus RNA Silencing Suppressor Proteins, NSs, for Various RNA Duplex Molecules. J. Virol. 84, 11542-11554.

Schnettler, E., Sterken, M.G., Leung, J.Y., et al. (2012) Noncoding Flavivirus RNA Displays RNA Interference Suppressor Activity in Insect and Mammalian Cells. J. Virol. 86, 13486-13500.

Schwach, F., Vaistij, F. abian E., Jones, L. and Baulcombe, D.C. (2005) An RNA-Dependent RNA Polymerase Prevents Meristem Invasion by Potato Virus $X$ and Is Required for the Activity But Not the Production of a Systemic Silencing Signal. Plant Physiol. 138, 1842-1852.

Schwartz, M., Chen, J., Janda, M., Sullivan, M., Boon, J. Den and Ahlquist, P. (2002) A positive-strand RNA virus replication complex parallels form and function of retrovirus capsids. Mol. Cell 9, 505-514.

Selth, L.A., Randles, J.W. and Rezaian, M.A. (2004) Host responses to transient expression of individual genes encoded by Tomato leaf curl virus. Mol. Plant-Microbe Interact. 17, 27-33.

Seo, H.S., Watanabe, E., Tokutomi, S., Nagatani, A. and Chua, N.H. (2004) Photoreceptor ubiquitination by COP1 E3 ligase desensitizes phytochrome A signaling. Genes Dev. 18, 617-622.

Seo, H.S., Yang, J.-Y., Ishikawa, M., Bolle, C., Ballesteros, M.L. and Chua, N.-H. (2003) LAF1 ubiquitination by COP1 controls photomorphogenesis and is stimulated by SPA1. Nature 423, 995-999.

Settlage, S.B., See, R.G. and Hanley-Bowdoin, L. (2005) Geminivirus C3 protein: replication enhancement and protein interactions. J. Virol. 79, 9885-9895.

Shahid, M.S., Ito, T., Kimbara, J., Onozato, A., Natsuaki, K.T. and Ikegami, M. (2013) Evaluation of Tomato Hybrids Carrying Ty-1 and Ty-2 Loci to Japanese Monopartite Begomovirus Species. J. Phytopathol. 161, 205-209.

Sharma, P. and Ikegami, M. (2010) Tomato leaf curl Java virus V2 protein is a determinant of virulence, hypersensitive response and suppression of posttranscriptional gene silencing. Virology 396, 85-93.

Sharma, P., Ikegami, M. and Kon, T. (2010) Identification of the virulence factors and suppressors of posttranscriptional gene silencing encoded by Ageratum yellow vein virus, a monopartite begomovirus. Virus Res. 149, 19-27.

Shaw, J., Love, A.J., Makarova, S.S., Kalinina, N.O., Harrison, B.D. and Taliansky, M.E. (2014) Coilin, the signature protein of Cajal bodies, differentially modulates the interactions of plants with viruses in widely different taxa. Nucleus 5, 85-94.

Shen, X., Yan, Z., Wang, X., et al. (2020) The NLR Protein Encoded by the Resistance Gene Ty-2 Is Triggered by the Replication-Associated Protein Rep/C1 of Tomato Yellow Leaf Curl Virus. Front. Plant Sci. 11, 545306.

Shivaprasad, P. V., Akbergenov, R., Trinks, D., Rajeswaran, R., Veluthambi, K., Hohn, T. and Pooggin, M.M. (2005) Promoters, Transcripts, and Regulatory Proteins of Mungbean Yellow Mosaic Geminivirus. J. Virol. 79, 8149-8163. 
Shoemaker, C.J. and Green, R. (2012) Translation drives mRNA quality control. Nat. Struct. Mol. Biol. 19, 594-601.

Soitamo, A.J., Jada, B. and Lehto, K. (2012) Expression of geminiviral AC2 RNA silencing suppressor changes sugar and jasmonate responsive gene expression in transgenic tobacco plants. BMC Plant Biol. 12, 204.

Srinivasan, R., Riley, D., Diffie, S., Sparks, A. and Adkins, S. (2012) Whitefly population dynamics and evaluation of whitefly-transmitted tomato yellow leaf curl virus (TYLCV)-resistant tomato genotypes as whitefly and TYLCV reservoirs. J. Econ. Entomol. 105, 1447-1456.

Stacey, M.G. and Arnim, A.G. Von (1999) A novel motif mediates the targeting of the Arabidopsis COP1 protein to subnuclear foci. J. Biol. Chem. 274, 27231-27236.

Stacey, M.G., Hicks, S.N. and Arnim, A.G. Von (1999) Discrete domains mediate the light-responsive nuclear and cytoplasmic localization of Arabidopsis COP1. Plant Cell 11, 349-363.

Stanley, J. (1995) Analysis of African cassava mosaic virus recombinants suggests strand nicking occurs withinthe conserved nonanucleotide motif during the initiation of rolling circle DNA replication. Virology 206, 707-712.

Stanley, J., Markham, P.G., Callis, R.J. and Pinner, M.S. (1986) The nucleotide sequence of an infectious clone of the geminivirus beet curly top virus. EMBO J. 5, 1761-1767.

Stenger, D.C., Revington, G.N., Stevenson, M.C. and Bisaro, D.M. (1991) Replicational release of geminivirus genomes from tandemly repeated copies: evidence for rolling-circle replication of a plant viral DNA. Proc. Natl. Acad. Sci. 88, 8029-8033.

Stroud, H., Greenberg, M.V.C., Feng, S., Bernatavichute, Y. V. and Jacobsen, S.E. (2013) Comprehensive analysis of silencing mutants reveals complex regulation of the Arabidopsis methylome. Cell 152, 352-364.

Sun, Y.W., Tee, C.S., Ma, Y.H., Wang, G., Yao, X.M. and Ye, J. (2015) Attenuation of Histone Methyltransferase KRYPTONITE-mediated transcriptional gene silencing by Geminivirus. Sci. Rep. 5, 16476.

Sunitha, S., Shanmugapriya, G., Balamani, V. and Veluthambi, K. (2013) Mungbean yellow mosaic virus (MYMV) AC4 suppresses post-transcriptional gene silencing and an AC4 hairpin RNA gene reduces MYMV DNA accumulation in transgenic tobacco. Virus Genes 46, 496-504.

Sunter, G. and Bisaro, D.M. (1997) Regulation of a geminivirus coat protein promoter by AL2 protein (TrAP): Evidence for activation and derepression mechanisms. Virology 232, 269-280.

Sunter, G. and Bisaro, D.M. (1991) Transactivation in a geminivirus: AL2 gene product is needed for coat protein expression. Virology 180, 416-419.

Sunter, G., Hartitz, M.D., Hormuzdi, S.G., Brough, C.L. and Bisaro, D.M. (1990) Genetic analysis of tomato golden mosaic virus: ORF AL2 is required for coat protein accumulation while ORF AL3 is necessary for efficient DNA replication. Virology 179, 69-77.

Taochy, C., Gursanscky, N.R., Cao, J., et al. (2017) A genetic screen for impaired systemic RNAi highlights the crucial role of DICER-LIKE 2. Plant Physiol. 175, 1424-1437.

Teng, K., Chen, H., Lai, J., Zhang, Z., Fang, Y., Xia, R., Zhou, X., Guo, H. and Xie, Q. (2010) Involvement of C4 protein of beet severe curly top virus (Family Geminiviridae) in virus movement. PLoS One 5, e11280.

Thomas, J.E., Gronenborn, B., Harding, R.M., et al. (2021) ICTV Virus Taxonomy Profile: Nanoviridae. J. Gen. Virol., 1-2.

Thran, M., Link, K. and Sonnewald, U. (2012) The Arabidopsis DCP2 gene is required for proper mRNA turnover and prevents transgene silencing in Arabidopsis. Plant J. 72, 368-377.

Tiwari, N., Sharma, P.K. and Malathi, V.G. (2013) Functional characterization of $\beta c 1$ gene of Cotton leaf curl Multan betasatellite. Virus Genes 46, 111-119.

Tomás, D.M., Cañizares, M.C., Abad, J., Fernández-Muñoz, R. and Moriones, E. (2011) Resistance to Tomato yellow leaf curl virus Accumulation in the Tomato Wild Relative Solanum habrochaites Associated with the C4 Viral Protein. MPMI 24, 849-861.

Torre, C., Agüero, J. and Aranda, M.A. (2019) First Evidence of Tomato Yellow Leaf Curl Virus-Israel IS76 Recombinant Isolates Associated with Severe Yellow Leaf Curl Epidemics in Resistant Tomatoes in Spain. Plant Dis. 103, 780-781.

Torre, C., Donaire, L., Gómez-Aix, C., Juárez, M., Peterschmitt, M., Urbino, C., Hernando, Y., Agüero, J. and Aranda, M.A. (2018) Characterization of Begomoviruses Sampled during Severe Epidemics in Tomato 
Cultivars Carrying the Ty-1 Gene. Int. J. Mol. Sci. 19, 2614.

Trinks, D., Rajeswaran, R., Shivaprasad, P. V., Akbergenov, R., Oakeley, E.J., Veluthambi, K., Hohn, T. and Pooggin, M.M. (2005) Suppression of RNA Silencing by a Geminivirus Nuclear Protein, AC2, Correlates with Transactivation of Host Genes. J. Virol. 79, 2517-2527.

Tsuzuki, M., Motomura, K., Kumakura, N. and Takeda, A. (2017) Interconnections between mRNA degradation and RDR-dependent siRNA production in mRNA turnover in plants. J. Plant Res. 130, 211-226.

Ulrey, C.L., Liu, L., Andrews, L.G. and Tollefsbol, T.O. (2005) The impact of metabolism on DNA methylation. Hum. Mol. Genet. 14, R139-R147.

Urbino, C., Regragui, Z.F., Granier, M. and Peterschmitt, M. (2020) Fitness advantage of inter-species TYLCV recombinants induced by beneficial intra-genomic interactions rather than by specific mutations. Virology 542, 20-27.

Vanitharani, R., Chellappan, P., Pita, J.S. and Fauquet, C.M. (2004) Differential Roles of AC2 and AC4 of Cassava Geminiviruses in Mediating Synergism and Suppression of Posttranscriptional Gene Silencing. J. Virol. 78, 9487-9498.

Vargason, J.M., Szittya, G., Burgyán, J. and Tanaka Hall, T.M. (2003) Size Selective Recognition of siRNA by an RNA Silencing Suppressor. Cell 115, 799-811.

Varma, A. and Malathi, V.G. (2003) Emerging geminivirus problems: A serious threat to crop production. Ann. Appl. Biol. 142, 145-164.

Vázquez-Barrios, V., Boege, K., Sosa-Fuentes, T.G., Rojas, P. and Wegier, A. (2021) Ongoing ecological and evolutionary consequences by the presence of transgenes in a wild cotton population. Sci. Rep. 11, 1959.

Verlaan, M.G., Hutton, S.F., Ibrahem, R.M., Kormelink, R., Visser, R.G.F., Scott, J.W., Edwards, J.D. and Bai, Y. (2013) The Tomato Yellow Leaf Curl Virus Resistance Genes Ty-1 and Ty-3 Are Allelic and Code for DFDGD-Class RNA-Dependent RNA Polymerases. PLoS Genet. 9, e1003399.

Verma, V., Croley, F. and Sadanandom, A. (2018) Fifty shades of SUMO: its role in immunity and at the fulcrum of the growth-defence balance. Mol. Plant Pathol. 19, 1537-1544.

Vetten, H.J., Knierim, D., Rakoski, M.S., Menzel, W., Maiss, E., Gronenborn, B., Winter, S. and Krenz, B. (2019) Identification of a novel nanovirus in parsley. Arch. Virol. 164, 1883-1887.

Vogel, F., Hofius, D., Paulus, K.E., Jungkunz, I. and Sonnewald, U. (2011) The second face of a known player: Arabidopsis silencing suppressor AtXRN4 acts organ-specifically. New Phytol. 189, 484-493.

Voinnet, O., Pinto, Y.M. and Baulcombe, D.C. (1999) Suppression of gene silencing: A general strategy used by diverse DNA and RNA viruses of plants. Proc. Natl. Acad. Sci. 96, 14147-14152.

Voorburg, C.M., Yan, Z., Bergua-Vidal, M., Wolters, A.A., Bai, Y. and Kormelink, R. (2020) Ty-1, a universal resistance gene against geminiviruses that is compromised by co-replication of a betasatellite. Mol. Plant Pathol. 21, 160-172.

Wang, B., Li, F., Huang, C., Yang, X., Qian, Y., Xie, Y. and Zhou, X. (2014) V2 of tomato yellow leaf curl virus can suppress methylation-mediated transcriptional gene silencing in plants. J. Gen. Virol. 95, 225-230.

Wang, B., Yang, X., Wang, Y., Xie, Y. and Zhou, X. (2018) Tomato Yellow Leaf Curl Virus V2 Interacts with Host Histone Deacetylase 6 To Suppress Methylation-Mediated Transcriptional Gene Silencing in Plants. J. Virol. 92, e00036-18.

Wang, D., Zhang, Z., O'Loughlin, E., Lee, T., Houel, S., O'Carroll, D., Tarakhovsky, A., Ahn, N.G. and Yi, R. (2012) Quantitative functions of argonaute proteins in mammalian development. Genes Dev. 26, 693-704.

Wang, H., Buckley, K.J., Yang, X., Buchmann, R.C. and Bisaro, D.M. (2005) Adenosine Kinase Inhibition and Suppression of RNA Silencing by Geminivirus AL2 and L2 Proteins. J. Virol. 79, 7410-7418.

Wang, H., Hao, L., Shung, C.-Y., Sunter, G. and Bisaro, D.M. (2003) Adenosine Kinase Is Inactivated by Geminivirus AL2 and L2 Proteins. Plant Cell 15, 3020-3032.

Wang, L., Ding, Y., He, L., Zhang, G., Zhu, J.K. and Lozano-Duran, R. (2020) A virus-encoded protein suppresses methylation of the viral genome through its interaction with AGO4 in the cajal body. Elife 9, e55542.

Wang, L., Tan, H., Wu, M., Jimenez-Gongora, T., Tan, L. and Lozano-Duran, R. (2017) Dynamic Virus- 
Dependent Subnuclear Localization of the Capsid Protein from a Geminivirus. Front. Plant Sci. 8, 2165.

Wang, N., Zhang, D., Wang, Z., et al. (2014) Mutation of the RDR1 gene caused genome-wide changes in gene expression, regional variation in small RNA clusters and localized alteration in DNA methylation in rice. BMC Plant Biol. 14, 177.

Wang, T., Deng, Z., Zhang, X., et al. (2018) Tomato DCL2b is required for the biosynthesis of 22-nt small RNAs, the resulting secondary siRNAs, and the host defense against ToMV. Hortic. Res. 5, 62.

Wang, X.-B., Wu, Q., Ito, T., Cillo, F., Li, W.-X., Chen, X., Yu, J.-L. and Ding, S.-W. (2010) RNAi-mediated viral immunity requires amplification of virus-derived siRNAs in Arabidopsis thaliana. Proc. Natl. Acad. Sci. 107, 484-489.

Wang, X.B., Jovel, J., Udomporn, P., Wang, Y., Wu, Q., Li, W.X., Gasciolli, V., Vaucheret, H. and Ding, S.W. (2011) The 21-nucleotide, but not 22-nucleotide, viral secondary small interfering RNAs direct potent antiviral defense by two cooperative argonautes in Arabidopsis thaliana. Plant Cell 23, 1625-1638.

Wang, Y., Dang, M., Hou, H., Mei, Y., Qian, Y. and Zhou, X. (2014) Identification of an RNA silencing suppressor encoded by a mastrevirus. J. Gen. Virol. 95, 2082-2088.

Wang, Y., Wu, Y., Gong, Q., et al. (2019) Geminiviral V2 Protein Suppresses Transcriptional Gene Silencing through Interaction with AGO4. J. Virol. 93, e01675-18.

Wartig, L., Kheyr-Pour, A., Noris, E., Kouchkovsky, F. De, Jouanneau, F., Gronenborn, B. and Jupin, I. (1997) Genetic analysis of the monopartite tomato yellow leaf curl geminivirus: Roles of V1, V2, and C2 ORFs in viral pathogenesis. Virology 228, 132-140.

Wassenegger, M. and Krczal, G. (2006) Nomenclature and functions of RNA-directed RNA polymerases. Trends Plant Sci. 11, 142-151.

Wezel, R. Van, Dong, X., Liu, H., Tien, P., Stanley, J. and Hong, Y. (2002) Mutation of Three Cysteine Residues in Tomato yellow leaf curl virus-China C2 Protein Causes Dysfunction in Pathogenesis and Posttranscriptional Gene - Silencing Suppression. Mol. Plant-Microbe Interact. 15, 203-208.

Wezel, R. Van, Liu, H., Tien, P., Stanley, J. and Hong, Y. (2001) Gene C2 of the monopartite geminivirus Tomato yellow leaf curl virus-China encodes a pathogenicity determinant that is localized in the nucleus. Mol. Plant-Microbe Interact. 14, 1125-1128.

Willmann, M.R., Endres, M.W., Cook, R.T. and Gregory, B.D. (2011) The Functions of RNA-Dependent RNA Polymerases in Arabidopsis. Arab. B. 9, e0146.

Wilson, V.G. (2017) Viral Interplay with the Host Sumoylation System. Adv. Exp. Med. Biol. 963, 359-388.

Withers, J. and Dong, X. (2017) Post-translational regulation of plant immunity. Curr. Opin. Plant Biol. 38, 124-132.

Wu, L., Mao, L. and Qi, Y. (2012) Roles of DICER-LIKE and ARGONAUTE proteins in TAS-derived small interfering RNA-triggered DNA methylation. Plant Physiol. 160, 990-999.

Xie, Y., Zhao, L., Jiao, X., Jiang, T., Gong, H., Wang, B., Briddon, R.W. and Zhou, X. (2013) A recombinant begomovirus resulting from exchange of the C4 gene. J. Gen. Virol. 94, 1896-1907.

Xie, Z., Fan, B., Chen, C. and Chen, Z. (2001) An important role of an inducible RNA-dependent RNA polymerase in plant antiviral defense. Proc. Natl. Acad. Sci. 98, 6516-6521.

Xie, Z., Johansen, L.K., Gustafson, A.M., Kasschau, K.D., Lellis, A.D., Zilberman, D., Jacobsen, S.E. and Carrington, J.C. (2004) Genetic and functional diversification of small RNA pathways in plants. PLoS Biol. 2, 642-652.

Xiong, R. and Wang, A. (2013) SCE1, the SUMO-Conjugating Enzyme in Plants That Interacts with NIb, the RNA-Dependent RNA Polymerase of Turnip Mosaic Virus, Is Required for Viral Infection. J. Virol. 87, 4704-4715.

Xu, D., Lin, F., Jiang, Y., Ling, J., Hettiarachchi, C., Tellgren-Roth, C., Holm, M., Wei, N. and Deng, X.W. (2015) Arabidopsis COP1 SUPPRESSOR 2 Represses COP1 E3 Ubiquitin Ligase Activity through Their CoiledCoil Domains Association. PLoS Genet. 11, e1005747.

Xu, M., Mazur, M., Tao, X. and Kormelink, R. (2020) Cellular RNA Hubs: Friends and Foes of Plant Viruses. Mol. Plant-Microbe Interact. 33, 40-54.

Yadav, R.K. and Chattopadhyay, D. (2011) Enhanced viral intergenic region-specific short interfering rna accumulation and DNA methylation correlates with resistance against a geminivirus. Mol. PlantMicrobe Interact. 24, 1189-1197.

Yamaguchi, H., Ohnishi, J., Saito, A., Ohyama, A., Nunome, T., Miyatake, K. and Fukuoka, H. (2018) An NB- 
LRR gene, TYNBS1, is responsible for resistance mediated by the Ty-2 Begomovirus resistance locus of tomato. Theor. Appl. Genet. 131, 1345-1362.

Yan, Z., Pérez-de-castro, A., Díez, M.J., Hutton, S.F., Visser, R.G.F., Wolters, A.A., Bai, Y. and Li, J. (2018) Resistance to Tomato Yellow Leaf Curl Virus in Tomato Germplasm. Front. Plant Sci. 9, 1198.

Yang, D.L., Zhang, G., Tang, K., Li, J., Yang, L., Huang, H., Zhang, H. and Zhu, J.K. (2016) Dicer-independent RNA-directed DNA methylation in Arabidopsis. Cell Res. 26, 66-82.

Yang, J., Lin, R., Sullivan, J., Hoecker, U., Liu, B., Xu, L., Deng, X.W. and Wang, H. (2005) Light regulates COP1-mediated degradation of HFR1, a transcription factor essential for light signaling in Arabidopsis. Plant Cell 17, 804-821.

Yang, J.G., Wang, S.P., Liu, W., Li, Y., Shen, L.L., Qian, Y.M., Wang, F.L. and Du, Z.G. (2016) First report of Milk vetch dwarf virus associated with a disease of Nicotiana tabacum in China. Plant Dis. 100, 1255-1255.

Yang, L.P., Fang, Y.Y., An, C.P., Dong, L., Zhang, Z.H., Chen, H., Xie, Q. and Guo, H.S. (2013) C2-mediated decrease in DNA methylation, accumulation of siRNAs, and increase in expression for genes involved in defense pathways in plants infected with beet severe curly top virus. Plant J. 73, 910-917.

Yang, S.J., Carter, S.A., Cole, A.B., Cheng, N.H. and Nelson, R.S. (2004) A natural variant of a host RNAdependent RNA polymerase is associated with increased susceptibility to viruses by Nicotiana benthamiana. Proc. Natl. Acad. Sci. 101, 6297-6302.

Yang, X., Guo, W., Ma, X., An, Q. and Zhou, X. (2011) Molecular characterization of Tomato leaf curl China virus, infecting tomato plants in China, and functional analyses of its associated betasatellite. Appl. Environ. Microbiol. 77, 3092-3101.

Yang, X., Wang, Y., Guo, W., Xie, Y., Xie, Q., Fan, L. and Zhou, X. (2011) Characterization of small interfering RNAs derived from the Geminivirus/Betasatellite complex using deep sequencing. PLoS One 6, e16928.

Yang, X., Xie, Y., Raja, P., Li, S., Wolf, J.N., Shen, Q., Bisaro, D.M. and Zhou, X. (2011) Suppression of methylation-mediated transcriptional gene silencing by $\beta C 1$-sahh protein interaction during geminivirus-betasatellite infection. PLoS Pathog. 7, e1002329.

Yang, X., Zhou, M., Qian, Y., Xie, Y. and Zhou, X. (2014) Molecular variability and evolution of a natural population of tomato yellow leaf curl virus in Shanghai, China. J. Zhejiang Univ. Sci. B 15, 133-142.

Ye, J., Yang, J., Sun, Y., Zhao, P., Gao, S., Jung, C., Qu, J., Fang, R. and Chua, N.H. (2015) Geminivirus Activates ASYMMETRIC LEAVES 2 to Accelerate Cytoplasmic DCP2-Mediated mRNA Turnover and Weakens RNA Silencing in Arabidopsis. PLoS Pathog. 11, e1005196.

Ye, R., Chen, Z., Lian, B., et al. (2016) A Dicer-Independent Route for Biogenesis of siRNAs that Direct DNA Methylation in Arabidopsis. Mol. Cell 61, 222-235.

Yi, C. and Deng, X.W. (2005) COP1 - From plant photomorphogenesis to mammalian tumorigenesis. Trends Cell Biol. 15, 618-625.

Yong Chung, H., Lacatus, G. and Sunter, G. (2014) Geminivirus AL2 protein induces expression of, and interacts with, a calmodulin-like gene, an endogenous regulator of gene silencing. Virology 460-461, 108-118.

Yoo, B.C., Kragler, F., Varkonyi-Gasic, E., Haywood, V., Archer-Evans, S., Lee, Y.M., Lough, T.J. and Lucas, W.J. (2004) A systematic small RNA signaling system in plants. Plant Cell 16, 1979-2000.

Yu, A., Saudemont, B., Bouteiller, N., et al. (2015) Second-Site Mutagenesis of a Hypomorphic argonaute1 Allele Identifies SUPERKILLER3 as an Endogenous Suppressor of Transgene Posttranscriptional Gene Silencing. Plant Physiol. 169, 1266-1274.

Yu, D., Fan, B., MacFarlane, S.A. and Chen, Z. (2003) Analysis of the involvement of an inducible Arabidopsis RNA-dependent RNA polymerase in antiviral defense. Mol. Plant-Microbe Interact. 16, 206-216.

Zamir, D., Ekstein-Michelson, I., Zakay, Y., et al. (1994) Mapping and introgression of a tomato yellow leaf curl virus tolerance gene, TY-1. Theor. Appl. Genet. 88, 141-146.

Zeng, Y., Yi, R. and Cullen, B.R. (2003) MicroRNAs and small interfering RNAs can inhibit mRNA expression by similar mechanisms. Proc. Natl. Acad. Sci. 100, 9779-9784.

Zerbini, F.M., Briddon, R.W., Idris, A., et al. (2017) ICTV virus taxonomy profile: Geminiviridae. J. Gen. Virol. 98, 131-133.

Zhang, H., Gong, H. and Zhou, X. (2009) Molecular characterization and pathogenicity of tomato yellow leaf curl virus in China. Virus Genes 39, 249-255. 
Zhang, H., Lang, Z. and Zhu, J.K. (2018) Dynamics and function of DNA methylation in plants. Nat. Rev. Mol. Cell Biol. 19, 489-506.

Zhang, J., Dong, J., Xu, Y. and Wu, J. (2012) V2 protein encoded by Tomato yellow leaf curl China virus is an RNA silencing suppressor. Virus Res. 163, 51-58.

Zhang, X., Yuan, Y., Pei, Y., Lin, S., Tuschl, T., Patel, D.J. and Chua, N.-H. (2006) Cucumber mosiac virusencoded 2b suppressor inhibits Arabidopsis Argonaute1 cleavage activity to counter plant defense. Genes Dev. 20, 3255-3268.

Zhang, X., Zhu, Y., Liu, X., et al. (2015) Suppression of endogenous gene silencing by bidirectional cytoplasmic RNA decay in Arabidopsis. Science 348, 120-123.

Zhang, Xian, Lai, T., Zhang, P., et al. (2019) Mini review: Revisiting mobile RNA silencing in plants. Plant Sci. 278, 113-117.

Zhang, Z., Chen, H., Huang, X., et al. (2011) BSCTV C2 Attenuates the Degradation of SAMDC1 to Suppress DNA Methylation-Mediated Gene Silencing in Arabidopsis . Plant Cell 23, 273-288.

Zheng, X., Zhu, J., Kapoor, A. and Zhu, J.K. (2007) Role of Arabidopsis AGO6 in siRNA accumulation, DNA methylation and transcriptional gene silencing. EMBO J. 26, 1691-1701.

Zhou, X. (2013) Advances in Understanding Begomovirus Satellites. Annu. Rev. Phytopathol. 51, 357-381.

Zrachya, A., Glick, E., Levy, Y., Arazi, T., Citovsky, V. and Gafni, Y. (2007) Suppressor of RNA silencing encoded by Tomato yellow leaf curl virus -Israel. Virology 358, 159-165.

Zrachya, A., Kumar, P.P., Ramakrishnan, U., Levy, Y., Loyter, A., Arazi, T., Lapidot, M. and Gafni, Y. (2007) Production of siRNA targeted against TYLCV coat protein transcripts leads to silencing of its expression and resistance to the virus. Transgenic Res. 16, 385-398. 


\section{English Summary}

Tomato yellow leaf curl virus (TYLCV) is a monopartite begomovirus within the family Geminiviridae with a single stranded (ss) circular DNA genome of $2.6 \mathrm{~kb}$. TYLCV is one of the most devastating plant viruses worldwide and causes major yield losses in economically important crops like tomato. Its vector Bemisia tabaci is difficult to control and therefore breeding for resistance is the most effective way to combat TYLCV infections. In 2013, the first resistance gene against TYLCV, in casu Ty-1, was cloned and turned out to code for an RNA-dependent RNA polymerase (RDR) from the $\gamma$-class. While the members from the $\alpha$-class (RDR1, 2 and 6 , from Arabidopsis thaliana) have recognized functions in the amplification of the RNA interference (RNAi) response, either towards Post transcriptional gene silencing (PTGS) or Transcriptional gene silencing (TGS), the function of members of the $\gamma$-class (RDR3, 4 and 5) was completely unknown. Just prior to the start of this research first evidence was collected on the involvement of $T y-1$ in the enhancement of antiviral RNAi. This thesis aimed to further unravel the molecular working mechanism of Ty-1 and its applicability in light of resistance spectrum and durability.

Ty-1 also turned out to be effective against Beet curly top virus (BCTV), a member of the Curtovirus genus (Chapter 2) and very distinct from TYLCV in the genus Begomovirus, supporting the idea that $T y-1$ resistance is likely generic against all geminiviruses. Tomato $T y-1$ introgression lines as well as stably transformed tomato and Nicotiana benthamiana ( $N$. benthamiana) plants expressing the $T y-1$ gene, showed resistance against TYLCV and BCTV. A betasatellite, often found coreplicating with monopartite begomoviruses and encoding a suppressor of TGS $(\beta C 1)$, was observed to compromise Ty-1 resistance. Not only co-replication of the betasatellite with TYLCV, but also co-expression of the encoded $\beta C 1$ gene from a potato virus X (PVX) construct led to increased TYLCV titers in Ty-1 expressing plants (Chapter 2). This indicates that viruses/betasatellites encoding viral suppressors of TGS compromise $T y-1$ resistance against the geminivirus during a co-infection.

In order to find out whether Ty-1 was involved in the PTGS and/or TGS response, small RNAs were purified from TYLCV-infected susceptible Moneymaker (MM) and $T y$-1 bearing tomato plants, and sequenced to determine viral small interfering 
(vsi)RNA profiles. Compared to MM plants, profiles from Ty-1 tomato plants revealed a drastic reduction in the amount of 21-nt vsiRNAs, while the levels of those from the 22-nt and 24-nt size classes had increased. The genomic distribution of vsiRNAs also changed, showing less pronounced hotspots in Ty-1 plants (transitivity) and a relatively enhanced targeting of the Intergenic Region and the $V 1$ gene. Whereas the increase in 24-nt vsiRNAs clearly suggests an enhanced TGS response, the elevated level of 22-nt vsiRNAs was unexpected and intriguing. Whether these 22-nt vsiRNAs play a role in a PTGS response or guide targeting of viral DNA for TGS via a noncanonical RdDM pathway, remains to be determined (Chapter 3).

Cytoplasmic Processing bodies (P-bodies) are responsible for RNA decay of defective RNA molecules and they compete with neighbouring siRNA-RDR6 (PTGSamplification) bodies for these aberrant RNA molecules as substrate. Whereas impairing cytoplasmic P-bodies led to an enhanced PTGS response against a transgene (GFP), it did not change the susceptibility against TYLCV (Chapter 4). P-bodies thus do not seem to compete for RNA substrates with RDRs involved in amplifying the RNAi response against TYLCV and this finding suggests that the latter probably takes place somewhere else.

In Chapter 5, Ty-1 was in situ localized in plant cells by transiently expressing GFPtagged Ty-1 in N. benthamiana. The Ty-1 protein localized in nuclear bodies (NBs), in close proximity to COP1- containing photobodies. In the additional presence of TYLCV, the number of Ty-1 NBs increased and Ty-1 translocated to Cajal bodies. In addition, bimolecular fluorescence complementation assays showed interactions between Ty-1 and components of the sumoylation machinery, namely SIZ1, SCE1, SUMO1, and SUMO3. The interplay with the sumoylation machinery also appeared influenced by TYLCV infection. Since Cajal bodies were previously shown to host many components of the TGS pathway, these findings altogether point towards the involvement of Ty-1 in a TGS response.

In conclusion, evidence is presented of the applicability of $T y-1$ to a wider range of geminiviruses, but at the same time an Achilles heel became visible, namely co-infections with other viruses that suppress TGS may break Ty-1 resistance. Plants containing Ty-1 show increased levels of 24-nt vsiRNAs, needed for a TGS 
response, and of 22-nt vsiRNAs, of which the role in TGS or PTGS still needs to be determined. RDRs localized in cytoplasmic siRNA bodies and required for PTGS amplification, do not appear to be involved in an anti-geminiviral RNAi response. This fits with Ty-1 being localized in the nucleus, where the protein shows a dynamic distribution profile depending on the absence or presence of TYLCV infection, and interplays with the sumoylation machinery. In Chapter 6, the practical and scientific implications of the data presented in this thesis are discussed and summarized in a model to present a first glimpse of the mode of action of Ty-1 in a TGS response against geminiviruses. 


\section{Nederlandse samenvatting}

Tomato yellow leaf curl virus (TYLCV) is een begomovirus binnen de Geminiviridae familie en bevat één enkelstrengs circulair DNA molecuul van $2.6 \mathrm{~kb}$ als genoom. TYLCV is één van de meest verwoestende plantenvirussen ter wereld en leidt tot grote verliezen in economisch belangrijke gewassen zoals tomaat. De vector Bemisia tabaci (de tabakswittevlieg) is moeilijk onder controle te houden en daarom is het kweken van resistente tomatenplanten de effectiefste manier om TYLCV infecties te bestrijden. In 2013 werd het eerste resistentie gen tegen TYLCV gekloneerd, genaamd $T y$-1, en dit gen bleek te coderen voor een RNA-afhankelijkRNA-polymerase (RNA-dependent-RNA-polymerase, RDR) van de $\gamma$-klasse. Leden van de $\alpha$-klasse (RDR1, 2 en 6 in Arabidopsis thaliana) hebben erkende functies in de amplificatie van RNA interferentie (RNAi, ook wel RNA-silencing genoemd) met een rol in transcriptional-gene-silencing (TGS) of post-transcriptional-genesilencing (PTGS). Voor RDRs van de $\gamma$-klasse (RDR3, 4 en 5) was de functie tot dan toe volkomen onbekend. Net voor de start van dit onderzoek werd het eerste bewijs geleverd voor de betrokkenheid van $T y-1$ in de versterking van antivirale RNAi. Het doel van dit proefschrift was om het moleculaire werkingsmechanisme van $T y-1$ verder te bestuderen en de toepasbaarheid van dit resistentiegen te onderzoeken met het oog op de duurzaamheid van de resistentie en mogelijke werking tegen andere virussen dan TYLCV.

Ty-1 bleek ook effectief te werken tegen Beet curly top virus (BCTV), een lid van het Curtovirus genus in de Geminiviridae familie (Hoofdstuk 2) en duidelijk verschillend van het begomovirus TYLCV. Deze bevinding ondersteunde het idee dat $T y$-1 resistentie waarschijnlijk generiek is tegen alle geminivirussen. Zowel $T y-1$ introgressielijnen van tomaat als stabiel getransformeerde tomaten en Nicotiana benthamiana ( $N$. benthamiana) planten die het $T y-1$ gen tot expressie brengen, vertoonden resistentie tegen zowel TYLCV als BCTV. Daarnaast bleek een betasatelliet, een in de natuur veelvuldig voorkomend en co-replicerend DNA molecuul van TYLCV en andere geminivirussen, de $T y-1$ resistentie te ondermijnen. Deze beta-satelliet codeert voor $\beta C 1$, een remmer van TGS. Niet alleen co-replicatie van de beta-satelliet met TYLCV, maar ook co-expressie van slechts het $\beta C 1$ gen vanaf een potato-virus-X (PVX)-construct, leidde tot verhoogde TYLCV titers in Ty-1 
planten (Hoofdstuk 2). Met andere woorden, waar virussen normaal in staat zijn om antivirale RNAi te onderdrukken door te coderen voor virale remmers van RNAi, blijken diegene die de TGS reactie remmen (óf vanaf een beta-satelliet óf vanaf een ander virus tijdens een co-infectie) de Ty-1 resistentie tegen geminivirussen te ondermijnen dan wel te verzwakken.

Om erachter te komen of Ty-1 betrokken was bij de TGS en/of de PTGS reactie, werden kleine RNA moleculen in de grootteorde van $<50$ nucleotiden (nt) gezuiverd uit TYLCV-geïnfecteerde, vatbare Moneymaker (MM) planten of uit Ty-1 coderende, resistente tomatenplanten. Van deze RNA moleculen werd vervolgens de nucleotidesequentie bepaald om de virale, kleine interfererende RNA (viral-smallinterfering, vsiRNA) profielen te bepalen. In vergelijking met MM planten lieten de profielen van $T y-1$ tomatenplanten een drastische reductie zien in de hoeveelheid 21-nt vsiRNAs, terwijl die van 22-nt en 24-nt in aantal toenamen. De distributie van vsiRNAs over het virale genoom was ook anders en vertoonde minder uitgesproken hotspots in Ty-1 planten en relatief meer vsiRNAs gericht tegen de intergene regio en het virale $V 1$ gen. Terwijl de toename van 24-nt vsiRNAs duidelijk verwees naar een versterkte TGS respons, waren de verhoogde niveaus van 22-nt vsiRNAs onverwacht en intrigerend. Of deze een rol spelen in een PTGS reactie of leiden tot een TGS reactie tegen viraal DNA via een onbekende RNA-afhankelijke-DNAmethylering (RdDM)-route, moet nog worden opgehelderd (Hoofdstuk 3).

Cytoplasmatische "Processing-bodies" (P-bodies) zijn verantwoordelijk voor het afbreken van RNA wat niet functioneel is, bijv door de aanwezigheid van een prematuur stop codon. P-bodies concurreren met naburige siRNA-RDR6 (PTGSamplificatie) "bodies" voor deze afwijkende RNA moleculen. Terwijl het verstoren van cytoplasmatische P-bodies leidde tot een verhoogde PTGS reactie tegen een GFP transgen, veranderde dit niet de vatbaarheid voor TYLCV (Hoofdstuk 4). P-bodies lijken dus niet te concurreren voor RNA substraat met RDRs die betrokken zijn bij het versterken van de RNAi reactie tegen TYLCV, en dit suggereert dat deze respons waarschijnlijk ergens anders plaatsvindt.

In Hoofdstuk 5 werd Ty-1 in situ gelokaliseerd in plantencellen door GFP-gelabeld Ty-1 tot expressie te brengen in $N$. benthamiana. Ty- 1 lokaliseerde in nucleaire 
structuren (Nuclear-bodies, NBs) in de nabijheid van zogenaamde "photobodies" waarin het eiwit COP1 aanwezig is. In de aanwezigheid van TYLCV, nam het aantal Ty-1 bevattende NBs toe en verplaatste Ty-1 zich ook naar Cajal-bodies. Bovendien lieten bimoleculaire fluorescentie complementatie (BIFC) analyses interacties zien tussen Ty-1 en componenten van de sumoylerings machinerie, namelijk SIZ1, SCE1, SUMO1 en SUMO3. De interactie met de sumoylerings machinerie bleek ook beïnvloed te worden door TYLCV infectie. Aangezien eerder al was aangetoond dat componenten van de TGS-reactie zich (onder andere) in Cajal-bodies bevinden, wijzen al deze bevindingen samen op de betrokkenheid van Ty-1 in een TGS respons.

Concluderend levert dit proefschrift bewijs voor de toepasbaarheid van Ty-1 tegen een breder scala aan geminivirussen, maar het laat ook de achilleshiel zien, namelijk co-replicerende virussen of beta-satellieten die coderen voor remmers van TGS. Planten die coderen voor Ty-1 vertonen verhoogde niveaus van 24-nt vsiRNAs, nodig voor een TGS respons, en van 22-nt vsiRNA's, waarvan de rol in TGS of PTGS nog moet worden vastgesteld. RDR's, gelokaliseerd in de cytoplasmatische siRNA-bodies en vereist voor PTGS-amplificatie, lijken niet betrokken te zijn bij een (sterke) anti-geminivirale RNAi. Dit past bij de bevinding dat Ty-1 gelokaliseerd is in de kern, waar het eiwit, afhankelijk van de aan- of afwezigheid van TYLCV, een dynamisch distributieprofiel vertoont, en een wisselwerking heeft met de sumoylatie machinerie. Ontrafeling van de rol van sumoylatie bij de werking van Ty-1 zal een van de uitdagingen voor de toekomst zijn. In Hoofdstuk 6 worden de praktische en wetenschappelijke implicaties van alle bevindingen die in dit proefschrift worden gepresenteerd, besproken en samengevat in een model om een eerste blik te werpen op het werkingsmechanisme van Ty-1 in een TGS-respons tegen geminivirussen. 


\section{Acknowledgements/Dankwoord}

En dan is het einde van dit PhD avontuur bereikt! Het was niet altijd makkelijk, maar toch heb ik er nooit spijt van gehad dat ik hieraan begonnen ben. Dankzij alles wat ik heb geleerd op zowel onderzoeks als persoonlijk vlak, maar vooral dankzij alle mensen die ik deze jaren heb mogen ontmoeten en waarmee ik heb mogen samenwerken. Je PhD project is dan wel "jouw project", maar een PhD doe je zeker niet alleen. Daarom ben ik heel blij dat ik op deze plek iedereen kan bedanken die dit boekje mede mogelijk heeft gemaakt. Mocht ik iemand vergeten zijn te bedanken, dan bij deze ook jij bedankt!

Allereerst wil ik mijn promotie-team bedanken: Richard, Monique, en Yuling. Richard, als mijn dagelijkse begeleider hebben we veel wetenschappelijke discussies, meetings, koffie- en lunch-pauzes samen gehad. Bedankt dat je vertrouwen in mij had en mij, met mijn niet-planten achtergrond, deze kans op een PhD gegeven hebt. Ik vond het heel fijn dat je deur altijd open stond voor vragen en adviezen over experimentele set-ups, inclusief ALLE nodige controles. Jouw input tijdens het schrijfproces en altijd zeer snelle feedback op manuscripten hebben me veel geholpen. Ik ben blij dat je mijn Schelluins-kwartiertje (een paar minuten te laat binnen komen rennen) hebt gedoogd zonder veel commentaar. Jouw enthousiasme voor de wetenschap en grote kennis binnen de plantenvirologie zijn een grote inspiratie. Daarnaast hebben we veel lol gehad tijdens de plant-viro sociale activiteiten in Amersfoort, zoals de pool party en met $z$ ' $n$ allen schaatsen. Ook dank aan je vrouw die ons altijd heel hartelijk heeft ontvangen. Monique, bedankt voor de gezellige praatjes tijdens de pauzes, wetenschappelijke discussies, voor je input op mijn scriptie en het vertrouwen dat ik het goed zou afronden. Je snelle reacties op mijn manuscripten waren zeker in de laatste fase erg nuttig. Yuling, I would like to thank you for the provision of plant material, the inspiring joint meetings and your input on manuscripts. I hope the collaboration between Virology and Plant Breeding will continue to be fruitful in the future.

I would like to thank all the plant scientists with whom I collaborated in the project. Myluska, you were working on geminiviruses when I started my PhD and I'm very thankful that you got me familiar with the work in the greenhouse and taught me 
how to perform geminivirus infections. Later on, Yan became my plant-breedingproject-buddy: our joint experiments, which resulted in the broad-spectrum resistance paper, were always very "gezellig" and it was an honour to work with you. Magda, the localization chapter would not have been the same without your help! Thanks for the discussions on sumoylation and the help at the confocal microscope. Beside the lab-work, I'm happy you were around as a friend: you gave perfect distractions of lab life during drinks, dinners, game-nights and our holiday to China as a special highlight!

Ook dank aan alle andere plantenvirologen voor de gezellige sfeer in het lab, het gevoel dat iedereen zichzelf kan zijn en als zodanig gewaardeerd wordt. Dick Lohuis, van jouw heb ik geleerd hoe leuk het is om te spelen met protocollen en dingen die niet werken toch werkend te krijgen. Ik ben blij dat ik, nog net vooraf aan je pensioen, met je heb kunnen samenwerken. Maria and Cristina, you took over the job as plant virology technicians. I only worked briefly with you in the lab, but it was nice to have you around and I wish you all the best! Min, thanks for all the nice chats in the office and the lab, and good luck with finishing up your PhD, you are almost there! Mandy, ik ben blij dat met jouw komst het werk aan gemini's doorgaat. Je bent een geweldige opvolger voor dit project en ik kijk nu al uit naar je boekje!

Then two plant virologist that need an extra special word: Irene and André, my plant buddies. My PhD would not have been the same without you two and I am honoured that you want to join me in this special moment and will be my paranymphs. Irene, heel veel dank voor je luisterend oor. Ik kon altijd bij jou terecht om even te klagen, maar ook voor een lach en een koffie/thee. Je eerlijke mening heb ik altijd erg gewaardeerd. Ook op creatief/DIY vlak (van zelf gebreide kerstballen en zelfgemaakte zonneschermen tot props voor de one-shot-productions) heb je altijd inspiratie. Jouw input in de one-shot-productions heeft de filmpjes zeker naar een heel nieuw niveau gebracht. Ik vind het heel fijn dat je mijn paranymph wilt zijn, terwijl je ook heel druk bent met het laatste stukje van je eigen PhD traject. Heel veel succes met de laatste loodjes en ik weet zeker dat je harde werken gaat resulteren in een mooi boekje. Ik wens jou en Lotte heel veel geluk en gezondheid! André, since the moment you entered the lab and turned out to be my neighbour ("roomy"), we had a lot of fun! Thanks for accepting me as I am, with you I can make 
every horrible joke that pops up in my head without holding back. Thanks for all the nice drinks and dances, but also the good conversations and scientific input and help in the lab. My Portuguese is still far from fluent, but with your help combined with DuoLingo, at least I won't accidentally say inappropriate things in Portuguese. I also would like to thank your partner Karol: I am very happy you moved to Wageningen and that I got the opportunity to get to know you (and doggie Lis).

During these years I had the honour to be paranymph for several PhD defences and this has resulted in special bonds. Athos, your dark humour and sometimes slightly pessimistic views balanced out with my enthusiasm. Only you, me and Dick worked in the plant lab when I started. Your scientific help at that time was very valuable to me, next to of course the social activities outside the lab. I hope to meet you again in the future on conferences or maybe on a trip to Brasil. Giel, met jou heb ik zo veel lol gehad op virologie: zingen en dansen op feestjes, maar zeker ook in het lab! Ik vind het nog steeds jammer dat we door de verhuizing van lab en kantoor verder van elkaar kwamen te zitten, maar gelukkig waren er genoeg sociale momenten buiten werk: BBQs, diners, een labtrip organiseren, salsa lessen, een road trip door Nederland, enzovoorts. Je was mijn "social partner in crime"! Ook onze wetenschappelijke discussies waren altijd interessant en je hulp bij de small RNA data-analyse heeft me ontzettend geholpen. Ik ben blij dat jij nu je plekje hebt gevonden bij MSD! Han, you were very good company during the late evenings working in the lab and office. Your mean jokes are really not that bad, I actually miss your humour a lot! I think back with much joy to my first day at Virology when we got lost during the labtrip, and I very much appreciated the ice cream you got me in Bennekom to compensate. The fish we adopted when you moved to the UK are still alive, so you still remain around a bit in Wageningen. I hope you and Chunyue are doing well back in China and hope to see you again in the future!

Verder wil ik graag alle andere mensen bedanken die virologie maken tot de geweldige plek die het is. De vaste medewerkers Vera, Jan, Just, Gorben, Rene, Jeroen, jullie deur stond altijd open voor een praatje en de spontane wetenschappelijke discussies bij de koffiemachine hebben me meer dan eens op weg geholpen. Jan, ik heb genoten van onze grappige discussies en BBQs bij jou thuis. En excuses dat je me tijdens (en soms buiten) koffiepauzes in je kantoor kon 
horen. De analisten Corinne, Els, Marleen, zonder jullie zou het lab niet zijn wat het is. Jullie structuur is een fijne houvast tijdens het soms chaotische PhD leven. Bedankt voor het helpen met technische problemen en het uit de brand helpen als er plotseling iets op was! Hanke ook jou ben ik veel dank verschuldigd, ook al kan ik het je niet meer persoonlijk vertellen. Marleen en Melanie bedankt voor alle hulp met de administratieve kant van het PhD traject. Also, thanks to all other (current and previous) colleagues in the department. Leo (and Brenda), you left Wageningen some time ago but your Brazilian socialness is something I will never forget. Amaya, Aydin, Bob, Fengqiao, Gabriela, Haidong, Irene Meki, Jelke, Jerome, Jitte, John, Linda, Marcel, Marcio, Mariana, Mehtap, Mia, Miao, Sandra, Simone, Tessy, Qiushi thanks for all the fun at international dinners, lab trips, coffee and lunch breaks, (a lot of!) pies, participation in one shot productions and the random chats. Many new people arrived after I left the office, from who I only got to know a few, Sharella, Gwen, Joyce, and Emilyn. I hope you will have an equal fun time at virology as I had!

Ook wil ik alle virologie collega's van PRI (Wageningen Research/Prime Diagnostics) bedanken. Het was fijn om met jullie een grotere plantenvirologie-groep te vormen en expertises te kunnen delen. In het bijzonder Jan B en Annette, bedankt voor de gezellige praatjes, wetenschappelijke discussies en hulp in het lab (Jan, het spelen met de Octet was een mooi project). Petra bedankt voor virus stocks die we via jullie konden gebruiken.

Buiten ons eigen lab heb ik ook andere apparatuur kunnen gebruiken en technische support gehad voor qPCRs en de confocale microscoop van Marga en Norbert. Bedankt voor jullie expertise en hulp.

Ik heb heel veel planten gebruikt tijdens experimenten in de kas en klimaatkamer. Mannen van Unifarm Bert, Bertus en Henk, heel erg bedankt voor de leuke praatjes in de kas en alle goede zorgen voor onze plantjes. Het was heel fijn dat mijn lastminute wijzigingen (toch meer zaailingen, toch ook een paar bentha's erbij) altijd mogelijk waren. Jullie werk was essentieel voor het slagen van de experimenten.

During my project we started to have "dominant resistance gene meetings" with colleagues from nematology and phytopathology, which made collaborations 
between the departments easier. Thanks to Aska, Matthieu, Aranka (special thanks for helping with co-IPs), Yinbin, Octavina and Erik for making these meetings possible and useful.

With a lot of joy, I have supervised several BSc and MSc students during my PhD. Dominique, Fabian, Gijs, Jingyi, Joep, Marijke, Nils, and Reindert, I would like to thank you all for the useful contribution to the project and the fun you brought to the lab and greenhouse! Mariangela, you started when I was writing and you were supervised by Magda. It was nice to get to know you and thanks a lot for your hard work, which resulted in data included in chapter 5 .

Afgelopen zomer tijdens de lockdown had ik moeite om vooruit te komen met mijn scriptie en de Summer writing weeks, georganiseerd door het Writing Lab, hebben me weer goed op weg geholpen. Ik wil daarom Joke hartelijk bedanken voor deze schrijf-weken. Ook Susan van EPS bedankt voor de ondersteuning in die tijd.

During these years in Wageningen I also made good friends outside the uni. First of all, the Wageningen Salsa people: The LaFrique family and the dancers of Lucia and Santos. I started dancing salsa with LaFrique in 2016 and up to today, I am still happy with that decision! It turned out to be the best way to take my mind off work. Via salsa I got to know some good friends: Luigi, Nicola, Mara, Eva and Uros. Thank you all for the salsa classes, Sunday Salsas, chats, dances, and more. I also would like to thank Amalia, Laura, Leah and Jaume. I am always happy to meet up with you for dinner, picknicks, coffee, a stroll over the market, having a laugh or a good conversation. Thanks for providing a lot of fun in free time.

Iskra, we are living together already for 5 years! I remember reading your "roomapplication" letter and being doubtful about your rats. However, I am so happy that we picked you as new roomy. We can talk about anything and you really feel like family. My PhD would have been so much harder without you. You dragged me out of the house when I was working too much, listened to me complaining or being excited about my science, we talked about life, watched movies, went to concerts and parties, went on holiday, jointly woke up late with coffee, and much more. Thanks for being here! I am very happy you met Rik and you are having 
a good time together in Ghana. I wish you all the luck in the world! Rik, thanks for the fun dinners and game nights, you are good company with your dry word jokes and amazing Wordfeud skills. Tamara, een paar dagen nadat jij bij ons introk ging het land in lockdown: een test die we zeker hebben doorstaan. We hebben elkaar leren kennen in een hele stressvolle periode en ik ben blij dat ik altijd even kon spuien en daarna weer opgelucht door kon. Bedankt voor de gezelligheid, lekkere cappuccino's, mij voederen met gezond eten tijdens de laatste maand voor de deadline, goeie gesprekken, grapjes, en dansjes tijdens thuis concerten. Ik wil ook graag ex-huisgenootjes Debby en Anna bedanken. Debby, jouw gedrevenheid en (sportieve) energie waren inspirerend. Ik wens jou en Jamie heel veel geluk en hoop dat jullie bruiloft dit jaar groots door kan gaan! Anna, I am happy you are still around in Wageningen and it is always nice to catch up with you during coffee/ tea/dinner. The writing together in the office the last month of our PhDs was perfect. Ik wil ook mijn collega's van het WICC bedanken, vooral Robin, Tsizyana, Alex, Erwin en Petertje, waarmee ik de meeste diensten draaide. Bedankt voor de gezellige diensten die een goeie afwisseling vormden voor het schrijven thuis.

When I moved to Wageningen I mostly thought of it as a small, bit boring village (sorry!). However, with all of you around it turned out the opposite: a lively city with always something fun or interesting activity going on!

Verder wil ik graag mijn vrienden van "voor de PhD" bedanken. Thanks to the I\&l'ers Anna and Danyta. I am happy we stayed in touch, despite our busy lives and living far away. Our catch-up skypes during the last year were very relaxing and I hope we can meet up in real life again soon. Daniëlle, Elke en Mirjam, ofwel de BMW'ers: Ook al is er veel veranderd in de 13 jaar dat we elkaar kennen (van eerstejaars BSc student tot allemaal Dr!) en wonen we verspreid door Nederland, als we afspreken is het altijd gewoon goed. Laten we onze gezellige high-teas, etentjes en andere uitjes er zeker inhouden! Daniëlle, Nijmegen is gelukkig vrij dichtbij en onze extra uitjes en thuiswerkdagen waren een perfecte afleiding voor het schrijven en de lockdown. Annemarie en Nick, wij gaan helemaal "way back" tot de Oudehoven. We zien elkaar niet vaak, maar ook met jullie heb ik altijd het gevoel alsof er niets is veranderd en we verder gaan waar we gebleven waren. Bedankt daarvoor! 
De Kruisjes, Ik heb er even over nagedacht waar jullie het beste in dit dankwoord pasten en uiteindelijk net voor de familie. Want ook al zijn we geen familie, het voelt wel een beetje zo. Jullie deur staat altijd open om even langs te wippen als ik in Schelluinen ben, lammetjes te knuffelen en bij te praten. Frans, jouw nuchtere vragen over mijn onderzoek zijn heel relativerend "Wanneer heeft de boer hier nog iets aan?". Ik vind het vreselijk dat Frans Jelle er niet meer is en wens jullie heel veel sterkte.

En dan wil ik graag afsluiten met mijn familie. Onze familie is klein, maar ook heel hecht en ik kan onmogelijk op papier uitdrukken hoe blij ik ben dat jullie er zijn. Ome Piet en Frank, ik wil jullie bedanken voor de gezellige verjaardagen, koffies, spelletjes, de altijd goede klusadviezen en hulp. Het is nog steeds onwerkelijk dat tante Atie niet meer in ons midden is en ik ben trots hoe jullie hier samen je weg in hebben gevonden. Lieve Oma, mijn naamgenootje, ik ben heel blij dat jij mijn promotie mee kan maken. Mijn wereldje op de uni is heel anders dan de jouwe en soms is het lastig uit te leggen wat ik nu precies voor werk doe, maar toch vraag je hoe het nou met dat boekje is en ben je geïnteresseerd. Je interesse en vertrouwen in mij betekenen veel voor me. Het is jammer dat opa er niet meer is. Mijn liefde voor planten is met hem begonnen, met ons vaste rondje door de moestuin en boomgaard, en ik weet zeker dat hij mijn onderzoek naar tomaten reuze interessant had gevonden. Peter en Jolijn, bedankt dat jullie altijd voor me klaar staan als er iets is. Jolijn, jij begeeft je ook in het academische wereldje en het is fijn om met jou te kunnen sparren over universitaire perikelen. Peter, ik zou willen dat ik wat meer van jouw nuchtere kijk op deze wereld had. Je maakt je niet snel druk en kan daardoor ook mij vaak geruststellen en dingen relativeren. Ik zou me geen betere broer en schoonzus kunnen wensen. Pa en ma, ik kan absoluut niet in woorden vatten hoeveel jullie voor me betekenen. Bedankt dat ik altijd alles bij jullie kwijt kan en dat jullie me door dik en dun steunen. Bij jullie thuis zijn, geeft rust in mijn hoofd en daarna kan ik weer met nieuwe moed (en ouwe hekel) verder. Zonder jullie was dit boekje er niet geweest. We zien het vaak als vanzelfsprekend, maar het is bijzonder en mooi dat wij het altijd zo fijn hebben met $z$ ' $n$ allen. Ik ben jullie ontzettend dankbaar voor dit warme nest en ik houd heel veel van jullie. 


\section{About the author}

Cornelia (Corien) Maaike Voorburg was born the 3th of June 1989 in Gorinchem and was raised in the nearby village of Schelluinen. She went to primary school at the Stoutjesdijkschool in Schelluinen and afterwards attended the Gymnasium at the Lyceum Oudehoven in Gorinchem. In 2007 Corien started studying Biomedical Sciences at Utrecht University. Cell biology and virology became her favourite courses and therefore the bachelor study was followed by the master Infection \& Immunity at the same university. The continuous "battle" between pathogens and the defence system intrigued

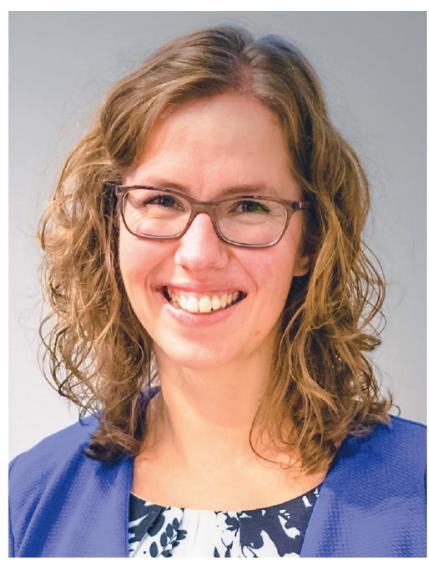
her. During a 9-month internship at the Department of Medical Microbiology of Utrecht University under supervision of Dr Robert Jan Lebbink and Prof. Emmanuel Wiertz, she identified host genes involved in virus-mediated downregulation of MHC class I molecules in immune cells. Subsequently, Corien moved to London to perform a 6-month internship at the Department of Respiratory Medicine of Imperial College London under supervision of Dr Lydia Durant and Prof. Peter Openshaw. Here, Corien investigated the roles for regulatory T cells (Tregs) in Respiratory Syncytial Virus (RSV) infection. Her work during these internships contributed to publications in Nature Communications and the Journal of Virology. After her graduation as Master of Science, she pursued a PhD position to continue studying the interactions between hosts and viruses. She switched fields from mammalian viruses to plant viruses when starting her PhD studies in the Laboratory of Virology at Wageningen University under supervision of Dr Richard Kormelink, Prof. Yuling Bai and Prof. Dr Monique van Oers. During her PhD studies she investigated the molecular mechanism behind the resistance gene $T y-1$ and the effect of viral encoded RNA silencing suppressors on this resistance. Corien was also enthusiastically involved in education: she guided students during projects of the courses "Cell Biology and Health" and "Molecular Aspects of Bio-interactions", she assisted during the practicals of "Molecular Virology" and was the daily supervisor of $1 \mathrm{BSc}$ and $7 \mathrm{MSc}$ thesis students. Her curiosity towards the interactions between hosts and pathogens remains a driving force for Corien and she wishes to pursue a further career in this field. 


\section{List of publications}

Voorburg CM, Bai Y, Kormelink R. Small RNA profiling of susceptible and resistant Ty-1 encoding tomato plants upon TYLCV infection. (in preparation)

Voorburg CM, Mazur MJ, Ravensbergen M, D’aniello M, Lohuis D, Kormelink R. The Ty-1 encoded RNA-dependent RNA polymerase from the gamma class, conferring resistance to geminiviruses, localizes to nuclear bodies involved in RNAi response and sumoylation. (in preparation)

Voorburg CM, Yan Z, Bergua-Vidal M, Wolters AA, Bai Y, Kormelink R. 2020. Ty-1, a universal resistance gene against geminiviruses that is compromised by co-replication of a betasatellite. Molecular Plant Pathology 21: 160-172.

Van De Weijer ML, Bassik MC, Luteijn RD, Voorburg CM, Lohuis MAM, Kremmer E, Hoeben RC, Leproust EM, Chen S, Hoelen H, et al. 2014. A high-coverage shrna screen identifies TMEM129 as an E3 ligase involved in ER-associated protein degradation. Nature Communications 5: 3832.

Durant LR, Makris S, Voorburg CM, Loebbermann J, Johansson C, Openshaw PJM. 2013. Regulatory T Cells Prevent Th2 Immune Responses and Pulmonary Eosinophilia during Respiratory Syncytial Virus Infection in Mice. Journal of Virology 87: 1094610954. 


\section{Education Statement of the Graduate School Experimental Plant Sciences \\ Issued to: \\ Date: \\ Group: \\ University: \\ C.M. Voorburg \\ 18 June 2021 \\ Laboratory of Virology \\ Wageningen University \& Research}

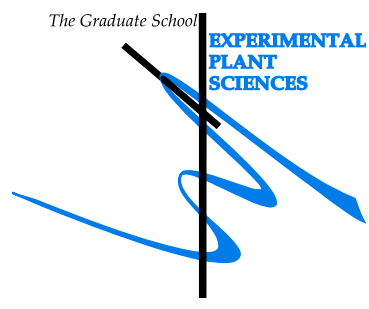

date

RNAi against geminiviruses

Writing or rewriting a project proposal

Writing a review or book chapter

MSc courses

Subtotal Start-Up Phase

1,5

2) Scientific Exposure

date

\section{EPS PhD student days}

Get2Gether for EPS PhD students, Soest, NL

29-30 Jan 2015

Get2Gether for EPS PhD students, Soest, NL

28-29 Jan 2016

Get2Gether for EPS PhD students, Soest, NL

9-10 Feb 2017

Get2Gether for EPS PhD students, Soest, NL

$15-16$ Feb 2018

EPS theme symposia

EPS Theme 2 Symposium \& Willie Commelin Scholten Day 2015, Utrecht, NL

20 Feb 2015

EPS Theme 2 Symposium \& Willie Commelin Scholten Day 2016, Leiden, NL

$22 \operatorname{Jan} 2016$

EPS Theme 2 Symposium \& Willie Commelin Scholten Day 2017, Wageningen, NL

23 Jan 2017

EPS Theme 2 Symposium \& Willie Commelin Scholten Day 2018, Amsterdam, NL

$24 \operatorname{Jan} 2018$

EPS Theme 2 Symposium \& Willie Commelin Scholten Day 2019, Wageningen, NL

1 Feb 2019

Lunteren Days and other national platforms

Annual meeting Experimental Plant Sciences, Lunteren, NL

13-14 Apr 2015

Annual meeting Experimental Plant Sciences, Lunteren, NL

11-12 Apr 2016

Annual meeting Experimental Plant Sciences, Lunteren, NL

10-11 Apr 2017

Annual meeting Experimental Plant Sciences, Lunteren, NL

9-10 Apr 2018

Dutch Annual Virology Symposium (DAVS) 2015, Amsterdam, NL

6 Mar 2015

Dutch Annual Virology Symposium (DAVS) 2016, Amsterdam, NL

11 Mar 2016

Dutch Annual Virology Symposium (DAVS) 2017, Amsterdam, NL

3 Mar 2017

Dutch Annual Virology Symposium (DAVS) 2018, Amsterdam, NL

9 Mar 2018

Seminars (series), workshops and symposia

Seminars:

Dick de Ridder and Paul Kersey, Plant Sciences Seminar on Bioinformatics

Marilyn Roossinck, "Plant Virus Ecology and Evolution: Pathogens to Mutualists", Rob Goldbach Virology Lecture 
Claude Fauquet, "The application of plant biotechnology for preventing diseases", Plant Sciences Seminar

EPS flying seminar (George Coupland) "Seasonal flowering in annual and perennial plants"

4 Dec 2014

Kevin Foster, "The evolution of cooperation and competition in microbes", WEES seminar

19 Jan 2015

Renato Resende, "Tospoviruses in Brazil: From basic to applied research"

22 Jan 2015

Julien Varaldi, "Behaviour manipulation and horizontal gene transfer in a virus-parasitoid

27 Jan 2015

interaction", WEES seminar

Eduardo Bejarano, "Geminiviruses: masters at redirecting and reprogramming plant processes"

22 Oct 2015

Michael Diamond, "New insights into Zika Virus Pathogenesis, Rob Goldbach Virology Lecture

13 Sep 2016

Mart Krupovic, "Natural history of viral capsids", WEES seminar

17 Nov 2016

23 Feb 2017

Martin Cann, "The immune receptor Rx1 remodels chromatin and chromatin interactors in

immunity", EPS Flying Seminar

Andrea Gröne, "Why bother? Disease is normal in wildlife", WEES seminar

11 Jul 2017

Peter Simmonds, "RNA virus genomes: more than just a coding sequence?"

28 Sep 2017

20 Nov 2017

Anne-Nathalie Volkoff, "Endogenous viruses used by parasitic wasps to deliver virulence molecules to their hosts", WEES seminar

Israel Pagán, "Extreme tactics of plant virus transmission: from seed dispersion to parasitic

castration", WEES seminar

14 Dec 2017

5 Apr 2018

\section{Workshops:}

transPLANT Workshop 2014 "Exploiting and understanding solanaceous genomes", Wageningen, NL

13-14 Oct 2014

Workshop "Pathogen-informed Crop Improvement", Wageningen, NL

8-10 Apr 2015

\section{Symposia:}

Symposium Antiviral defence mechanisms, Wageningen (NVBMB), NL

8 Oct 2014

Dutch Symposium on Emerging Bunyaviruses, Dronten, NL

20 Nov 2014

\section{Seminar plus}

Discussion with Eduardo Bejarano

13 Sep 2016

Master class Michael Diamond

WEES workshop with Dr. Mart Krupovic

18 Nov 2016

23 Feb 2017

\section{International symposia and congresses}

7th European Plant Science Retreat (EPSR), Paris, France

10-13 Jul 2015

5th Conference of the International Working Group on Legume and Vegetable Viruses (IWGLLV),

Haarlem, NL

8th European Plant Science Retreat (EPSR), Barcelona, Spain

31 Aug-3 Sep

2015

20-23 Jun 2016

5th International Symposium on Tomato Diseases, Malaga, Spain

13-16 Jun 2016

Sixth Joint Meeting of the DPG working Group "Virus Diseases of Plants" and the "Nederlandse Kring voor Plantenvirologie", Bonn, Germany

10th European Plant Science Retreat (EPSR), Utrecht, NL

27-28 Mar 2017

European Congress of Virology (ECV), Rotterdam, NL

3-6 Jul 2018

28 Apr - 1 May

2019

\section{Presentations}

Poster "Ty-1: triggers an epigenetic antiviral defence", EPSR Paris 


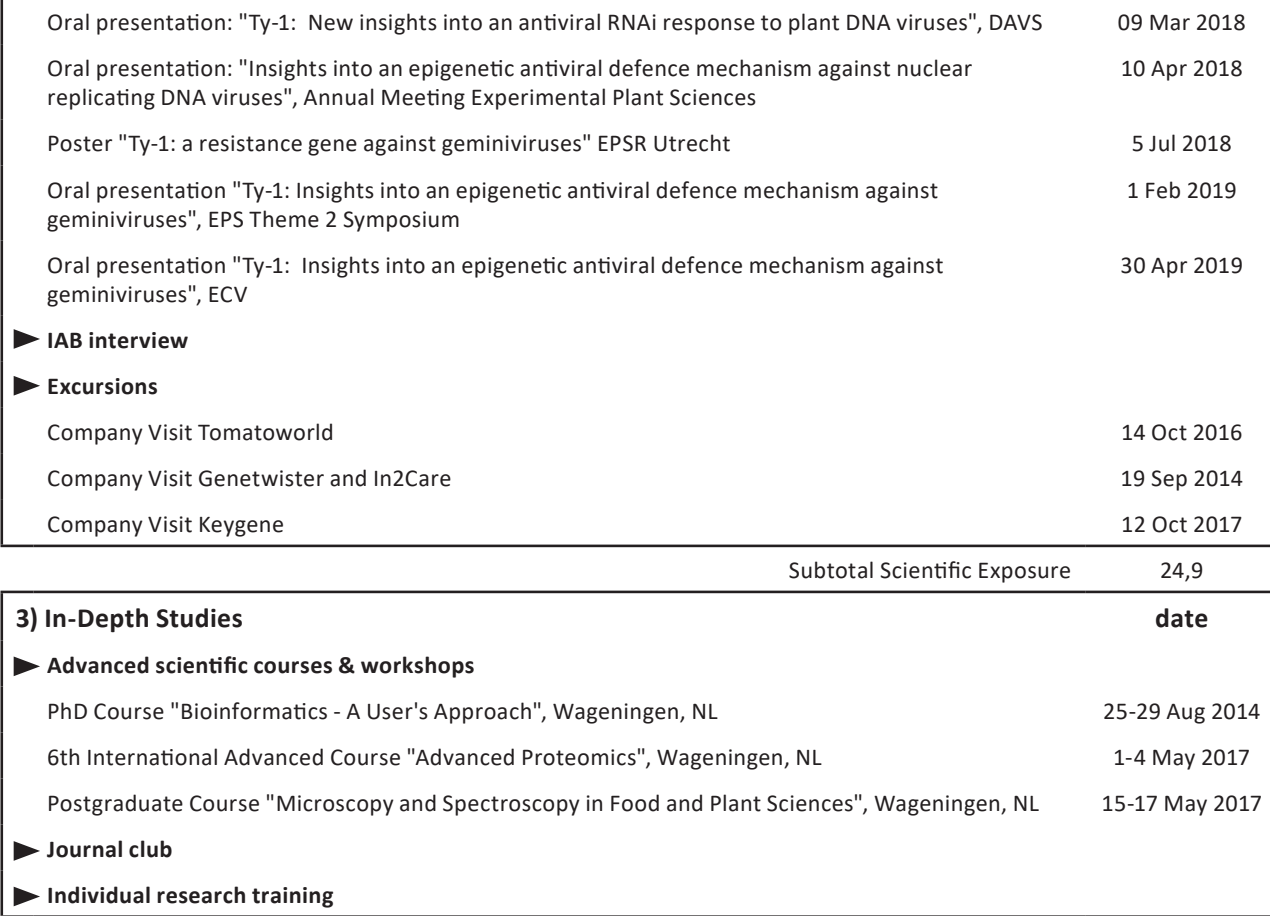

Subtotal Scientific Exposure

24,9

\section{3) In-Depth Studies}

\section{Advanced scientific courses \& workshops}

PhD Course "Bioinformatics - A User's Approach", Wageningen, NL

6th International Advanced Course "Advanced Proteomics", Wageningen, NL

\section{Journal club}

Individual research training

Subtotal In-Depth Studies

3,9

\section{4) Personal Development}

date

\section{General skill training courses}

Competence assessment, Wageningen, NL

EPS introduction course, Wageningen, NL

Adobe InDesign Essential Training, Wageningen, NL

Teaching and Supervising Thesis Students, Wageningen, NL

Scientific writing, Wageningen, NL

Career assessment, Wageningen, NL

Organisation of meetings, $\mathrm{PhD}$ courses or outreach activities

Membership of EPS PhD Council

Herewith the Graduate School declares that the PhD candidate has complied with the educational requirements set by the Educational Committee of EPS with a minimum total of 30 ECTS credits.

* A credit represents a normative study load of 28 hours of study. 
The research described in this thesis was carried out at the Laboratory of Virology at Wageningen University, The Netherlands and was financially supported by NWO-CNPq within the Joint Research Project Biobased Economy (729.004.011).

Cover design by Iliana Boshoven-Gkini, Agilecolor.com Printed by GVO drukkers \& vormgevers, Ede, The Netherlands 


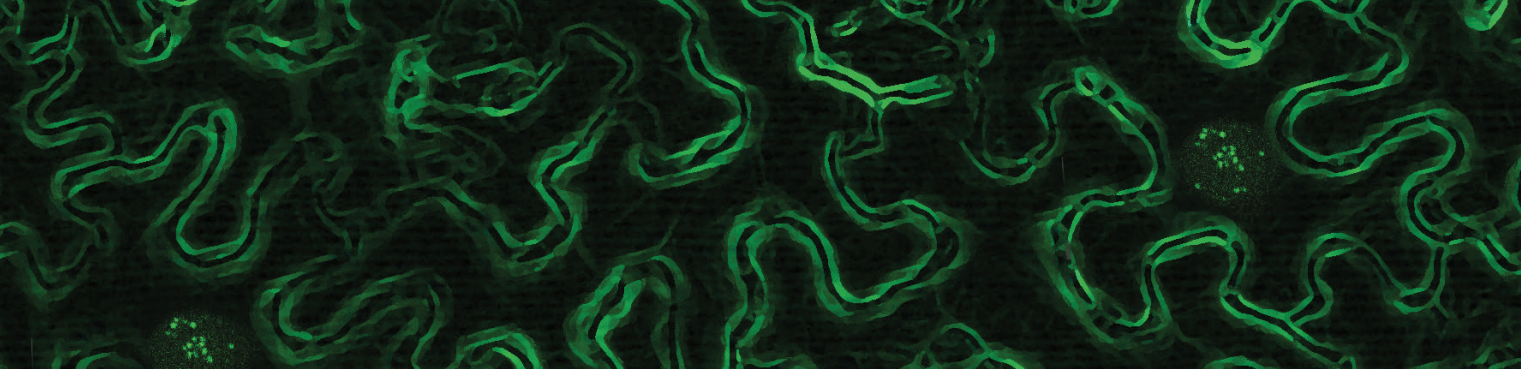

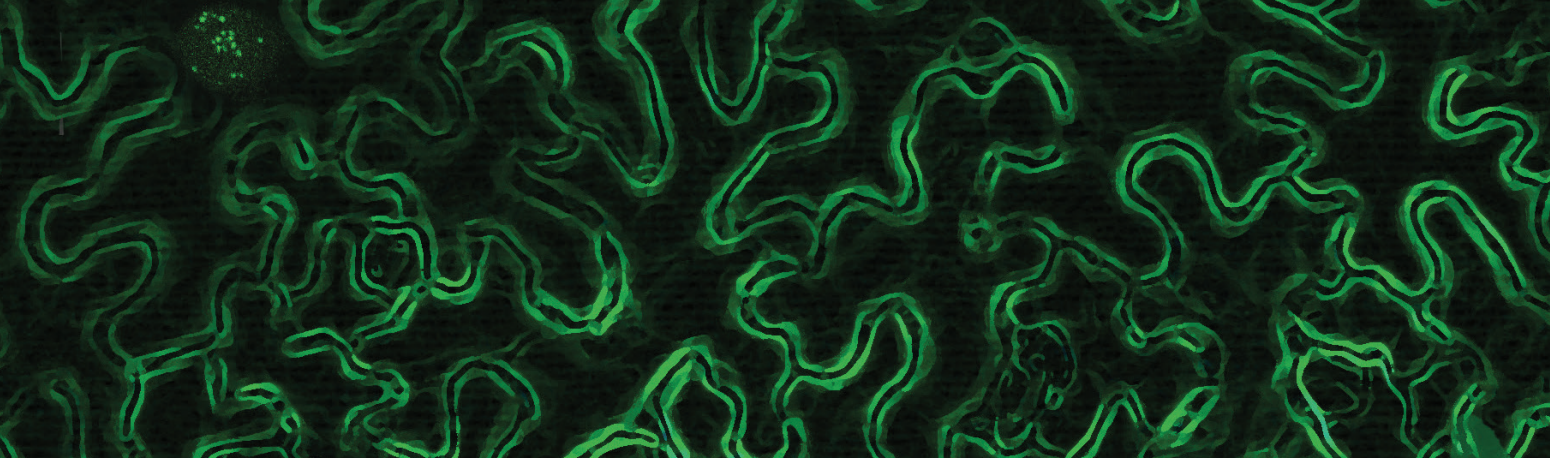
3(

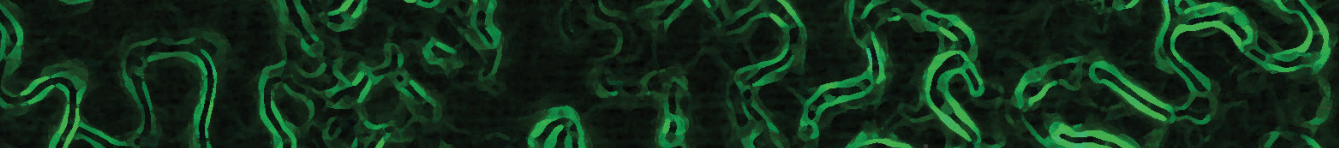

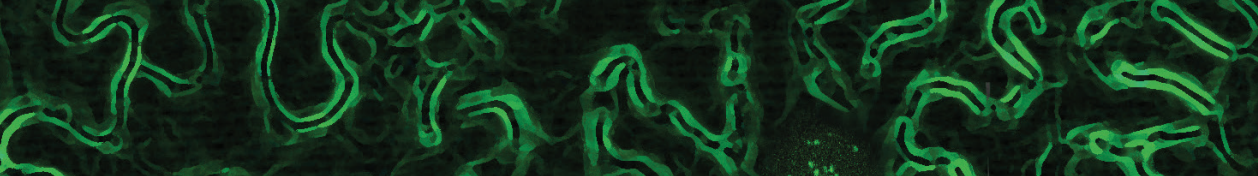

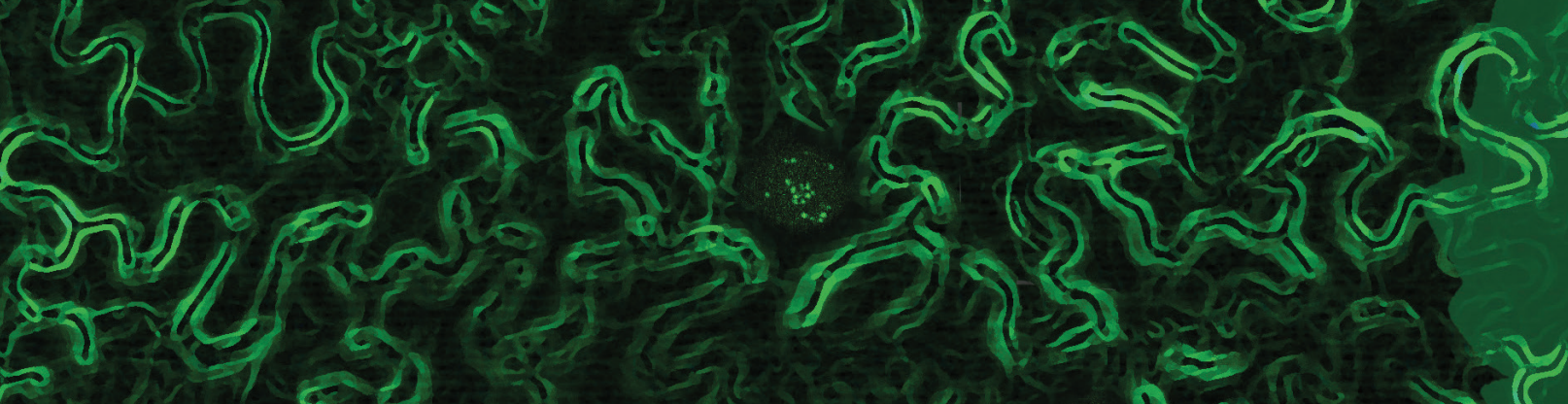
(2) 2 है

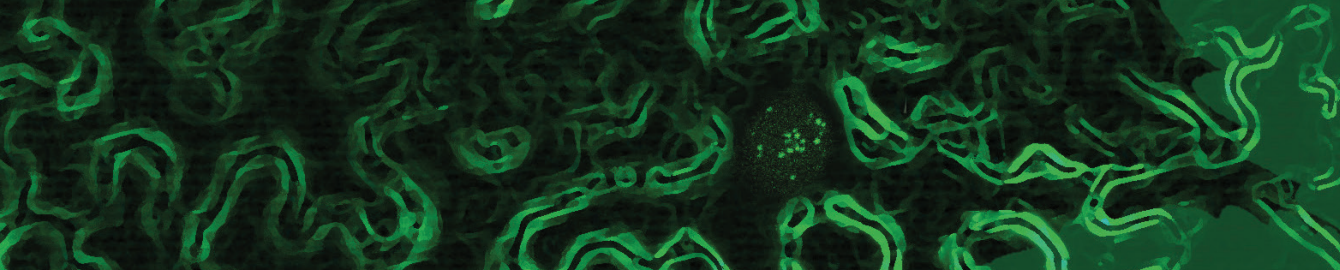
$\int 55$ an? 5

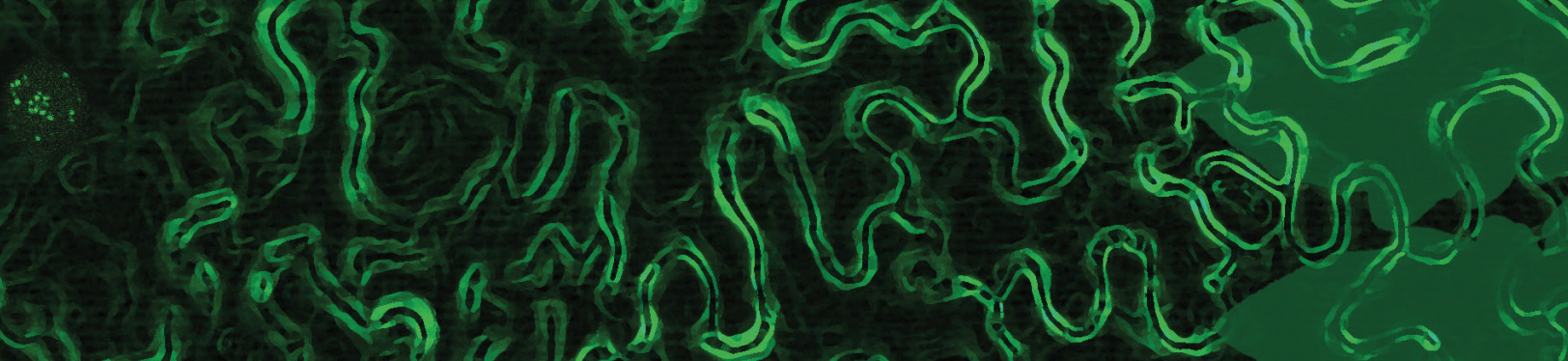

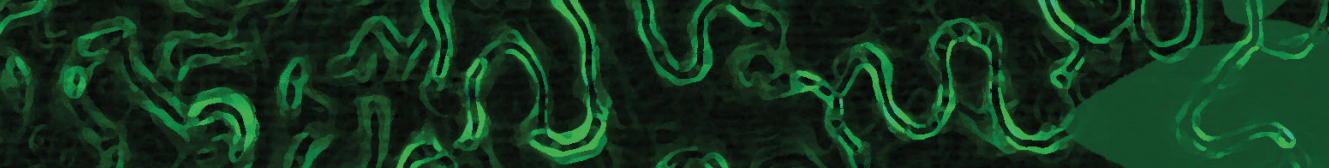
.
5)

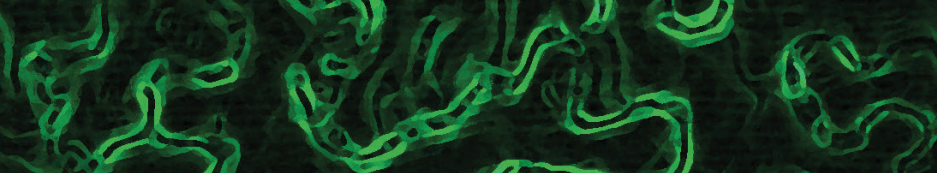 .

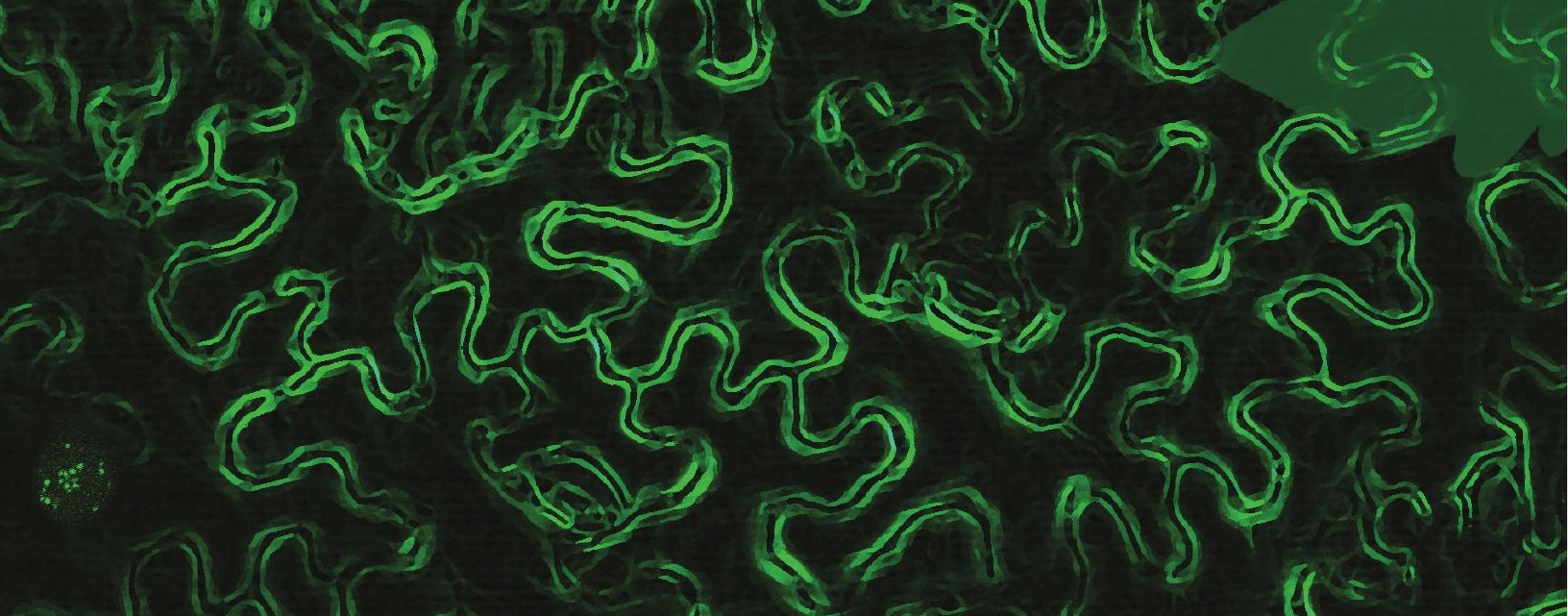

